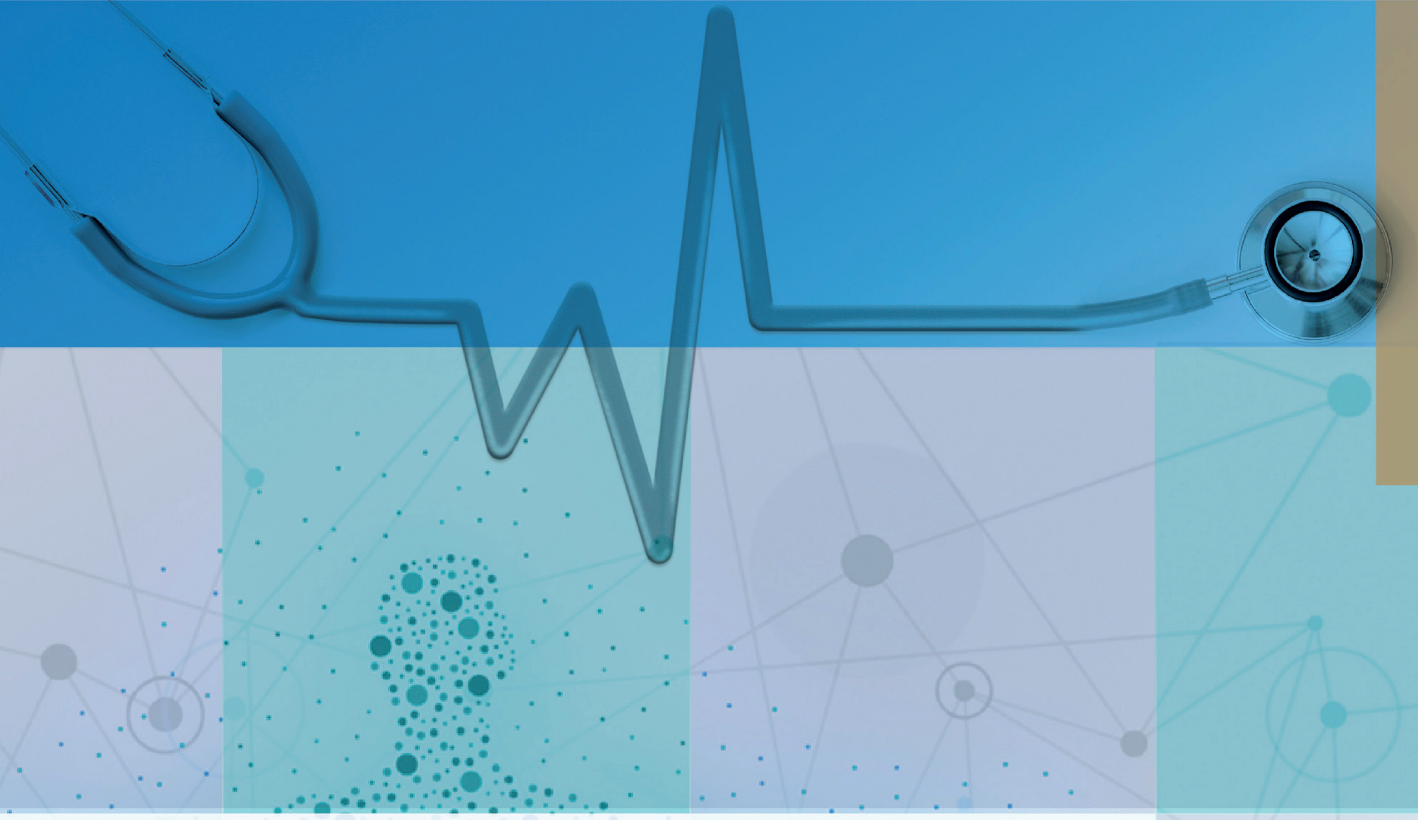

\title{
CÁlCULOS $Y$ ESCALAS PRONÓSTICAS EN MEDICINA INTERNA
}

Autores:

Héctor Fabián Ortega Castillo | Bolitigui Coulibaly | Francisco Villacorta Johanna Elizabeth Villacrés Cervantes. I Jessica Marcela Monar Carrera María José Bahamonde Gaibor | Francis Valeria Pazos Raza 



\section{UNIVERSIDAD NACIONAL DE CHIMBORAZO}

Rector

Ph.D. Gonzalo Nicolay Samaniego Erazo

\section{Vicerrectora Académica}

Ph.D. Ángela Calderón Tobar

Vicerrector de Investigación, Vinculación y Posgrado

Ph.D. Lexinton Cepeda Astudillo

\section{Vicerrectora Administrativa}

Ph.D. Anita Ríos Rivera

\section{Comité Editorial:}

Presidente: Ph.D. Lexinton Cepeda Astudillo;

Secretaria: Ing. Sandra Zúñiga Donoso.

Miembros: Ph.D. Margarita Pomboza Floril; Ph.D. Gerardo Nieves Loja; Ph.D. Carmen Varguillas Carmona; Ph.D. Cristhy Jiménez Granizo; Ph.D. Víctor Julio García; Msc. Clara Mayorga Mazón; Ph.D. Diego Pinilla Rodríguez.

Título de la obra: Cálculos y escalas Pronósticas en Medicina Interna

Nombre del autor (es): Héctor Fabián Ortega Castillo • Bolitigui Coulibaly • Francisco Villacorta • Johanna Elizabeth Villacrés Cervantes. • Jessica Marcela Monar Carrera

- María José Bahamonde Gaibor • Francis Valeria Pazos Raza ; Riobamba, Abril 2020

(C) Héctor Ortega C. MD, MPH, PhD(c), 2020

Ediciones: Universidad Nacional de Chimborazo (UNACH)

Diseño Gráfico: UNACH

Primera edición - junio 2020

Riobamba - Ecuador

Se prohíbe la reproducción total o parcial de esta obra, sea cual fuere el medio, sin la anuencia por escrito del titular de los derechos.

ISBN: 978-9942-835-10-9

ISBN: 978-9942-835-09-3 (DIGITAL)

DOI: https: / / doi.org/10.37135/u.editorial.05.16

Registro Biblioteca Nacional

Depósito legal: 058560

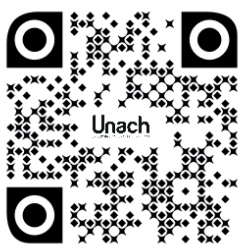




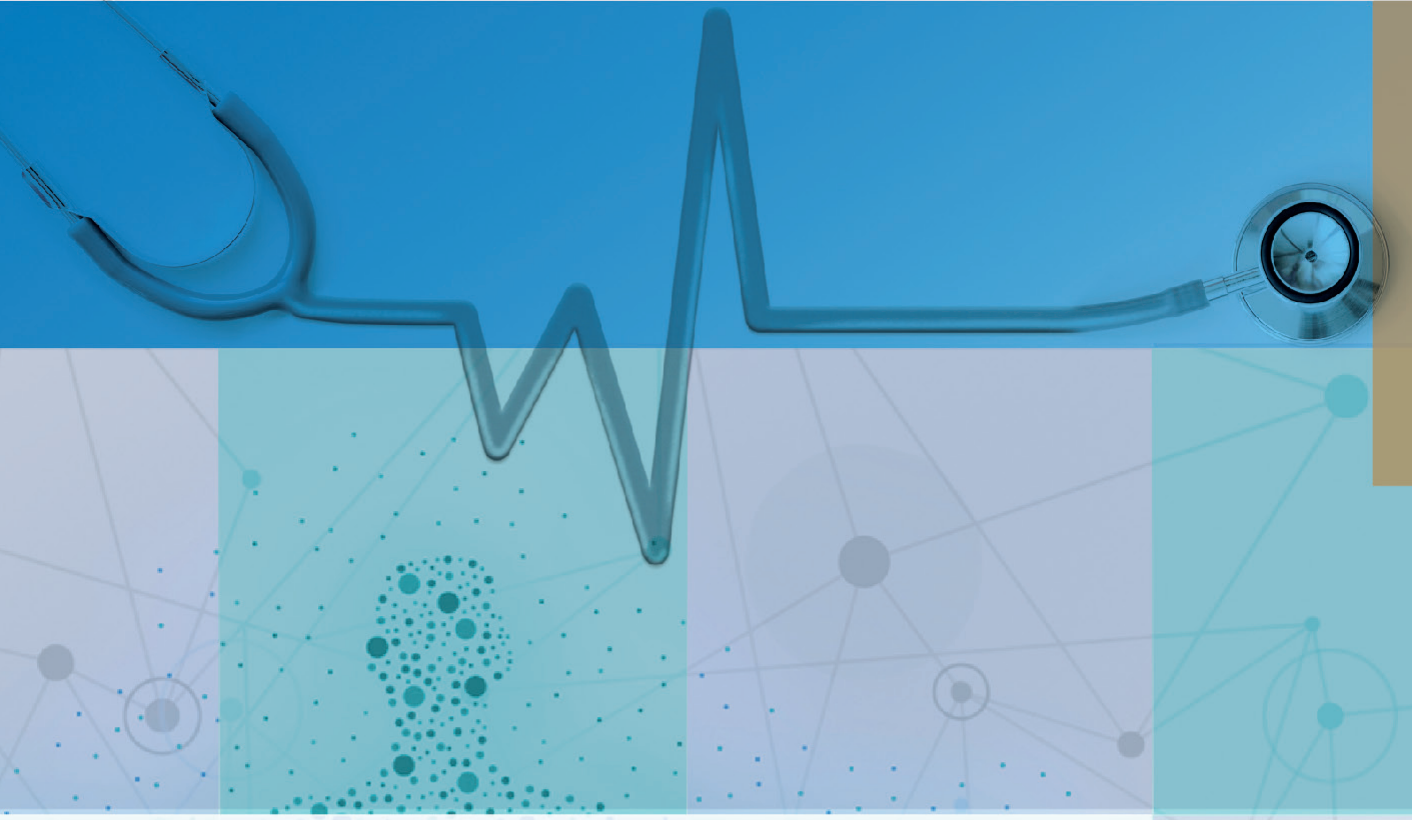

\section{CÁlCULOS $Y$ ESCALAS PRONÓSTICAS EN MEDICINA INTERNA}

Autores:

Héctor Fabián Ortega Castillo | Bolitigui Coulibaly | Francisco Villacorta Johanna Elizabeth Villacrés Cervantes. | Jessica Marcela Monar Carrera María Jose Bahamonde Gaibor | Francis Valeria Pazos Raza

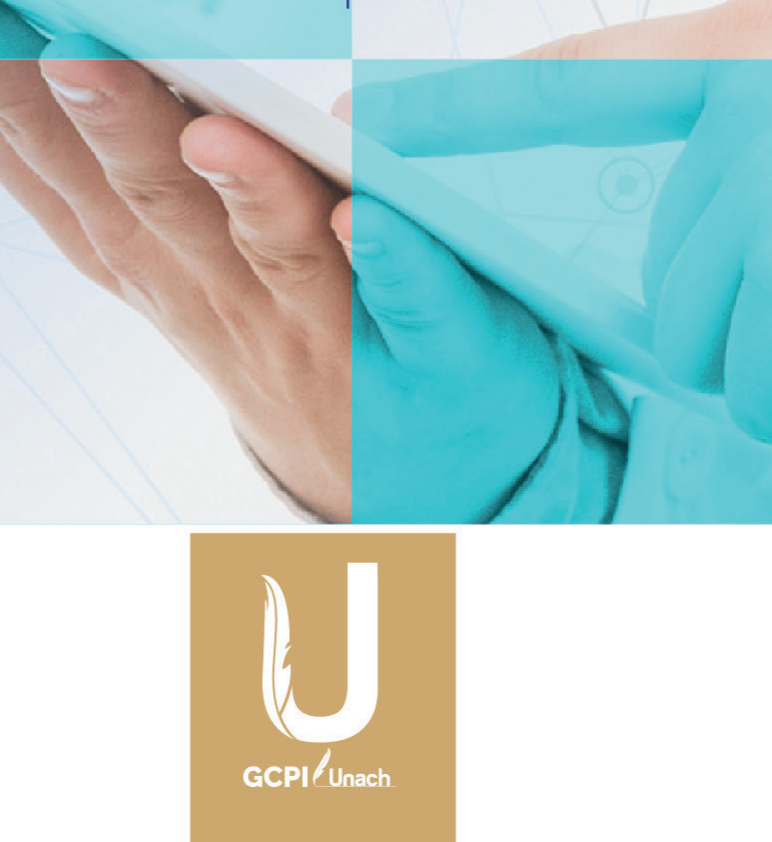




\section{ÍNDICE}

\section{NEUROLOGÍA}

ENFERMEDAD CEREBROVASCULAR HEMORRÁGICA

ESCALA DE COMA DE GLASGOW ..

ESCALA ATRIA PARA RIESGO DE HEMORRAGIA

ESCALA CINCINNATI PARA ACCIDENTE

CEREBROVASCULAR.

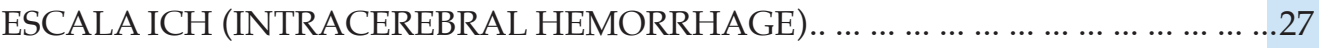

ESCALA FUNC PARA PRONÓSTICO

DE INDEPENDENCIA FUNCIONAL DESPUÉS

DE UNA HEMORRAGIA INTRACEREBRAL .

ESCALA DE HUNT \& HESS PARA CLASIFICACIÓN

DE LA HEMORRAGIA SUBARACNOIDEA

ESCALA DE RANKIN MODIFICADA PARA

DISCAPACIDAD NEUROLÓGICA

CRISIS CONVULSIVAS

ESCALA PARA ESTIMAR LA SEVERIDAD

DEL ESTATUS EPILÉPTICO

ENFERMEDAD CEREBROVASCULAR ISQUÉMICA $\ldots \ldots \ldots \ldots \ldots \ldots \ldots \ldots$

ESCALA NIHSS PARA ACV AGUDO $\ldots \ldots \ldots \ldots \ldots \ldots \ldots n$

ESCALA ABCD² PARA RIESGO

DE ACCIDENTE CEREBROVASCULAR ISQUÉMICO

POSTERIOR A UN ACCIDENTE ISQUÉMICO TRANSITORIO

CARDIOLOGÍA

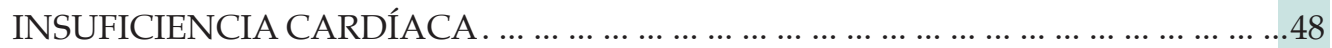

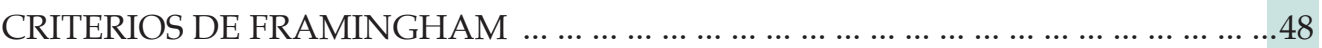

CLASIFICACIÓN FUNCIONAL DE LA INSUFICIENCIA

CARDÍACA SEGÚN LA NYHA.. 
ESTADIFICACIÓN ACC / AHA PARA LA

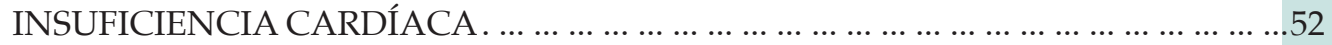

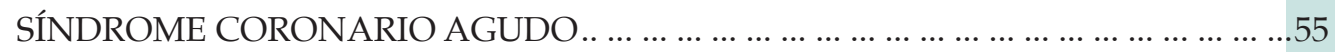

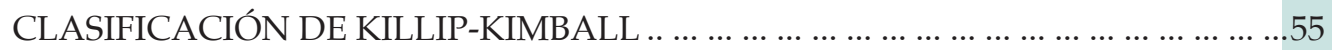

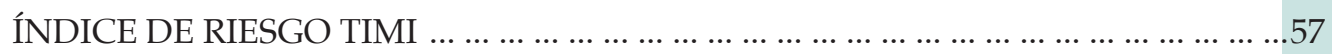

PUNTAJE DE RIESGO TIMI PARA STEMI $\ldots \ldots \ldots \ldots \ldots \ldots \ldots \ldots \ldots \ldots \ldots \ldots \ldots \ldots \ldots \ldots$

PUNTAJE DE RIESGO TIMI PARA

ANGINA INESTABLE E INFARTO DE MIOCARDIO

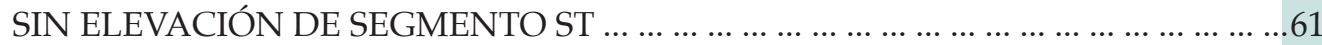

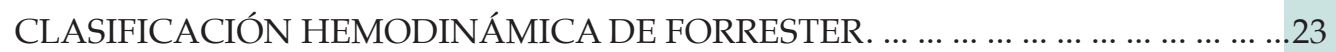

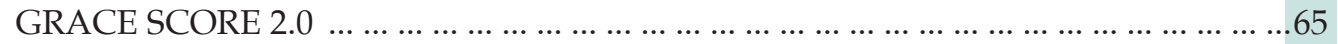

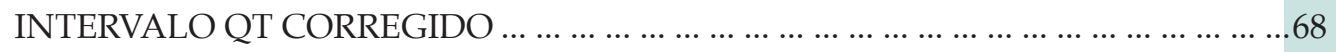

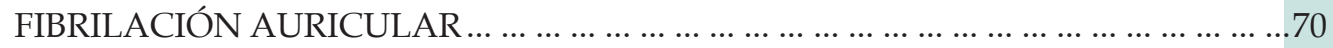

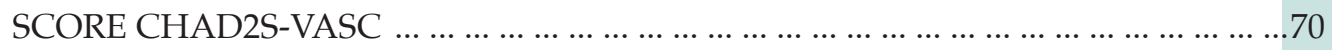

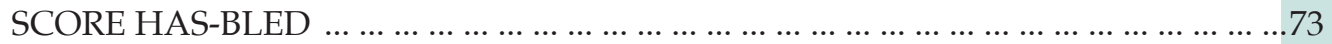

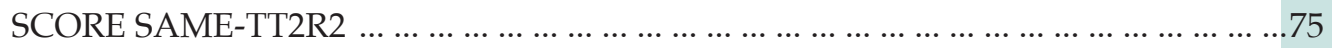

\section{NEUMOLOGÍA}

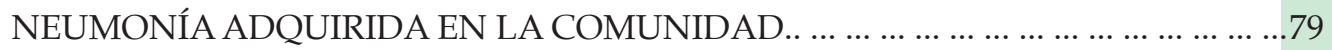

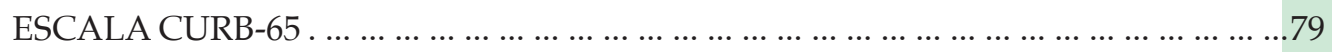

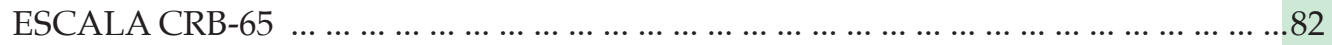

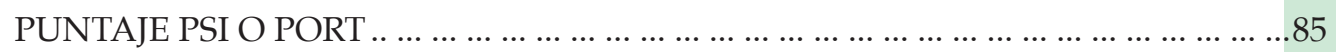

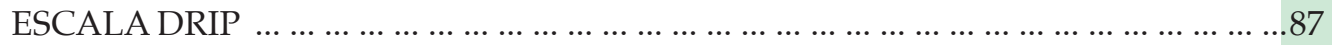

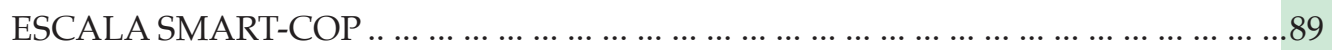

CRITERIOS DE ALIBERTI PARA NEUMONÍA

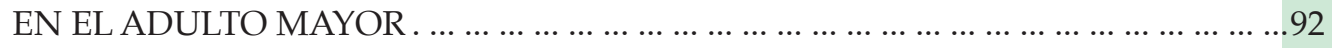


SCORE SHORR PARA RIESGO DE SARM

EN PACIENTES CON NEUMONÍA..

SCORE DE INFECCIÓN PULMONAR CLÍNICA (CPIS)

PARA LA NEUMONÍA ASOCIADA

A VENTILACIÓN MECÁNICA (VAP)..

INFLUENZA A H1N1

SISTEMA DE PUNTAJE DEL HOSPITAL UNIVERSITARIO

WINTHROP-DIVISIÓN DE ENFERMEDADES INFECCIOSAS,

PARA EL DIAGNÓSTICO DE INFLUENZA

SEVERA EN ADULTOS (MODIFICADO)..

EPOC EXACERBADO...... .101

ESCALA DE DISNEA (MMRC) MODIFICADA .101

ESCALA BAP-65 PARA LA EXACERBACIÓN AGUDA DE LA EPOC .104

ÍNDICE BODE .107

ESCALA CAT PARA EVALUACIÓN DE LA EPOC. 110

ESCALA DECAF PARA LA EXACERBACIÓN

AGUDA DE LA EPOC...

CRITERIOS GOLD (GLOBAL INITIATIVE

FOR OBSTRUCTIVE LUNG DISEASE) PARA EPOC

CRISIS ASMÁTICA

EVALUACIÓN DE LAS CRISIS DE ASMA EN ADULTO

(ALERTA 2-2010)

CÁLCULO DEL GRADIENTE ALVEOLO-ARTERIAL DE O2 $\ldots \ldots \ldots \ldots \ldots \ldots \ldots \ldots . \ldots \ldots$

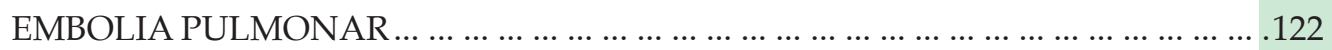

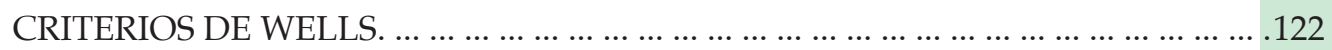

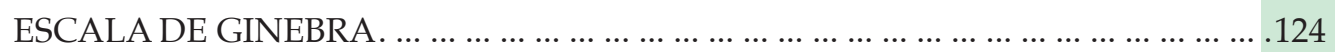

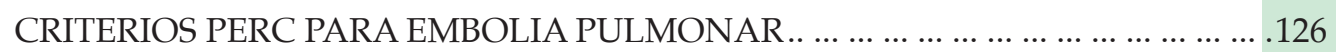


CRITERIOS DE WELLS PARA

TROMBOSIS VENOSA PROFUNDA.

FIBROSIS PULMONAR

SCORE DU BOIS PARA MORTALIDAD

POR FIBROSIS PULMONAR IDIOPÁTICA.

DERRAME PLEURAL

CRITERIOS LIGHT PARA DERRAMES EXUDATIVOS.

INTUBACIÓN ENDOTRAQUEAL.

CLASIFICACIÓN MODIFICADA DE MALLAMPATI

\section{NEFROLOGÍA}

OSMOLARIDAD SÉRICA

ANIÓN GAP EN SUERO.

DÉFICIT DE BICARBONATO.

FRACCIÓN DE EXCRECIÓN DE SODIO (FENA)

FRACCIÓN DE EXCRECIÓN DE UREA (FEUREA)

ENFERMEDAD RENAL CRÓNICA

ECUACIÓN DE CKD-EPI PARA

TASA DE FILTRACIÓN GLOMERULAR .

ECUACIÓN DE MDRD GFR

ACLARAMIENTO DE CREATININA - ECUACIÓN

DE COCKCROFT-GAULT

CALCULADORA DE RIESGO DE INSUFICIENCIA RENAL

FALLA RENAL AGUDA 
CRITERIOS RIFLE

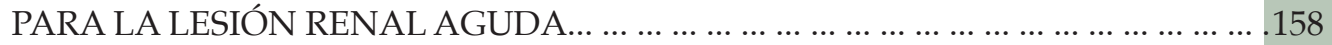

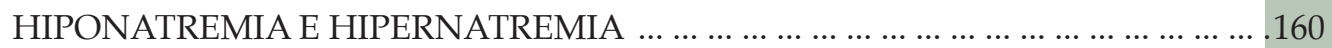

CORRECCIÓN DE SODIO POR HIPONATREMIA

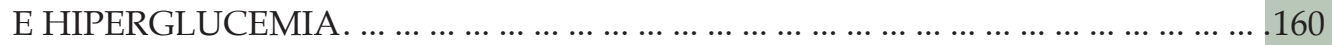

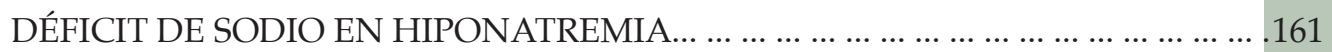

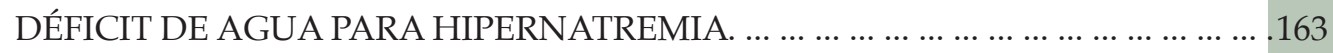

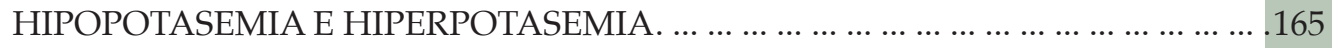

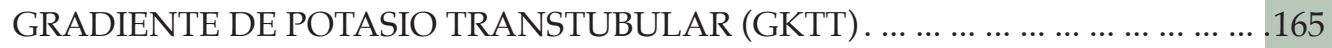

ENDOCRINOLOGÍA

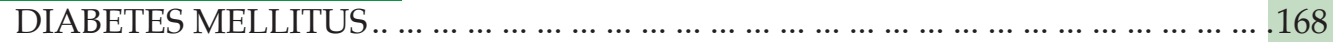

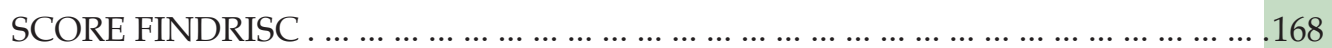

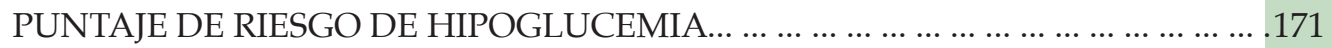

LDL CALCULADO - FÓRMULA DE FRIEDEWALD $\ldots \ldots \ldots \ldots \ldots \ldots \ldots \ldots \ldots \ldots \ldots \ldots \ldots$

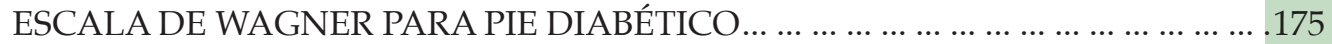

PUNTUACIÓN PEDIS

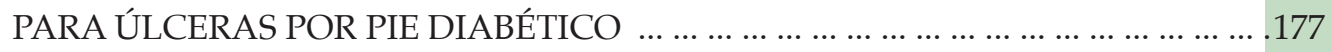

CLASIFICACIÓN IDSA PARA SEVERIDAD

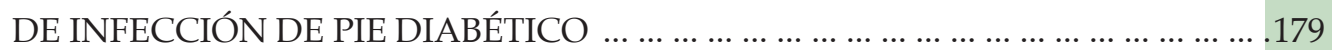

CRISIS TIROTÓXICA $\ldots \ldots \ldots \ldots \ldots$

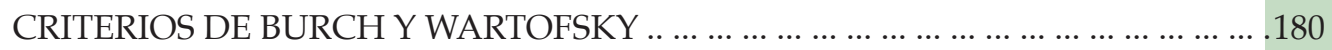

REUMATOLOGÍA

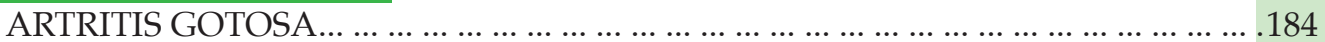

CRITERIOS DE CLASIFICACIÓN DE GOTA ACR / EULAR ............................ 184

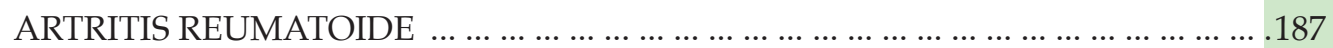

CRITERIOS DE CLASIFICACIÓN

DE LA ARTRITIS REUMATOIDE ACR / EULAR 2010 .............................. 187 
INDICE DE ACTIVIDAD DE LA ENFERMEDAD

CLÍNICA (CDAI) PARA ARTRITIS REUMATOIDE.

DAS28-PCR (PUNTUACIÓN DE ACTIVIDAD

DE LA ENFERMEDAD-28 PARA LA

ARTRITIS REUMATOIDE CON PCR)

DAS28-VSG (PUNTUACIÓN

DE ACTIVIDAD DE LA ENFERMEDAD-28 PARA

LA ARTRITIS REUMATOIDE CON VSG) .

SDAI (ÍNDICE DE ACTIVIDAD DE LA ENFERMEDAD)..

LUPUS ERITEMATOSOS SISTÉMICO.

CRITERIOS PARA DIAGNÓSTICO

DE LUPUS ERITEMATOSO SISTÉMICO..

INDICE DE ACTIVIDAD

DE LUPUS ERITEMATOSO SISTÉMICO - SLEDAI

\section{GASTROENTEROLOGÍA}

SANGRADO DIGESTIVO

ESCALA DE BLATCHFORD 208

ESCALA ROCKALL PARA HEMORRAGIA DIGESTIVA ALTA .211

CLASIFICACIÓN DE FORREST PARA HEMORRAGIA DIGESTIVA ALTA... .213

PUNTUACIÓN AIMS65 PARA MORTALIDAD

POR HEMORRAGIA DIGESTIVA ALTA

CIRROSIS HEPÁTICA.

ESCALA DE CHILD-PUGH.

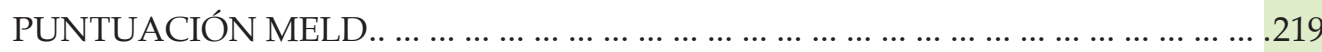

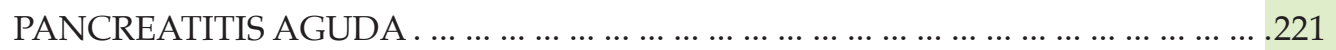

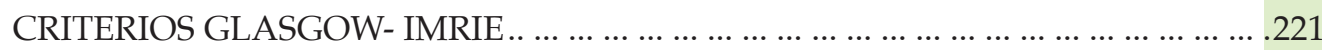

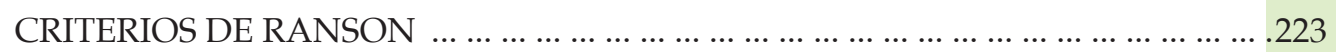

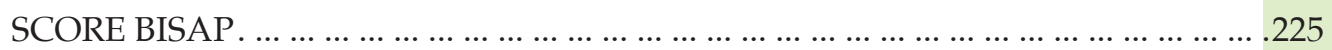


ESCALA DE MARSHALL MODIFICADA

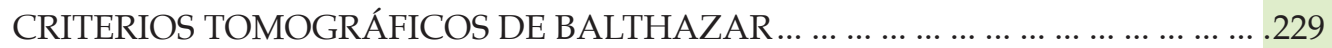

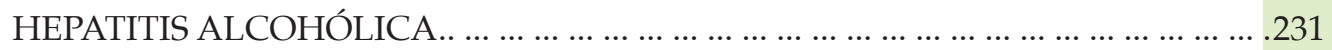

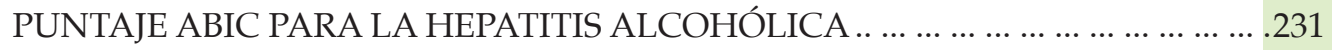

\section{INFECTOLOGÍA}

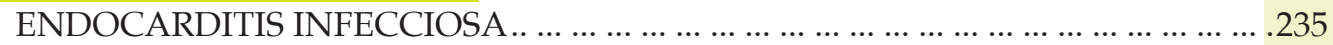

CRITERIOS DE DUKE PARA ENDOCARDITIS INFECCIOSA $\ldots \ldots \ldots \ldots \ldots \ldots \ldots . . \ldots \ldots \ldots$

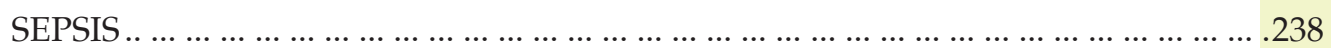

SOFA (SEQUENTIAL ORGAN FAILURE ASSESSMENT) $\ldots \ldots \ldots \ldots \ldots \ldots \ldots \ldots \ldots . . \ldots \ldots \ldots$

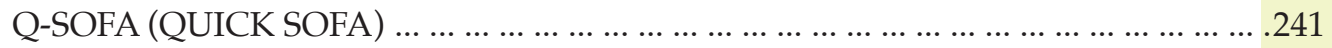

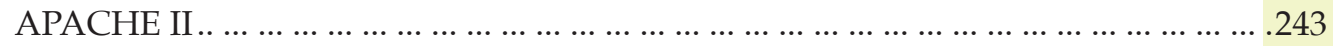

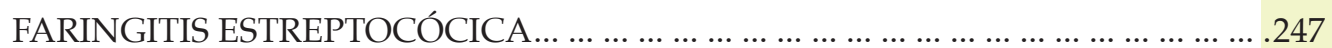

PUNTAJE DE CENTOR (MODIFICADO / MCISAAC)

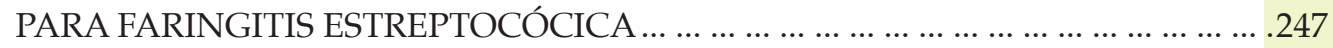

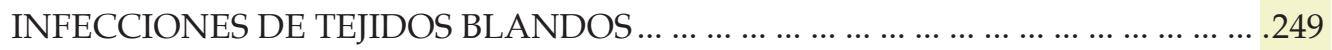

PUNTUACIÓN LRINEC PARA LA INFECCIÓN

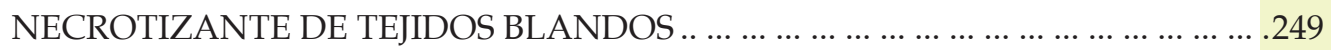

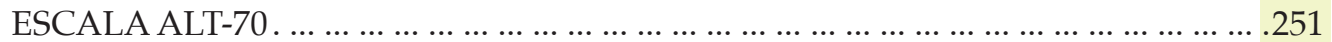

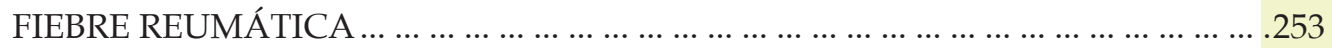

CRITERIOS DE JONES PARA DIAGNÓSTICO

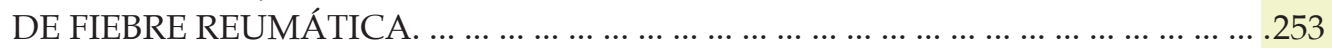

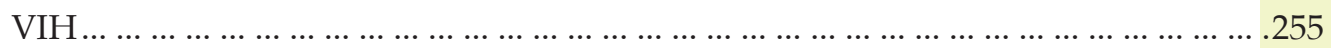

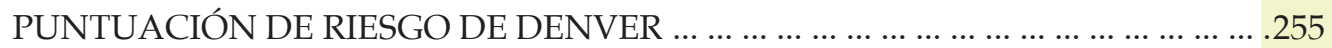

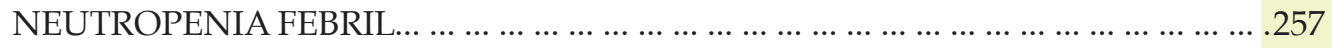

ÍNDICE CLÍNICO DE NEUTROPENIA FEBRIL ESTABLE (CISNE) … ............ . 257 


\section{HEMATOLOGÍA}

RECUENTO ABSOLUTO DE LINFOCITOS (ALC).. .266

RECUENTO ABSOLUTO DE NEUTRÓFILOS (ANC). .267

ÍNDICE ABSOLUTO DE RETICULOCITOS E

ÍNDICE DE RETICULOCITOS

ECUACIÓN DE GANZONI PARA LA ANEMIA

POR DEFICIENCIA DE HIERRO

PUNTUACIÓN DE 4TS PARA LA TROMBOCITOPENIA INDUCIDA

POR HEPARINA

OTRAS ESCALAS Y CÁLCULOS DE USO EN LA PRÁTICA CLÍNICA

ESCALA DE BRADEN PARA ÚLCERAS POR PRESIÓN.

ESCALA DE MORSE PARA RIESGO DE CAÍDA.

ESCALA DE KARNOFSKY

CÁLCULOS HEMODINÁMICOS.

\section{APÉNDICE}

I. CÁLCULOS Y ESCALAS EN LA VALORACIÓN

NUTRICIONAL DEL ADULTO Y ADULTO MAYOR .

II.CÁLCULOS Y ESCALAS EN LA UNIDAD

DE CUIDADOS INTENSIVOS.

III. ESCALA DE NEWS 2 MODIFICADA..

IV. PUNTUACIÓN DE ALERTA TEMPRANA PARA COVID-19 . . ..................... 366

V. FÓRMULAS MEDICINA BASADA EN EVIDENCIA $\ldots \ldots \ldots \ldots \ldots \ldots \ldots \ldots \ldots \ldots \ldots$ 


\section{PRÓLOGO}

Luego del lanzamiento de la obra titulada "Protocolos Terapéuticos en Medicina Interna", donde su contenido bibliográfico permitió un acercamiento a la información científica más relevante y actualizada en el campo clínico, surgió la necesidad de buscar un complemento que identifique los principales cálculos y escalas pronósticas, utilizadas frecuentemente en cada una de las subespecialidades de la medicina interna. Somos conscientes que el análisis clínico representa un proceso complejo, desde la recopilación de la información a través de la historia clínica, hasta el análisis exegético basado en problemas, con la finalidad de identificar la mejor conducta diagnóstica y terapéutica; sin embargo, en este camino es imprescindible hacer uso de escalas, fórmulas y demás cálculos validados por estudios científicos de alto impacto, para analizar probabilidades, predecir complicaciones, riesgos o simplemente evaluar la respuesta a un tratamiento determinado. Todas éstas intervenciones, nos permitirán tomar decisiones de forma oportuna, y por ende mejorar la sobrevida de nuestros pacientes. "Cálculos y escalas pronósticas en Medicina Interna", bajo ningún concepto es una herramienta aislada en la práctica clínica diaria, requiere del juicio y criterio de cada profesional o especialista en su área para la aplicación eficiente en cada paciente.

Héctor Ortega C. $M D, M P H, P h D(c)$ 


\section{AGRADECIMENTO Y DEDICATORIA}

Agradezco a mis colegas, médicos residentes y estudiantes de medicina por motivar en mí el deseo constante y permanente de alcanzar la excelencia académica, por permitirme compartir junto a ellos espacios de docencia e investigación; donde la discusión científica se vuelve el fruto de una interacción mental, organizada y sistemática. A mis pacientes, por ser la razón de ser de cada proyecto y trabajo de investigación, sin ellos, serían inertes escritos plasmados en un papel para alimentar el ego.

Dedicado a mis padres, por enseñarme el valor de la constancia y el sacrificio. A mi hijo, por permitirme robar el tiempo destinado a nuestros juegos y actividades. Y a ti, mi más ferviente admiradora y compañera de vida, por crear con tu presencia y lealtad, el prototipo de un mejor ser humano. 


\section{AUTOR-EDITOR}

\section{Héctor Fabián Ortega Castillo}

Doctorante $(\mathrm{PhD})$ en Ciencias Médicas. Universidad del Zulia/Venezuela.

Médico Especialista en Medicina Interna. Universidad Central del Ecuador.

Magíster en Salud Pública. Universidad Autónoma de los Andes.

Especialista en Gestión de Proyectos de Salud. Universidad Autónoma de los Andes.

Diploma Superior en Investigación y Proyectos. Universidad Tecnológica Equinoccial.

Diploma Superior en Docencia Universitaria en Ciencias de la Salud. Universidad

Nacional de Chimborazo(Unach). Riobamba-Ecuador.

Diploma Superior en Promoción y Prevención en Salud. Universidad Autónoma de los Andes.

Médico Tratante y Ex Jefe del Servicio de Medicina Interna del Hospital Alfredo Noboa Montenegro. Guaranda-Ecuador.

Ex Docente de la Cátedra de Farmacología y Semiología. Universidad Estatal de Bolívar. Guaranda-Ecuador.

Médico Tratante y Coordinador Pro tempore del Servicio de Clínica del Hospital General Riobamba-Instituto Ecuatoriano de Seguridad Social.

Director Técnico de Hospitalización y Ambulatorio del Hospital General RiobambaInstituto Ecuatoriano de Seguridad Social.

Director Médico Pro tempore del Hospital General Riobamba-Instituto Ecuatoriano de Seguridad Social.

Tutor de Medicina Interna del Programa de Internado Rotativo de Medicina de la Universidad Nacional de Chimborazo (Unach). Riobamba-Ecuador. 


\section{COAUTORES}

\section{Bolitigui Coulibaly}

Especialista de primer grado en Cardiología. Escuela Latinoamericana de Medicina (Habana-Cuba).

Especialista en Medicina General Integral. Escuela Latinoamericana de Medicina (Habana-Cuba).

Médico Tratante de Cardiología y ecocardiografista del Hospital Alfredo Noboa Montenegro.

\section{Francisco Villacorta}

Especialista en Medicina Crítica y Terapia Intensiva. Universidad Central del Ecuador. Magíster en Emergencias Médicas. Universidad de Guayaquil.

Médico Tratante de Terapia Intensiva del Hospital Provincial General Docente de Riobamba.

Docente de la Cátedra de Neumología y Manejo del Paciente en la Facultad de Medicina de la Universidad Nacional de Chimborazo(Unach). Riobamba-Ecuador.

\section{Johanna Elizabeth Villacrés Cervantes.}

Nutricionista-Dietista. Escuela Superior Politécnica de Chimborazo (Espoch).

Maestrante del Postgrado de Nutrición Pediátrica en la Escuela Superior Politécnica de Chimborazo (Espoch).

Nutricionista del Hospital del Día “COLPOMED”. Riobamba-Ecuador.

Jessica Marcela Monar Carrera

Médico General. Universidad Nacional de Chimborazo(Unach). Riobamba-Ecuador.

\section{María José Bahamonde Gaibor}

Médico General. Universidad Nacional de Chimborazo(Unach). Riobamba-Ecuador. Médico Residente del Hospital San Juan. Riobamba-Ecuador.

\section{Francis Valeria Pazos Raza}

Médico General. Universidad Nacional de Chimborazo(Unach). Riobamba-Ecuador. 


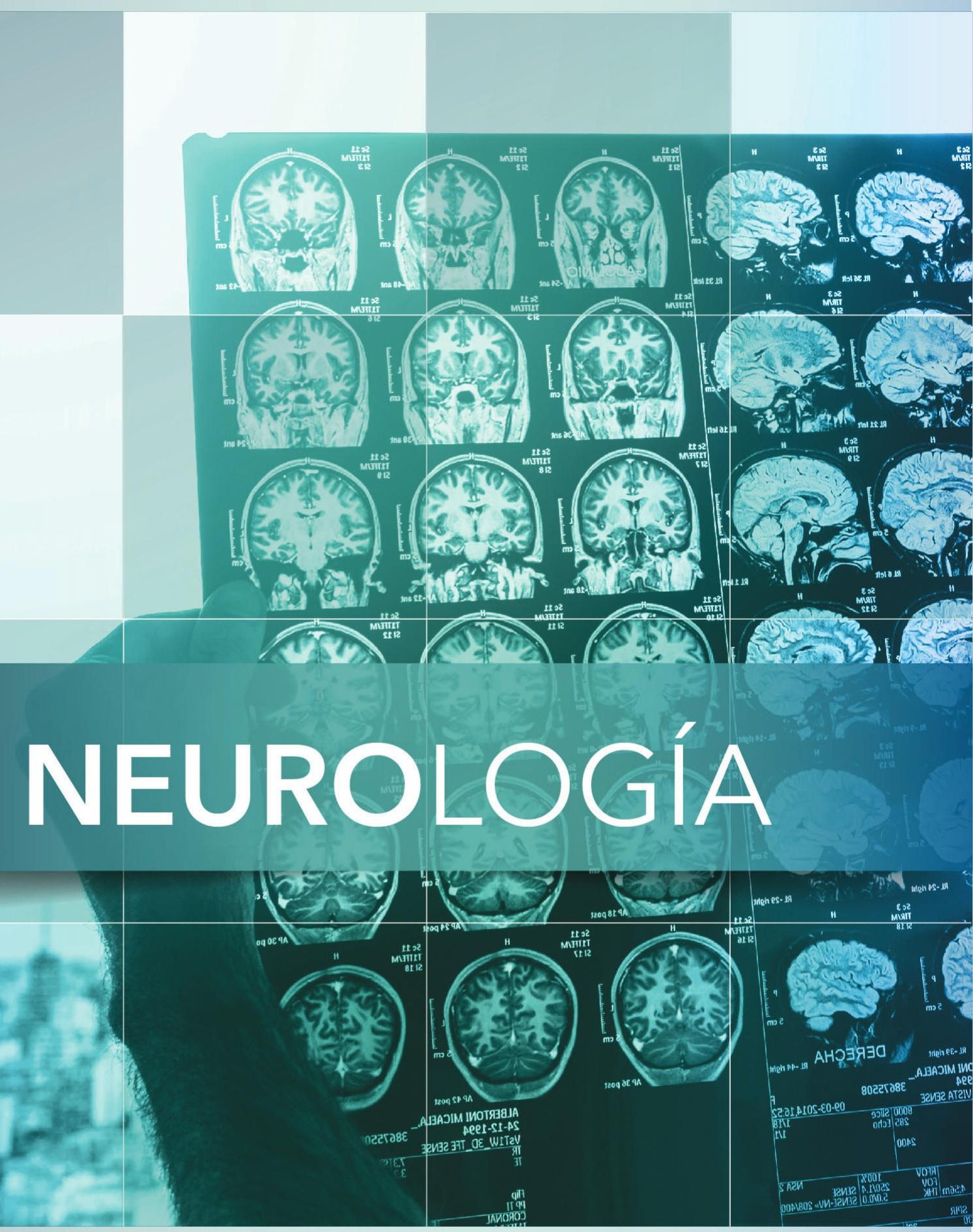


CÁculos $Y$

PROHÓSTICAS

EN MEDICINA INTERNA 


\section{ENFERMEDAD CEREBROVASCULAR HEMORRÁGICA ESCALA DE COMA DE GLASGOW}

\section{Utilidad}

La escala de coma de glasgow (GSC) es un instrumento de alta sensibilidad que permite valorar el nivel de conciencia de un paciente.

\section{Descripción}

- Se compone de 3 subescalas de observación clínica que califican de forma individual 3 aspectos de la conciencia: respuesta ocular, respuesta verbal y respuesta motora.

- La GCS se usa comúnmente en el entorno pre-hospitalario y de cuidados intensivos, así como en el curso hospitalario de un paciente para evaluar su nivel de conciencia.

- Su propósito es alertar al personal médico y de enfermería ante alguna alteración neurológica del paciente.

\section{Parámetros}

\begin{tabular}{|l|l|}
\hline RESPUESTA OCULAR & PUNTAJE \\
\hline Espontánea & 4 \\
Al estímulo verbal & 3 \\
Al dolor & 2 \\
No hay apertura ocular & 1 \\
\hline RESPUESTA VERBAL & 5 \\
\hline $\begin{array}{l}\text { Orientada } \\
\text { Confusa }\end{array}$ & 4 \\
Palabras inapropiadas & 3 \\
Sonidos incomprensibles & 2 \\
No hay respuesta verbal & 1 \\
Intubado & 1 \\
\hline
\end{tabular}




\begin{tabular}{|l|l|}
\hline RESPUESTA MOTORA & 6 \\
\hline Obedece órdenes & 5 \\
Localiza el dolor & 4 \\
Retira al dolor & 3 \\
Flexiona al dolor (decorticación) & 2 \\
Entiende al dolor (descerebración) & 1 \\
Sin respuesta & \\
\hline
\end{tabular}

\section{Interpretación}

\begin{tabular}{|l|l|}
\hline PUNTAJE & CONDICIÓN \\
\hline $\mathbf{1 3}-\mathbf{1 5}$ & daño cerebral leve \\
\hline $\mathbf{9 - 1 2}$ & daño cerebral moderado \\
\hline$\leq \mathbf{8}$ & daño cerebral severo \\
\hline
\end{tabular}

\section{Bibliografía}

\section{Original/Primaria}

1. Teasdale G, Jennett B. Valoración del coma y alteración de la conciencia. Una escala práctica. Lancet [Internet]. 1974 [citado marzo 2019]; 2 (7872): 81-4. Disponible en: https: / / www.ncbi.nlm.nih.gov/ pubmed / 4136544 .

\section{Validación}

1. Moore L, Lavoie A, Camden S, Le Sage N, Sampalis JS, Bergeron E, Abdous B. Validación estadística del Glasgow Coma Score.J Trauma [Internet]. Junio 2006 [citado marzo 2019];60(6):1238-43. Disponible en: https: / / www.ncbi.nlm.nih.gov/pubmed/16766966.

2. Teasdale G, Jennett B. Evaluación del coma y la gravedad del daño cerebral. Anestesiología [Internet]. 1978 [citado marzo de 2019]; 49:225226. Disponible en: https: / / www.ncbi.nlm.nih.gov/ pubmed/ 686455. 
3. Teasdale G, Jennett B, Murray L, Murray G. Escala de coma de Glasgow: sumar o no sumar. Lanceta [Internet]. 198317 de septiembre [citado marzo 2019]; 2 (8351): 678. Disponible en: https:/ / www.ncbi. nlm.nih.gov/pubmed/6136811.

4. Healey C, Osler TM, Rogers FB, Healey MA, Glance LG, Kilgo PD, et al. Mejora de la puntuación de la escala de coma de Glasgow: la puntuación motora sola es un mejor predictor. J Trauma [Internet]. Abril de 2003 [citado marzo 2019]; 54 (4): 671-8; discusión 678-80. Disponible en: https: / / www.ncbi.nlm.nih.gov/pubmed/12707528.

5. Green SM. Cheerio, muchacho! Despidiéndose de la Coma Scale de Glasgow. Ann Emerg Med [Internet]. Noviembre 2011[citado marzo 2019]; 58 (5): 427-30. Disponible en: https: / / www.ncbi.nlm.nih.gov / pubmed / 21803447 .

6. Middleton PM. Uso práctico de la escala de coma de Glasgow; una revisión narrativa exhaustiva de la metodología GCS. Australas Emerg Nurs J. J. Agosto [citado marzo 2019] 15 (3): 170-83. Disponible en: https: / / www.ncbi.nlm.nih.gov/ pubmed / 22947690 


\section{ESCALA ATRIA PARA RIESGO DE HEMORRAGIA}

\section{Utilidad}

Predice el riesgo de hemorragia en pacientes que toman warfarina.

\section{Descripción}

- Los riesgos y beneficios específicos de la anticoagulación, deben ser analizados cuidadosamente en todos los pacientes que son candidatos potenciales, para el tratamiento con warfarina a largo plazo.

\section{Parámetros}

\begin{tabular}{|l|l|}
\hline CRITERIO & PUNTAJE \\
\hline $\begin{array}{l}\text { Anemia } \\
\text { Hemoglobina } \\
\text { Hombre }<13 \mathrm{~g} / \mathrm{dL} \text { hombre } \\
\text { Mujer }<12 \mathrm{~g} / \mathrm{dL}\end{array}$ & 3 \\
\hline $\begin{array}{l}\text { Enfermedad renal severa } \\
\text { Velocidad de filtración glomerular } \\
<30 \text { ml / min o dependiente de diálisis }\end{array}$ & 3 \\
\hline Edad $\geq 75$ años & 2 \\
\hline Cualquier diagnóstico previo de hemorragia & 1 \\
\hline Historia de hipertensión & 1 \\
\hline
\end{tabular}

Interpretación

\begin{tabular}{|l|l|}
\hline PUNTAJE & CONDICIÓN \\
\hline$<4$ & Bajo riesgo \\
\hline 4 & Riesgo intermedio \\
\hline$>4$ & Alto riesgo \\
\hline
\end{tabular}




\section{Bibliografía}

\section{Original /Primaria}

1. Fang MC, Go AS, Chang Y, Borowsky LH, Pomernacki NK, Udaltsova $\mathrm{N}$. Un nuevo esquema de riesgo para predecir la hemorragia asociada a warfarina: el estudio ATRIA (anticoagulación y factores de riesgo en la fibrilación auricular). J Am Coll Cardiol [Internet]. 2011 [citado marzo 2019]; 58 (4): 395-401. Disponible en: http:/ / www.onlinejacc. org/content/58/4/395.

\section{Validación}

1. Roldán V, Marín F, Fernández $\mathrm{H}$, et al. El valor predictivo de las puntuaciones de hemorragia HAS-BLED y ATRIA para el riesgo de hemorragia grave en una población del "mundo real" con fibrilación auricular que recibe tratamiento anticoagulante. Chest [Internet]. 2013 [citado marzo 2019]; 143 (1): 179-184. Disponible en: https:/ / www. ncbi.nlm.nih.gov/pubmed/22722228.

2. Senoo K, Proietti M, Lane DA. Evaluación de las puntuaciones de riesgo de hemorragia HAS-BLED, ATRIA y ORBIT en pacientes con fibrilación auricular que toman warfarina. AJM Plus [Internet]. Junio 2016 [citado marzo 2019]. 129 (6): 600-607. Disponible en: https:// www.amjmed.com/ article/S0002-9343(15)00926-2 / fulltext. 


\section{ESCALA CINCINNATI PARA ACCIDENTE CEREBROVASCULAR}

\section{Utilidad}

Es una escala pre-hospitalaria que permite valorar, mediante cuatro parámetros, la posibilidad de que un paciente este sufriendo un accidente cerebrovascular (ACV).

\section{Descripción}

- La aparición repentina de alguno de estos signos es indicativo de la existencia de alguna lesión cerebral, hay que buscar asistencia médica de forma inmediata. Posibilidades de que sea una falsa alarma, pero, en todo caso, se recomienda buscar atención médica.

\section{Parámetros}

\begin{tabular}{|c|c|c|}
\hline \multirow{3}{*}{$\begin{array}{l}\text { Face } \\
\text { (Asimetría } \\
\text { facial) }\end{array}$} & \multirow{3}{*}{ 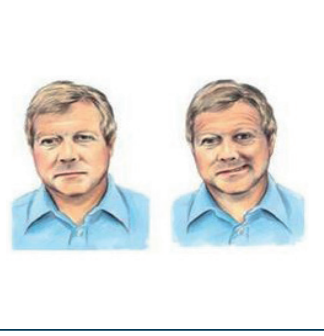 } & $\begin{array}{l}\text { Instrucción: pedir al paciente que } \\
\text { sonría. }\end{array}$ \\
\hline & & $\begin{array}{l}\text { Normal: ambos lados de la cara se } \\
\text { mueven por igual. }\end{array}$ \\
\hline & & $\begin{array}{l}\text { Anormal: un lado de la cara no se } \\
\text { mueve tan bien. }\end{array}$ \\
\hline \multirow{3}{*}{$\begin{array}{l}\text { Arms } \\
\text { (Caída del } \\
\text { brazo) }\end{array}$} & \multirow{3}{*}{ औरำ } & $\begin{array}{l}\text { Instrucción: pedir al paciente que } \\
\text { cierre los ojos y extienda ambos brazos } \\
\text { hacia afuera durante } 10 \text { segundos. }\end{array}$ \\
\hline & & $\begin{array}{l}\text { Normal: Ambos brazos se mueven } \\
\text { igual o nada en absoluto. }\end{array}$ \\
\hline & & $\begin{array}{l}\text { Anormal: Un brazo no se mueve ni se } \\
\text { desplaza hacia abajo. }\end{array}$ \\
\hline \multirow{2}{*}{$\begin{array}{l}\text { Speech } \\
\text { (Habla) }\end{array}$} & \multicolumn{2}{|c|}{$\begin{array}{l}\text { Instrucción: pedir al paciente que repita una oración } \\
\text { simple. }\end{array}$} \\
\hline & \multicolumn{2}{|c|}{$\begin{array}{l}\text { Normal: El paciente articula mal las palabras o las utiliza } \\
\text { de modo confuso o no habla. }\end{array}$} \\
\hline $\begin{array}{l}\text { Time } \\
\text { (Tiempo) }\end{array}$ & \multicolumn{2}{|c|}{$\begin{array}{l}\text { Anormal: Si estos signos aparecen repentinamente o están } \\
\text { acompañados por la pérdida de la visión, la pérdida de } \\
\text { equilibrio con mareos o un intenso dolor de cabeza, se } \\
\text { necesita asistencia médica de forma inmediata. }\end{array}$} \\
\hline
\end{tabular}




\section{Interpretación}

\section{RESULTADO PROBABILIDAD DE ACV}

\begin{tabular}{|l|l|}
\hline 1 signo positivo & $72 \%$ \\
\hline 3 signos positivos & $>85 \%$ \\
\hline
\end{tabular}

\section{Bibliografía}

\section{Original/Primaria}

1. Kothari RU, Pancioli A, Liu T, Brott T, Broderick J. Cincinnati Prehospital Stroke Scale: reproducibilidad y validez. Ann Emerg Med [Internet]. Abril de 1999 [citado marzo 2019]; 33 (4): 373-8. Disponible en: https: / / www.ncbi.nlm.nih.gov/ pubmed / 10092713.

2. Kummer BR, Gialdini G, Sevush JL, Kamel H, Patsalides A. Validación externa de la escala de severidad de ictus prehospitalaria de Cincinnati. J Stroke Cerebrovasc Dis [Internet]. Mayo 2016 [citado marzo 2019]; 25 (5): 1270-1274. Disponible en: https://www.ncbi.nlm.nih.gov/ pubmed/26971037.

3. Katz BS, McMullan JT, Sucharew H, Adeoye O, Broderick JP. Diseño y validación de una escala prehospitalaria para predecir la gravedad del accidente cerebrovascular: Escala de severidad de ictus prehospitalaria de Cincinnati. Stroke [Internet]. Junio 2015 [citado marzo 2019] ;46(6):1508-12. Disponible en: https://www.ncbi.nlm. nih.gov/pubmed/25899242. 


\section{ESCALA ICH (INTRACEREBRAL HEMORRHAGE)}

\section{Utilidad}

El score para hemorragia intracerebral (ICH), es una escala de clasificación clínica que permite la estratificación del riesgo de mortalidad, en 30 días, en pacientes que presentan $\mathrm{ICH}$; está basado en la edad y hallazgos de la tomografía.

\section{Descripción}

- La escala ICH permite una calificación clínica estandarizada y consistente para ICH, mejorando así la comunicación entre profesionales de la medicina.

- Esta escala califica la gravedad de la ICH y la mortalidad subsiguiente en 30 días, lo que ayuda a guiar las conversaciones sobre los objetivos de atención con los familiares de los pacientes.

- Este puntaje se usa a menudo junto con el puntaje FUNC, que indica la probabilidad de independencia funcional de un paciente 90 días después de la ICH.

\section{Parámetros}

\begin{tabular}{|l|l|}
\hline PUNTAJE GSC & PUNTAJE \\
\hline $3-4$ & 2 \\
$5-12$ & 1 \\
$13-15$ & 0 \\
\hline EDAD & \\
\hline Mayor o igual a 80 años & 1 \\
Menor a 80 años & 0 \\
\hline UBICACIÓN & \\
\hline Infratentorial & 1 \\
Supratentorial & 0 \\
\hline
\end{tabular}




\begin{tabular}{|l|l|}
\hline VOLUMEN & \\
\hline Mayor o igual a $30 \mathrm{~cm}^{3}$ & 1 \\
Menor a $30 \mathrm{~mm}^{3}$ & 0 \\
\hline HEMORRAGIA INTRAVENTRICULAR & \\
\hline Presente & 1 \\
Ausente & 0 \\
\hline
\end{tabular}

ICH: hemorragia intracerebral; GSC: escala de coma de glasgow.

\section{Interpretación}

\begin{tabular}{|c|c|}
\hline PUNTUACIÓN ICH & TASA DE MORTALIDAD \\
\hline $\mathbf{0}$ & $0 \%$ \\
\hline $\mathbf{1}$ & $13 \%$ \\
\hline $\mathbf{2}$ & $26 \%$ \\
\hline $\mathbf{3}$ & $72 \%$ \\
\hline $\mathbf{4}$ & $94 \%$ \\
\hline $\mathbf{5}$ & $100 \%$ \\
\hline $\mathbf{6}$ & $100 \%$ \\
\hline
\end{tabular}

\section{Bibliografía}

\section{Original/Primaria}

1. Hemphill JC 3rd, Bonovich DC, Besmertis L, Manley GT, Johnston SC. La puntuación ICH: una escala de calificación simple y confiable para la hemorragia intracerebral. Stroke [Internet]. Abril 2001[citado marzo 2019]; 32 (4): 891-7. Disponible en: https://www.ncbi.nlm.nih.gov/ pubmed/11283388.

\section{Validación}

1. Clarke JL, Johnston SC, Farrant M, Bernstein R, Tong D, Hemphill JC 3rd. Validación externa de la puntuación ICH. Cuidado del neurocrito [Internet]. 2004 [citado marzo 2019];1(1):53-60. Disponible en: https: / / www.ncbi.nlm.nih.gov/pubmed/16174898.

2. Baharoglu MI, Cordonnier C, Al-shahi salman R, et al. Transfusión de plaquetas versus atención estándar después de un accidente cerebrovascular agudo debido a hemorragia cerebral espontánea 
asociada con la terapia antiplaquetaria (PATCH): un ensayo aleatorizado, abierto, de fase 3. Lancet [Internet]. 2016 [citado marzo 2019];387(10038):2605-13. Disponible en: https://www.thelancet. com/journals / lancet/ article/ PIIS0140-6736(16)30392-0 / fulltext. 


\section{Utilidad}

La escala FUNC permite identificar a los pacientes con hemorragia intracerebral ( $\mathrm{ICH})$ que alcanzarán la independencia funcional a los 90 días de una ICH, además ayuda en la toma de decisiones clínicas.

\section{Descripción}

- Compuesta por 5 parámetros, se usa cuando se realiza el diagnóstico de ICH, más no como un marcador del estado neurológico.

- Se recomienda usar solo en pacientes con hemorragia intracerebral primaria. No en pacientes con ICH por traumatismo.

\section{Parámetros}

\begin{tabular}{|l|l|l|}
\hline CRITERIO & CARACTERÍSTICAS & PUNTAJE \\
\hline \multirow{2}{*}{ Volumen ICH $\left(\mathrm{cm}^{3}\right)$} & $<30$ & 4 \\
& $30-60$ & 2 \\
\hline \multirow{2}{*}{ Edad } & $>60$ & 0 \\
\hline \multirow{3}{*}{ Ubicación ICH } & $<70$ & 2 \\
& $70-79$ & 1 \\
\hline Puntaje GCS & $\geq 80$ & 0 \\
\hline \multirow{2}{*}{ Deterioro congnitivo pre-ICH } & Lobar & 2 \\
& Profundo & 1 \\
& Si & 0 \\
\hline ICH: Hratentorial & 0 \\
\hline
\end{tabular}

ICH: Hemorragia Intracerebral; GSC: Escala de Coma de Glasgow. 


\section{Interpretación}

\begin{tabular}{|c|c|}
\hline PUNTUACIÓN & INDEPENDENCIA FUNCIONAL A 90 DÍAS \\
\hline $\mathbf{0 - 4}$ & $0 \%$ \\
\hline $\mathbf{5 - 7}$ & $1-20 \%$ \\
\hline $\mathbf{8}$ & $21-60 \%$ \\
\hline $\mathbf{9 - 1 0}$ & $61-80 \%$ \\
\hline $\mathbf{1 1}$ & $81-100 \%$ \\
\hline
\end{tabular}

\section{Bibliografía}

\section{Original/Primaria}

1. Rost NS, Smith EE, Snider RW et. al. Predicción del resultado funcional en pacientes con hemorragia intracerebral primaria: The FUNC Score. Stroke [Internet]. 2008 [citado marzo 2019]; 39:2304-2309. Disponible en: https://www.ahajournals.org/doi/full/10.1161/ strokeaha.107.512202.

\section{Validación}

1. Garrett JS, Zarghouni M, Layton KF, Graybeal D, Daoud YA. Validation of clinical prediction scores in patients with primary intracerebral hemorrhage. Neurocrit Care [Internet]. Diciembre 2013 [citado marzo 2019];19(3):329-35. Disponible en : https: / / www.ncbi.nlm.nih.gov/ pubmed / 24132566. 


\section{Utilidad}

Es una escala que clasifica la severidad de la hemorragia subaracnoidea por aneurisma para predecir la mortalidad.

\section{Descripción}

- Se recomienda usar esta escala al ingreso del paciente, debido a que es netamente clínica, y puede cambiar durante el curso hospitalario.

- La escala no se aplica a la hemorragia subaracnoidea por traumatismo, malformaciones arteriovenosas, angiomas cavernosos, fístulas arteriovenosas durales, trombosis venosa cortical o sinusal, aneurismas micóticos o émbolos sépticos con transformación hemorrágica.

- Los grados más altos, que reflejan una gravedad de la hemorragia y una disfunción neurológica progresivamente mayor, se asocian con una mayor mortalidad general.

\section{Parámetros}

\section{SIGNOS/SÍNTOMAS}

PUNTAJE

Paciente asintomático, dolor de cabeza leve, ligera rigidez de nuca.

Dolor de cabeza moderado a fuerte, rigidez de nuca sin déficit neurológico fuera de la parálisis del nervio craneal.

Somnolencia, confusión, obnubilación, déficit neurológico focal leve.

Estupor hemiparesia de moderada a severa, posible rigidez de descerebración o trastornos vegetativos.

Coma profundo, signos de descerebración.

1

2

3

4 5 


\section{Interpretación}

\begin{tabular}{|c|c|c|}
\hline PUNTAJE & GRADO & MORTALIDAD \\
\hline $\mathbf{1}$ & I & $30 \%$ \\
\hline $\mathbf{2}$ & II & $40 \%$ \\
\hline $\mathbf{3}$ & III & $50 \%$ \\
\hline $\mathbf{4}$ & IV & $80 \%$ \\
\hline $\mathbf{5}$ & V & $90 \%$ \\
\hline
\end{tabular}

\section{Bibliografía}

\section{Original/Primaria}

1. Hunt WE, Hess RM. Riesgo quirúrgico relacionado con el tiempo de intervención en la reparación de aneurismas intracraneales. J Neurosurg [Internet] 1968 Enero [citado marzo 2019]; 28 (1): 14-20. Disponible en: https: / www.ncbi.nlm.nih.gov/pubmed/5635959.

\section{Validación}

1. Oshiro EM, Walter KA, Piantadosi S, Witham TF, Tamargo RJ. Un nuevo sistema de clasificación de hemorragia subaracnoidea basado en la escala de coma de Glasgow: una comparación con las escalas de Hunt y Hess y la Federación Mundial de Cirujanos Neurológicos en una serie clínica. Neurosurgery [Internet]. 1997 [citado marzo 2019]; 4: 140-148. Disponible en: https://www.ncbi.nlm.nih.gov/ pubmed/9218306.

2. Aulmann C, Steudl WI, Feldmann U. Validación de la precisión pronóstica de las escalas de ingreso neuroquirúrgico tras la rotura de aneurismas cerebrales. Zentralbl Neurochir [Internet]. 1998 [citado marzo 2018]; 59 (3): 171-80. Disponible en: https:/ /www.ncbi.nlm. nih.gov/pubmed/9816668.

3. Degen LA, Dorhout SM, Alegra A, Rinkel GJ. Variabilidad interobservador de las escalas de calificación para la hemorragia subaracnoidea aneurismática. Stroke [Internet]. 2011 [citado marzo 2019]; 42: 1546-1549. Disponible en: https:/ / www.ahajournals.org/ doi/full/10.1161/strokeaha.110.601211. 


\section{Utilidad}

LaEscala de Rankin modificada(ERmo mRS) mideel gradodeincapacidad o dependencia en las actividades diarias de las personas que han sufrido un accidente cerebrovascular $\mathrm{u}$ otras causas de discapacidad neurológica.

\section{Descripción}

- La ERm se ha convertido en la herramienta más usada para medir los resultados de las pruebas clínicas realizadas en pacientes con accidentes cerebrovasculares.

\section{Parámetros}

\begin{tabular}{|l|c|}
\hline DESCRIPCIÓN & PUNTUACIÓN \\
\hline Sin síntomas en absoluto. & $\mathbf{1}$ \\
\hline $\begin{array}{l}\text { Sin discapacidad significativa a pesar de los síntomas; } \\
\text { capaz de realizar todos los deberes y actividades } \\
\text { habituales. }\end{array}$ & $\mathbf{2}$ \\
\hline $\begin{array}{l}\text { Discapacidad leve; incapaz de llevar a cabo todas } \\
\text { las actividades anteriores, pero capaz de cuidar sus } \\
\text { propios asuntos sin ayuda. }\end{array}$ & $\mathbf{3}$ \\
\hline $\begin{array}{l}\text { Discapacidad moderada; requiere ayuda, pero puede } \\
\text { caminar sin ayuda. }\end{array}$ & $\mathbf{4}$ \\
\hline $\begin{array}{l}\text { Discapacidad moderadamente grave; incapaz de } \\
\text { caminar y atender las necesidades corporales sin } \\
\text { ayuda. }\end{array}$ & $\mathbf{5}$ \\
\hline $\begin{array}{l}\text { Discapacidad severa; estar en cama, incontinente } \\
\text { y que requiere cuidados y atención de enfermería } \\
\text { constantes. }\end{array}$ & $\mathbf{6}$ \\
\hline Muerto. & \\
\hline
\end{tabular}




\section{Interpretación}

\begin{tabular}{|c|c|}
\hline PUNTUACIÓN & CONDICIÓN \\
\hline 0 & Asintomático \\
\hline 1 & Muy leve \\
\hline 2 & Leve \\
\hline 3 & Moderada \\
\hline 4 & Moderadamente grave \\
\hline 5 & Grave \\
\hline 6 & Muerte \\
\hline
\end{tabular}

\section{Bibliografía}

\section{Original/Primaria}

van Swieten JC, Koudstaal PJ, Visser MC, Schouten HJ, van Gijn J. Acuerdo interobservador para la evaluación de la discapacidad en pacientes con accidente cerebrovascular. Stroke [Internet]. 1988 mayo [citado marzo 2019]; 19(5):604-7. Disponible en: https://www.ncbi.nlm.nih.gov/ pubmed/3363593.

\section{Validación}

Bancos JL, Marotta CA. La validez de los resultados y la fiabilidad de la escala modificada de Rankin: implicaciones para los ensayos clínicos de accidente cerebrovascular: una revisión de la literatura y síntesis. Stroke [Internet]. 2007 marzo [citado marzo 2019];38(3):1091-6. Disponible en: https: / / www.ncbi.nlm.nih.gov/pubmed/17272767.

Wilson JT, Hareendran A, Grant M, Baird T, Schulz UG, Muir KW, Bone I. Mejora de la evaluación de los resultados en el accidente cerebrovascular: uso de una entrevista estructurada para asignar calificaciones en la escala de Rankin modificada. Stroke [Internet]. Septiembre de 2002 [citado marzo 2019]; 33 (9): 2243-6. Disponible en: https:// www.ncbi.nlm.nih. gov/pubmed/12215594.

Bruno A, Shah N, Lin C, Close B, Hess DC, Davis K. Mejora de la evaluación de la escala de Rankin modificada con un cuestionario simplificado. Stroke. 2010 mayo [citado marzo 2019];41(5):1048-50. Disponible en: https: / / www.ncbi.nlm.nih.gov/pubmed/20224060. 
Wilson JT, Hareendran A, Hendry A, Potter J, Bone I, Muir KW. Fiabilidad de la escala de Rankin modificada en múltiples evaluadores: beneficios de una entrevista estructurada. Stroke [Internet]. Abril de 2005 [citado marzo 2019]; 36 (4): 777-81. Disponible en: https:/ / www.ncbi.nlm.nih. gov/pubmed/15718510.

Quinn TJ, Dawson J, Walters MR, Lees KR. Fiabilidad de la escala de Rankin modificada: una revisión sistemática. Stroke [Internet]. Octubre 2009 [citado marzo 2019];40(10):3393-5. Disponible en: https://www. ncbi.nlm.nih.gov/pubmed/19679846. 


\section{CRISIS CONVULSIVAS}

ESCALA PARA ESTIMAR LA SEVERIDAD DEL ESTATUS EPILÉPTICO

\section{Utilidad}

La escala STESS (Status Epilepticus Severity Score) proporciona una estimación de la mortalidad hospitalaria de cada paciente, basándose en cuatro predictores de resultados (edad, historial de convulsiones, tipo de crisis, grado de deterioro de la conciencia).

Parámetros

\begin{tabular}{|l|l|c|}
\hline CRITERIO & CARACTERÍSTICAS & PUNTAJE \\
\hline $\begin{array}{l}\text { Estado de } \\
\text { conciencia }\end{array}$ & Alerta o somnoliento/confuso. & 0 \\
& Estupor o coma. & 1 \\
\hline $\begin{array}{l}\text { Peor tipo de } \\
\text { convulsiones } \\
\text { ausencia, mioclonía. }\end{array}$ & $\begin{array}{l}\text { Convulsión generalizada. } \\
\text { Estado epiléptico no convulsivo en } \\
\text { coma. }\end{array}$ & 2 \\
\hline $\begin{array}{l}\text { Edad } \\
\text { Historia previa } \\
\text { de epilepsia }\end{array}$ & $\begin{array}{l}\text { SÍ } \\
\text { No o no conoride 65 años. }\end{array}$ & 0 \\
\hline
\end{tabular}

\section{Interpretación}

\begin{tabular}{|l|l|}
\hline PUNTUACIÓN & CONDICIÓN \\
\hline $0-2$ & Favorable \\
\hline $3-6$ & Desfavorable \\
\hline
\end{tabular}




\section{Bibliografía}

\section{Original/Primaria}

1. Rossetti AO, Logroscino G, Milligan TA, Michaelides C, Ruffieux C, Bromfield EB. Status Epilepticus Severity Score (STESS): una herramienta para orientar la estrategia de tratamiento temprano. J Neurol [Internet]. Octubre 2008 [citado marzo 2019]; 255(10):1561-6. Disponible en: https:/ / www.ncbi.nlm.nih.gov/pubmed/18769858. 


\section{ENFERMEDAD CEREBROVASCULAR ISQUÉMICA}

\section{ESCALA NIHSS PARA ACV AGUDO}

\section{Utilidad}

La escala NIHSS (National Institute of Health Stroke Scale) ayuda a estimar la gravedad de un accidente cerebrovascular en el contexto agudo.

\section{Descripción}

- Las puntuaciones en aumento, indican un accidente cerebrovascular más grave y se ha demostrado que, se correlaciona con el tamaño del infarto en la evaluación de TAC y RMN.

- El puntaje de esta escala, cuando se evalúa dentro de las primeras 48 horas posteriores a un accidente cerebrovascular, se correlacionan con los resultados clínicos a los 3 meses y al año.

- Los pacientes con una puntuación total de 4 o menos, generalmente tienen resultados clínicos favorables y tienen una alta probabilidad de independencia funcional independientemente del tratamiento.

\section{Parámetros}

\begin{tabular}{|c|c|c|}
\hline ASPECTOS & OPCIONES DE RESPUESTA & PTS. \\
\hline $\begin{array}{l}\text { Nivel de conciencia } \\
\text { (NC) }\end{array}$ & $\begin{array}{l}\text { Alerta } \\
\text { No alerta (mínimos estímulos } \\
\text { verbales) } \\
\text { No alerta (estímulos repetidos } \\
\text { o dolorosos) } \\
\text { Respuesta reflejas }\end{array}$ & $\begin{array}{l}0 \\
1 \\
2 \\
3\end{array}$ \\
\hline $\begin{array}{l}\text { NC-preguntas } \\
\text { 1. ¿En qué mes } \\
\text { estamos? } \\
\text { 2. ¿Qué edad tiene? }\end{array}$ & $\begin{array}{l}\text { Ambas respuestas correctas } \\
\text { Una respuesta correcta (o } \\
\text { disartria) } \\
\begin{array}{l}\text { Ninguna respuesta correcta } \\
\text { (cafasia) }\end{array}\end{array}$ & $\begin{array}{l}0 \\
1 \\
2\end{array}$ \\
\hline
\end{tabular}




\begin{tabular}{|c|c|c|}
\hline $\begin{array}{l}\text { NC-órdenes } \\
\text { 1. Cierre los ojos } \\
\text { 2. Abra y cierre la } \\
\text { mano } \\
\text { 3. Mirada }\end{array}$ & $\begin{array}{l}\text { Ambas órdenes correctas } \\
\text { Una orden correcta } \\
\text { Ninguna orden correcta } \\
\text { Normal } \\
\text { Parálisis parcial de la mirada } \\
\begin{array}{l}\text { Desviación forzado de la } \\
\text { mirada }\end{array}\end{array}$ & $\begin{array}{l}0 \\
1 \\
2 \\
0 \\
1 \\
2\end{array}$ \\
\hline Campo visual & $\begin{array}{l}\text { Normal } \\
\text { Hemianopsia parcial } \\
\text { Hemianopsia completa } \\
\text { Ceguera }\end{array}$ & $\begin{array}{l}0 \\
1 \\
2 \\
3\end{array}$ \\
\hline Paresia facial & $\begin{array}{l}\text { Movilidad normal } \\
\text { Paresia menor } \\
\text { Paresia parcial } \\
\begin{array}{l}\text { Parálisis completa de la } \\
\text { hemicara }\end{array}\end{array}$ & $\begin{array}{l}0 \\
1 \\
2 \\
3\end{array}$ \\
\hline $\begin{array}{l}\text { Miembro superior } \\
\text { Derecho / Miembro } \\
\text { Superior izquierdo }\end{array}$ & $\begin{array}{l}\text { No caída del miembro } \\
\text { Caída en menos de } 10 \text { segundos } \\
\text { Esfuerzo contra gravedad } \\
\text { Movimiento en plano } \\
\text { horizontal } \\
\text { No movimiento }\end{array}$ & $\begin{array}{l}0 / 0 \\
1 / 1 \\
2 / 2 \\
3 / 3 \\
4 / 4\end{array}$ \\
\hline $\begin{array}{l}\text { Miembro inferior } \\
\text { Derecho/ Miembro } \\
\text { Inferior izquierda }\end{array}$ & $\begin{array}{l}\text { No caída del miembro } \\
\text { Caída en menos de } 5 \text { segundos } \\
\text { Esfuerzo contra la gravedad } \\
\text { Movimiento en pleno } \\
\text { horizontal } \\
\text { No movimiento }\end{array}$ & $\begin{array}{l}0 / 0 \\
1 / 1 \\
2 / 2 \\
3 / 3 \\
4 / 4\end{array}$ \\
\hline
\end{tabular}




\begin{tabular}{|c|c|c|}
\hline Ataxia en miembro & $\begin{array}{l}\text { Ausente } \\
\text { Presente en } 1 \text { extremidad } \\
\text { En } 2 \text { o más extremidades }\end{array}$ & $\begin{array}{l}0 \\
1 \\
2\end{array}$ \\
\hline Exploración sensitiva & $\begin{array}{l}\text { Normal } \\
\text { Pérdida entre ligera a moderada } \\
\text { Pérdida entre grave y total }\end{array}$ & $\begin{array}{l}0 \\
1 \\
2\end{array}$ \\
\hline Lenguaje & $\begin{array}{l}\text { Normal } \\
\text { Afasia ligera a moderada } \\
\text { Afasia grave } \\
\text { Afasia global }\end{array}$ & $\begin{array}{l}0 \\
1 \\
2 \\
3\end{array}$ \\
\hline Disartria & $\begin{array}{l}\text { Normal } \\
\text { Ligera a moderada } \\
\text { Grave a anartria }\end{array}$ & $\begin{array}{l}0 \\
1 \\
2\end{array}$ \\
\hline Extinción e inatención & $\begin{array}{l}\text { Normal } \\
\text { Extinción parcial } \\
\text { Extinción completa }\end{array}$ & $\begin{array}{l}0 \\
1 \\
2\end{array}$ \\
\hline \multicolumn{2}{|l|}{ PUNTUACIÓN TOTAL } & $\begin{array}{c}0-42 \\
\text { PUNTOS }\end{array}$ \\
\hline
\end{tabular}

Interpretación

\begin{tabular}{|l|l|}
\hline PUNTUACIÓN & CONDICIÓN \\
\hline $\mathbf{0}$ & Sin accidente cerebrovascular \\
\hline $\mathbf{1 - 4}$ & Accidente cerebrovascular menor \\
\hline $\mathbf{5 - 1 5}$ & Accidente cerebrovascular moderado \\
\hline $\mathbf{1 6 - 2 0}$ & Accidente cerebrovascular grave \\
\hline $\mathbf{2} 21$ & Accidente cerebrovascular muy grave \\
\hline
\end{tabular}




\section{Bibliografía}

\section{Original/Primaria}

1. Lyden P, Brott T, Tilley B, et al. Improved reliability of the NIH Stroke Scale using video training. NINDS TPA Stroke Study Group. Stroke [Internet] 1994 [citado marzo 2019]; 25: 2220-2226. Disponible en: https: / / www.ncbi.nlm.nih.gov/pubmed/7974549.

\section{Validación}

1. Johnston KC, Connors AF Jr, Wagner DP, Haley EC Jr. Predicción del resultado en el accidente cerebrovascular isquémico: validación externa de los modelos de riesgo predictivo. Stroke [Internet]. 2003 enero [citado marzo 3019]; 34 (1): 200-2. Disponible en: https: / / www. ncbi.nlm.nih.gov/pubmed/12511774.

2. Schlegel D, Kolb SJ, Luciano JM, Tovar JM, Cucchiara BL, Liebeskind DS, et al. Utilidad de la NIH Stroke Scale como predictor de la disposición hospitalaria. Stroke [Internet]. 2003 enero [citado marzo 2019]; 34(1):134-7. Disponible en: https://www.ncbi.nlm.nih.gov/ pubmed/12511764.

3. Rundek T, Mast H, Hartmann A, Boden-Albala B, Lennihan L, Lin IF, et al. Predictores del uso de recursos después de una hospitalización aguda: el Estudio de Accidentes Cerebrovasculares del Norte de Manhattan. Neurology [Internet]. 200024 de octubre [citado marzo 2019]; 55(8):1180-7. Disponible en: https://www.ncbi.nlm.nih.gov/ pubmed / 11071497 .

4. Appelros P, Terént A. Características de la Escala de accidentes cerebrovasculares del Instituto Nacional de Salud: resultados de una cohorte poblacional de accidentes cerebrovasculares al inicio del estudio y después de un año. Cerebrovasc Dis [Internet]. 2004 [citado marzo 2019]; 17(1):21-7. Disponible en: https://www.ncbi.nlm.nih. gov/pubmed / 14530634 . 


\section{ESCALA ABCD ${ }^{2}$ PARA RIESGO DE ACCIDENTE CEREBROVASCULAR ISQUÉMICO POSTERIOR A UN ACCIDENTE ISQUÉMICO TRANSITORIO}

\section{Utilidad}

Calcula el riesgo de accidente cerebrovascular dentro de los primeros días, después de un accidente isquémico transitorio (AIT).

\section{Descripción}

- La puntuación $\mathrm{ABCD}^{2}$ no debe utilizarse como sustituto del juicio clínico o la toma de decisiones.

- Se ha demostrado que tiene una menor precisión cuando es utilizado por médicos no especialistas.

\section{Parámetros}

\begin{tabular}{|c|c|c|c|}
\hline \multicolumn{2}{|c|}{ CRITERIO } & CARACTERÍSTICAS & PUNTAJE \\
\hline A & Edad & Mayor o igual a 60 años & 1 \\
\hline B & $\begin{array}{l}\text { Presión } \\
\text { sanguínea }\end{array}$ & $\geq 140 / 90 \mathrm{mmHg}$ & 1 \\
\hline C & $\begin{array}{l}\text { Características } \\
\text { clínicas del AIT }\end{array}$ & $\begin{array}{l}\text { Debilidad unilateral } \\
\text { Alteración del lenguaje sin } \\
\text { debilidad } \\
\text { Otros síntomas }\end{array}$ & $\begin{array}{l}2 \\
1 \\
0\end{array}$ \\
\hline \multirow[t]{2}{*}{ D } & $\begin{array}{l}\text { Duración de } \\
\text { síntomas }\end{array}$ & $\begin{array}{l}\geq 60 \text { minutos } \\
10-59 \text { minutos } \\
<10 \text { minutos }\end{array}$ & $\begin{array}{l}2 \\
1 \\
0\end{array}$ \\
\hline & $\begin{array}{l}\text { Diabetes } \\
\text { mellitus } 2\end{array}$ & & 1 \\
\hline
\end{tabular}

AIT: accidente isquémico transitorio. 


\begin{tabular}{|l|l|l|l|l|}
\hline \multirow{2}{*}{ Puntuación } & \multirow{2}{*}{ Condición } & \multicolumn{3}{|l|}{ Riesgo de Evento isquémico } \\
\cline { 3 - 5 } & & $\mathbf{2}$ días & $\mathbf{7}$ días & $\mathbf{9 0}$ días \\
\hline $\mathbf{0 - 3}$ & Bajo riesgo & $1.0 \%$ & $1.2 \%$ & $3.1 \%$ \\
\hline $\mathbf{4 - 5}$ & Moderado riesgo & $4.1 \%$ & $5.9 \%$ & $9.8 \%$ \\
\hline $\mathbf{6 - 7}$ & Alto riesgo & 8.1 & $11.7 \%$ & $17.8 \%$ \\
\hline
\end{tabular}

\section{Bibliografía}

\section{Original/Primaria}

1. Johnston SC, Rothwell PM, Nguyen-Huynh MN, Giles MF, Elkins JS, Bernstein $\mathrm{AL}$, et al. Validación y refinamiento de las puntuaciones para predecir el riesgo de accidente cerebrovascular muy temprano después de un ataque isquémico transitorio. Lancet [Internet]. 27 de enero de 2007 [citado marzo 2019]; 369 (9558): 283-92. Disponible en: https: / / www.ncbi.nlm.nih.gov/ pubmed/17258668.

\section{Validación}

1. Josephson SA, Sidney S, Pham TN, Bernstein AL, Johnston SC. Una puntuación ABCD2 más alta predice que los pacientes tienen más probabilidades de tener un ataque isquémico transitorio real. Stroke [Internet]. 2008 noviembre [citado marzo 2019];39(11):3096-8. Disponible en: https: / / www.ncbi.nlm.nih.gov/ pubmed/18688003.

2. Rothwell PM, Giles MF, Flossmann E, Lovelock CE, Redgrave JN, Warlow $\mathrm{CP}$, et al. Una puntuación simple (ABCD) para identificar individuos con alto riesgo temprano de accidente cerebrovascular después de un ataque isquémico transitorio. Lancet [Internet]. Julio 2005 [citado marzo 2019];366(9479): 29-36. Disponible en: https:// www.ncbi.nlm.nih.gov/pubmed/15993230.

3. Sanders LM, Srikanth VK, Blacker DJ, Jolley DJ, Cooper KA, Phan TG. Rendimiento de la puntuación ABCD2 para el riesgo de accidente cerebrovascular después del AIT: metanálisis y modelos de probabilidad. Neurology [Internet]. Septiembre 2012 [citado marzo 
2019].;79(10):971-80. Disponible en: https: / / www.ncbi.nlm.nih.gov/ pubmed / 22700810.

4. Stead LG, Suravaram S, Bellolio MF, Enduri S, Rabinstein A, Gilmore $\mathrm{RM}$ et al. Una evaluación del valor incremental de la puntuación ABCD2 en la evaluación del departamento de emergencia del ataque isquémico transitorio. Ann Emerg Med [Internet]. Enero 2011 [citado marzo 2019]; 57 (1): 46-51. Disponible en: https: / / www.ncbi.nlm.nih. gov / pubmed / 20855130

5. Perry JJ, Sharma M, Sivilotti ML, Sutherland J, Symington C, Worster A, Émond M, et al. Validación prospectiva de la puntuación ABCD2 para pacientes en el servicio de urgencias con ataque isquémico transitorio. CMAJ [Internet]. Julio 2011 [citado marzo 2019];183(10):1137-45. Disponible en: https: / / www.ncbi.nlm.nih.gov/pubmed/21646462. 


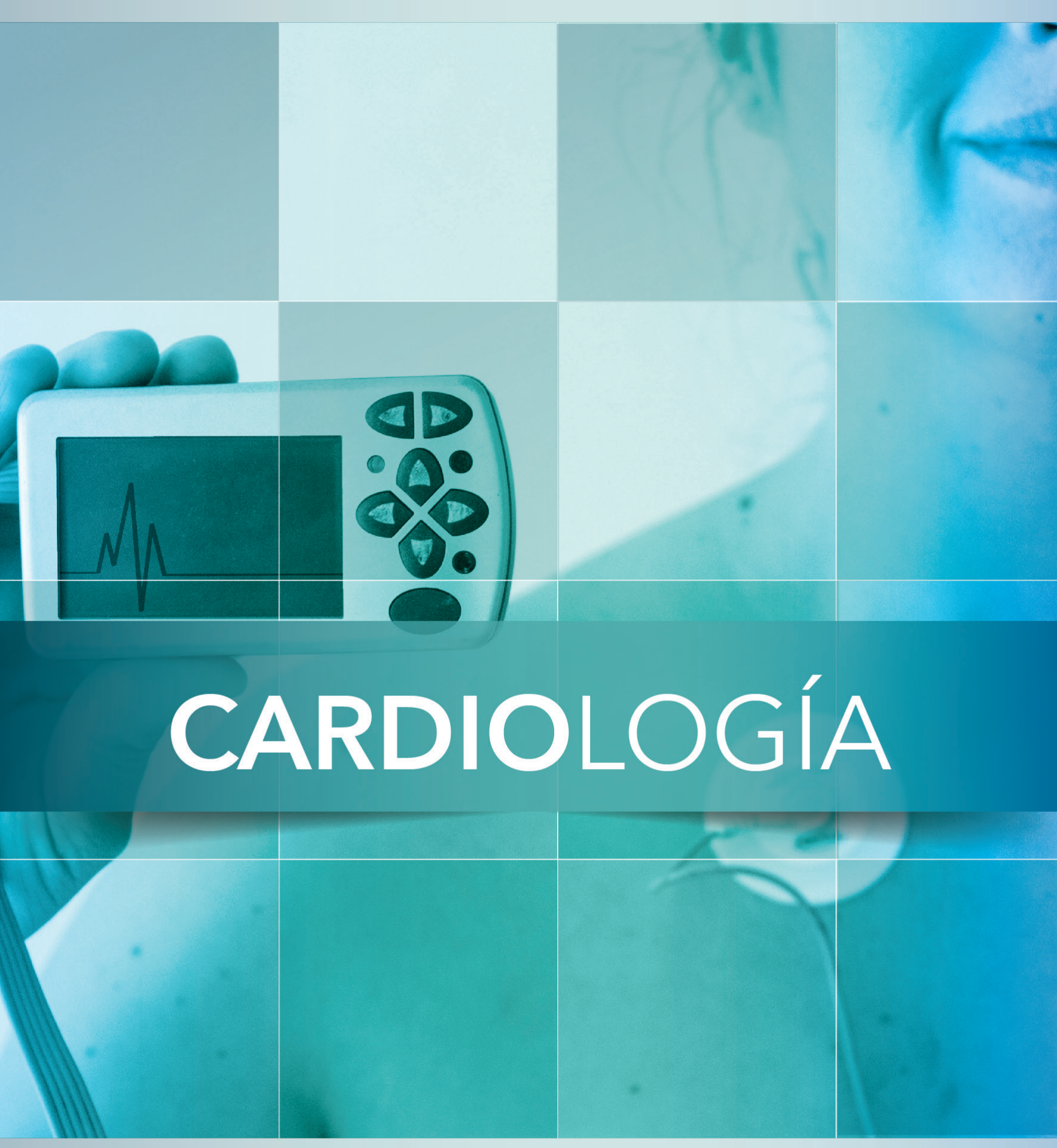


CÁculos $Y$

PROHÓSTICAS

EN MEDICINA INTERNA 


\section{INSUFICIENCIA CARDÍACA}

\section{CRITERIOS DE FRAMINGHAM}

\section{Utilidad}

Los criterios de Framingham son utilizados para el diagnóstico de insuficiencia cardíaca en función de criterios mayores y menores.

\section{Descripción}

- La insuficiencia cardíaca es un diagnóstico clínico. Los criterios de Framingham ofrecen un conjunto aceptable de parámetros necesarios para realizar el diagnóstico.

- Seleccione los criterios menores solo si no pueden explicarse por otra comorbilidad, por ejemplo, disnea en el ejercicio secundario a la enfermedad pulmonar obstructiva crónica.

\section{Parámetros}

\begin{tabular}{|c|c|}
\hline MAYORES & MENORES \\
\hline Disnea paroxística nocturna & Edema de miembros inferiores \\
\hline Ortopnea & Tos nocturna \\
\hline Ingurgitación yugular & Disnea de esfuerzo \\
\hline Estertores crepitantes pulmonares & Hepatomegalia \\
\hline Cardiomegalia & Derrame pleural \\
\hline Edema agudo de pulmón & Taquicardia $\geq 120$ LPM \\
\hline Galope ventricular (S3) & Disminución de la capacidad vital \\
\hline Reflujo hepatoyugular & al $1 / 3$ de su valor normal. \\
\hline Presión venosa $>16 \mathrm{~cm}$ de agua & \\
\hline $\begin{array}{l}\text { Pérdida de peso }>4,5 \mathrm{~kg} \text { en } 5 \text { días } \\
\text { en respuesta al tratamiento. }\end{array}$ & \\
\hline
\end{tabular}




\section{Interpretación}

\section{Diagnóstico de Insuficiencia cardíaca}

2 criterios mayores

1 criterio mayor y 2 criterios menores

\section{Bibliografía}

\section{Original/Primaria}

1. McKee PA, Castelli WP, McNamara PM, Kannel WB. La historia natural de la insuficiencia cardíaca congestiva: el estudio de Framingham. N Engl J Med [Internet]. 1971, 23 de diciembre [citado marzo 2019]; 285 (26): 1441-6. Disponible en: https: / / www.nejm.org/ doi / full/10.1056/ NEJM197112232852601. 


\section{Utilidad}

Propuesta por la NYHA (New York Heart Association), para estratificar la severidad de la insuficiencia cardíaca en función de la limitación al ejercicio físico.

\section{Descripción}

Es un sistema de aproximación clínica, que deriva en gran parte de la inferencia de la historia médica, donde se reúne la observación del paciente en determinadas formas de actividad física y ocasionalmente de medidas directas o indirectas de la función cardíaca en respuesta a ejercicios estandarizados.

\section{Parámetros}

\begin{tabular}{|c|l|}
\hline CLASE & \multicolumn{1}{|c|}{ CARACTERÍSTICAS } \\
\hline I & $\begin{array}{l}\text { Sin limitación de la actividad física; La actividad física } \\
\text { normal no causa fatiga excesiva, palpitaciones ni disnea. }\end{array}$ \\
\hline II & $\begin{array}{l}\text { Ligera limitación de la actividad física; cómodo en reposo; } \\
\text { la actividad física ordinaria produce fatiga, palpitaciones o } \\
\text { disnea. }\end{array}$ \\
\hline III & $\begin{array}{l}\text { Marcada limitación de la actividad física; cómodo en } \\
\text { reposo; una actividad menor a la normal causa fatiga, } \\
\text { palpitaciones o disnea. }\end{array}$ \\
\hline IV & $\begin{array}{l}\text { Incapaz de realizar cualquier actividad física sin } \\
\text { incomodidad; síntomas de insuficiencia cardíaca en } \\
\text { reposo; si se realiza alguna actividad física, aumenta la } \\
\text { incomodidad. }\end{array}$ \\
\hline
\end{tabular}




\section{Interpretación}

\begin{tabular}{|c|l|}
\hline \multicolumn{2}{|l|}{} \\
\hline CLASE I & Síntomas a los grandes esfuerzos \\
\hline CLASE II & Síntomas a los esfuerzos habituales \\
\hline CLASE III & Síntomas a los pequeños esfuerzos \\
\hline CLASE IV & Síntomas presentes incluso en reposo \\
\hline
\end{tabular}

\section{Bibliografía}

\section{Original/Primaria}

1. Dolgin M, Association NYH, Fox AC, Gorlin R, Levin RI, New York Heart Association. Comité de Criterios. Nomenclatura y criterios para el diagnóstico de enfermedades del corazón y grandes vasos. $9^{\mathrm{a}}$ ed. Boston, MA: Lippincott Williams y Wilkins; 1 de marzo de 1994.

\section{Validación}

1. Goldman L, Hashimoto B, Cook EF, Loscalzo A. Reproducibilidad comparativa y validez de los sistemas para evaluar la clase funcional cardiovascular: ventajas de una nueva escala de actividad específica. Circulation [Internet]. 1981 [citado marzo 2019];64(6):1227-34. Disponible en: https: / / www.ncbi.nlm.nih.gov/ pubmed/7296795/.

2. Raphael C, Briscoe C, Davies J, Whinnett ZI, Manisty C, Sutton R, et al. Limitaciones del sistema de clasificación funcional de la New York Heart Association y las distancias para caminar autoinformadas en la insuficiencia cardíaca crónica. Heart [Internet]. 2007 abril [citado marzo 2019]; 93(4):476-482. Disponible en: https://www.ncbi.nlm. nih.gov/pmc/articles/PMC1861501/. 


\section{ESTADIFICACIÓN ACC / AHA PARA LA INSUFICIENCIA CARDÍACA}

\section{Utilidad}

Describe las etapas de la insuficiencia cardíaca y proporciona recomendaciones terapéuticas en base a cada una de ellas.

\section{Descripción}

- Desarrollado conjuntamente por la ACC (American College Of Cardiology) y la AHA (American Heart Association) para complementar, pero no reemplazar, la clasificación funcional más ampliamente utilizada de la NYHA (New York Hearth Association).

- Hace coincidir la etapa con las opciones de tratamiento, a diferencia de otros sistemas de clasificación.

\section{Parámetros}

\begin{tabular}{|c|l|}
\hline ESTADIO & \multicolumn{1}{|c|}{ CARACTERÍSTICAS } \\
\hline A & $\begin{array}{l}\text { Con alto riesgo de insuficiencia cardíaca. Anomalía } \\
\text { estructural o funcional no identificada; sin signos ni } \\
\text { síntomas. }\end{array}$ \\
\hline B & $\begin{array}{l}\text { Enfermedad cardíaca estructural desarrollada claramente } \\
\text { en relación con insuficiencia cardíaca, pero sin signos ni } \\
\text { síntomas. }\end{array}$ \\
\hline C & $\begin{array}{l}\text { Insuficiencia cardíaca sintomática asociada a enfermedad } \\
\text { estructural subyacente. }\end{array}$ \\
\hline D & $\begin{array}{l}\text { Enfermedad cardíaca estructural avanzada y síntomas } \\
\text { acusados de insuficiencia cardíaca en reposo a pesar de } \\
\text { tratamiento médico máximo. }\end{array}$ \\
\hline
\end{tabular}




\section{Interpretación}

\begin{tabular}{|l|l|}
\hline & CONSIDERACIONES DE LA TERAPIA \\
\hline A & $\begin{array}{l}\text { Tratar la hipertensión } \\
\text { Fomentar el abandono del hábito de fumar } \\
\text { Tratar los trastornos de los lípidos } \\
\text { Fomentar el ejercicio regular } \\
\text { Desalentar el consumo de alcohol / uso de drogas ilícitas } \\
\text { Inhibidores de la ECA en pacientes apropiados }\end{array}$ \\
\hline B & $\begin{array}{l}\text { Todas las medidas en el estadio A } \\
\text { Inhibidores de la ECA en pacientes apropiados } \\
\text { Betabloqueantes en pacientes apropiados }\end{array}$ \\
\hline C & $\begin{array}{l}\text { Todas las medidas en la etapa A } \\
\text { Diuréticos, inhibidores de la ECA, bloqueadores beta o } \\
\text { digitálico para uso de rutina } \\
\text { Restricción de sal en la dieta }\end{array}$ \\
\hline D & $\begin{array}{l}\text { Todas las medidas en las etapas A, B y C } \\
\text { Dispositivos de asistencia mecánica } \\
\text { Infusiones inotrópicas IV continuas para paliación } \\
\text { Atención de hospicio (cuidados paliativos) }\end{array}$ \\
\hline
\end{tabular}

ECA: enzima convertidora de angiotensina.

\section{Bibliografía}

\section{Original/Primaria}

1. Hunt SA, Baker DW et al. Directrices de ACC / AHA para la evaluación y el tratamiento de la insuficiencia cardíaca crónica en el adulto: 
Resumen ejecutivo Un informe del Grupo de Trabajo sobre Prácticas de la American College of Cardiology / American Heart Association (Comité para revisar las Directrices de 1995 para la evaluación y el manejo del corazón Fallo): desarrollado en colaboración con la Sociedad Internacional para el trasplante de corazón y pulmón; Avalado por la Heart Failure Society of America. Circulation [Internet]. 11 de diciembre de 2001[citado 30 marzo 2019]; 104 (24): 2996-3007. Disponible en: https://www.ahajournals.org/doi/full/10.1161/ hc4901.102568. 


\section{SÍNDROME CORONARIO AGUDO}

\section{CLASIFICACIÓN DE KILLIP-KIMBALL}

\section{Utilidad}

Es una estratificación, que permite establecer un pronóstico de la evolución después de un Síndrome Coronario Agudo (SCA) y además predice la mortalidad en los primeros 30 días.

\section{Descripción}

- Puede ser utilizado tanto en STEMI (Infarto de miocardio con elevación del segmento ST), como en NSTEMI (Infarto de miocardio sin elevación del segmento ST).

\section{Parámetros}

\begin{tabular}{|c|l|}
\hline CLASE & \multicolumn{1}{c|}{ CARACTERÍSTICAS } \\
\hline I & $\begin{array}{l}\text { Auscultación cardíaca y pulmonar normales. } \\
\text { Presión arterial normal. }\end{array}$ \\
\hline \multirow{2}{*}{ II } & $\begin{array}{l}\text { 3er tono / Crepitantes bibasales } \\
\text { Presión arterial normal. }\end{array}$ \\
\hline III & $\begin{array}{l}\text { 3er tono / Edema agudo del pulmón } \\
\text { Presión arterial normal o baja. }\end{array}$ \\
\hline IV & Shock cardiogénico. \\
\hline
\end{tabular}

\section{Interpretación}

\begin{tabular}{|c|c|c|}
\hline CLASE & Mortalidad a 30 dias & Mortalidad hospitalaria \\
\cline { 1 - 2 } I & $2-3 \%$ & $<6 \%$ \\
\cline { 1 - 2 } II & $5-12 \%$ & $<17 \%$ \\
\cline { 1 - 2 } III & \multirow{2}{*}{$10-20 \%$} & $38 \%$ \\
\cline { 1 - 1 } IV & & $81 \%$ \\
\hline
\end{tabular}




\section{Bibliografía}

\section{Original/Primaria}

1. Killip T, Kimball JT. Tratamiento del infarto de miocardio en una unidad de cuidados coronarios. Una experiencia de dos años con 250 pacientes. Am J Cardiol [Internet]. 1967 [citado marzo 2019]; 20 (4): 457-64. Disponible en: https://www.ajconline.org/ article/0002-9149(67)90023-9/pdf.

\section{Validación}

1. Khot UN, Jia G, Moliterno DJ, , Lincoff AM, Khot MB, Harrington RA, et al. Importancia pronóstica del examen físico para la insuficiencia cardíaca en los síndromes coronarios agudos sin elevación del segmento ST: el valor perdurable de la clasificación de Killip. JAMA [Internet] 2003 [citado marzo 2019]; 290 (16): 2174-81. Disponible en: https: / / www.ncbi.nlm.nih.gov/pubmed/14570953.

2. DeGeare VS, Boura JA, Grines LL, O'Neill WW, Grines CL. Valor predictivo de la clasificación de Killip en pacientes sometidos a intervención coronaria percutánea primaria por infarto agudo de miocardio. Am J Cardiol [Internet]. 20011 de mayo [citado marzo 2019]; 87 (9): 1035-8. Disponible en: https: / / www.ncbi.nlm.nih.gov/ pubmed / 11348598 .

3. Nesković AN, Otasević P, Bojić M, Popović AD. Asociación de Killip en el ingreso y dilatación del ventrículo izquierdo después de un infarto de miocardio: una mirada más cercana a una antigua clasificación clínica. Am Heart J [Internet]. Febrero 1999 [citado marzo 2019]; 137 (2): 3617. Disponible en : https: / / www.ncbi.nlm.nih.gov/pubmed/ 9924172. 


\section{ÍNDICE DE RIESGO TIMI}

\section{Utilidad}

Proporciona una estimación de la mortalidad en pacientes con SCA, tomando en cuenta presión arterial, frecuencia cardíaca y edad.

\section{Descripción}

- Predice tanto la mortalidad hospitaria a corto como a largo plazo.

- Las estimaciones de mortalidad pueden ser útiles para determinar el nivel de atención y la intensidad del tratamiento.

\section{Fórmula}

Índice de riesgo TIMI $=\frac{\text { Frecuencia cardíaca } x(\text { Edad } / 10)^{2}}{\text { Presión arterial sistólica }}$

\section{Interpretación}

\begin{tabular}{|cl|}
\hline RESULTADO & \multicolumn{1}{c|}{ RIESGO/MORTALIDAD } \\
\hline \multirow{2}{*}{$\mathbf{1 - 2 8}$} & $\begin{array}{l}\text { TRI bajo, denota una mortalidad hospitalaria de } \\
\text { aproximadamente el 10\% o menos. }\end{array}$ \\
\hline $\mathbf{2} 29$ & $\begin{array}{l}\text { TRI intermedio/alto, denota una mortalidad } \\
\text { hospitalaria }>10 \% .\end{array}$ \\
\hline
\end{tabular}

TRI: índice de riesgo de trombólisis en el infarto de miocardio.

\section{Bibliografía}

\section{Original/Primaria}

1. Wiviott SD, Morrow DA, Frederick PD, Antman EM, Braunwald E; Registro Nacional de Infarto de Miocardio. Aplicación del índice de riesgo de trombólisis en el infarto de miocardio en el infarto de miocardio sin elevación del segmento ST: evaluación de pacientes en el Registro Nacional de Infarto de Miocardio. J Am Coll Cardiol [Internet]. 200618 de abril [citado marzo 2019]; 47 (8): 1553-8. Disponible en: https: / / www.ncbi.nlm.nih.gov/pubmed/16630990. 


\section{PUNTAJE DE RIESGO TIMI PARA STEMI}

\section{Utilidad}

Se usa para estimar la mortalidad en pacientes con infarto de miocardio, que tengan elevación del segmento ST.

\section{Descripción}

- La puntuación de riesgo TIMI para STEMI (infarto de miocardio con elevación del segmento ST) puede ayudar a un equipo médico de cardiología y/o de cuidados intensivos a mantener un mejor criterio sobre el riesgo/beneficio de los medicamentos (como la anticoagulación) y los procedimientos invasivos de un paciente.

- Debe utilizarse en pacientes con STEMI diagnosticado, no para evaluar a pacientes con dolor torácico.

\section{Parámetros}

\begin{tabular}{|l|c|}
\hline CRITERIOS & PUNTAJE \\
\hline Edad & \\
$<65$ años & 0 \\
$65-74$ años & 2 \\
$\geq 75$ años & 3 \\
\hline Diabetes mellitus / HTA o Angina & 1 \\
\hline Presión arterial sistólica $<100 \mathrm{mmHg}$ & 3 \\
\hline Frecuencia cardíaca $>100$ LPM & 2 \\
\hline Killip-Kimbal II-IV & 2 \\
\hline Peso $<67$ kg & 1 \\
\hline Elevación ST anterior o BRI & 1 \\
\hline Tiempo de tratamiento $>4$ horas & 1 \\
\hline
\end{tabular}

HTA: hipertensión arterial, BRI: bloqueo de rama izquierda. 


\section{Interpretación}

\begin{tabular}{|c|c|}
\hline PUNTAJE & Mortalidad a los 30 dias \\
\hline $\mathbf{0}$ & $0.8 \%$ \\
\hline $\mathbf{1}$ & $1.6 \%$ \\
\hline $\mathbf{2}$ & $2.2 \%$ \\
\hline $\mathbf{3}$ & $4.4 \%$ \\
\hline $\mathbf{4}$ & $7.3 \%$ \\
\hline $\mathbf{5}$ & $12.4 \%$ \\
\hline $\mathbf{6}$ & $16.1 \%$ \\
\hline $\mathbf{7}$ & $23.4 \%$ \\
\hline $\mathbf{8}$ & $26.8 \%$ \\
\hline $\mathbf{9}$ & $35.9 \%$ \\
\hline
\end{tabular}

\section{Bibliografía}

\section{Original/Primaria}

1. David A. Morrow, et. Alabama. Puntuación de riesgo TIMI para el infarto de miocardio con elevación del ST: una puntuación clínica conveniente junto a la cama para la evaluación de riesgos en la presentación: Un NPA intravenoso para el tratamiento de la circulación de subestudio en el ensayo de infarto de miocardio temprano II. Circulation [Internet] 2000 [citado marzo 2019]; 102: 2031-2037. https: / / www.ncbi.nlm.nih.gov/pubmed/11044416.

\section{Validacion}

1. Morrow DA, Antman EM, Parsons L, de Lemos JA, de Cannon CP, Giugliano RP. Aplicación de la puntuación de riesgo TIMI para el IM con elevación del ST en el Registro Nacional de Infarto de Miocardio 3. JAMA [Internet]. Septiembre 2001 [citado marzo 2019]; 286 (11): 13569. Disponible en: https: / www.ncbi.nlm.nih.gov/ pubmed/11560541

2. Bradshaw PJ, Ko DT, Newman AM, Donovan LR. Validación del índice de riesgo de trombólisis en el infarto de miocardio (TIMI, por sus siglas en inglés) para predecir la mortalidad temprana en una cohorte poblacional de pacientes STEMI y no STEMI. Can J Cardiol 
[Internet]. Enero 2007 [citado marzo 2019] ;23(1):51-6. Disponible en: https: / / www.ncbi.nlm.nih.gov/pubmed/17245483.

3. Souto D, Pederneiras C, Hatschbach L, Frnandes EU. Validación de escala TIMI para STEMI. International Journal of Cardiovascular Sciences [Internet]. 2016 [citado marzo 2019];29(3):189-197. Disponible en: http:/ / www.onlineijcs.org/sumario/29/pdf/en_v29n3a06.pdf 


\section{PUNTAJE DE RIESGO TIMI PARA ANGINA INESTABLE E INFARTO DE MIOCARDIO SIN ELEVACIÓN DE SEGMENTO ST}

\section{Utilidad}

Se usa para estimar la mortalidad en pacientes con angina inestable y sin elevación del segmento ST.

\section{Descripción}

- Puede usarse para ayudar a estratificar el riesgo de pacientes con presunto dolor torácico de tipo isquémico.

- Una puntuación de riesgo TIMI de 0 o 1 no es igual a cero riesgo de resultados adversos.

\section{Parámetros}

\begin{tabular}{|l|c|}
\hline CRITERIOS & PUNTAJE \\
\hline Edad $\geq 65$ años. & 1 \\
\hline Factores de riesgo cardiovascular tradicionales $\geq 3$. & 1 \\
\hline Antecedente de estenosis coronaria mayor o igual a 50\%. & 1 \\
\hline Uso de ASA en los últimos 7 días. & 1 \\
\hline $\begin{array}{l}\text { Dos o más episodios de angina en las 24 horas previas al } \\
\text { ingreso. }\end{array}$ & 1 \\
\hline $\begin{array}{l}\text { Desviación del segmento ST en el electrocardiograma } \\
\text { inicial. }\end{array}$ & 1 \\
\hline Elevación de biomarcadores de necrosis miocárdica. & 1 \\
\hline
\end{tabular}

\section{Interpretación}

\begin{tabular}{|c|l|}
\hline PUNTAJE & CATEGORIZACIÓN \\
\hline $0-2$ & Bajo riesgo \\
\hline $3-4$ & Riesgo intermedio \\
\hline $5-7$ & Alto riesgo \\
\hline
\end{tabular}




\section{Bibliografía}

\section{Original/Primaria}

1. Antman EM, Cohen M, Bernink PJLM, McCabe $\mathrm{CH}$, Hoacek T, Papuchis G, et al. La puntuación de riesgo TIMI para angina inestable / IM sin elevación del ST: un método de pronóstico y terapéutica toma de decisiones JAMA [Internet]. 2000 [citado marzo 2019]; 284 (7): 835-42. Disponible en: https:/ / www.mdcalc.com/timi-risk-score-uanstemi\# evidence.

\section{Validación}

1. Pollack CV, Sitios FD, Shofer FS, Sease KL, Hollander JE. Aplicación de la puntuación de riesgo TIMI para la angina inestable y el síndrome coronario agudo sin elevación del ST en una población de dolor de tórax en el departamento de emergencia no seleccionado. Acad Emerg Med [Internet]. 2006 [citado marzo 2019]; 13(1):13-18. Disponible en: http: / / www.cardiocast.net/ohds/TIMI_Score.pdf.

2. Scirica BM, Cannon CP, Antman EM, Murphy SA, Morrow DA, Sabatine MS, et al. Validación de la trombolisis en el infarto de miocardio (TIMI) puntuación de riesgo para angina de pecho inestable y miocardio sin elevación del segmento ST Infarto en el registro TIMI III. Am J Cardiol [Internet]. 20021 de agosto [citado marzo 2019]; 90 (3): 303-5. Disponible en: https: / / www.ncbi.nlm.nih.gov/ pubmed/12127617.

3. Chase M, Robey JL, Zogby KE, Sease KL, Shofer FS, Hollander JE. Validación prospectiva de la Trombolisis en la puntuación de riesgo de infarto de miocardio en la población de urgencias con dolor torácico. Ann Emerg Med [Internet]. Septiembre de 2006 [citado marzo 2019];48(3):252-9. Disponible en: https://www.ncbi.nlm.nih. gov/pubmed / 16934646 . 


\section{CLASIFICACIÓN HEMODINÁMICA DE FORRESTER}

\section{Utilidad}

Evalúa la insuficiencia cardíaca y la mortalidad en el contexto de infarto agudo de miocardio. Los valores de la Presión Capilar Pulmonar (PCP) e Índice Cardíaco (IC) son medidos con cateterismo cardíaco.

\section{Descripción}

- Es equivalente a la clasificación de Killip y Kimball, excepto en el estadío III.

- Estas clasificaciones son importantes tanto desde el punto de vista del manejo como del pronóstico de los pacientes.

\section{Parámetros}

\begin{tabular}{|c|c|c|c|c|}
\hline GRADO & $\begin{array}{c}\text { IC } \\
\left(\mathbf{L} / \mathbf{m i n} / \mathbf{m}^{2}\right. \\
\mathbf{s c})\end{array}$ & $\begin{array}{c}\text { PCP } \\
(\mathbf{m m H g})\end{array}$ & CLÍNICA & $\begin{array}{c}\text { MORTALIDAD } \\
\mathbf{( \% )}\end{array}$ \\
\hline I & $>2.2$ & $<18$ & $\begin{array}{c}\text { Sin insuficiencia } \\
\text { cardíaca }\end{array}$ & 3 \\
II & $>2.2$ & $>18$ & $\begin{array}{c}\text { Congestión } \\
\text { pulmonar }\end{array}$ & 10 \\
III & $<2.2$ & $<18$ & Hipovolemia & $15-30$ \\
IV & $<2.2$ & $>18$ & Shock & $80-90$ \\
\hline
\end{tabular}

IC: índice cardíaco, PCP: presión capilar pulmomar.

Interpretación

\begin{tabular}{|c|l|c|}
\hline \multicolumn{3}{|c|}{ CATEGORIZACIÓN } \\
\hline I & $\begin{array}{l}\text { Sin congestión pulmonar con índice } \\
\text { cardíaco adecuado. }\end{array}$ & $3 \%$ \\
\hline II & $\begin{array}{l}\text { Congestión pulmonar con índice cardíaco } \\
\text { adecuado. }\end{array}$ & $10 \%$ \\
\hline
\end{tabular}




\begin{tabular}{|c|l|c|}
\hline III & $\begin{array}{l}\text { Índice cardíaco bajo, sin congestión } \\
\text { pulmonar. }\end{array}$ & $15-30 \%$ \\
\hline IV & $\begin{array}{l}\text { Congestión pulmonar e índice cardíaco } \\
\text { bajo. }\end{array}$ & $80-90 \%$ \\
\hline
\end{tabular}

\section{Bibliografía}

\section{Original/Primaria}

Forrester JS, Diamond GA, Swan HJ. Clasificación correlativa de la función clínica y hemodinámica tras el infarto agudo de miocardio. Am J Cardiol [Internet]. Febrero 1977 [citado marzo 2019]; 39(2):137-45. Disponible en: https: / / www.ncbi.nlm.nih.gov/ pubmed/ 835473. 


\section{GRACE SCORE 2.0}

\section{Utilidad}

La escala GRACE (Global Registry of Acute Coronary Events), es un modelo aplicado a todos los síndromes coronarios agudos, que estima el riesgo de muerte hospitalaria y a los seis meses.

\section{Descripción}

- El GRACE Score es un sistema de puntuación estudiado prospectivamente para estimar la mortalidad hospitalaria y de 6 meses a 3 años.

\section{Parámetros}

\begin{tabular}{|l|l|l|c|}
\hline CRITERIOS & PUNTOS & CRITERIOS & PUNTOS \\
\hline \multicolumn{2}{|l|}{ Edad } & Frecuencia cardíaca & 0 \\
\hline$<40$ años & 0 & $<70$ & 7 \\
$40-49$ años & 18 & $70-89$ & 13 \\
$50-59$ años & 36 & $90-109$ & 23 \\
$60-69$ años & 55 & $110-149$ & 36 \\
$70-79$ años & 73 & 150 & 46 \\
$\geq 80$ años & 91 & $\geq 200$ & 2 \\
\hline Presión arterial sistólica & Creatinina & 5 \\
\hline$<80$ & 63 & $0-0,39$ & 8 \\
$80-99$ & 58 & $0,4-0,79$ & 11 \\
$100-119$ & 47 & $0,8-1,19$ & 14 \\
$120-139$ & 37 & $1,20-1,59$ & 23 \\
$140-159$ & 26 & $1,60-1,99$ & 31 \\
$160-199$ & 11 & $2-3,90$ & $\geq 4$ \\
$\geq 200$ & 0 & &
\end{tabular}




\begin{tabular}{|l|l|l|c|}
\hline \multicolumn{2}{|l|}{ Killip-Kimball } & \multicolumn{2}{|l|}{ Otros factores de riesgo } \\
\hline Clase I & 0 & Cambios del segmento ST & 30 \\
\cline { 2 - 4 } Clase II & 21 & $\begin{array}{l}\text { Biomarcadores de necrosis } \\
\text { cardíaca }\end{array}$ & 15 \\
\cline { 2 - 4 } Clase III & 43 & Paro cardíaco al ingreso & 43 \\
Clase IV & 64 & & \\
\hline
\end{tabular}

\section{Interpretación}

\begin{tabular}{|c|c|}
\hline RANGO DE PUNTUACIÓN & RIESGO DE MORTALIDAD \\
\hline $\mathbf{0 - 8 7}$ & $0-2 \%$ \\
\hline $\mathbf{8 8 - 1 2 8}$ & $3-10 \%$ \\
\hline $\mathbf{1 2 9 - 1 4 9}$ & $10-20 \%$ \\
\hline $\mathbf{1 5 0 - 1 7 3}$ & $20-30 \%$ \\
\hline $\mathbf{1 7 4 - 1 8 2}$ & $40 \%$ \\
\hline $\mathbf{1 8 3 - 1 9 0}$ & $50 \%$ \\
\hline $\mathbf{1 9 1 - 1 9 9}$ & $60 \%$ \\
\hline $\mathbf{2 0 0 - 2 0 7}$ & $70 \%$ \\
\hline $\mathbf{2 0 8 - 2 1 8}$ & $80 \%$ \\
\hline $\mathbf{2 1 9 - 2 8 4}$ & $90 \%$ \\
\hline $\mathbf{2 8 5}$ & $99 \%$ \\
\hline
\end{tabular}

En el contexto de síndrome coronario agudo sin elevación del ST:

Score de GRACE y Mortalidad Intrahospitalaria.

\begin{tabular}{l|l|l} 
Categoría de Riesgo & \multicolumn{1}{l}{ Score GRACE } & Mortalidad (\%) \\
\hline Bajo riesgo & $1-108$ & $1-3$ \\
\hline Riesgo intermedio & $109-140$ & Mayor a 3 \\
\hline Alto riesgo & $141-372$ & \\
\hline
\end{tabular}

Score de GRACE y Mortalidad del Alta Hospitalaria a los 6 meses. 


\begin{tabular}{l|l|l} 
Categoría de Riesgo & \multicolumn{1}{l}{ Score GRACE } & Mortalidad (\%) \\
\hline Bajo riesgo & $1-88$ & Menor de 3 \\
\hline Riesgo intermedio & $89-118$ & $3-8$ \\
\hline Alto riesgo & $119-263$ & Mayor de 8 \\
\hline
\end{tabular}

En el contexto de síndrome coronario agudo con elevación del ST:

Score de GRACE y Mortalidad Intrahospitalaria.

\begin{tabular}{l|l|l} 
Categoría de Riesgo & \multicolumn{1}{l}{ Score GRACE } & \multicolumn{1}{l}{ Mortalidad (\%) } \\
\hline Bajo riesgo & $49-125$ & Menor de 2 \\
\hline Riesgo intermedio & $126-154$ & $2-5$ \\
\hline Alto riesgo & $155-319$ & Mayor de 5 \\
\hline
\end{tabular}

Score de GRACE y Mortalidad del Alta hospitalaria a los 6 meses.

\begin{tabular}{l|l|l} 
Categoría de Riesgo & \multicolumn{1}{l}{ Score GRACE } & Mortalidad (\%) \\
\hline Bajo riesgo & $27-99$ & Menor de 4.4 \\
\hline Riesgo intermedio & $100-127$ & $4.4-11$ \\
\hline Alto riesgo & $128-263$ & Mayor de 11 \\
\hline
\end{tabular}

\section{Bibliografía}

\section{Original/Primaria}

1. Fox KA, Dabbous OH, Goldberg RJ, Pieper KS, Eagle KA, Van de Werf F,et al. Predicción del riesgo de muerte e infarto de miocardio en los seis meses posteriores a la presentación con síndrome coronario agudo: estudio observacional multinacional prospectivo (GRACE). BMJ [Internet]. 25 de noviembre de 2006 [citado marzo 2019]; 333 (7578): 1091. Disponible en: https://www.ncbi.nlm.nih.gov/ pubmed/17032691.

\section{Validación}

1. Elbarouni B, Goodman SG, Yan RT, Welsh RC, Kornder JM, Deyoung JP, et al. Validación de la puntuación de riesgo del Evento Coronario Agudo (GRACE) para la mortalidad hospitalaria en pacientes con síndrome coronario agudo en Canadá. Am Heart J [Internet]. 2009 septiembre [citado marzo 2019]; 158(3): 392-9. Disponible en: https: / / www.ncbi.nlm.nih.gov/pubmed/19699862. 


\section{INTERVALO QT CORREGIDO}

\section{Utilidad}

Corrige el intervalo QT para los extremos de la frecuencia cardíaca. Parámetros

Frecuencia cardíaca Intervalo QT
60-100 latidos/ $\mathrm{min}$ msec.

Fórmula

$$
Q T \text { corregido }=\frac{\text { Intervalo } Q T}{\sqrt{\text { Intervalo } R R}}
$$

\section{Interpretación}

\section{Normal}

Un QT más largo pone al paciente en mayor riesgo de torsade de pointes.

\section{CAUSAS DE QT PROLONGADO}

\begin{tabular}{|ll|}
\hline & Causas cardíacas intrínsecas \\
Anomalías electrolíticas & Isquemia miocárdica. \\
Hipocalcemia & Después de un paro cardíaco. \\
Hipopotasemia & Cetoacidosis diabética. \\
Hipomagnesemia & Miocardiopatía. \\
& Bradicardia severa, bloqueo AV de \\
& alto grado. \\
& Síndrome de QT largo congénito. \\
\hline
\end{tabular}




\section{Causas centrales}

Presión intracraneal elevada

\section{Medicamentos}

Disfunción autonómica

Antiarrítmicos.

Hipotiroidismo

Drogas psicotrópicas.

Hipotermia

Otras drogas.

\section{Bibliografía}

\section{Original/Primaria}

1. Bazett HC. An analysis of the time-relations of electrocardiograms. Heart 1920 [citado marzo 2019];(7):353-37.

\section{Validación}

1. Lesson III. Characteristics of the Normal ECG Frank G. Yanowitz, MD. Professor of Medicine. University of Utah School of Medicine. 


\section{FIBRILACIÓN AURICULAR}

\section{SCORE CHA2DS2-VASC}

\section{Utilidad}

Se aplica para determinar anticoagulación, en pacientes con fibrilación auricular, para evitar fenómenos cardioembólicos.

\section{Descripción}

- La puntuación CHA2DS2-VASc (Birmingham 2009) se desarrolló después de identificar factores adicionales de riesgo de accidente cerebrovascular en pacientes con fibrilación auricular. El estudio de validación incluyó a 1.084 pacientes con FA no valvular, no en anticoagulación, mayores de 18 años con EKG o diagnóstico de FA Holter en entornos ambulatorios y hospitalarios de 182 hospitales en 35 países, entre 2003 y 2004; habían conocido el estado tromboembólico al año base de datos de Euro Heart Survey. El punto final utilizado fue accidente cerebrovascular $\mathrm{u}$ otro evento tromboembólico. El estudio mostró que a medida que aumentaba el puntaje CHA2DS2-VASc, también aumentaba la tasa de eventos tromboembólicos dentro de 1 año en pacientes no anticoagulados con FA no valvular.

- Se consideró que un puntaje de 0 era de bajo riesgo para eventos TE (ninguno visto en la cohorte al año), un puntaje de 1 riesgo intermedio (tasa de $0,6 \%$ a 1 año) y un riesgo mayor a 1 (tasa de $3 \%$ a 1 año).

Parámetros

\begin{tabular}{|l|c|}
\hline Score CHA2DS2-Vasc. & Puntaje \\
\hline $\begin{array}{l}\text { C: Insuficiencia cardíaca congestiva o disfunción } \\
\text { ventricular izquierda. }\end{array}$ & 1 \\
\hline H: Hipertensión Arterial. & 1 \\
\hline A: Edad (mayor o igual a 75 años). & $1(2)$ \\
\hline D: Diabetes Mellitus. & 1 \\
\hline S: Stroke. & 2 \\
\hline Vasc: Enfermedad vascular periférica. & 1 \\
\hline Categoría de sexo: Femenino. & 1 \\
\hline
\end{tabular}




\section{Interpretación}

CHA2DS2-VASC $\geq 1$ en Hombre: Anticoagulación.

CHA2DS2-VASC $\geq 2$ en Mujeres: Anticoagulación.

\begin{tabular}{|c|c|c|}
\hline Score $\mathrm{CHA}_{2} \mathrm{DS}_{2}$-VASc & $\begin{array}{c}\text { Riesgo de Stroke } \\
\text { Isquémico }\end{array}$ & $\begin{array}{c}\text { Riesgo de stroke/TIA/ } \\
\text { embolismo sistémico }\end{array}$ \\
\hline $\mathbf{0}$ & $0.2 \%$ & $0.3 \%$ \\
\hline $\mathbf{1}$ & $0.6 \%$ & $0.9 \%$ \\
\hline $\mathbf{2}$ & $2.2 \%$ & $2.9 \%$ \\
\hline $\mathbf{3}$ & $3.2 \%$ & $4.6 \%$ \\
\hline $\mathbf{4}$ & $4.8 \%$ & $6.7 \%$ \\
\hline $\mathbf{5}$ & $7.2 \%$ & $10.0 \%$ \\
\hline $\mathbf{6}$ & $9.7 \%$ & $13.6 \%$ \\
\hline $\mathbf{7}$ & $11.2 \%$ & $15.7 \%$ \\
\hline $\mathbf{8}$ & $10.8 \%$ & $15.2 \%$ \\
\hline $\mathbf{9}$ & $12.2 \%$ & $17.4 \%$ \\
\hline
\end{tabular}

TIA: accidente isquémico transitorio.

\section{Bibliografía}

\section{Original/Primaria}

1. Lip GY, Nieuwlaat R, Pisters R, Lane DA, Crijns HJ. Refining clinical risk stratification for predicting stroke and thromboembolism in atrial fibrillation using a novel risk factor-based approach: the euro heart survey on atrial fibrillation. Chest. 2010 Feb;137(2):263-72. doi: 10.1378/ chest.09-1584. Epub 2009 Sep 17. PubMed PMID: 19762550.

\section{Validación}

1. Ntaios G, et al. CHADS2, CHA2DS2-VASc, and long-term stroke outcome in patients without atrial fibrillation. March 12, 2013 80:10091017; published ahead of print February 13, 2013.

2. Friberg L, Rosenqvist M, Lip GY. Evaluation of risk stratification schemes for ischaemic stroke and bleeding in 182678 patients with atrial fibrillation: the Swedish Atrial Fibrillation cohort study. Eur 
Heart J. 2012 Jun;33(12):1500-10. doi: 10.1093/eurheartj/ ehr488. Epub 2012 Jan 13. PubMed PMID: 22246443.

3. Okumura $\mathrm{K}$, Inoue $\mathrm{H}$, Atarashi $\mathrm{H}$, Yamashita $\mathrm{T}$, Tomita $\mathrm{H}$, Origasa $\mathrm{H}$; J-RHYTHM Registry Investigators.Validation of $\mathrm{CHA}_{2} \mathrm{DS}_{2}-$ VASc and HAS-BLED scores in Japanese patients with nonvalvular atrial fibrillation: an analysis of the J-RHYTHM Registry. Circ J. 2014;78(7):1593-9. Epub 2014 Apr 22. 


\section{SCORE HAS-BLED}

\section{Utilidad}

Se aplica para determinar el riesgo de sangrado en un paciente con fibrilación auricular y que requiere anticoagulación. Considere usar el puntaje HAS-BLED en lugar de, o en conjunto con, otros puntajes de riesgo de sangrado como HEMORR2HAGES y ATRIA, para determinar el riesgo de sangrado mayor en un paciente con fibrilación auricular. Considere comparar el riesgo de hemorragia mayor según lo calculado por el puntaje HAS-BLED, con el riesgo de eventos tromboembólicos de CHA2DS2-VASc para determinar si el beneficio de la anticoagulación supera el riesgo.

\section{Descripción}

- Los factores clínicos que contribuyen al riesgo de accidente cerebrovascular y apoyan la anticoagulación en pacientes con fibrilación auricular, son con frecuencia factores de riesgo de sangrado también. El puntaje HAS-BLED se desarrolló como un puntaje de riesgo práctico para estimar el riesgo a 1 año de hemorragia mayor, en pacientes con fibrilación auricular. El estudio incluyó a 5.333 pacientes ambulatorios y hospitalizados con FA de hospitales académicos y no académicos en 35 países miembros de la Sociedad Europea de Cardiología.

\section{Parámetros}

\begin{tabular}{|l|l|}
\hline Score HAS-BLED & Puntaje \\
\hline H: Hipertensión arterial. & 1 \\
\hline A: Función renal/hepática anormal. & 1 \\
\hline S: Stroke. & 1 \\
\hline B: Historia de sangrado o predisposición. & 1 \\
\hline L: Labilidad del INR. & 1 \\
\hline E: Edad (mayor de 65 años). & 1 \\
\hline D: Drogas/alcohol concomitante. & 1 \\
\hline
\end{tabular}




\section{Interpretación}

Score HAS-BLED $\geq 3$ puntos: Precaución, mayor riesgo de sangrado y por lo tanto, mayor seguimiento para la corrección de factores modificables y minimizar el riesgo de sangrado. Un Score HAS-BLED $\geq 3$ por sí solo no contraindica anticoagulación.

\section{Bibliografía}

\section{Original/Primaria}

1. Pisters R, Lane DA, Nieuwlaat R, et al. A Novel User-Friendly Score (Has-Bled) To Assess 1-Year Risk Of Major Bleeding In Patients With Atrial Fibrillation: The Euro Heart Survey. Chest. 2010;138(5):10931100.

\section{Validación}

1. Lip GY, Frison L, Halperin JL, Lane DA. Comparative validation of a novel risk score for predicting bleeding risk in anticoagulated patients with atrial fibrillation: the HAS-BLED (Hypertension, Abnormal Renal/Liver Function, Stroke, Bleeding History or Predisposition, Labile INR, Elderly, Drugs / Alcohol Concomitantly) score. J Am Coll Cardiol. 2011 Jan 11;57(2):173-80. doi: 10.1016/j.jacc.2010.09.024. Epub 2010 Nov 24. 


\section{SCORE SAME-TT2R2}

\section{Utilidad}

Se aplica para elegir el tipo de anticoagulante (Antagonista de la vitamina $\mathrm{K}$ o nuevos anticoagulantes orales) en fibrilación auricular no valvular (estenosis mitral moderada a severa y prótesis valvular mecánica).

\section{Descripción}

La escala SAMe-TT2R2, atiende a numerosas variables clínicas: sexo femenino, edad $<60$ años, antecedentes médicos (más de 2 comorbilidades: hipertensión arterial, diabetes mellitus, enfermedad coronaria, vasculopatía periférica, insuficiencia cardíaca, ictus previo, enfermedad pulmonar crónica, insuficiencia renal o hepática), tratamiento (fármacos que puedan interferir con la anticoagulación), tabaquismo activo y raza. Los pacientes que presentan una puntuación con SAMe-TT2R2>2, tienen mayor probabilidad de tener un control pobre de la anticoagulación, estimado por medio del tiempo en rango terapéutico (TTR) $<65-70 \%$; en cambio, aquellos con una puntuación SAMe-TT2R2 de 0-1 tienen una mayor probabilidad de presentar un adecuado control de la anticoagulación.

\section{Parámetros}

\begin{tabular}{|l|l|}
\hline Score SAMeTT2R2 & Puntaje \\
\hline S: Sexo (mujer). & 1 \\
\hline A: Edad (menor de 60 años). & 1 \\
\hline $\begin{array}{l}\text { Me: Historia clínica (DM, IC, CI/IMA, Enf arterial } \\
\text { periférica, ACV previo, Enf renal, hepática o pulmonar). }\end{array}$ & 1 \\
\hline T: Tratamiento (amiodarona). & 1 \\
\hline T: Tabaco. & 2 \\
\hline R: Raza (no caucásico). & 2 \\
\hline
\end{tabular}

\section{Interpretación}

Score SAMeTT2R2: 0-1 punto: Es favorable el uso de Vit K Antagonistas: Warfarina 
Score SAMeTT2R2: >2 puntos: Es favorable el uso de nuevos anticoagulantes orales: Dabigatran, Rivaroxaban, Apixaban y Edoxaban.

\section{Bibliografía}

\section{Original/Primaria}

1. S. Apostolakis, R.M. Sullivan, B. Olshansky, G.Y. Lip. Factors affecting quality of anticoagulation control among patients with atrial fibrillation on warfarin: the SAMe-TT2R2 score. Chest., 144 (2013), pp. 1555-1563

\section{Validación}

1. Poli D, Antonucci E, Testa S, Lip GYH. A pros- pective validation of the SAME-TT2R 2 score: how to identify atrial fibrillation patients who will have good anticoagulation control on warfarin. Intern Emerg Med. 2014;9(4):443-7.

2. Gallego P, Roldán V, Marin F, Gálvez J, Valdés M, Vicente V, et al. SAMETTR score, time in therapeutic range and outcomes in anticoagulated pa- tients with atrial fibrillation. Am J Med 2014; 127(11):1083-8.

3. Abumuaileq RR-Y, Abu-Assi E, Raposei- ras-Roubin S, López-López A, Redondo-Dié- guez A, Álvarez-Iglesias D, et al. Evaluation of SAMe- TT2R2 risk score for predicting the quality of anticoagulation control in a real-world cohort of pa- tients with non-valvular atrial fibrillation on vita- min-K antagonists. Europace 2015;17(5):711-7. 

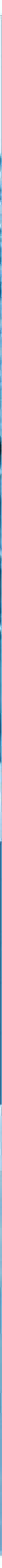
CÁculos $Y$

PROHÓSTICAS

EN MEDICINA INTERNA 


\section{NEUMONÍA ADQUIRIDA EN LA COMUNIDAD}

\section{ESCALA CURB-65}

\section{Utilidad}

Es una escala que predice la mortalidad de un paciente con Neumonía Adquirida en la Comunidad y además asocia la necesidad de tratamiento.

\section{Descripción}

- Es rápido de calcular, requiere la información probablemente disponible del paciente y proporciona una excelente estratificación del riesgo de mortalidad de la neumonía adquirida en la comunidad.

- Puede facilitar una mejor utilización de los recursos y la iniciación del tratamiento.

\section{Parámetros}

\begin{tabular}{|c|c|c|}
\hline \multicolumn{2}{|l|}{ CRITERIOS } & PUNTAJE \\
\hline $\begin{array}{c}\text { C } \\
\text { Confusion }\end{array}$ & Confusión & 1 \\
\hline $\begin{array}{c}\mathbf{U} \\
\text { Blood Urea } \\
\text { nitrogen }\end{array}$ & $\mathrm{BUN}>19 \mathrm{mg} / \mathrm{dl}$ & 1 \\
\hline $\begin{array}{c}\mathbf{R} \\
\text { Respiratory rate }\end{array}$ & Frecuencia respiratoria $\geq 30 \mathrm{LPM}$ & 1 \\
\hline $\begin{array}{c}\text { B } \\
\text { Blood pressure }\end{array}$ & $\begin{array}{c}\text { Presión arterial sistólica }<90 \mathrm{mmHg} \\
\mathrm{o} \\
\text { Presión arterial diastólica } \leq 60 \\
\mathrm{mmHg}\end{array}$ & 1 \\
\hline 65 & Edad $\geq 65$ años & 1 \\
\hline
\end{tabular}




\section{Interpretación}

\begin{tabular}{|c|c|l|}
\hline PUNTAJE & MORTALIDAD & \multicolumn{1}{|c|}{ RECOMENDACIÓN } \\
\hline $\mathbf{0}$ & $0.6 \%$ & $\begin{array}{l}\text { Bajo Riesgo. Considerar tratamiento } \\
\text { ambulatorio. }\end{array}$ \\
\hline $\mathbf{1}$ & $2.7 \%$ & $\begin{array}{l}\text { Bajo Riesgo. Considerar tratamiento } \\
\text { ambulatorio. }\end{array}$ \\
\hline $\mathbf{2}$ & $6.8 \%$ & $\begin{array}{l}\text { Riesgo intermedio, hospitalización } \\
\text { de corta estancia o tratamiento } \\
\text { ambulatorio con supervisión estricta. }\end{array}$ \\
\hline $\mathbf{3}$ & $14 \%$ & $\begin{array}{l}\text { Neumonía grave. Considerar } \\
\text { hospitalización. }\end{array}$ \\
\hline $\mathbf{4}$ & $27.8 \%$ & $\begin{array}{l}\text { Neumonía severa. Considerar } \\
\text { hospitalización. }\end{array}$ \\
\hline $\mathbf{5}$ & $57 \%$ & $\begin{array}{l}\text { Neumonía muy grave. Considerar } \\
\text { ingreso a UCI. }\end{array}$ \\
\hline
\end{tabular}

UCI: Unidad de Cuidados Intensivos.

\section{Bibliografía}

\section{Original/Primaria}

1. Lim W, van der Eerden MM, Laing R, Boersma W, Karalus N, Town $\mathrm{G}$, et. Al. Definición de la gravedad de la neumonía adquirida en la comunidad en la presentación al hospital: un estudio internacional de derivación y validación. Thorax [Internet]. 2003 mayo [citado marzo 2019];58(5):377-382. Disponible en: https: / / www.ncbi.nlm.nih.gov/ pmc/articles / PMC1746657 / ?tool=pmcentrez.

\section{Validación}

1. Shah BA, Ahmed W, Dhobi GN, Shah NN, Khusheed SQ, Haq I. Validez del índice de gravedad de neumonía y los sistemas de puntuación de severidad CURB-65 en neumonía adquirida en la comunidad en un entorno indígena. The Indian Journal of Chest Diseases \& Allied Sciences [Internet]. 2010 [citado 27 marzo 2019]; Vol.52.:10-17. Disponible en: http:/ / medind.nic.in/iae/t10/i1/iaet10i1p9.pdf.

2. Aujesky D1, Auble TE, Yealy DM, Stone RA, Obrosky DS, et.al. Comparación prospectiva de tres reglas de predicción validadas para 
el pronóstico en la neumonía adquirida en la comunidad. Am J Med [Internet]. 2005 abril [citado marzo 2019];118(4):384-92. Disponible en: https: / / www.ncbi.nlm.nih.gov/pubmed/15808136.

3. British Thoracic Society, Myint PK, Kamath AV, Vowler SL, Maisey DN, Harrison BD. Criterios de evaluación de la gravedad recomendados por la British Thoracic Society (BTS) para la neumonía adquirida en la comunidad (CAP) y pacientes mayores. ¿Se deben utilizar los criterios de SOAR (presión arterial sistólica, oxigenación, edad y frecuencia respiratoria) en las personas mayores? Un estudio compilatorio de dos cohortes prospectivos. Age Ageing [Internet]. 2006 mayo [citado marzo 2019]; 35(3):286-91. Disponible en: https: / / www.ncbi.nlm.nih. gov/pubmed / 16638769 . 


\section{ESCALA CRB-65}

\section{Utilidad}

Es una escala de evaluación muy similar al CURB-65, la única diferencia es que no toma en cuenta los niveles de urea sanguínea.

\section{Descripción}

- Al prescindir de estudios complementarios (urea) puede servir para el manejo en atención primaria de salud.

\section{Parámetros}

\begin{tabular}{|c|l|c|}
\hline \multicolumn{1}{|c|}{ CRITERIOS } & PUNTAJE \\
\hline $\begin{array}{c}\text { Confusion } \\
\text { R }\end{array}$ & Confusión & 1 \\
\hline Respiratory rate & Frecuencia respiratoria $\geq 30$ LPM & 1 \\
\hline B & $\begin{array}{l}\text { Presión arterial sistólica < } \\
\text { mmHg o } \\
\text { Presión arterial diastólica } \leq 60\end{array}$ & 1 \\
\hline $\mathbf{m m H g}$ & Edad $\geq 65$ años & 1 \\
\hline
\end{tabular}

LMP: latidos por minuto.

\section{Interpretación}

\begin{tabular}{|c|c|l|}
\hline PUNTAJE & MORTALIDAD & \multicolumn{1}{|c|}{ RECOMENDACIÓN } \\
\hline $\mathbf{0}$ & $0.9 \%$ & $\begin{array}{l}\text { Bajo riesgo de muerte, atención } \\
\text { ambulatoria. }\end{array}$ \\
\hline $\mathbf{1}$ & $5.2 \%$ & $\begin{array}{l}\text { Riesgo intermedio de muerte, } \\
\text { considerar hospitalización. }\end{array}$ \\
\hline $\mathbf{2}$ & $12.0 \%$ & $\begin{array}{l}\text { Riesgo intermedio de muerte, } \\
\text { considerar hospitalización. }\end{array}$ \\
\hline $\mathbf{3 - 4}$ & $31.2 \%$ & $\begin{array}{l}\text { Alto riesgo de muerte, hospitalización } \\
\text { urgente. }\end{array}$ \\
\hline
\end{tabular}




\section{Bibliografía}

\section{Original/Primaria}

1. Alfageme I, Aspa J, Bello S, Blanquer J, Blanquer R, Borderías L et.al. Pautas para el diagnóstico y manejo de la neumonía adquirida en la comunidad. Sociedad Española de Neumología y Cirugía Torácica (SEPAR). Arch Bronconeumol [Internet]. 2005 mayo [citado marzo 2019];41(5):272-89. Disponible en: https: / / www.ncbi.nlm.nih.gov/ pubmed/15919009.

\section{Validación}

1. Vila A, Ochoa O, Rodriguez T. Utilidad de la escala CRB-65 en la evaluación pronóstica de los pacientes mayores de 65 años con neumonía adquirida en la comunidad. Med Clin [Internet]. 2010 [citado marzo 2019];135(3):97-102. Disponible en: https://www. elsevier.es / es-revista-medicina-clinica-2-articulo-utilidad-escala-crb65-evaluacion-pronostica-S0025775310000801. 


\section{PUNTAJE PSI O PORT}

\section{Utilidad}

Es PSI (Pneumonia Severity Index) o PORT (Patient Outcomes Research Team), una escala que permite estimar la mortalidad en pacientes adultos con neumonía adquirida en la comunidad.

\section{Descripción}

- Dado que se asignan puntos por la edad, se corre el riesgo de subestimar la neumonía grave en un paciente sano que, por lo demás, es joven.

- Si bien un paciente puede ser clasificado como apropiado para el tratamiento ambulatorio; evalúe las barreras potenciales al tratamiento, como vómitos, uso de alcohol / drogas, afecciones psicosociales o deficiencias cognitivas.

- Cualquier paciente mayor de 50 años de edad se clasifica automáticamente en la clase de riesgo 2, incluso si no tienen ningún otro criterio de riesgo.

\section{Parámetros}

\begin{tabular}{|l|c|}
\hline CRITERIO & PUNTOS \\
\hline Edad & Edad en años \\
Hombre & Edad -10 \\
Mujer & +10 \\
Internación domiciliaria & \\
\hline Co-morbilidad & 30 \\
Enfermedad neoplásica & 20 \\
Enfermedad hepática & 10 \\
Falla cardíaca congestiva & 10 \\
Enfermedad cerebro vascular & 10 \\
Enfermedad renal & \\
\hline
\end{tabular}




\begin{tabular}{|l|l|}
\hline Hallazgos al examen físico & \\
Alteración del estado mental & 20 \\
Frecuencia respiratoria $\geq 30 \mathrm{RPM}$ & 20 \\
Presión arterial sistólica $<90 \mathrm{mmHg}$ & 20 \\
Temperatura $<35^{\circ} \mathrm{C} \mathrm{o} \geq 40{ }^{\circ} \mathrm{C}$ & 15 \\
Frecuencia cardíaca $>125 \mathrm{LPM}$ & 10 \\
\hline Hallazgos analíticos y radiológicos & \\
PH arterial $<7,35$ & 30 \\
Urea $>64 \mathrm{mg} / \mathrm{dl}(\mathrm{BUN} \geq 30 \mathrm{mg} / \mathrm{dl})$ & 20 \\
Sodio $<130 \mathrm{mEq} / \mathrm{L}$ & 20 \\
Glucosa $\geq 250 \mathrm{mg} / \mathrm{dl}$ & 10 \\
Hematocrito $<30 \%$ & 10 \\
PO $<60 \mathrm{mmHg}$ o Sat $<90 \%$ & 10 \\
Derrame pleural & 10 \\
\hline
\end{tabular}

LPM: latidos por minuto, RPM: respiraciones por minuto.

\section{Interpretación}

\begin{tabular}{|l|l|l|l|l|}
\hline \multicolumn{1}{|c|}{ PUNTOS } & RIESGO & CLASE & $\begin{array}{l}\text { MORTALI- } \\
\text { DAD }\end{array}$ & TRATAMIENTO \\
\hline $\begin{array}{l}\text { Ni n q u a } \\
\text { comorbili- } \\
\text { dad }\end{array}$ & Bajo & I & $0.1 \%$ & Ambulatorio. \\
\hline $\mathbf{7 0}$ & Bajo & II & $0.6 \%$ & Ambulatorio. \\
\hline $\mathbf{7 1 - 9 0}$ & Bajo & III & $0.9 \%$ & $\begin{array}{l}\text { Observación por } \\
\text { dratación y trata- } \\
\text { miento parenteral. }\end{array}$ \\
\hline $\mathbf{9 1 - 1 3 0}$ & Moderado & IV & $9.3 \%$ & Internación. \\
\hline $\mathbf{> 1 3 0}$ & Alto & V & $27 \%$ & Internación (UCI). \\
\hline
\end{tabular}




\section{Bibliografía}

\section{Original/Primaria}

1. Bien MJ. Auble TE, Yealy DM. Hanusa BH, Weissfeld LA. Singer DE. et. Al. Una regla de predicción para identificar a los pacientes de bajo riesgo con neumonía adquirida en la comunidad. N Engl J Med [Internet]. 199723 de enero [citado marzo de 2019]; 336 (4): 243-50. Disponible en: https: / / www.ncbi.nlm.nih.gov / pubmed / 8995086?dopt=Abstract.

\section{Validación}

1. Shah BA, Ahmed W, Dhobi GN, Shah NN, Khusheed SQ, Haq I. Validez del índice de gravedad de neumonía y los sistemas de puntuación de severidad CURB-65 en neumonía adquirida en la comunidad en un entorno indígena. The Indian Journal of Chest Diseases \& Allied Sciences [Internet]. 2010 [citado marzo 2019]; 52.:10-17. Disponible en: http:/ / medind.nic.in/iae/t10/i1/iaet10i1p9.pdf. 


\section{ESACALA DRIP}

\section{Utilidad}

La escala DRIP (Drug Resistance in Pneumonia), predice el riesgo de neumonía adquirida en la comunidad debido a patógenos resistentes a los medicamentos (antibióticos).

\section{Descripción}

El objetivo de la puntuación DRIP, es determinar cuándo deben usarse los antibióticos de amplio espectro, tanto para ser eficaces con el tratamiento, como para evitar el aumento de la resistencia a los antibióticos.

\section{Parámetros}

$\left.\begin{array}{|l|c|}\hline \text { CRITERIOS } & \text { PUNTAJE } \\ \hline \text { Factores de riesgo mayores } & 2 \\ \hline \text { Uso de antibióticos dentro de los 60 días previos. } & 2 \\ \text { Residente de cuidados a largo plazo. } & 2 \\ \text { Alimentación por sonda. } & 2 \\ \text { Diagnóstico previo de neumonía farmacorresistente en } \\ \text { el último año. }\end{array}\right)$

PPI: inhibidor de la bomba de protones, SARM: staphylococcus aureus resistente a la meticilina. 


\section{Interpretación}

\begin{tabular}{|c|l|}
\hline PUNTOS & \multicolumn{1}{|c|}{ INTERPRETACIÓN } \\
\hline$<4$ & $\begin{array}{l}\text { Bajo riesgo de neumonía farmacorresistente. Considere el } \\
\text { tratamiento sin antibióticos de espectro extendido. }\end{array}$ \\
\hline$\geq 4$ & $\begin{array}{l}\text { Alto riesgo de neumonía farmacorresistente. Es probable } \\
\text { que se necesiten antibióticos de espectro extendido. }\end{array}$ \\
\hline
\end{tabular}

\section{Bibliografía}

\section{Original/Primaria}

1. Webb BJ, Dascomb K, Stenehjem E, et al. Derivación y validación multicéntrica de la resistencia a los medicamentos en la neumonía Puntuación de la predicción clínica. Agentes antimicrobianos quimioterapia [Internet]. 2016 [citado marzo 2019]; 60 (5): 2652-63. Disponible en: https://aac.asm.org/content/60/5/2652.

\section{Validación}

1. Webb BJ, Sorensen J, Mecham I, Buckel W, et al. Uso de antibióticos y resultados después de la implementación de la resistencia a los medicamentos en la puntuación de neumonía en pacientes con disfunción eréctil con neumonía de inicio en la comunidad. Chest [Internet]. [citado marzo 2019]; Disponible en: https://journal. chestnet.org/article/S0012-3692(19)31012-8/ references.

2. Shorr, AF, Zilberberg, MD, Reichley, R. et al., Validación de una puntuación clínica para evaluar el riesgo de patógenos resistentes en pacientes con neumonía que acuden al servicio de urgencias. Clin Infect Dis [Internet. 2012 [citado marzo 2019]; 54:193-198. Disponible en: https: / / www.ncbi.nlm.nih.gov/ pubmed / 22109951?dopt=Abstract. 


\section{ESCALA SMART-COP}

\section{Utilidad}

Es una escala, que evalúa la necesidad de apoyo respiratorio y/o vasopresores en la neumonía adquirida en la comunidad.

\section{Descripción}

Se debe usar en pacientes $\geq 18$ años con hallazgos clínicos y radiográficos compatibles con neumonía adquirida en la comunidad (NAC).

Puede ayudar a estratificar qué pacientes necesitan ingreso en la UCI. No estima la mortalidad.

\section{Parámetros}

\begin{tabular}{|c|l|c|}
\hline \multicolumn{2}{|c|}{ CRITERIO } & PUNTAJE \\
\hline $\begin{array}{c}\text { Low systolic } \\
\text { blood pressure }\end{array}$ & Presión arterial sistólica $<90 \mathrm{mmHg}$ & 2 \\
\hline $\mathbf{M}$ & $\begin{array}{c}\text { Compromiso multilobar (radiología } \\
\text { Multilobar chest } \\
\text { radiography } \\
\text { involvement }\end{array}$ & 1 \\
\hline $\begin{array}{c}\mathbf{A} * \\
\text { Low albumin } \\
\text { level }\end{array}$ & Albumina $<3,5 \mathrm{~g} /$ dl & 1 \\
\hline $\mathbf{R}$ & Frecuencia respiratoria \\
$\begin{array}{c}\text { High respiratory } \\
\text { rate }\end{array}$ & $\begin{array}{l}>50 \text { años, } \geq 25 \text { respiraciones } / \text { minuto } \\
\mathbf{T}\end{array}$ & 1 \\
\hline $\begin{array}{c}\text { Taños, } \geq 30 \text { respiraciones } / \text { minuto } \\
\mathbf{C}\end{array}$ & Taquicardia $\geq 125$ latidos/minuto & 1 \\
\hline \begin{tabular}{c} 
Confusion \\
\hline
\end{tabular} & Confusión & 1 \\
\hline
\end{tabular}




\begin{tabular}{|c|c|c|}
\hline $\begin{array}{l}\qquad \mathbf{O}^{*} \\
\text { Poor oxygenation }\end{array}$ & $\begin{array}{l}\text { Oxigenación } \\
\leq 50 \text { años } \mathrm{PaO}_{2}<70 \mathrm{mmHg} \text { o sat } \leq 93 \% \\
>50 \text { años } \mathrm{PaO}_{2}<60 \mathrm{mmHg} \text { o sat } \leq 90 \%\end{array}$ & 2 \\
\hline $\begin{array}{c}\mathbf{P}^{*} \\
\text { Low arterial } \mathbf{p H}\end{array}$ & $\mathrm{PH}$ arterial $<7,35$ & 2 \\
\hline
\end{tabular}

\section{Interpretación}

\begin{tabular}{|c|l|}
\hline PUNTOS & RIESGO/RECOMENDACIÓN \\
\hline $\mathbf{0 - 2}$ & $\begin{array}{l}\text { Bajo riesgo de requerimiento de asistencia } \\
\text { ventilatoria mecánica y/o vasopresores. }\end{array}$ \\
\hline $3-4$ & $\begin{array}{l}\text { Moderado riesgo de requerimiento de asistencia } \\
\text { ventilatoria mecánica y/o vasopresores. }\end{array}$ \\
\hline $5-6$ & $\begin{array}{l}\text { Alto riesgo de requerimiento de asistencia } \\
\text { ventilatoria mecánica y/o vasopresores. }\end{array}$ \\
\hline$\geq 7$ & $\begin{array}{l}\text { Muy alto riesgo de requerimiento de asistencia } \\
\text { ventilatoria mecánica y/o vasopresores. }\end{array}$ \\
\hline
\end{tabular}

${ }^{\star}$ En caso de no disponer de estos parámetros (albuminemia, $\mathrm{PaO}_{2}$ y pH) esta debe ser la interpretación:

\begin{tabular}{|c|l|}
\hline PUNTOS & RIESGO/RECOMENDACIÓN \\
\hline $\mathbf{0}$ & $\begin{array}{l}\text { Muy Bajo riesgo de requerimiento de asistencia } \\
\text { ventilatoria mecánica y/o vasopresores. }\end{array}$ \\
\hline $\mathbf{1}$ & $\begin{array}{l}\text { Bajo riesgo de requerimiento de asistencia } \\
\text { ventilatoria mecánica y/o vasopresores. }\end{array}$ \\
\hline $\mathbf{2}$ & $\begin{array}{l}\text { Moderado riesgo de requerimiento de asistencia } \\
\text { ventilatoria mecánica y/o vasopresores. }\end{array}$ \\
\hline $\mathbf{3}$ & $\begin{array}{l}\text { Alto riesgo de requerimiento de asistencia } \\
\text { ventilatoria mecánica y/o vasopresores. }\end{array}$ \\
\hline$\geq 4$ & $\begin{array}{l}\text { Muy alto riesgo de requerimiento de asistencia } \\
\text { ventilatoria mecánica y/o vasopresores. }\end{array}$ \\
\hline
\end{tabular}




\section{Bibliografía}

\section{Original/Primaria}

1. Charles PG, Wolfe R, Whitby M, Fine MJ, Fuller AJ, Stirling R et al. SMART-COP: una herramienta para predecir la necesidad de soporte respiratorio o vasopresor intensivo en la neumonía adquirida en la comunidad. Clin Infect Dis [Internet]. 2008 [citado marzo 2018];47(3):375-84. Disponible en: https://www.ncbi.nlm.nih.gov/ pubmed / 18558884 .

\section{Validación}

1. Martí C, Garin N, Grosgurin O, Poncet A, Combescure C, Carballo S, et al. Predicción de neumonía severa adquirida en la comunidad: una revisión sistemática y un metanálisis. Crit Care [Internet]. Julio 2012 [citado marzo 2019];16(4):141. Disponible en: https: / / www.ncbi.nlm. nih.gov/pubmed/22839689.

2. Valley TS, Sjoding MW, Ryan AM, Iwashyna TJ, Cooke CR. Asociación de ingreso a la unidad de cuidados intensivos con mortalidad en pacientes ancianos con neumonía. JAMA [Internet] 2015 Septiembre [citado marzo 2019]; 22-29; 314(12):1272-9. Disponible en: https:// www.ncbi.nlm.nih.gov/pubmed/26393850.

3. Restrepo MI, Mortensen EM, Rello J, Brody J, Anzueto A. La admisión tardía a la UCI en pacientes con neumonía adquirida en la comunidad se asocia con una mayor mortalidad. Chest [Internet]. Marzo 2010 [citado marzo 2019]:137(3):552-557. Disponible en: https://www. ncbi.nlm.nih.gov/pmc/articles/PMC3021366/. 


\section{CRITERIOS DE ALIBERTI PARA NEUMONÍA EN EL ADULTO MAYOR}

\section{Utilidad}

Es una herramienta que permite evaluar, en adultos mayores, la probabilidad de tener un patógeno multidrogoresistente.

\section{Parámetros}

\begin{tabular}{|l|c|}
\hline CRITERIOS & PUNTAJE \\
\hline $\begin{array}{l}\text { No hay factores de riesgo para el patógeno MDR (incluidas } \\
\text { las comorbilidades) }\end{array}$ & 0 \\
\hline $\begin{array}{l}\text { > de las siguientes comorbilidades: enfermedad } \\
\text { cerebrovascular, diabetes, EPOC, terapia antimicrobiana } \\
\text { en los anteriores 90 días, inmunosupresión, cuidado de } \\
\text { heridas a domicilio, terapia de intravenosa en el hogar } \\
\text { (incluyendo antibióticos). }\end{array}$ & 0.5 \\
\hline $\begin{array}{l}\text { Residencia en un hogar de ancianos o centro de cuidados } \\
\text { prolongados. }\end{array}$ & 3 \\
\hline Hospitalización por $>2$ días en los últimos 90 días . & 4 \\
\hline Falla renal crónica. & 5 \\
\hline
\end{tabular}

\section{Interpretación}

\begin{tabular}{|c|c|}
\hline PUNTOS & Probabilidad de MDR \\
\hline $\mathbf{0 - 0 . 5}$ & Bajo riesgo \\
\hline $3-12.5$ & Alto riesgo \\
\hline
\end{tabular}




\section{Bibliografía}

\section{Original/Primaria}

1. AlibertiS,DiPasqualeM,ZanaboniAN,Cosentini R, etal.Estratificación de los factores de riesgo para patógenos multirresistentes en pacientes hospitalizados procedentes de la comunidad con neumonía.. Clinical Infectious diseases [Internet]. 2012 [citado marzo 2019]:54(4);470-8. Disponible en: https: / / www.ncbi.nlm.nih.gov/pubmed/ 22109954

\section{Validación}

1. Aliberti S, Cilloniz C, Chalmers JD, Zanaboni AM, Cosentini R, et al. Patógenos multirresistentes en pacientes hospitalizados procedentes de la comunidad con neumonía: una perspectiva europea. Thorax [Internet]. 2013 [citado marzo 2019] ;68(11):997-9. Disponible en: https: / / www.ncbi.nlm.nih.gov/ pubmed/23774884. 


\section{NEUMONÍA NOSOCOMIAL}

\section{SCORE SHORR PARA RIESGO DE SARM EN PACIENTES CON NEUMONÍA}

\section{Utilidad}

Identifica a pacientes con riesgo de neumonía por SARM (Staphylococcus aureus resistente a la meticilina).

\section{Descripción}

El Shorr Score se desarrolló para evaluar el riesgo de neumonía por SARM y determinar quién podría beneficiarse al agregar la cobertura para estos patógenos.

\section{Parámetros}

\begin{tabular}{|l|c|}
\hline CRITERIO & PUNTAJE \\
\hline Edad 19-29 o $>79$ años & 1 \\
\hline $\begin{array}{l}\text { Hogar de ancianos, centro de enfermería especializada o } \\
\text { exposición a cuidados intensivos a largo plazo (dentro de } \\
\text { 90 días). }\end{array}$ & 1 \\
\hline Terapia antibiótica IV previo en los últimos 30 días. & 1 \\
\hline Hospitalización por $\geq 2$ días, en los últimos 90 días. & 2 \\
\hline Admisión en la UCI. & 2 \\
\hline $\begin{array}{l}\text { Cualquier enfermedad cerebrovascular antes de la } \\
\text { admisión. }\end{array}$ & 1 \\
\hline Demencia. & 1 \\
\hline Mujer con Diabetes Mellitus. & 1 \\
\hline
\end{tabular}

\section{Interpretación}

\section{PUNTOS RIESGO/RECOMENDACIÓN}

\begin{tabular}{|c|l|}
\hline $\mathbf{0 - 1}$ & $\begin{array}{l}\text { Bajoriesgo deSARM. Considerar la cobertura de antibióticos } \\
\text { de rutina. }\end{array}$ \\
\hline $\mathbf{2 - 5}$ & $\begin{array}{l}\text { Riesgo intermedio de SARM. Utilizar el juicio clínico con } \\
\text { respecto a la cobertura de antibióticos. }\end{array}$ \\
\hline
\end{tabular}




\section{Bibliografía}

\section{Original/Primaria}

1. Shorr AF, Myers DE, Huang DB, Nathanson BH, Emons MF, Kollef MH. Una puntuación de riesgo para la identificación de Staphylococcus aureus resistente a la meticilina en pacientes que acuden al hospital con neumonía. BMC Infect Dis [Internet]. 2013 [citado marzo 2019]; 13: 268. Disponible en: https://bmcinfectdis.biomedcentral.com/ articles / 10.1186/1471-2334-13-268.

\section{Validación}

1. Minejima E, Lou M, Nieberg P, Wong-beringer A. Pacientes que se presentan en el hospital con neumonía por SARM: características diferenciales y resultados con tratamiento empírico. BMC Infect Dis [Internet]. 2014 [citado marzo 2019];14: 252. Disponible en: https:/ / www.ncbi.nlm.nih.gov/pubmed/24885158.

2. Teshome BF, Lee GC, Reveles KR, Attridge RT, Koeller J, Wang CP, et al. Aplicación de una puntuación de riesgo de Staphylococcus aureus resistente a la meticilina en pacientes con neumonía de inicio en la comunidad y resultados con tratamiento inicial. BMC Infect Dis [Internet]. 2015 [citado marzo 2019]:15: 380. Disponible en: https:// www.ncbi.nlm.nih.gov/pubmed/26385225.

3. Shorr AF, Zilberberg MD, Reichley R, Kan J, Hoban A, Hoffman $\mathrm{J}$, et al. Validación de una puntuación clínica para evaluar el riesgo de patógenos resistentes en pacientes con neumonía que acuden al servicio de urgencias. Clin Infect Dis [Internet]. 2012 [citado marzo 2019];54(2):193-8. Disponible en: https://www.ncbi.nlm.nih.gov/ pubmed / 22109951. 


\section{Utilidad}

Ayuda a diagnosticar la neumonía asociada a ventilación mecánica y predice el beneficio de los cultivos pulmonares.

\section{Parámetros}

\begin{tabular}{|c|c|}
\hline CRITERIOS & PUNTAJE \\
\hline \multicolumn{2}{|l|}{ Temperatura } \\
\hline $36.5-38.4$ & 0 \\
\hline $38.5-38.9$ & 1 \\
\hline$\geq 39.0 \mathrm{o} \leq 36.0$ & 2 \\
\hline \multicolumn{2}{|l|}{ Recuento de glóbulos blancos } \\
\hline $4000-11000$ & 0 \\
\hline$<4000$ o $>11000$ & 1 \\
\hline Bandas igual o $>50 \%$ & 2 \\
\hline \multicolumn{2}{|l|}{ Secreciones traqueales } \\
\hline Ausencia de secreciones & 0 \\
\hline Presencia de secreciones no purulentas & 1 \\
\hline Presencia de secreciones purulentas & 2 \\
\hline \multicolumn{2}{|l|}{ Oxigenación $\mathrm{PaO}_{2} / \mathrm{FiO}_{2} \mathrm{~mm} \mathrm{Hg}$} \\
\hline $\mathrm{PaO}_{2} / \mathrm{FiO}_{2}>240$ o SDRA & 0 \\
\hline $\mathrm{PaO}_{2} / \mathrm{FiO}_{2} \leq 240$ y $\sin$ SDRA & 2 \\
\hline
\end{tabular}




\begin{tabular}{|l|l|}
\hline Radiografía pulmonar & \\
No infiltrado & 0 \\
Infiltrado difuso & 1 \\
Infiltrado localizado & 2 \\
\hline Cultivo de muestra de aspirado traqueal & \\
Poco o nulo crecimiento & 0 \\
Crecimiento moderado o intenso & 1 \\
Visualización en el Gram & 2 \\
\hline
\end{tabular}

PaO2/FiO2: Presión Arterial de Oxígeno/fracción inspirada de oxígeno.

SDRA: Síndrome del Distres Respiratorio del Adulto.

\section{Interpretación}

\section{PUNTOS}

\begin{tabular}{|c|l|}
\hline $\mathbf{0}$ - $\mathbf{6}$ & $\begin{array}{l}\text { Bajo riesgo de neumonía asociada a ventilación } \\
\text { mecánica }\end{array}$ \\
\hline$\geq 7$ & $\begin{array}{l}\text { Alto riesgo de neumonía asociada a ventilación } \\
\text { mecánica }\end{array}$ \\
\hline
\end{tabular}

\section{Bibliografía}

\section{Original/Primaria}

1. Schurink CA, Van Nieuwenhoven CA, Jacobs JA, Rozenberg-Arska M, Joore HC, Buskens E, Hoepelman AI, Bonten MJ. Puntuación clínica de infección pulmonar para neumonía asociada a ventilación mecánica: precisión y variabilidad interobservador. Cuidados intensivos med [Internet]. Febrero de 2004 [citado marzo 2019]; 30 (2): 217-24. Disponible en: https: / / www.ncbi.nlm.nih.gov/pubmed/14566455.

\section{Validación}

1. Fartoukh M, Maitre B, HonoréS, Cerf C, Zahar JR, Brun-Buisson C. Diagnóstico de la neumonía durante la ventilación mecánica: revisión 
de la puntuación de la infección clínica pulmonar. Am J Respir Crit Care Med [Internet]. 2003 [citado marzo 2019];168(2):173. Disponible en: https: / / www.ncbi.nlm.nih.gov/pubmed/12738607.

2. Shan J, Chen HL, Zhu JH. Precisión diagnóstica de la puntuación clínica de infección pulmonar para la neumonía asociada a ventilador: un metanálisis.Respir Care [Internet]. 2011 [citado marzo 2019]. ;56(8):1087-94. Disponible en: https: / / www.ncbi.nlm.nih.gov/ pubmed/21310117.

3. Parques NA, Magnotti LJ, Weinberg JA, Zarzaur BL, Schroeppel TJ, et al. El uso de la puntuación clínica de infección pulmonar para guiar la terapia para la neumonía asociada a la ventilación conlleva un riesgo de sobreexposición a los antibióticos en pacientes con traumatismo. J Trauma Acute Care Surg [Internet]. 2012 [citado marzo 2019]. Disponible en: https: / / www.ncbi.nlm.nih.gov/pubmed/ 22743372. 


\section{INFLUENZA AH1N1}

SISTEMA DE PUNTAJE DEL HOSPITAL UNIVERSITARIO WINTHROP-DIVISIÓN DE ENFERMEDADES INFECCIOSAS, PARA EL DIAGNÓSTICO DE INFLUENZA SEVERA EN ADULTOS (MODIFICADO)

\section{Utilidad}

El sistema Winthrop es una herramienta diagnóstica fundamentada en signos, síntomas y pruebas de laboratorio e imagen para identificar pacientes con moderada y alta sospecha de neumonía por influenza AH1N1.

\section{Parámetros}

\section{CRITERIOS}

\begin{tabular}{|l|c|}
\hline Síntomas & PUNTAJE \\
\hline Inicio hiperagudo & +3 \\
\hline Severa postración & +5 \\
\hline Dolores musculares generalizados & +3 \\
\hline Dolor retroorbitario & +5 \\
\hline Dolores severos de cuello, espalda y región lumbar & +5 \\
\hline Signos & \\
\hline Fiebre mayor a 38.5 C & +2 \\
\hline Tos seca & +1 \\
\hline Congestión conjuntival & +5 \\
\hline Hemoptisis & +3 \\
\hline Estertores localizados & -3 \\
\hline Cianosis & +5 \\
\hline Test de laboratorio & \\
\hline Leucocitosis & -5 \\
\hline
\end{tabular}




\begin{tabular}{|l|c|}
\hline Lucopenia & +3 \\
\hline Linfopenia relativa & +3 \\
\hline Trombocitopenia & +3 \\
\hline $\begin{array}{l}\text { Rx estándar de tórax sin infiltración o mínimos infiltrados, } \\
\text { dentro de las primeras 8 horas. }\end{array}$ & +3 \\
$\begin{array}{l}\text { Infiltrados irregulares bilaterales, en un tiempo mayor 48 } \\
\text { horas. }\end{array}$ & +3 \\
$\begin{array}{l}\text { Infiltrados focales o segmentarios (consolidaciones } \\
\text { locales). }\end{array}$ & -5 \\
\hline
\end{tabular}

\section{Interpretación}

\begin{tabular}{|c|l|}
\hline PUNTOS & RIESGO \\
\hline $\mathbf{2 0}$ & Alta probabilidad de Influenza A \\
\hline $\mathbf{1 0 - 2 0}$ & Leve a moderada probabilidad de influenza A \\
\hline$<\mathbf{1 0}$ & Poca probabilidad de Influenza A \\
\hline
\end{tabular}

\section{Bibliografía}

\section{Original/Primaria}

1. Cunha BA. El diagnóstico clínico de influenza viral severa A. Infection [Internet]. 2008 [citado marzo 2019] ; 36 (1): 92-3. Disponible en: https: / / www.ncbi.nlm.nih.gov/pubmed/18231716.

\section{Validación}

1. Cunha BA, Syed U, Paseo S, Mickail N, Laguerre M. WinthropUniversity Hospital Infectious Disease Division's swine influenza (H1N1) pneumonia diagnostic weighted point score system for hospitalized adults with influenza-like illnesses (ILIs) and negative rapid influenza diagnostic tests (RIDTs). Heart Lung [Internent]. 2009 [citado marzo 2019];38(6):534-8. Disponible en: https://www.ncbi. nlm.nih.gov/pubmed/19944879. 


\section{EPOC EXACERBADO}

\section{ESCALA DE DISNEA (MMRC) MODIFICADA}

\section{Utilidad}

Estratifica la severidad de la disnea en las enfermedades respiratorias, particularmente la enfermedad pulmonar obstructiva crónica (EPOC).

\section{Descripción}

- La escala mMRC cuantifica la discapacidad atribuible a la disnea y es útil para caracterizar la disnea basal en pacientes con enfermedades respiratorias.

- Describe la disnea inicial, pero no cuantifica con precisión la respuesta al tratamiento de la enfermedad pulmonar obstructiva crónica (EPOC).

- Se recomienda evaluar el caminar del paciente en terreno llano.

\section{Parámetros}

\begin{tabular}{|l|c|}
\hline SEVERIDAD DE LOS SÍNTOMAS & PUNTAJE \\
\hline Ausencia de disnea excepto al realizar ejercicio intenso. & 0 \\
\hline Disnea cuando se apresura o sube una cuesta. & 1 \\
\hline $\begin{array}{l}\text { Camina más lento que las personas de la misma edad } \\
\text { debido a la disnea o tiene que detenerse para respirar } \\
\text { cuando camina a su propio ritmo. }\end{array}$ & 2 \\
\hline $\begin{array}{l}\text { Tiene que parar a descansar al andar unos 100 metros o a } \\
\text { los pocos minutos de andar en llano. }\end{array}$ & 3 \\
\hline $\begin{array}{l}\text { La disnea impide al paciente salir de casa, o aparece con } \\
\text { actividades como bañarse o vestirse. }\end{array}$ & 4 \\
\hline
\end{tabular}




\section{Interpretación}

\section{PUNTOS}

GRADO

\begin{tabular}{|c|c|}
\hline \multicolumn{2}{|c|}{ Mayor grado indica mayor severidad } \\
\hline $\mathbf{1}$ & $\mathrm{I}$ \\
\hline 2 & $\mathrm{II}$ \\
\hline 3 & $\mathrm{III}$ \\
\hline 4 & $\mathrm{IV}$ \\
\hline
\end{tabular}

\section{Bibliografía}

\section{Original/Primaria}

1. Mahler DA, Wells CK. Evaluación de métodos clínicos para la calificación de la disnea. Chest [Internet. 1988 marzo [citado marzo 2019]; 93 (3): 580-6. Disponible en: https: / / www.ncbi.nlm.nih.gov / pubmed/3342669.

\section{Validación}

1. Hajiro T, Nishimura K, Tsukino M, Ikeda A, Koyama H, Izumi T. Análisis de los métodos clínicos utilizados para evaluar la disnea en pacientes con enfermedad pulmonar obstructiva crónica. Am J Respir Crit Care Med [Internet]. 1998 [citado marzo 2019];158(4):1185-9. Disponible en: https:/ / www.ncbi.nlm.nih.gov/pubmed/9769280.

2. Nishiyama O, Taniguchi H, Kondoh Y, Kimura T, Kato K, Kataoka $\mathrm{K}$,et al. Una evaluación simple de la disnea como indicador pronóstico en la fibrosis pulmonar idiopática. Eur Respir J [Internet]. 2010 [citado marzo 2019]. 36 (5): 1067-72. Disponible en: https:/ / www.ncbi.nlm. nih.gov/pubmed/20413545

3. Launois C, Barbe C, Bertin E, Nardi J, Perotin JM, Dury S, et al. La escala modificada del Consejo de Investigación Médica para la evaluación de la disnea en la vida diaria en la obesidad: un estudio piloto. BMC Medicina Pulmonar [Internet]. 2012 [citado marzo 2019]. Disponible en: https://bmcpulmmed.biomedcentral.com/ articles / 10.1186/1471-2466-12-61. 
4. Celli BR, Cote CG, Marin JM, Casanova C. El índice de masa corporal, la obstrucción del flujo de aire, la disnea y el índice de capacidad de ejercicio en la enfermedad pulmonar obstructiva crónica. N Engl J Med [Internet]. 2004 [citado marzo 2019]. Disponible en: https:// www.nejm.org/ doi / full/10.1056/NEJMoa021322. 


\section{Utilidad}

Esta escala predice la mortalidad en la exacerbación aguda de la EPOC.

\section{Descripción}

- Se debe usar en pacientes $>40$ años de edad que acuden al servicio de urgencias con exacerbación aguda de la EPOC.

- Solo se debe usar en pacientes que se presentan en el servicio de urgencias con un diagnóstico primario de exacerbación aguda de la EPOC, no en el contexto ambulatorio o en pacientes cuya EPOC es estable.

- Puede ayudar en la toma de decisiones clínicas para estratificar a los pacientes a un nivel más alto de atención, o potencialmente observación o alta temprana.

\section{Parámetros}

\begin{tabular}{|l|c|}
\hline \multicolumn{2}{|c|}{ CRITERIO } \\
\hline \multicolumn{2}{|c|}{$\begin{array}{l}\text { Edad } \\
265\end{array}$} \\
\hline & PUNTAJE \\
\hline BUN $\geq 25 \mathrm{mg} / \mathrm{dL}(8.9$ mmol / L). & 1 \\
\hline $\begin{array}{l}\text { Estado mental alterado (GCS inicial }<14, \text { o desorientación, } \\
\text { estupor o coma según lo determine el médico). }\end{array}$ & 1 \\
\hline Pulso $\geq 109$ latidos / min. & 1 \\
\hline
\end{tabular}

GSC: Escala de coma de Glasgow. 


\section{Interpretación}

\begin{tabular}{|c|c|c|c|c|c|}
\hline Puntos & Grado & Años & $\begin{array}{l}\text { Mortalidad } \\
\text { hospitalaria }\end{array}$ & 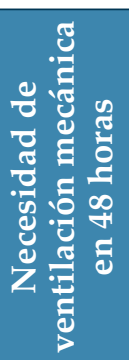 & Recomendación \\
\hline 0 & I & $<65$ años & $0.3 \%$ & $0.3 \%$ & \multirow{2}{*}{$\begin{array}{l}\text { Manejo rutinario de la } \\
\text { exacerbación de la EPOC. }\end{array}$} \\
\hline 0 & II & $\geq 65$ años & $1 \%$ & $0.2 \%$ & \\
\hline 1 & III & $\begin{array}{l}\text { Cualquier } \\
\text { edad }\end{array}$ & $2.2 \%$ & $1.2 \%$ & \multirow{3}{*}{$\begin{array}{l}\text { Considere la posibilidad } \\
\text { de ventilación temprana } \\
\text { no invasiva y/o cuidados } \\
\text { de UCI. }\end{array}$} \\
\hline 2 & IV & $\begin{array}{l}\text { Cualquier } \\
\text { edad }\end{array}$ & $6.4 \%$ & $5.5 \%$ & \\
\hline 3 & V & $\begin{array}{l}\text { Cualquier } \\
\text { edad }\end{array}$ & $14.1 \%$ & $12.4 \%$ & \\
\hline
\end{tabular}

EPOC: enfermedad pulmonar obstructiva crónica; UCI: unidad de cuidados intensivos.

\section{Bibliografía}

\section{Original/Primaria}

1. Tabak YP, Sun X, Johannes RS, Gupta V, Shorr AF. Mortalidad y necesidad de ventilación mecánica en las exacerbaciones agudas de la enfermedad pulmonar obstructiva crónica: desarrollo y validación de una puntuación de riesgo simple. Arch Intern Med [Internet]. 2009 [citado marzo 2019]; 169 (17): 1595-602. Disponible en: https: / /jamanetwork.com/journals/jamainternalmedicine/ fullarticle/ 224759.

\section{Validación}

1. Shorr AF, Sun X, Johannes RS, Yaitanes A, Tabak YP. Validación de una nueva puntuación de riesgo para la gravedad de la enfermedad en las exacerbaciones agudas de la EPOC. Chest [Internet]. 2011 [citado marzo 2019]; 140 (5): 1177-1183. Disponible en: https://www.ncbi. nlm.nih.gov/pubmed/21527510. 
2. Shorr AF, Sun X, Johannes RS, Derby KG, Tabak YP. Predicción de la necesidad de ventilación mecánica en las exacerbaciones agudas de la enfermedad pulmonar obstructiva crónica: comparación de las puntuaciones CURB-65 y BAP-65. J Crit Care [Internet]. 2012 [citado marzo 2019]. Disponible en: https: / / www.ncbi.nlm.nih.gov/ pubmed / 22520489. 


\section{ÍNDICE BODE}

\section{Utilidad}

El índice BODE predice la supervivencia en pacientes con EPOC.

\section{Descripción}

- No usar en pacientes durante las exacerbaciones agudas de la EPOC.

- No utilizar para guiar la terapia.

- Diseñado para su uso en pacientes con EPOC estable que ya reciben el tratamiento adecuado.

\section{Parámetros}

\begin{tabular}{|l|c|}
\hline CRITERIO & PUNTAJE \\
\hline FEV $_{\mathbf{1}}$ & 0 \\
\hline$\geq 65 \%$ & 1 \\
$50-64 \%$ & 2 \\
$36-49 \%$ & 3 \\
$\leq 35 \%$ & \\
\hline Distancia de caminata de 6 minutos & 0 \\
\hline$\geq 350 \mathrm{~m}$ & 1 \\
$250-349 \mathrm{~m}$ & 2 \\
$150-249 \mathrm{~m}$ & 3 \\
$\leq 149 \mathrm{~m}$ & \\
\hline
\end{tabular}




\begin{tabular}{|c|c|}
\hline Escala de disnea mMRC & \\
\hline Ausencia de disnea excepto al realizar ejercicio intenso. & 0 \\
\hline Disnea cuando se apresura o sube una cuesta. & +1 \\
\hline $\begin{array}{l}\text { Camina más lento que las personas de la misma edad } \\
\text { debido a la disnea o tiene que detenerse para respirar } \\
\text { cuando camina a su propio ritmo. }\end{array}$ & +2 \\
\hline $\begin{array}{l}\text { Tiene que parar a descansar al andar unos } 100 \text { metros o a } \\
\text { los pocos minutos de andar en llano. }\end{array}$ & +3 \\
\hline $\begin{array}{l}\text { La disnea impide al paciente salir de casa, o aparece con } \\
\text { actividades como bañarse o vestirse. }\end{array}$ & +4 \\
\hline $\operatorname{IMC}\left(\mathrm{kg} / \mathrm{m}^{2}\right)$ & \\
\hline$>21$ & 0 \\
\hline$\leq 21$ & 1 \\
\hline
\end{tabular}

FEV: volumen espiratorio forzado, IMC: indice de masa corporal.

\section{Interpretación}

\begin{tabular}{|c|c|}
\hline PUNTOS & SUPERVIVENCIA A 4 AñOS \\
\hline $\mathbf{0 - 2}$ & $80 \%$ \\
\hline $\mathbf{3 - 4}$ & $67 \%$ \\
\hline $\mathbf{5 - 6}$ & $57 \%$ \\
\hline $\mathbf{7 - 1 0}$ & $18 \%$ \\
\hline
\end{tabular}

\section{Bibliografía}

\section{Original/Primaria}

1. Celli BR. El índice de masa corporal, la obstrucción del flujo de aire, la disnea y el índice de capacidad de ejercicio en la enfermedad pulmonar obstructiva crónica. N Engl J M [Internet]. 2004 [citado marzo 2019]; 350: 10; 1005-1012. Disponible en: https:/ / www.nejm. org/doi / full/10.1056/ NEJMoa021322\#t=article. 


\section{Validación}

1. Marin JM, Carrizo SJ, Casanova C, et al. Predicción del riesgo de exacerbaciones de la EPOC por el índice BODE. Respir Med [Internet]. 2009 [citado marzo 2019];103(3):373-8. Disponible en: https:/ / www. ncbi.nlm.nih.gov/pubmed/19013781.

2. Mahler DA, Wells CK. Evaluación de métodos clínicos para la calificación de la disnea. Chest [Internet]. 1988 [citado marzo 2019]. Disponible en: https: / / www.ncbi.nlm.nih.gov/pubmed/3342669. 


\section{Utilidad}

La escala CAT (Copd Assessment Test), cuantifica el impacto de los síntomas de la EPOC en la salud general de los pacientes.

\section{Descripción}

Usar en pacientes diagnosticados con EPOC, para evaluar la progresión de la enfermedad pulmonar, la disminución del estado funcional y la eficacia de la rehabilitación pulmonar.

\section{Parámetros}

\begin{tabular}{|c|c|c|c|}
\hline SÍNTOMA & PTS & SÍNTOMA & PTS \\
\hline $\begin{array}{l}\text { Tos } \\
\text { Nunca toso. }\end{array}$ & $\begin{array}{l}0 \\
1 \\
2 \\
3 \\
4 \\
5\end{array}$ & $\begin{array}{l}\text { Ocupaciones } \\
\text { No estoy limitado en hacer } \\
\text { ninguna actividad en casa. } \\
\text { Estoy muy limitado haciendo } \\
\text { actividades en casa. }\end{array}$ & $\begin{array}{l}0 \\
1 \\
2 \\
3 \\
4 \\
5\end{array}$ \\
\hline $\begin{array}{l}\text { Flema } \\
\text { No tengo flema en } \mathrm{mi} \\
\text { pecho. }\end{array}$ & $\begin{array}{l}0 \\
1 \\
2 \\
3 \\
4 \\
5\end{array}$ & $\begin{array}{l}\text { Confianza } \\
\text { Tengo confianza en dejar } \\
\text { mi hogar a pesar de mi } \\
\text { enfermedad pulmonar. }\end{array}$ & $\begin{array}{l}0 \\
1 \\
2 \\
3 \\
4 \\
5\end{array}$ \\
\hline
\end{tabular}




\begin{tabular}{|l|l|l|l|}
\hline Opresión en el pecho & & Energía & \\
$\begin{array}{l}\text { Mi pecho no se siente } \\
\text { apretado en absoluto. }\end{array}$ & 0 & Tengo mucha energía. & 0 \\
& 2 & & 1 \\
& 3 & & 2 \\
$\begin{array}{l}\text { Mi pecho se siente muy } \\
\text { apretado. }\end{array}$ & 5 & No tengo energía. & 3 \\
\hline $\begin{array}{l}\text { Disnea una } \\
\text { Cuando subo undo } \\
\text { colina o un tramo de } \\
\text { escaleras no me quedo } \\
\text { sin aliento. }\end{array}$ & 0 & Duermo profundamente. & 4 \\
& 1 & & 5 \\
\hline $\begin{array}{l}\text { Cuando subo una } \\
\text { colina o un tramo de } \\
\text { escaleras me quedo sin } \\
\text { aliento. }\end{array}$ & 4 & No puedo dormir debido a mi & 4 \\
\hline
\end{tabular}

\section{Interpretación}

\begin{tabular}{|l|l|l|}
\hline PUNTOS & $\begin{array}{l}\text { IMPACTO } \\
\text { EN LA } \\
\text { SALUD }\end{array}$ & \multicolumn{1}{|c|}{ RECOMENDACIÓN } \\
\hline $\mathbf{0 - 1 0}$ & Bajo & $\begin{array}{l}\text { Dejar de fumar, atención preventiva y } \\
\text { menor exposición a factores de riesgo } \\
\text { de exacerbación; considerar LAMA y los } \\
\text { inhaladores de rescate. }\end{array}$ \\
\hline $\mathbf{1 1 - 2 0}$ & Medio & $\begin{array}{l}\text { Dejar de fumar, atención preventiva, } \\
\text { menor exposición a factores de riesgo } \\
\text { de exacerbación, e inhaladores LAMA } \\
\text { y de rescate; considerar ICS y/o LABA, } \\
\text { referencias para rehabilitación pulmonar y } \\
\text { posible evaluación de trasplante de pulmón. }\end{array}$ \\
\hline
\end{tabular}




\begin{tabular}{|l|l|l|}
\hline 21-30 & Alto & $\begin{array}{l}\text { Dejar de fumar, atención preventiva, } \\
\text { reducción de la exposición a factores de riesgo } \\
\text { de exacerbación, terapia con ICS / LABA } \\
\text { / LAMA, derivaciones para rehabilitación } \\
\text { pulmonar, posible evaluación de trasplante } \\
\text { de pulmón y suplementos de } \mathrm{O}_{2} .\end{array}$ \\
\hline
\end{tabular}

LAMA: antimuscarínicos de acción prolongada; LABA: beta-2 agonistas de larga duración; ICS: corticoesteroides inhalados.

\section{Bibliografía}

\section{Original/Primaria}

1. Jones PW, Harding G, Berry P, Wiklund I, Chen WH, Kline Leidy N. Desarrollo y primera validación de la prueba de evaluación de la EPOC. Eur Respir J [Internet]. 2009 [citado marzo 2019]; 34 (3): 648-54. Disponible en: https: / / www.ncbi.nlm.nih.gov/pubmed/19720809.

\section{Validación}

1. Dodd JW, Hogg L, Nolan J, et al. Prueba de evaluación de la EPOC (CAT): respuesta a la rehabilitación pulmonar. Un estudio multicéntrico, prospectivo. Thorax [Internet]. 2011 [citado 27 marzo 2019]; 66 (5): 425-9. Disponible en: https:/ / www.ncbi.nlm.nih.gov/ pubmed / 21398686 .

2. Husebo G, Koll RM, Fleten A, Maestad K, Krekvik A. La puntuación CAT es un predictor de mortalidad en la EPOC. Eur Respir J [Internet]. 2016 [citado marzo 2019]. 48: PA3106. Disponible en: https://erj. ersjournals.com/content/48/suppl_60/PA3106. 


\section{Utilidad}

Esta escala predice la mortalidad intrahospitalaria en la exacerbación aguda de la EPOC.

\section{Descripción}

- Uso en pacientes $\geq 35$ años, hospitalizados con diagnóstico primario de exacerbación aguda de la EPOC.

- No usar en pacientes con comorbilidad que se espera que limiten la supervivencia $<12$ meses.

- Mejor predictor de mortalidad intrahospitalaria que el CURB-65 en pacientes con neumonía y exacerbación aguda de la EPOC.

\section{Parámetros}

\begin{tabular}{|l|c|}
\hline CRITERIO & PUNTAJE \\
\hline Escala de disnea MRC extendia (eMRCD) & 0 \\
No es demasiada disnea para salir de casa. & 1 \\
$\begin{array}{l}\text { Demasiada disnea para salir de casa, pero independiente } \\
\text { de bañarse o vestirse. }\end{array}$ & 2 \\
\hline Demasiada disnea para salir de casa, bañarse o vestirse. & 1 \\
\hline Eosinopenia & 1 \\
\hline Eosinófilos $<0.05 \times 10^{9} /$ L. & 1 \\
\hline Consolidación en radiografía de tórax & 1 \\
\hline Acidemia & \\
\hline pH $<7.30$ & Fibrilación auricular \\
En EKG en la presentación y/o historia de fa paroxística. & \\
\hline
\end{tabular}




\section{Interpretación}

\begin{tabular}{|c|c|c|c|}
\hline PUNTOS & RIESGO & RECOMENDACIÓN & $\begin{array}{l}\text { MORTALIDAD } \\
\text { HOSPITALARIA }\end{array}$ \\
\hline $\mathbf{0}$ & \multirow{2}{*}{ Bajo } & \multirow{2}{*}{ Gestión de rutina } & $0 \%$ \\
\hline 1 & & & $15 \%$ \\
\hline 2 & Intermedio & $\begin{array}{l}\text { Utilizar el criterio } \\
\text { clínico en relación con la } \\
\text { disposición }\end{array}$ & $5.4 \%$ \\
\hline 3 & \multirow{4}{*}{ Alto } & \multirow{4}{*}{$\begin{array}{l}\text { Considere la escalada } \\
\text { de atención frente a los } \\
\text { cuidados paliativos }\end{array}$} & $15.3 \%$ \\
\hline 4 & & & $31 \%$ \\
\hline 5 & & & $40.5 \%$ \\
\hline 6 & & & $50 \%$ \\
\hline
\end{tabular}

\section{Bibliografía}

\section{Original/Primaria}

1. Dirección J, Gibson J, Bourke SC. Puntuación DECAF: predicción de la mortalidad hospitalaria en exacerbaciones de la enfermedad pulmonar obstructiva crónica. Thorax [Internet]. 2012 [citado marzo 2019]; 67 (11): 970-6. Disponible en: https: / / www.ncbi.nlm.nih.gov/ pubmed/22895999.

\section{Validación}

1. Echevarria C, Steer J, Heslop-marshall K, et al. Validación de la puntuación DECAF para predecir la mortalidad hospitalaria en exacerbaciones agudas de la EPOC. Thorax [Internet]. 2016 [citado marzo 2019]; 71 (2): 133-40. Disponible en: https://www.ncbi.nlm. nih.gov/pubmed/26769015. 


\section{CRITERIOS GOLD (GLOBAL INITIATIVE FOR OBSTRUCTIVE LUNG DISEASE) PARA EPOC}

\section{Utilidad}

Evalúa las diferentes etapas de la EPOC y proporciona recomendaciones de tratamiento.

\section{Descripción}

- Usar en pacientes mayores 18 años de edad, con EPOC ya diagnosticada por espirometría (FEV 1 / FVC $<0.7)$, con síntomas basales y función pulmonar.

- No utilice para diagnosticar la EPOC y no utilice en pacientes con exacerbación aguda.

\section{Parámetros}

\begin{tabular}{|l|l|}
\hline \multicolumn{1}{|c|}{ CRITERIOS } & CARACTERÍSTICAS \\
\hline $\begin{array}{l}\text { Carga asintomática } \\
\text { mMRC } 2=\text { camina más lento que las personas } \\
\text { de la misma edad, debido a la disnea o tiene } \\
\text { que detenerse para respirar cuando camina a } \\
\text { su propio ritmo. }\end{array}$ & $\begin{array}{l}\text { Inferior (mMRC }<2 \text { o } \\
\text { puntuación CAT }<10 \text { ). }\end{array}$ \\
$\begin{array}{l}\text { CAT 10 = los síntomas de la EPOC tienen un } \\
\text { impacto bajo a medio en la vida del paciente. }\end{array}$ & $\begin{array}{l}\text { Más alto (mMRC } \geq 2 \text { o } \\
\text { puntuación CAT } \geq 10) .\end{array}$ \\
\hline & $\begin{array}{l}0 \text { exacerbaciones. } \\
1 \quad \text { exacerbación sin } \\
\text { ingreso hospitalario. } \\
\geq 1 \quad \text { exacerbación con } \\
\text { Historia de exacerbaciones }\end{array}$ \\
\begin{tabular}{l}
$\geq 2$ exacerbaciones. \\
\hline
\end{tabular}
\end{tabular}




\section{Fórmula}

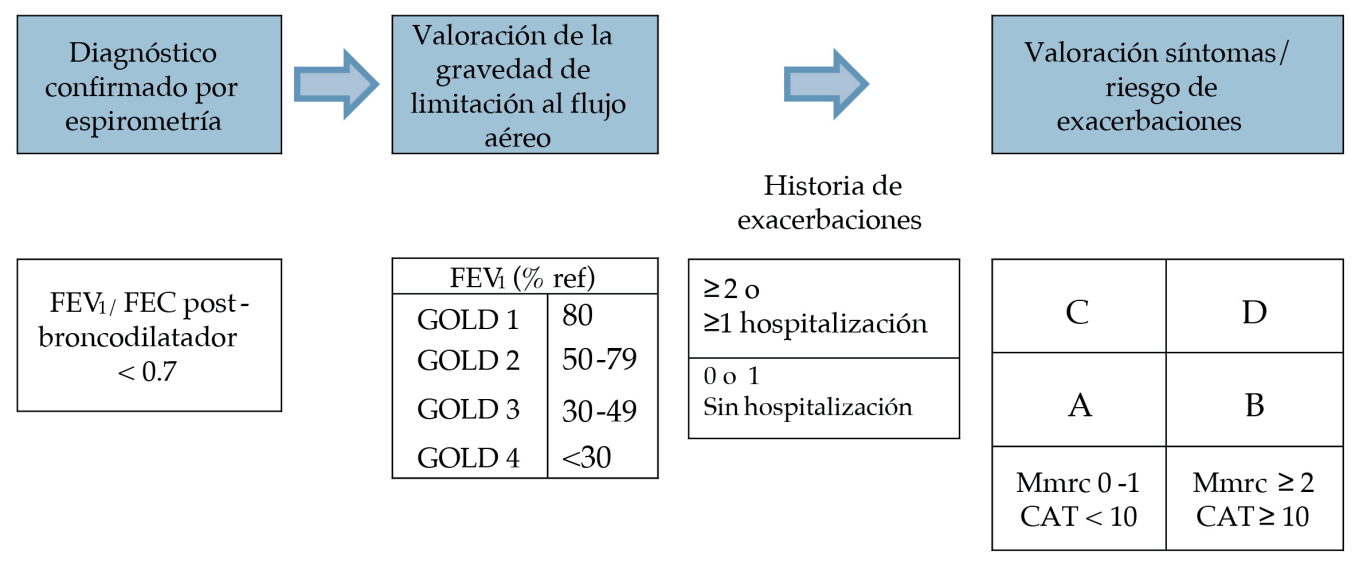

\section{Interpretación}

GOLD 1-4 se refiere al grado de obstrucción del flujo de aire, y GOLD AD son los grupos en los que se basan las recomendaciones de tratamiento.

\begin{tabular}{|c|l|l|l|}
\hline $\begin{array}{c}\text { GRUPO } \\
\text { GOLD }\end{array}$ & RIESGO & $\begin{array}{c}\text { MORTALIDAD } \\
\text { A 3 ANNOS }\end{array}$ & \multicolumn{1}{|c|}{$\begin{array}{l}\text { RECOMENDACIÓN } \\
\text { DE TRATAMIENTO } \\
\text { FARMACOLÓGICO }\end{array}$} \\
\hline A & $\begin{array}{l}\text { Bajo riesgo, } \\
\text { baja carga de } \\
\text { síntomas. }\end{array}$ & $3.8 \%$ & $\begin{array}{l}\text { Broncodilatador (acción } \\
\text { larga o corta). }\end{array}$ \\
\hline B & $\begin{array}{l}\text { Bajo riesgo, } \\
\text { mayor carga } \\
\text { sintomática. }\end{array}$ & $10.6 \%$ & $\begin{array}{l}\text { Broncodilatador de acción } \\
\text { prolongada (LABA) o } \\
\text { antagonista de la metacolina } \\
\text { de acción prolongada } \\
\text { (LAMA). }\end{array}$ \\
\hline C & $\begin{array}{l}\text { Alto riesgo, } \\
\text { baja carga } \\
\text { sintomática. }\end{array}$ & $8.2 \%$ & $\begin{array}{l}\text { LAMA (o LAMA + LABA, o } \\
\text { LABA+ ICS (corticosteroides } \\
\text { inhalados) son } \\
\text { exacerbaciones frecuentes). }\end{array}$ \\
\hline D & $\begin{array}{l}\text { Alto riesgo, } \\
\text { mayor carga } \\
\text { sintomática. }\end{array}$ & $20.1 \%$ & $\begin{array}{l}\text { LAMA y LABA, más } \\
\text { corticosteroides inhalados } \\
\text { (ICS) si exacerbaciones } \\
\text { frecuentes. }\end{array}$ \\
\hline LAMA: and
\end{tabular}

LAMA: antimuscarínicos de acción prolongada; LABA: beta-2 agonistas de larga duración; ICS: corticoesteroides inhalados. 


\section{Bibliografía}

\section{Original/Primaria}

Iniciativa global para la enfermedad pulmonar obstructiva crónica. Estrategia Global para el Diagnóstico, Manejo de la Enfermedad Pulmonar Obstructiva Crónica, Informe del Taller NHLBI 2003.

\section{Validación}

Petsonk EL, Hnizdo E, Attfield M. Definición de COPD GOLD etapa I. Thorax [Internet]; 2007 [citado marzo 2019]; 62 (12): 1107-1108. Disponible en: https: / / www.ncbi.nlm.nih.gov/ pmc/articles/ PMC2094288/. 


\section{CRISIS ASMÁTICA}

\section{EVALUACIÓN DE LAS CRISIS DE ASMA EN ADULTO (ALERTA 2-2010)}

\section{Utilidad}

Se utiliza para la evaluación de la gravedad de la exacerbación del asma.

\section{Parámetros}

\begin{tabular}{|c|c|c|c|c|}
\hline & \multicolumn{4}{|c|}{ CRISIS } \\
\hline $\begin{array}{c}\text { CRITE- } \\
\text { RIO }\end{array}$ & LEVE & $\begin{array}{c}\text { MODERA- } \\
\text { DA-IN- } \\
\text { TENSA }\end{array}$ & $\begin{array}{l}\text { PARADA } \\
\text { RESPIRATO- } \\
\text { RIA INMINEN- } \\
\text { TE }\end{array}$ & $\begin{array}{l}\text { INTERPRETA- } \\
\text { CIÓN }\end{array}$ \\
\hline Disnea & Leve & $\begin{array}{l}\text { Moderada, } \\
\text { intensa }\end{array}$ & Muy intensa & $\begin{array}{l}\text { Presenta en casi } \\
\text { todos los pacien- } \\
\text { tes. } \\
\text { Difíciles de cuan- } \\
\text { tificar. } \\
\text { Correlación po- } \\
\text { bre con la obs- } \\
\text { trucción. }\end{array}$ \\
\hline Habla & Párrafos & $\begin{array}{l}\text { Frases/ } \\
\text { palabra }\end{array}$ & & $\begin{array}{l}\text { Difícil de medir } \\
\text { Pobre correlación } \\
\text { con la obstruc- } \\
\text { ción. }\end{array}$ \\
\hline FR (RPM) & $\begin{array}{c}\text { Aumen- } \\
\text { tada }\end{array}$ & $>20-30$ & & $\begin{array}{lr}\text { Menos } 10 \% \text { de } \\
\text { asmáticos } \\
\text { presentan } \\
\text { trecuencia } \\
\text { ratoria }<25 \text { respi- } \\
\text { rato }\end{array}$ \\
\hline FC (LPM) & $>100$ & $>100-120$ & Bradicardia & $\begin{array}{l}\text { No más del } 15 \% \\
\text { con crisis grave } \\
\text { presenta una FC } \\
>120 \text {. }\end{array}$ \\
\hline Triaje alto & Ausente & Presente & $\begin{array}{c}\text { Movimiento } \\
\text { paradójico o } \\
\text { incoordinación } \\
\text { toracoabdomi- } \\
\text { nal }\end{array}$ & $\begin{array}{l}\text { indicador de obs- } \\
\text { trucción grave de } \\
\text { la via aerea y/o } \\
\text { fatiga diafragmá- } \\
\text { tica. }\end{array}$ \\
\hline
\end{tabular}




\begin{tabular}{|c|c|c|c|c|}
\hline $\begin{array}{l}\text { Sibilan- } \\
\text { cias }\end{array}$ & Presentes & Presentes & $\begin{array}{l}\text { Silencio auscula- } \\
\text { torio }\end{array}$ & $\begin{array}{l}\text { Presentes en casi } \\
\text { todos los pacien- } \\
\text { tes. } \\
\text { Pobre correlación } \\
\text { con la obstruc- } \\
\text { ción. }\end{array}$ \\
\hline $\begin{array}{l}\text { Concien- } \\
\text { cia }\end{array}$ & Normal & Normal & Disminuida & Signo tardío. \\
\hline $\begin{array}{l}\text { Pulso } \\
\text { paradójico }\end{array}$ & Ausente & $\begin{array}{l}>10-25 \\
\mathrm{mmHg}\end{array}$ & $\begin{array}{c}\text { Ausencia (fatiga } \\
\text { muscular) }\end{array}$ & $\begin{array}{l}\text { Difícil de medir y } \\
\text { poco fiable. }\end{array}$ \\
\hline $\mathrm{FEV}_{1} / \mathrm{PEF}$ & $>70 \%$ & $<70-50 \%$ & & $\begin{array}{l}\text { Medida objetiva } \\
\text { de la obstrucción } \\
\text { de la vía aérea y } \\
\text { respuesta al trata- } \\
\text { miento. }\end{array}$ \\
\hline $\mathrm{SAT} \mathrm{O}_{2}$ & $>95 \%$ & $<95 \%$ & $<90 \%$ & $\begin{array}{l}\text { Determinar el ni- } \\
\text { vel de hipoxemia } \\
\text { Predictor pobre } \\
\text { de la respuesta al } \\
\text { tratamiento. }\end{array}$ \\
\hline $\mathrm{PAO}_{2}$ & Normal & $80-60$ & $<60$ & $\begin{array}{l}\text { Pobre relación } \\
\text { con el nivel de } \\
\text { obstrucción. }\end{array}$ \\
\hline $\mathrm{PACO}_{2}$ & $<40$ & $>40$ & $>40$ & $\begin{array}{l}\text { Signo tardío, po- } \\
\text { bre correlación } \\
\text { con el nivel de } \\
\text { obstrucción. }\end{array}$ \\
\hline
\end{tabular}

FEM: flujo espiratorio máximo; FEV1: volumen espiratorio máximo en el primer segundo; LPM: latidos por minuto; PaCO2: presión arterial de anhídrido carbónico; PaO2: presión arterial de oxígeno; RPM: respiraciones por minuto.

\section{Bibliografía}

\section{Original/Primaria}

1. Patología respiratoria aguda Protocolos de manejo, segunda edición (pp.102). 


\section{CÁLCULO DEL GRADIENTE ALVEOLO-ARTERIAL DE O2}

\section{Utilidad}

Es un indicador de la capacidad pulmonar como intercambiador de gases. Mide la eficacia de la oxigenación de oxígeno.

\section{Descripción}

Se usa en pacientes con hipoxia inexplicable y pacientes con hipoxia superior al grado de su enfermedad clínica.

El gradiente se ve alterado en trastornos de ventilación/perfusión o de la difusión, pero no en hipo o hiperventilación.

\section{Parámetros}

\section{CRITERIOS}

\section{Presión atmosférica}

Use $760 \mathrm{~mm} \mathrm{Hg}$ si está a nivel del mar.

\begin{tabular}{l}
$\mathrm{PaO}_{2}(\mathrm{mmHg})$ \\
\hline $\mathrm{FiO}_{2}(\%)$ \\
\hline $\mathrm{PaCO}_{2}(\mathrm{mmHg})$ \\
\hline
\end{tabular}

\section{Edad}

PaCO2: presión arterial de anhídrido carbónico; PaO2: presión arterial de oxígeno;FIO2: fracción inspirada de oxigeno.

\section{Fórmula}

$$
\text { Gradiente A-a } \left.\mathrm{O}^{2}=\left[\left(\mathrm{Fio}_{2}\right) \text { x (presión atmos férica-presión } \mathrm{H}_{2} \mathrm{O}\right)-\left(\frac{\mathrm{PaCO}_{2}}{0.8}\right)-\mathrm{PaO}_{2}\right]
$$

\section{Interpretación}

El rango de normalidad para el gradiente A-a varía con la edad, y oscila entre 7-20 mmHg. Un valor inferior a $20 \mathrm{mmHg}$ es indicativo de normalidad o causa extrapulmonar de trastorno ventilatorio. Mientras que un valor por encima de $20 \mathrm{mmHg}$ indica afectación pulmonar.

$$
\text { Gradiente esperado para la edad }=\left(\frac{e d a d}{4}\right)+4
$$




\section{Bibliografía}

\section{Original/Primaria}

1. Helmholz HF Jr. La ecuación de aire alveolar abreviada. Chest [Internet]. Junio de 1979 [citado marzo 2019]; 75 (6): 748. Disponible en: https:/ / www.ncbi.nlm.nih.gov/ pubmed / 436542.

\section{Validación}

1. McFarlane MJ, Imperiale TF. Uso del gradiente de oxígeno alveolararterial en el diagnóstico de embolia pulmonar. Am J Med [Internet]. 1994 Enero [citado marzo 2019]; 96 (1): 57-62. Disponible en: https: / / www.ncbi.nlm.nih.gov/pubmed/8304364. 


\section{EMBOLIA PULMONAR}

\section{CRITERIOS DE WELLS}

\section{Utilidad}

Se usan como predictor del riesgo de embolia pulmonar.

\section{Descripción}

- Wells no está destinado a diagnosticar la EP, sino a orientar el trabajo mediante la predicción de la probabilidad de la EP previa a la prueba y las pruebas apropiadas para descartar el diagnóstico.

\section{Parámetros}

\begin{tabular}{|l|c|}
\hline CRITERIOS & PUNTAJE \\
\hline Signos y síntomas de TVP & 3 \\
\hline $\begin{array}{l}\text { Embolia pulmonar es el diagnóstico número 1 o el más } \\
\text { probable. }\end{array}$ & 3 \\
\hline Frecuencia cardíaca > 100 & 1.5 \\
\hline $\begin{array}{l}\text { Inmovilización al menos } 3 \text { días o cirugía en las 4 } \\
\text { semanas previas. }\end{array}$ & 1.5 \\
\hline Diagnóstico previo de EP o TVP & 1.5 \\
\hline Hemoptisis & 1 \\
\hline Cáncer con tratamiento los últimos 6 meses o paliativo. & 1 \\
\hline
\end{tabular}

EP: embolia pulmonar, TVP: trombosis venosa profunda.

\section{Interpretación}

\begin{tabular}{|cc|}
\hline PUNTOS & PREVALENCIA DE TVP \\
\hline & Modelo de tres niveles \\
\hline $0-1$ & Riesgo bajo \\
\hline $2-6$ & Riesgo moderado \\
\hline$>6$ & Alto riesgo \\
\hline
\end{tabular}




\section{Original/Primaria}

1. Wells PS, Anderson DR, Rodger M, Stiell I, Dreyer JF, Barnes D,et al. Excluyendo la embolia pulmonar en la cabecera sin imágenes de diagnóstico: manejo de pacientes con sospecha de embolia pulmonar que se presentan en el servicio de urgencias mediante el uso de un modelo clínico simple y dímero d. Ann Intern Med [Internet]. 2001 [citado marzo 2019];135 (2): 98-107. Disponible en : https:/ / www. ncbi.nlm.nih.gov/pubmed/11453709.

\section{Validación}

1. Wolf SJ, McCubbin TR, Feldhaus KM, Faragher JP, Adcock DM. Validación prospectiva de los criterios de Wells en la evaluación de pacientes con sospecha de embolia pulmonar. Ann Emerg Med [Internet]. 2004 [citado marzo 2019]; 44 (5): 503-10. Disponible en: https: / / www.ncbi.nlm.nih.gov/pubmed/15520710.

2. van Belle A, Buller HR, Huisman MV, et al. Eficacia del manejo de la sospecha de embolia pulmonar mediante un algoritmo que combina la probabilidad clínica, la prueba del dímero D y la tomografía computarizada. JAMA [Internet]. 2006 [citado marzo 2019]; 295 (2): 172-179. Disponible en: https://www.ncbi.nlm.nih.gov/ pubmed / 15520710 . 


\section{ESCALA DE GINEBRA}

\section{Utilidad}

Es una escala de previsión clínica, utilizada para determinar la probabilidad de troboembolismo pulmonar.

\section{Descripción}

- Puede estratificar a los pacientes en riesgo bajo, intermedio o alto, basado solo en la historia y el examen físico.

\section{Parámetros}

$\left.\begin{array}{|l|c|}\hline \multicolumn{1}{|c|}{\text { CRITERIOS }} & \text { PUNTAJE } \\ \hline \text { Edad > 65 años } & 1 \\ \hline \text { TVP anterior } & 3 \\ \hline \begin{array}{l}\text { Cirugía (bajo anestesia general) o fractura de miembro } \\ \text { inferior en el último mes. }\end{array} & 2 \\ \hline \text { Condición maligna activa } & 2 \\ \text { Condición maligna sólida o hematológica, actualmente } \\ \text { activa o considerada curada }<1 \text { año. }\end{array}\right)$

EP: embolia pulmonar, TVP: trombosis venosa profunda. 


\section{Interpretación}

\begin{tabular}{|cl|}
\hline PUNTOS & \multicolumn{1}{c|}{ PROBABILIDAD DE TVP } \\
\hline $0-5$ & Riesgo bajo \\
\hline $5-8$ & Riesgo moderado \\
\hline$>9$ & Alto riesgo \\
\hline
\end{tabular}

\section{Bibliografía}

\section{Original / Primaria}

1. Le Gal G, Righini M, Roy PM, Sanchez O, Aujesky D, Bounameaux H, Perrier A. Predicción de embolia pulmonar en el servicio de urgencias: la puntuación revisada de Ginebra. Ann Intern Med [Internet]. 2006 [citado marzo 2019]; 144 (3): 165-71. Disponible en: https:/ / www. mdcalc.com/geneva-score-revised-pulmonary-embolism\# evidence.

\section{Validación}

1. CerianiE,CombescureC, LeGalG, NendazM,Perneger T, Bounameaux $H$. Reglas de predicción clínica para la embolia pulmonar: una revisión sistemática y un metanálisis. J Thromb Haemost [Internet]. 2010 mayo [citado marzo 2019]; 8 (5): 957-70. Disponible en: https:// www.ncbi. nlm.nih.gov/pubmed/20149072. 


\section{CRITERIOS PERC PARA EMBOLIA PULMONAR}

\section{Utilidad}

Los criterios PERC (Pulmonary Embolism Rule Out Criteria), son un conjunto de criterios orientados a detectar un posible Embolismo Pulmonar en personas con sospecha.

\section{Descripción}

- PERC se aplica únicamente a pacientes con riesgo de embolia pulmonar.

- Los criterios PERC se utilizan para descartar la embolia pulmonar y evitar pruebas adicionales de embolia pulmonar en pacientes considerados de bajo riesgo.

\section{Parámetros}

\begin{tabular}{|l|c|}
\hline CRITERIOS & PUNTAJE \\
\hline Edad $\geq 50$ años & 1 \\
\hline Pulso $\geq 100$ & 1 \\
\hline $\mathrm{SaO}_{2}<95 \%$ & 1 \\
\hline Hinchazón unilateral de la pierna & 1 \\
\hline Hemoptisis & 1 \\
\hline Cirugía reciente o trauma & 1 \\
\hline $\begin{array}{l}\text { Embolismo pulmonar o trombosis venosa profunda } \\
\text { anterior. }\end{array}$ & 1 \\
\hline $\begin{array}{l}\text { Uso hormonal } \\
\begin{array}{l}\text { Anticonceptivos orales, reemplazo hormonal o uso de } \\
\text { hormonas estrogénicas en hombres o mujeres. }\end{array}\end{array}$ \\
\hline
\end{tabular}

\section{Interpretación}

Si ningún criterio es positivo, el riesgo de que la etiología sea por embolia, es menor de $15 \%$. 


\section{Bibliografía}

\section{Original/Primaria}

Kline JA, Mirchell AM, Kabrhel C, Richman PB, Courtney DM. Criterios clínicos para prevenir pruebas diagnósticas innecesarias en pacientes de urgencias con sospecha de embolia pulmonar. J Thromb Haemost [Internet] 2004 [citado marzo 2019];2:1247-55. Disponible en: https:/ / www.ncbi.nlm.nih.gov/pubmed/15304025.

\section{Validación}

Kline JA, Courtney DM, Kabrhel C, Moore CL, Smithline HA, Plewa MC, et al. Prospectiva evaluación multicéntrica de los criterios de descarte de embolia pulmonar. J Thromb Haemost [Internet]. 2008 [citado marzo 2019] ;6(5):772-80. Disponible en: https://www.ncbi.nlm.nih.gov/ pubmed/18318689. 


\section{TROMBOSIS VENOSA PROFUNDA}

\section{CRITERIOS DE WELLS PARA TROMBOSIS VENOSA PROFUNDA} (TVP)

\section{Utilidad}

Se utiliza para estimar la probabilidad de TVP en un paciente.

\section{Descripción}

- Los criterios de la TVP de Wells solo deben aplicarse después de realizar un historial detallado y un examen físico completo.

- Los criterios de la TVP de Wells solo deben aplicarse a aquellos pacientes que se consideran en riesgo de TVP. Si no hay preocupación por la TVP, no hay necesidad de estratificar el riesgo.

\section{Parámetros}

\begin{tabular}{|l|c|}
\hline CRITERIOS & PUNTAJE \\
\hline Cáncer activo. & 1 \\
\hline $\begin{array}{l}\text { Parálisis o inmovilización reciente de un miembro } \\
\text { inferior. }\end{array}$ & 1 \\
\hline $\begin{array}{l}\text { Encamamiento recientemente de más de 3 días o } \\
\text { cirugía mayor dentro de las } 12 \text { últimas semanas. }\end{array}$ & 1 \\
\hline $\begin{array}{l}\text { Hinchazón de la pantorrilla> } 3 \text { cm en comparación } \\
\text { con la otra pierna. }\end{array}$ & 1 \\
\hline Colateral (no varicosa): venas superficiales presentes. & 1 \\
\hline Pierna entera hinchada. & 1 \\
\hline $\begin{array}{l}\text { Sensibilidad localizada a lo largo del sistema venoso } \\
\text { profundo. }\end{array}$ & 1 \\
\hline Edema con fóvea, confinado a pierna sintomática. & -2 \\
\hline TVP previamente documentado. & 1 \\
\hline $\begin{array}{l}\text { Diagnóstico alternativo a la TVP según sea probable o } \\
\text { más probable. }\end{array}$ & 1 \\
\hline
\end{tabular}




\section{Interpretación}

\section{PUNTOS RIESGO}

\begin{tabular}{|lll|}
\hline $\mathbf{1 0}$ & Baja/ poco probable & $5 \%$ \\
\hline $\mathbf{1 - 2}$ & Moderar & $17 \%$ \\
\hline $\mathbf{3}$ & Alto & $17-53 \%$ \\
\hline
\end{tabular}

\section{Bibliografía}

\section{Original/Primaria}

1. Wells PS, Hirsh J, Anderson DR, et al. Precisión de la valoración clínica de la trombosis venosa profunda. Lancet [Internet]. 1995 [citado marzo 2019]; 345 (8961): 1326-30. Disponible en: https: / www.ncbi.nlm.nih. gov / pubmed / 7752753 . 


\section{FIBROSIS PULMONAR}

SCORE DU BOIS PARA MORTALIDAD POR FIBROSIS PULMONAR IDIOPÁTICA

\section{Utilidad}

Determina la mortalidad a 1 año en pacientes con FPI (Fibrosis Pulmonar Idiopática) utilizando pruebas de función pulmonar e indicadores clínicos.

\section{Descripción}

Las puntuaciones más altas corresponden a una mayor necesidad de transplante y otros tratamientos.

\section{Parámetros}

\begin{tabular}{|l|c|}
\hline CRITERIO & PUNTAJE \\
\hline Edad & 0 \\
$<60$ años & 4 \\
$60-69$ años & 8 \\
$\geq 70$ años & 14 \\
\hline Hospitalización respiratoria en los últimos 6 meses. & \\
\hline Línea de base pronosticada de capacidad vital forzada & \\
(FVC). & 0 \\
$\geq 80 \%$ & 8 \\
$66-79 \%$ & 13 \\
$51-75 \%$ & 18 \\
$\geq 50 \%$ & 21 \\
\hline Cambio de 24 semanas en FVC predicho. & 10 \\
$\leq-10 \%$ & 0 \\
\hline$-5 \%--9.9 \%$ & \\
$\geq-4.9 \%$ & \\
\hline
\end{tabular}

FVC: capacidad vital forzada. 


\section{Interpretación}

\begin{tabular}{|c|c|}
\hline PUNTOS & PROBABILIDAD DE MUERTE A 1 AÑO \\
\hline $\mathbf{0 - 4}$ & $<2 \%$ \\
\hline $\mathbf{8 - 1 4}$ & $2-5 \%$ \\
\hline $\mathbf{1 6 - 2 1}$ & $5-10 \%$ \\
\hline $\mathbf{2 2 - 2 9}$ & $10-20 \%$ \\
\hline $\mathbf{3 0 - 3 3}$ & $20-30 \%$ \\
\hline $\mathbf{3 4 - 3 7}$ & $30-40 \%$ \\
\hline $\mathbf{3 8 - 4 0}$ & $40-50 \%$ \\
\hline $\mathbf{4 1 - 4 3}$ & $50-60 \%$ \\
\hline $\mathbf{4 4 - 4 5}$ & $60-70 \%$ \\
\hline $\mathbf{4 7 - 4 9}$ & $70-80 \%$ \\
\hline $\mathbf{7 5 0}$ & $>80 \%$ \\
\hline
\end{tabular}

\section{Bibliografía}

\section{Original/Primaria}

1. du Bois RM, Weycker D, Albera C, Bradford WZ, Costabel U, Kartashov A, et al. Determinación del riesgo individual de mortalidad en pacientes con fibrosis pulmonar idiopática. Am J Respir Crit Care Med [Internet].2011 [citado 27 marzo 2019]; 184: 459-66. Disponible en: https: / / www.ncbi.nlm.nih.gov/ pubmed / 21616999.

\section{Validación}

1. du Bois RM, Weycker D, Albera C, Bradford WZ, Costabel U, Kartashov A, et al. Capacidad vital forzada en pacientes con fibrosis pulmonar idiopática: propiedades de prueba y mínima diferencia clínicamente importante. Am J Respir Crit Care Med. 2011 [citado marzo 2019]. Disponible en: https: / / www.ncbi.nlm.nih.gov/ pubmed/ 21940789.

2. Kolb M, Collard HR. Staging of idiopathic pulmonary fibrosis: past, present and future. Eur Respir Rev [Internet]. 2014 [citado marzo 2019];23:220-224. Disponibleen: https: / / err.ersjournals.com/content/ errev/23/132/220.full.pdf. 


\section{DERRAME PLEURAL}

\section{CRITERIOS LIGHT PARA DERRAMES EXUDATIVOS}

\section{Utilidad}

Ayuda a determinar si el líquido pleural es exudado o trasudado.

\section{Descripción}

- Los criterios de Light son más sensibles que específicos para derrames exudativos.

\section{Parámetros}

\begin{tabular}{|c|c|c|}
\hline CRITERIOS & TRASUDADO & EXUDADO \\
\hline $\begin{array}{c}\text { Relación LDH } \\
\text { pleural/suero }\end{array}$ & $<0.6$ & $>0.6$ \\
\hline $\begin{array}{c}\text { Relación proteínas } \\
\text { pleural/suero }\end{array}$ & $<0.5$ & $>0.5$ \\
\hline $\begin{array}{c}\text { LDH en líquido } \\
\text { pleural }\end{array}$ & $\begin{array}{c}\leq \text { a } 2 / 3 \text { del límite } \\
\text { superior normal de } \\
\text { LDH sérica. }\end{array}$ & $\begin{array}{c}>\text { de } 2 / 3 \text { del límite } \\
\text { superior del valor } \\
\text { normal sérico. }\end{array}$ \\
\hline
\end{tabular}




\section{Interpretación}

\begin{tabular}{|l|l|}
\hline EXUDATIVO & TRASUDATIVO \\
\hline Malignidad & \\
SDRA & Insuficiencia cardíaca \\
Pancreatitis & Atelectasia \\
Granulomatosis eosinofílica con & Fuga de LCR en el espacio pleural \\
poliangitis & Hidrotórax hepático \\
Granulomatosis con poliangeitis & Hipoalbuminemia \\
Lupus & Síndrome nefrótico \\
Absceso pulmonar & Diálisis peritoneal \\
Quilotórax & \\
Sarcoidiosis & \\
Hipotiroidismo & \\
\hline
\end{tabular}

\section{Bibliografía}

\section{Original/Primaria}

1. Light RW, et. Alabama. Derrame pleural: la separación diagnóstica de transudados y exudados. Ann Intern Med [Internet]. 1972 [citado 27 marzo 2019]; 77: 507-513. doi: 10.7326 / 0003-4819-77-4-507. Disponible en: https://annals.org/aim/article-abstract/686932/ pleural-effusions-diagnostic-separation-transudates-exudates.

\section{Validación}

1. Light RW, Erozan YS, Ball WC. Células en el líquido pleural. Su valor en el diagnóstico diferencial. Arch Intern Med [Internet]. 1973 [citado marzo 2019];132(6):854-60. Disponible en: https:/ / www.ncbi.nlm.nih. gov/pubmed / 4757257. 


\section{INTUBACIÓN ENDOTRAQUEAL}

\section{CLASIFICACIÓN MODIFICADA DE MALLAMPATI}

\section{Utilidad}

Estratifica la dificultad de la intubación endotraqueal basada en características anatómicas.

\section{Instrucciones}

Coloque al paciente sentado en posición vertical y dirija al paciente a que abra la boca y sobresalga la lengua por completo.

\section{Parámetros}

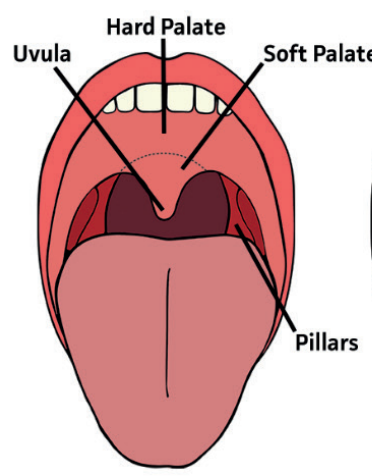

Class I

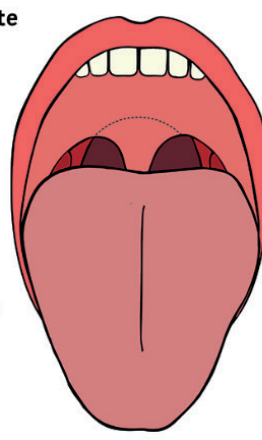

Class II

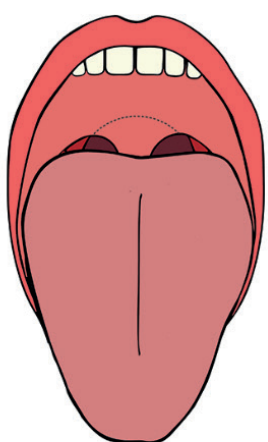

Class III

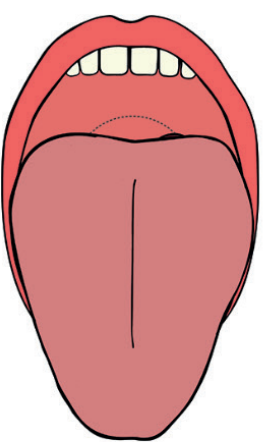

Class IV

\begin{tabular}{|l|l|}
\hline CLASE I & Paladar blando, úvula, fauces, pilares visibles. \\
\hline CLASE II & Paladar blando, úvula, fauces visibles. \\
\hline CLASE III & Paladar blando, base de la úvula visible. \\
\hline CLASE IV & Solo el paladar duro es visible. \\
\hline
\end{tabular}

\section{Interpretación}

\begin{tabular}{|l|l|}
\hline CLASE I & No dificultad adicional. \\
\hline CLASE II & No dificultad adicional. \\
\hline
\end{tabular}




\begin{tabular}{|l|l|}
\hline CLASE III & $\begin{array}{l}\text { Dificultad moderada, área difícil prevista por Mallampati, } \\
\text { considere estrategias de apoyo. }\end{array}$ \\
\hline CLASE IV & $\begin{array}{l}\text { Dificultad severa, área difícil prevista por Mallampati, } \\
\text { considere estrategias de apoyo. }\end{array}$ \\
\hline
\end{tabular}

\section{Bibliografía}

\section{Original/Primaria}

1. Mallampati SR, Gatt SP, Gugino LD, et al. Un signo clínico para predecir la intubación traqueal difícil: un estudio prospectivo. Can Anaesth Soc J [Internet]. 1985 [citado 27 marzo 2019]; 32 (4): 429-34. Disponible en: https: / / www.ncbi.nlm.nih.gov/pubmed/ 4027773.

\section{Validación}

1. Lee A, Fan LT, Gin T, Karmakar MK, Ngan Kee WD. Una revisión sistemática (meta-análisis) de la precisión de las pruebas de Mallampati para predecir la vía aérea difícil.Anaesth Analg [Internet]. 2006 [citado marzo 2019]. 102 (6): 1867-78. Disponible en: https:/ / www.ncbi.nlm. nih.gov/pubmed/16717341.

2. Samsoon GL, Joven JR. Intubación traqueal difícil: un estudio retrospectivo. Anaesthesia [Internet]. 1987 [citado marzo 2019]. 42 (5): 487-90. Disponible en: https://www.ncbi.nlm.nih.gov/ pubmed/3592174.

3. Apfelbaum JL, Hagberg CA, Caplan RA, et al. Guías de práctica para el manejo de la vía aérea difícil: un informe actualizado del Equipo de Trabajo de la Sociedad Americana de Anestesiología para el Manejo de la Vía Aérea Difícil. Anestesiología. 2013;118(2):25170. Disponible en: https://anesthesiology.pubs.asahq.org/article. aspx?articleid=1918684. 
CÁCULOSY

PROHÓSTICAS

EN MEDICINA INTERNA 

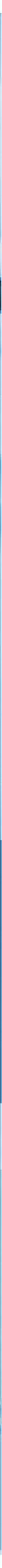


\section{Utilidad}

La osmolaridad es la medición de la concentración de solutos, definida como el número de osmoles (Osm) de un soluto por litro (L) de solución (Osm/L).

\section{Parámetros}

Sodio

BUN

Glucosa

Fórmula

\section{Interpretación}

$$
\text { Osmolaridad }=\mathrm{Na} \times 2+\frac{\operatorname{Urea}\left(\frac{m g}{d l}\right)}{3}+\frac{\operatorname{Glucemia}\left(\frac{m g}{d l}\right)}{18}
$$

Osmolalidad sérica normal $=285-295 \mathrm{mOsm} / \mathrm{kg}$

\section{Bibliografía}

\section{Original/Primaria}

1. Purssell RA, Pudek M, Brubacher J, Abu-Laban RB. Derivación y validación de una fórmula para calcular la contribución del etanol a la brecha osmolal. Ann Emerg Med [Internet] 2001 [citado marzo 2019];38(6):653-9. Disponible en: https://www.sciencedirect.com/ science/ article/ pii/S0196064401166667.

\section{Validación}

1. Siervo M, Bunn D, Prado CM, Hooper L. Precisión de las ecuaciones de predicción para la osmolaridad sérica en personas mayores frágiles con y sin diabetes. Am J Clin Nutr [Internet]. 2014 [citado marzo 2019]; 100 (3): 867-876. Disponible en: https: / / www.ncbi.nlm.nih.gov / pmc/ articles/PMC4135495/.

2. Glasser L, Sternglanz PD, Combie J, Robinson A. Osmolalidad sérica y su aplicabilidad a la sobredosis de drogas. A.m. J. Clin. Camino [Internet]. 1973 [citado marzo 2019];60:695. Disponible en: https:// www.ncbi.nlm.nih.gov/pubmed/4751712. 


\section{ANIÓN GAP EN SUERO}

\section{Utilidad}

El anión GAP o brecha aniónica es la diferencia entre los aniones y cationes medidos en suero. Esta fórmula orienta acerca de la posible causa de la acidosis metabólica.

Fórmula

Anion $\mathrm{GAP}=\mathrm{Na}-\left(\mathrm{Cl}+\mathrm{HCO}_{3}\right)$

Interpretación

Valor normal $=10 \pm 2$

\begin{tabular}{|l|l|l|l|}
\hline & $\begin{array}{l}\text { Acidosis metabólica con } \\
\text { Anión GAP elevado }\end{array}$ & & $\begin{array}{l}\text { Acidosis sin Anión GAP } \\
\text { elevado }\end{array}$ \\
\hline M & Metanol & H & Hiperalimentación \\
U & Uremia & A & Acetazoamida \\
D & Cetoacidosis diabética & R & Acidosis tubular renal \\
P & Paraldehido & D & Diarrea \\
I & Isoniacida & U & Derivación uretero-pélvica \\
L & Acidosis láctica & $\mathbf{P}$ & Posthipocapnia \\
E & Etanol & S & Espironolactona \\
R & Insuficiencia renal & & \\
S & Salicilatos & \\
\hline
\end{tabular}




\section{Bibliografía}

\section{Original/Primaria}

1. Oh MS, Carroll HJ. La brecha aniónica. N. Engl. J. Med [Internet]. 1977 [citado marzo 2019]; 297 (15): 814-7. Disponible en: https:/ /www. ncbi.nlm.nih.gov/pubmed/895822.

\section{Validación}

1. Criner GJ. Alteración metabólica de ácido-base y electrolitos. En: Guía de estudio de cuidados críticos: Texto y revisión. $2^{\mathrm{a}}$ ed. Filadelfia, PA: Springer; 2010: 696.

2. Berend K, de Vries AP, Gans RO. Enfoque fisiológico para la evaluación de alteraciones ácido-base. N. Engl. J. Med [Internet]. 20149 de octubre [citado marzo 2019]; 371 (15): 1434-45. Disponible en: https:/ / www. ncbi.nlm.nih.gov/pubmed/25295502. 


\section{DÉFICIT DE BICARBONATO}

\section{Utilidad}

Calcula el déficit de bicarbonato corporal.

\section{Fórmula}

Déficit de $\mathrm{HCO}_{3}=$ (Bicarbonato normal-Bicarbonato medido) $x$ peso en $\mathrm{kg} x 0.4$

\section{Alternativa:}

Déficit de $\mathrm{HCO}_{3}=($ Exceso de base (EB ) x peso en $\mathrm{kg} \times 0.3$

Déficit de HCO3 dividir para 8.4 (meq presentes en 1 ampolla de $10 \mathrm{ml}$ de bicarbonato), ese resultado será el número de ampollas de HCO3. De éste total, administrar solo el 50\% intravenoso en 1-2 horas. Realizar un control gasométrico en 60 minutos posterior a la infusión de bicarbonato.

\section{Bibliografía}

\section{Original/Primaria}

1. Kurtz I. Estudios de caso ácido-base. $2^{a}$ ed. Trafford Publishing (2004); 68: 150. 


\section{FRACCIÓN DE EXCRECIÓN DE SODIO (FENA)}

\section{Utilidad}

Determina si la insuficiencia renal aguda se debe a patología pre-renal, renal o post-renal.

\section{Descripción}

- Usar en pacientes con oliguria y/o lesión renal aguda de etiología poco clara.

- No usar en paciente que usan diuréticos o con enfermedad renal crónica.

\section{Parámetros}

Creatinina en suero:

Creatinina en orina:

$$
\begin{aligned}
& \mathrm{S}_{\mathrm{Cr}} \\
& \mathrm{O}_{\mathrm{Cr}} \\
& \mathrm{S}_{\mathrm{Na}} \\
& \mathrm{O}_{\mathrm{Na}}
\end{aligned}
$$

0,7-1,3 mg/ dl 20-370 mg/dl $136-145 \mathrm{mEq} / \mathrm{L}$ $100-260 \mathrm{mEq} / \mathrm{L}$

\section{Fórmula}

$$
\boldsymbol{F E N a}=\frac{O_{\mathrm{Na}} \times S_{\mathrm{Cr}}}{S_{\mathrm{Na}} \times O_{\mathrm{Cr}}} \times 100
$$

\section{Interpretación}

\begin{tabular}{|l|l|l|}
\hline Insuficiencia renal & FENa & CAUSAS \\
\hline Pre-renal & $<1 \%$ & $\begin{array}{l}\text { Hipovolemia, insuficiencia cardíaca, } \\
\text { estenosis de la arteria renal, Sepsis, etc. }\end{array}$ \\
\hline Renal & $>1 \%$ & $\begin{array}{l}\text { Necrosis tubular aguda, nefritis } \\
\text { intersticial aguda, glomerulonefritis etc. }\end{array}$ \\
\hline Post-renal & $>4 \%$ & $\begin{array}{l}\text { Obstrucción (hiperplasia prostática } \\
\text { benigna, cálculos en la vejiga, obstrucción } \\
\text { ureteral bilateral). }\end{array}$ \\
\hline
\end{tabular}




\section{Bibliografía}

\section{Original/Primaria}

1. Espinel CH. La prueba FENa. Uso en el diagnóstico diferencial de la insuficiencia renal aguda. JAMA [Internet]. 1976 [citado marzo 2019];236(6):579-81. Disponible en: https: / / www.ncbi.nlm.nih.gov/ pubmed / 947239 .

\section{Validación}

1. Miller TR, Henrich WH, Schrier RW. Índices diagnósticos urinarios en insuficiencia renal aguda. Ann Intern Med [Internet]. 1978 [citado marzo 2019];89:47-50. Disponible en: https: / / www.ncbi.nlm.nih.gov / pubmed / 666184 .

2. Steiner RW. Interpretando la excreción fraccional de sodio. Am J Med [Internet] 1984 [citado marzo 2019]; 77.(4): 699-702. Disponible en: https: / / www.ncbi.nlm.nih.gov/ pubmed / 6486145. 


\section{Utilidad}

Determina la causa de la insuficiencia renal aguda. Es similar a la FENa, pero esta se puede usar en pacientes que usan diuréticos.

\section{Descripción}

- FEUrea se puede usar en pacientes que usan diuréticos, ya que la excreción de urea no se ve afectada significativamente por los diuréticos.

\section{Parámetros}

BUN

Creatinina en suero:

Urea en orina:

Creatinina en orina:
$8-20 \mathrm{mg} / \mathrm{dl}$

$0,7-1,3 \mathrm{mg} / \mathrm{dl}$

$350-700 \mathrm{mg} / \mathrm{dl}$

20-370 mg/dl

Fórmula

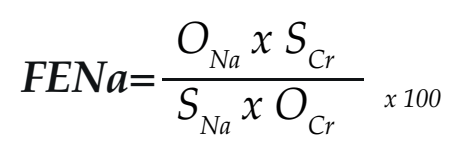

Interpretación

\begin{tabular}{|lll|}
\hline Insuficiencia renal & FENa & CAUSAS \\
\hline Pre-renal & $\leq 35 \%$ & $\begin{array}{l}\text { Hipovolemia, insuficiencia cardíaca, } \\
\text { estenosis de la arteria renal, Sepsis, } \\
\text { etc. }\end{array}$ \\
\hline Renal & $>50 \%$ & $\begin{array}{l}\text { Necrosis tubular aguda, nefritis } \\
\text { intersticial aguda, glomerulonefritis } \\
\text { etc. }\end{array}$ \\
\hline Post-renal & N/A & $\begin{array}{l}\text { Obstrucción (hiperplasia prostática } \\
\text { benigna, cálculos en la vejiga, } \\
\text { obstrucción ureteral bilateral). }\end{array}$ \\
\hline
\end{tabular}




\section{Bibliografía}

\section{Original/Primaria}

1. Kaplan AA, Kohn OF. Excreción fraccional de la urea como guía para la disfunción renal. Am J Nephrol [Internet]. 1992 [citado marzo 2019]; 12 (1-2): 49-54. Disponible en: https: / / www.ncbi.nlm.nih.gov/ pubmed / 1415365 .

\section{Validación}

1. Carvounis CP, Nisar S, Guro-Razuman S. Significado de la excreción fraccional de urea en el diagnóstico diferencial de insuficiencia renal aguda. Kidney Int [Internet]. 2002 [citado marzo 2019]; 62 (6): 2223-2229. Disponible en: https: / /www.ncbi.nlm.nih.gov/pubmed/12427149.

2. Darmon M, Vincent F, Dellamonica J, Schortgen F, Gonzalez F, Das V, et.al. Rendimiento diagnóstico de la excreción fraccional de urea en la evaluación de pacientes críticamente enfermos con lesión renal aguda: un estudio de cohorte multicéntrico. Crit Care [Internet]. 2011[citado marzo 2019]; 15(4): R178. Disponible en: https: / / www.ncbi.nlm.nih. gov/pmc/articles/PMC3387621/. 


\section{ENFERMEDAD RENAL CRÓNICA}

ECUACIÓN DE CKD-EPI PARA TASA DE FILTRACIÓN GLOMERULAR

\section{Utilidad}

Es una estimación de la tasa de filtración glomerular basada en la creatinina sérica.

\section{Descripción}

- Usar en pacientes con enfermedad renal crónica (no aguda), para medir la función renal.

- La ecuación de la CKD-EPI se desempeña de manera superior a la ecuación de MDRD en pacientes con GFR estimada normal o ligeramente reducida.

\section{Parámetros}

Género

Edad

Creatinina $\quad \mathrm{S}_{\mathrm{Cr}} \quad 0.7-1.3 \mathrm{mg} / \mathrm{dl}$

\section{Fórmula}

CKDEPI Creatitina $=A \times(S c r / B)^{C} \times 0.993^{\text {edad }} \times(1.159$ si es negro $)$

\begin{tabular}{|c|c|c|c|}
\hline \multicolumn{2}{|l|}{ Mujer } & \multicolumn{2}{|l|}{ Masculino } \\
\hline $\begin{array}{l}\text { Creatinina en suero } \\
\leq 0.7\end{array}$ & $\begin{array}{l}A=144 \\
B=0.7 \\
C=-0.329\end{array}$ & $\begin{array}{l}\text { Creatinina en suero } \\
\leq 0.9\end{array}$ & $\begin{array}{l}A=141 \\
B=0.9 \\
C=-0.411\end{array}$ \\
\hline $\begin{array}{l}\text { Creatinina en suero } \\
>0.7\end{array}$ & $\begin{array}{l}\mathrm{A}=144 \\
\mathrm{~B}=0.7 \\
\mathrm{C}=-1.209\end{array}$ & $\begin{array}{l}\text { Creatinina en suero } \\
>0.9\end{array}$ & $\begin{array}{l}A=141 \\
B=0.9 \\
C=-1.209\end{array}$ \\
\hline
\end{tabular}




\section{Interpretación}

\begin{tabular}{|l|l|}
\hline ETAPA DE IRC & TFG $\left(\mathrm{ml} / \mathrm{min} / 1,73 \mathrm{~m}^{2}\right)$ \\
\hline I & $\geq 90$ \\
\hline II & $60-89$ \\
\hline III & $30-59$ \\
\hline IV & $15-29$ \\
\hline V & $<15$ \\
\hline
\end{tabular}

\section{Bibliografía}

\section{Original/Primaria}

1. Levey AS, Stevens LA, Schmid CH, et al. Una nueva ecuación para estimar la tasa de filtración glomerular. Ann Intern Med [Internet]. 2009 [citado marzo 2019]; 150 (9): 604-12. Disponible en: https:// www.ncbi.nlm.nih.gov/pubmed/19414839.

2. Inker LA, Schmid CH, Tighiouart H, Eckfeldt JH, Feldman HI, Greene $\mathrm{T}$, et al. Estimación de la tasa de filtración glomerular a partir de creatinina sérica y cistatina C. N Engl J Med [Internet]. 2012 [citado marzo 2019]. 367 (1): 20-9. Disponible en: https: / / www.ncbi.nlm.nih. gov/pubmed/22762315. 


\section{ECUACIÓN DE MDRD GFR}

\section{Utilidad}

Calcula la tasa de filtración glomerular en función de la creatinina y las características del paciente.

\section{Descripción}

- Usar únicamente en enfermedad renal crónica (ERC); no es exacto para la insuficiencia renal aguda.

\section{Parámetros}

Sexo

Raza negra

Edad

Creatinina en suero $\quad \mathrm{Cr} \quad 0.7-1.3 \mathrm{mg} / \mathrm{dl}$

\section{Fórmula}

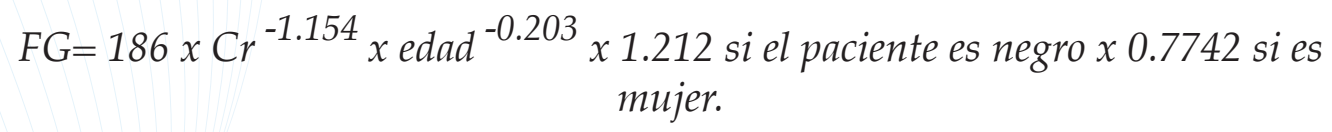

\section{Interpretación}

\begin{tabular}{|c|c|}
\hline ETAPA DE IRC & TFG $\left(\mathrm{ml} / \mathrm{min} / \mathbf{1 , 7 3} \mathrm{m}^{2}\right)$ \\
\hline I & $\geq 90$ \\
\hline II & $60-89$ \\
\hline III & $30-59$ \\
\hline IV & $15-29$ \\
\hline V & $<15$ \\
\hline
\end{tabular}

\section{Bibliografía}

\section{Original/Primaria}

1. Levey AS, Bosch JP, Lewis JB, Greene T, Rogers N, Roth D. Un método más preciso para estimar la tasa de filtración glomerular de la 
creatinina sérica: una nueva ecuación de predicción. Modificación de la dieta en el grupo de estudio de enfermedades renales. Ann Intern Med [Internet]. 1999 [citado marzo 2019]; 130 (6): 461-70. Disponible en: https: / / www.ncbi.nlm.nih.gov/ pubmed / 10075613.

\section{Validación}

1. Levey AS, Coresh J, Greene T, et al. Uso de valores de creatinina sérica estandarizados en la modificación de la dieta en la ecuación del estudio de la enfermedad renal para estimar la tasa de filtración glomerular. Ann Intern Med [Internet]. 2006 [citado marzo 2019]; 145 (4): 24754. Disponible en: https://annals.org/aim/article-abstract/727341/ using-standardized-serum-creatinine-values-modification-diet-renaldisease-study volume $=145 \&$ issue $=4 \&$ page $=247$.

2. Regla AD, Larson TS, Bergstralh EJ, Slezak JM, Jacobsen SJ, Cosio FG. Uso de creatinina sérica para estimar la tasa de filtración glomerular: precisión en la buena salud y en la enfermedad renal crónica. Ann Intern Med [Internet]. 2004 [citado marzo 2019]; 141:929_ 937. Disponible en: https: / / annals.org/aim/article-abstract/718024/ using-serum-creatinine-estimate-glomerular-filtration-rate-accuracygood-health ?volume $=141 \&$ issue $=12 \&$ page $=929 \# \mathrm{FN}$. 


\section{ACLARAMIENTO DE CREATININA-ECUACIÓN DE COCKCROFT- GAULT}

\section{Utilidad}

Se emplea para estimar el aclaramiento de creatinina.

\section{Descripción}

- El aclaramiento de creatinina todavía se utiliza como el estándar para la dosificación de fármacos, en lugar de GFR.

\section{Parámetros}

Sexo

Años

Peso

Creatinina

$\mathrm{kg}$

$0.7-1.3 \mathrm{mg} / \mathrm{dl}$

Fórmula

Cockcroft-Gault $=\frac{(140 \text {-edad }) \times(\text { peso en } \mathrm{kg}) \times(0.85 \text { si es mujer })}{72 \times \text { Creatinina }}$

\section{Interpretación}

\begin{tabular}{|c|c|}
\hline ETAPA DE IRC & TFG $\left(\mathrm{ml} / \mathrm{min} / \mathbf{1 , 7 3} \mathrm{m}^{2}\right)$ \\
\hline I & $\geq 90$ \\
\hline II & $60-89$ \\
\hline III & $30-59$ \\
\hline IV & $15-29$ \\
\hline V & $<15$ \\
\hline
\end{tabular}

\section{Bibliografía}

\section{Original/Primaria}

1. Cockcroft DW, Gault MH. Predicción del aclaramiento de creatinina a partir de creatinina sérica. Nephron [Internet]. 1976 [citado marzo 
2019];16(1):31-41. Disponible en: https://www.ncbi.nlm.nih.gov/ pubmed/1244564.

\section{Validación}

1. Invierno MA, Guhr KN, Berg GM. Impacto de diversos pesos corporales y concentraciones de creatinina sérica en el sesgo y la precisión de la ecuación de Cockcroft-Gault. Farmacoterapia [Internet]. 2012 [citado marzo 2019]; 32 (7): 604-12. Disponible en: https://www.ncbi.nlm. nih.gov/pubmed/22576791.

2. Brown DL, Masselink AJ, Lalla CD. Rango funcional del aclaramiento de creatinina para la dosificación renal de medicamentos: una solución práctica a la controversia de qué peso usar en la ecuación de Cockcroft-Gault. Ann Pharmacother [Internet]. 2013 [citado marzo 2019];47(7-8):1039-44. Dsiponible en: https: / / www.ncbi.nlm.nih.gov/ pubmed / 23757387. 


\section{CALCULADORA DE RIESGO DE INSUFICIENCIA RENAL}

\section{Utilidad}

Predice la progresión a insuficiencia renal en pacientes con enfermedad renal crónica (ERC).

\section{Descripción}

- Es probable que tenga una validez limitada en pacientes que están gravemente enfermos.

\section{Parámetros}

\begin{tabular}{|c|c|c|c|c|c|}
\hline CRITERIO & & PTS. & CRITERIO & & PTS \\
\hline \multirow{10}{*}{$\begin{array}{l}\mathrm{GFR}, \mathrm{mL} / \mathrm{min} \\
/ 1.73 \mathrm{~m}^{2}\end{array}$} & $10-14$ & -35 & \multirow{10}{*}{$\begin{array}{l}\text { Albúmina sérica } \\
\text { g/dl }\end{array}$} & $\geq 2,5$ & -5 \\
\hline & $15-19$ & -30 & & $2,6-3$ & 0 \\
\hline & $20-24$ & -25 & & $3,1-3,5$ & +2 \\
\hline & $25-29$ & -20 & & $\geq 3,6$ & +4 \\
\hline & $30-34$ & -15 & & & \\
\hline & $35-39$ & -10 & & & \\
\hline & $40-44$ & -5 & & & \\
\hline & $45-49$ & 0 & & & \\
\hline & $50-54$ & +5 & & & \\
\hline & $55-59$ & +10 & & & \\
\hline \multirow{4}{*}{ Sexo } & & & \multirow{4}{*}{$\begin{array}{l}\text { Fósforo en suero } \\
\mathrm{mg} / \mathrm{dl}\end{array}$} & $<3,5$ & +3 \\
\hline & Hombre & -2 & & $3,5-4,5$ & 0 \\
\hline & & 0 & & $4,6-5,5$ & -3 \\
\hline & & & & $>5,5$ & -5 \\
\hline
\end{tabular}




\begin{tabular}{|c|c|c|c|c|c|}
\hline $\begin{array}{l}\text { Proporción de } \\
\begin{array}{l}\text { albúmina } \\
\text { creatinia } \\
\text { orina }\end{array}\end{array}$ & $\begin{array}{l}<30 \\
3-300 \\
>300\end{array}$ & $\begin{array}{l}0 \\
-14 \\
-22\end{array}$ & $\begin{array}{l}\text { Bicarbonato En } \\
\text { suero } \mathrm{mEq} / \mathrm{L}\end{array}$ & $\begin{array}{c}<18 \\
18-22 \\
23-25 \\
>25\end{array}$ & $\begin{array}{l}-7 \\
-4 \\
-1 \\
0\end{array}$ \\
\hline Edad & $\begin{array}{l}<30 \\
30-39 \\
40-49 \\
50-59 \\
60-69 \\
70-79 \\
80-89 \\
\geq 90\end{array}$ & $\begin{array}{l}-4 \\
-2 \\
0 \\
+2 \\
+4 \\
+6 \\
+8 \\
+10\end{array}$ & $\begin{array}{l}\text { Calcio En suero } \\
\mathrm{mg} / \mathrm{dl}\end{array}$ & $\begin{array}{c}\leq 8.5 \\
8,6-9.5 \\
\geq 9,6\end{array}$ & $\begin{array}{l}-3 \\
0 \\
+2\end{array}$ \\
\hline
\end{tabular}

\section{Interpretación}

\section{PUNTUACIÓN DE RIESGO DE} INSUFICIENCIA RENAL
PROBABILIDAD DE INSUFICIENCIA RENAL A LOS 5 AÑOS

\begin{tabular}{c|c}
$<-41$ & $>90 \%$ \\
-41 & $89 \%$ \\
-40 & $86.9 \%$ \\
39 & $84.1 \%$ \\
38 & $81 \%$ \\
37 & $81 \%$ \\
36 & $74.4 \%$
\end{tabular}




\begin{tabular}{|c|c|}
\hline 35 & $70.9 \%$ \\
\hline 34 & $67.3 \%$ \\
\hline 33 & $63.6 \%$ \\
\hline 32 & $59.9 \%$ \\
\hline 31 & $56.3 \%$ \\
\hline 30 & $52.8 \%$ \\
\hline 29 & $49.3 \%$ \\
\hline 28 & $45.9 \%$ \\
\hline 27 & $42.7 \%$ \\
\hline 26 & $39.6 \%$ \\
\hline 25 & $36.6 \%$ \\
\hline 24 & $33.8 \%$ \\
\hline 23 & $31.2 \%$ \\
\hline 22 & $28.7 \%$ \\
\hline 21 & $26.4 \%$ \\
\hline 20 & $24.2 \%$ \\
\hline 19 & $22.2 \%$ \\
\hline 18 & $20.3 \%$ \\
\hline 17 & $18.6 \%$ \\
\hline 16 & $17 \%$ \\
\hline 15 & $15.5 \%$ \\
\hline 14 & $14.1 \%$ \\
\hline 13 & $12.9 \%$ \\
\hline 12 & $11.7 \%$ \\
\hline 11 & $10.7 \%$ \\
\hline
\end{tabular}




\begin{tabular}{|c|c|}
\hline $\mathbf{1 0}$ & $9.7 \%$ \\
$\mathbf{9}$ & $8.8 \%$ \\
7 & $8 \%$ \\
6 & $7.3 \%$ \\
5 & $6.6 \%$ \\
4 & $6 \%$ \\
$\geq-3$ & $5,5 \%$ \\
& $<5 \%$ \\
\hline
\end{tabular}

\section{Bibliografía}

\section{Original/Primaria}

1. Tangri N, Stevens LA, Griffith J, et al. Un modelo predictivo para la progresión de la enfermedad renal crónica a la insuficiencia renal. JAMA [Internet]. 2011 [citado marzo 2019]; 305(15):1553-9. Disponible en: https: / / jamanetwork.com/journals/ jama/fullarticle/ 897102.

\section{Validación}

1. Tangri N, Grams ME, Levey AS, et al. Evaluación multinacional de la precisión de las ecuaciones para predecir el riesgo de insuficiencia renal: un metanálisis. JAMA [Internet]. 2016 [citado marzo 2019]; 315 (2): 164-74. Disponible en: https://www.ncbi.nlm.nih.gov/ pubmed / 26757465. 


\section{FALLA RENAL AGUDA}

\section{CLASIFICACIÓN AKIN PARA LESIONES RENALES AGUDAS}

\section{Utilidad}

Clasifica la gravedad de la lesión renal aguda.

\section{Descripción}

- Los pacientes en terapia de reemplazo renal (TRR) se consideran en la etapa 3 independientemente de si cumplen con los criterios formales.

\section{Parámetros}

Para ser diagnosticado con lesión renal aguda según la definición de AKIN, el paciente debe tener al menos uno de los siguientes en las últimas 48 horas:

\section{CRITERIOS}

Incremento de la creatinina en el suero $0.3 \mathrm{mg} / \mathrm{dl}<$ desarrollado en 48 horas o $>50 \%$ desarrollado en 7 días.

Aumento de la creatinina sérica $\geq 1.5$ por encima del nivel basal.

Oliguria (producción de orina $<0,5 \mathrm{ml} / \mathrm{kg}$ por hora) durante $>6$ horas.

Luego, seleccione el criterio apropiado que conduzca a la etapa más acertada posible:

\begin{tabular}{|l|l|l|}
\hline $\begin{array}{l}\text { CLASIFICACIÓN } \\
\text { AKIN }\end{array}$ & \multicolumn{1}{|c|}{ CREATININA EN SUERO } & \multicolumn{1}{|c|}{$\begin{array}{c}\text { PRODUCCIÓN DE } \\
\text { ORINA }\end{array}$} \\
\hline $\begin{array}{l}\text { No lesión renal } \\
\text { aguda }\end{array}$ & $\begin{array}{l}\text { Creatinina normal, o }<1.5 \mathrm{x} \\
\text { desde la línea de base. }\end{array}$ & $\begin{array}{l}\geq 0.5 \mathrm{ml} / \mathrm{kg} \quad \text { por } \\
\text { hora por }>6 \text { horas. }\end{array}$ \\
\hline Estadio I & $\begin{array}{l}\text { Aumento absoluto } \geq 0.3 \mathrm{mg} / \mathrm{dl} \\
\text { o incremento } \geq \text { a un } 150-200 \% \\
\text { del basal. }\end{array}$ & $\begin{array}{l}<0.5 \mathrm{ml} / \mathrm{kg} \text { por } \\
\text { hora durante }>6 \\
\text { horas. }\end{array}$ \\
\hline
\end{tabular}




\begin{tabular}{|l|l|l|}
\hline Estadio II & $\begin{array}{l}\text { Incremento de la creatinina } \\
\text { sérica superior a un 200-300\% } \\
\text { del valor basal. }\end{array}$ & $\begin{array}{l}<0.5 \mathrm{ml} / \mathrm{kg} \text { por } \\
\text { hora durante }>12 \\
\text { horas. }\end{array}$ \\
\hline Estadio III & $\begin{array}{l}\text { Incremento de la creatinina } \\
\text { superior a un } 300 \% \text { de la basal } \\
\text { o creatinina sérica } \geq 4.0 \mathrm{mg} / \mathrm{dL} \\
\text { después de un aumento agudo } \\
\text { superior a } \geq 0.5 \mathrm{mg} / \mathrm{dl} .\end{array}$ & $\begin{array}{l}<0,3 \mathrm{ml} / \mathrm{kg} \text { por } \\
\text { hora durante } \geq 24 \\
\text { horas, o anuria } \\
\text { durante } 12 \text { horas. }\end{array}$ \\
\hline
\end{tabular}

\section{Bibliografía}

\section{Original/Primaria}

1. Mehta RL, Kellum JA, Shah SV, et.al. Red de lesiones renales agudas: informe de una iniciativa para mejorar los resultados de la lesión renal aguda. Crit Care [Internet]. 2007 [citado marzo 2019]; 11 (2): R31. Disponibleen:https: / ccforum.biomedcentral.com/articles/10.1186/ cc5713.

\section{Validación}

1. Lopes JA, Jorge S. Las clasificaciones RIFLE y AKIN para la lesión renal aguda: una revisión crítica y completa. Clin Kidney J [Internet]. 2013 [citado marzo 2019];6(1):8-14. Disponible en: https: / / www.ncbi. nlm.nih.gov/pubmed/27818745.

2. Xiong J, Tang X, Hu Z, Nie L, Wang Y, et al. The RIFLE versus AKIN classification for incidence and mortality of acute kidney injury in critical ill patients: A meta-analysis Scientific Reports [Internet]. 2015 [citado marzo 2019]. 17917. Disponible en: https: / / www.nature.com/ articles/srep17917.

3. Van Biesen W, Vanholder R, Lameire N. Definición de insuficiencia renal aguda: RIFLE y más allá. CJASN [Internet]. 2006 [citado marzo 2019]:1(6)1314-1319. Disponible en: https://cjasn.asnjournals.org/ content/1/6/1314.full. 


\section{CRITERIOS RIFLE PARA LA LESIÓN RENAL AGUDA}

\section{Utilidad}

Clasifica la gravedad de la lesión renal aguda, similar a la clasificación de AKIN.

\section{Descripción}

Recuerde que la lesión renal aguda, puede estar presente incluso con creatinina sérica normal, si la GFR está disminuida, o si la creatinina está significativamente elevada por encima del nivel basal.

\section{Parámetros}

\begin{tabular}{|c|c|c|c|}
\hline CRITERIOS & $\begin{array}{l}\text { CREATININA EN } \\
\text { SUERO }\end{array}$ & GFR & GASTO DE ORINA \\
\hline $\begin{array}{l}\text { Risk } \\
\text { RIFLE-R }\end{array}$ & $\begin{array}{l}\text { Incremento de la } \\
\text { creatinina en el } \\
\text { suero }>50 \% \text {. }\end{array}$ & $\begin{array}{l}>25 \% \text { de la } \\
\text { disminución } \\
\text { por debajo de } \\
\text { línea base. }\end{array}$ & $\begin{array}{l}<0,5 \mathrm{ml} / \mathrm{Kg} / \mathrm{h} \\
\text { por }>6 \text { horas }\end{array}$ \\
\hline $\begin{array}{l}\text { Injury } \\
\text { RIFLE-I }\end{array}$ & $\begin{array}{l}\text { Incremento de la } \\
\text { creatinina en el } \\
\text { suero }>100 \% \text {. }\end{array}$ & $\begin{array}{l}>50 \% \text { de la } \\
\text { disminución } \\
\text { por debajo de } \\
\text { línea base. }\end{array}$ & $\begin{array}{l}<0,5 \mathrm{ml} / \mathrm{Kg} / \mathrm{h} \\
\text { por }>12 \text { horas }\end{array}$ \\
\hline $\begin{array}{l}\text { Failure } \\
\text { RIFLE-F }\end{array}$ & $\begin{array}{l}\text { Incremento de la } \\
\text { creatinina en el } \\
\text { suero }>200 \% \text {. }\end{array}$ & $\begin{array}{l}>75 \% \text { de la } \\
\text { disminución } \\
\text { por debajo de } \\
\text { línea base. }\end{array}$ & $\begin{array}{l}<0,3 \mathrm{ml} / \mathrm{Kg} / \mathrm{h} \\
\text { por }>24 \mathrm{~h} \mathrm{o} \\
\text { anuria por } 12 \mathrm{~h} .\end{array}$ \\
\hline $\begin{array}{l}\text { Loss } \\
\text { RIFLE-L }\end{array}$ & \multicolumn{3}{|c|}{ Necesidad de terapia de reemplazo renal por $>4$ semanas. } \\
\hline $\begin{array}{l}\text { ESRD } \\
\text { RIFLE-E }\end{array}$ & \multicolumn{3}{|c|}{ Necesidad de terapia de reemplazo renal por $>3$ semanas. } \\
\hline
\end{tabular}




\section{Bibliografía}

\section{Original/Primaria}

1. Bellomo R, Ronco C, Kellum JA, Mehta RL, Palevsky P. Acute renal failure - definition, outcome measures, animal models, fluid therapy and information technology needs: the Second International Consensus Conference of the Acute Dialysis Quality Initiative (ADQI) Group. Crit Care [Internet]. 2004 [citado marzo 2019];8(4):R204-12. Disponible en: https: / / ccforum.biomedcentral.com/articles/10.1186/ cc2872.

\section{Validación}

1. Van Biesen W, Vanholder R, Lameire N. Defining acute renal failure: RIFLE and beyond. Clin J Am Soc Nephrol [Internet]. 2006 [citado marzo 2019];1(6):1314-9. Disponible en: https://cjasn.asnjournals. org/content/1/6/1314.full.

2. Lopes JA, Jorge S. Las clasificaciones RIFLE y AKIN para la lesión renal aguda: una revisión crítica y completa. CKJ [Internet]. 2012 [citado marzo 2019].6(1):8-14. Disponible en: https:/ / academic.oup. $\mathrm{com} / \mathrm{ckj} /$ article / 6/1/8/468698 Clin Kidney J [Internet]. 2013 [citado marzo 2019];6(1):8-14. Disponible en: https://www.ncbi.nlm.nih. gov/pubmed/27818745.

3. Xiong J, Tang X, Hu Z, Nie L, Wang Y, et al. The RIFLE versus AKIN classification for incidence and mortality of acute kidney injury in critical ill patients: A meta-analysis Scientific Reports [Internet]. 2015 [citado marzo 2019]. 17917. Disponible en: https: / / www.nature.com/ articles/srep17917. 


\section{HIPONATREMIA E HIPERNATREMIA}

CORRECCIÓN DE SODIO POR HIPONATREMIA E HIPERGLUCEMIA

\section{Utilidad}

Calcula el nivel de sodio real en pacientes con hiperglucemia.

\section{Parámetros}

Sodio

Glucosa

$$
\begin{aligned}
& 135-145 \mathrm{mEq} / \mathrm{L} \\
& 70-99 \mathrm{mg} / \mathrm{dl}
\end{aligned}
$$

Fórmula

$$
\mathrm{Na} \text { corregio }=\mathrm{Na}+\left(\frac{G \text { lucosa- } 100}{42}\right)
$$

\section{Bibliografía}

\section{Original/Primaria}

1. Katz MA. Hiponatremia inducida por hiperglucemia: cálculo de la depresión sérica sérica esperada. N Engl J Med [Internet]. 197318 de octubre [citado marzo 2019]; 289 (16): 843-4. Disponible en: https:/ / www.ncbi.nlm.nih.gov/pubmed/4763428.

\section{Validación}

1. Hillier TA, Abbott RD, Barrett EJ. Hiponatremia: evaluación del factor de corrección para la hiperglucemia. Am J Med [Internet]. Abril de 1999 [citado marzo 2019]; 106 (4): 399-403. Disponible en: https:// www.ncbi.nlm.nih.gov/pubmed/10225241. 


\section{DÉFICIT DE SODIO EN HIPONATREMIA}

\section{Utilidad}

Calcula la cantidad total de sodio que debe reemplazarse en pacientes con hiponatremia.

\section{Características}

¡Recuerde no corregir con demasiada rapidez, para evitar la encefalopatía pontina desmielinizante!

\section{Parámetros}

Sodio en suero $\quad \mathrm{Na} \quad 135-145 \mathrm{mEq} / \mathrm{L}$

Agua corporal total ACT

Coeficiente

Coef 0.6 hombres jóvenes

0.5 mujeres jóvenes y hombres ancianos

0.45 mujeres ancianas

0.4 obesos

Tasa de corrección:

Aguda: hasta $0.5 \mathrm{meq} / \mathrm{h}$ o hasta $10-12 \mathrm{meq} / 24 \mathrm{~h}$ o hasta $18 \mathrm{meq} / 48 \mathrm{~h}$.

Crónica: hasta $8 \mathrm{meq} / 24 \mathrm{~h}$.

Fórmula

$$
\begin{gathered}
A C T=\text { Peso en } \mathrm{kg} x \text { coef } \\
\text { Fórmula de Adrogue-Madias }=\frac{\text { Na infusión-Na paciente }}{A C T+1}
\end{gathered}
$$

\section{Bibliografía}

\section{Original/Primaria}

1. Adrogué HJ, Madias NE. Hiponatremia. N Engl J Med [Internet]. 2000 [citado 27 marzo 2019]; 342: 1581. Disponible en: https: / www.ncbi. nlm.nih.gov/pubmed/10824078.

\section{Validación}

1. OhMS, Uribarri J, Barrido D, et al. Peligro de mielinolisis pontina central en la deshidratación hipotónica y recomendación de tratamiento. Am 
J Med Sci [Internet]. 1989 [citado 27 marzo 2019]; 298: 4. Disponible en: https: / / www.ncbi.nlm.nih.gov/ pubmed/2750773.

2. Mohmand HK, Issa D, Ahmad Z, Cappuccio JD, Kouides RW, Sterns RH. Solución salina hipertónica para la hiponatremia: riesgo de sobrecorrección inadvertida. Clin J Am Soc Nephrol [Internet]. 2007 [citado marzo 2019]. 2 (6): 1110-7. Disponible en: https:/ / www.ncbi. nlm.nih.gov/pubmed/17913972.

3. Watson PE, Watson ID, Batt RD. Volúmenes de agua corporal total para machos y hembras adultos estimados a partir de simples mediciones antropométricas. Am J Clin Nutr [Internet]. 1980 [citado marzo 2019];33(1):27-39. Disponible en: https://www.ncbi.nlm.nih. gov/pubmed / 6986753. 


\section{DÉFICIT DE AGUA PARA HIPERNATREMIA}

\section{Utilidad}

Calcula el déficit de agua libre por el agua corporal total estimada.

\section{Características}

- Recuerde no corregir con demasiada rapidez para evitar Edema cerebral

- Esta herramienta proporciona una estimación del déficit de agua libre en función del peso corporal del paciente; Esto puede ser incorrecto en pacientes con aumento o pérdida significativa de peso (especialmente de fuentes de líquidos).

\section{Parámetros}

Sodio paciente Na paciente

Sodio deseado Na deseado

Agua corporal total ACT

Coeficiente Coef $\quad 0.6$ hombres jóvenes

0.5 mujeres jóvenes y hombres ancianos

0.45 mujeres ancianas

0.4 obesos

Metas de descenso de sodio

Hasta

$0.5 \mathrm{mEq} / \mathrm{h}$

$10-12 \mathrm{mEq} / 24 \mathrm{~h}$

$18 \mathrm{mEq} / 48 \mathrm{~h}$

\section{Fórmula}

$$
\begin{aligned}
\text { Fórmula Déficit de agua } & =\frac{\text { Na paciente-Na deseado }}{\text { Na deseado }} \times A C T \\
A C T & =\text { Peso en } \mathrm{kg} x \text { coef }
\end{aligned}
$$




\section{Bibliografía}

\section{Original/Primaria}

4. Adrogué HJ, Madias NE. Hipernatremia. N Engl J Med [Internet]. 2000 [citado 27 marzo 2019]; ;342(20):1493-9. Disponible en: https: / / www.ncbi.nlm.nih.gov/pubmed/10816188

\section{Validación}

1. Adrogué HJ, Madias NE. Hiponatremia. N Engl J Med [Internet]. 2000 [citado 27 marzo 2019]; 342: 1581. Disponible en: https: / www.ncbi. nlm.nih.gov/pubmed/10824078

2. Watson PE, Watson ID, Batt RD. Volúmenes de agua corporal total para machos y hembras adultos estimados a partir de simples mediciones antropométricas. Am J Clin Nutr [Internet]. 1980 [citado marzo 2019];33(1):27-39. Disponible en: https:/ / www.ncbi.nlm.nih. gov/pubmed / 6986753

3. Barsoum NR, Levine BS. Recetas actuales para la corrección de hiponatremia e hipernatremia: ¿son demasiado simples?. Neprhro. Dial. Transplant [Internet]. 2000 [citado 27 marzo 2019]; Disponible en: https: / / doi.org/10.1093/ndt/17.7.1176 


\section{HIPOPOTASEMIA E HIPERPOTASEMIA}

\section{GRADIENTE DE POTASIO TRANSTUBULAR (GKTT)}

\section{Utilidad}

Puede ayudar en la evaluación de la hiperpotasemia o hipopotasemia.

\section{Parámetros}

Potasio en orina

$$
25-100 \mathrm{mEq} / \mathrm{L} / \text { día }
$$

Potasio en suero

$3,5-5 \mathrm{mEq} / \mathrm{L}$

Osmolaridad de la orina

$500-800 \mathrm{mOsm} / \mathrm{kg}$

Osmolaridad sérica

$275-295 \mathrm{mOsm} / \mathrm{kg}$

\section{Fórmula}

$$
G T T K=\frac{\text { Potasio en orina } x \text { Osmolaridad sérica }}{\text { Osmolaridad urinaria } x \text { Potasio sérico }}
$$

\section{Interpretación}

- Los valores de GTTK entre 8 y 9 se consideran normales en pacientes con una dieta normal.

- Durante la hiperpotasemia $\left(\mathrm{K}^{+}>5.0 \mathrm{mEq} / \mathrm{L}\right)$, el GTTK debe ser $\geq 7$; los valores más bajos sugieren hipoaldosteronismo.

- Durante la hipopotasemia $\left(\mathrm{K}^{+}<3.5 \mathrm{mEq} / \mathrm{L}\right)$, el GTTK debe ser $<3$ (pérdidas extrarrenales); los valores mayores $(\geq 7)$ sugieren pérdida renal de potasio.

- El GTTK esperado en la hiperpotasemia es $>10$, lo que sugiere una excreción renal normal de potasio.

\section{Bibliografía}

\section{Original/Primaria}

1. Ethier JH, Kamel KS, Magner PO, Lemann J, Halperin ML. La concentración de potasio transtubular en pacientes con hipopotasemia e hiperpotasemia. Am J Kidney Dis [Internet]. 1990 [citado marzo 
2019]; 15 (4): 309-15. Disponible en: https:/ / www.ncbi.nlm.nih.gov/ pubmed / 2321642

\section{Validación}

1. Choi MJ, Ziyadeh FN. La utilidad del gradiente de potasio transtubular en la evaluación de la hiperpotasemia. J Am Soc Nephrol [Internet]. 2008 [citado marzo 2019]; 19(3):424-6. Disponible en: https:/ / www. ncbi.nlm.nih.gov/pubmed/18216310. 


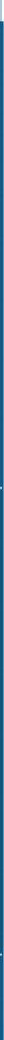

$$
\text { ENDOCRINOLOGÍA }
$$

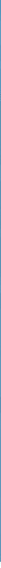




\section{DIABETES MELLITUS}

\section{SCORE FINDRISC}

\section{Utilidad}

Es un instrumento de cribaje que valora el riesgo individual de desarrollar diabetes mellitus tipo 2 en un plazo de 10 años.

\section{Parámetros}

\begin{tabular}{|l|c|c|}
\hline CRITERIO & CARACTERÍSTICAS & PUNTAJE \\
\hline Edad & $<45$ & 0 \\
& $45-54$ & 2 \\
& $55-64$ & 3 \\
\hline IMC & $>64$ & 4 \\
\hline Uso de medicamentos \\
para la presión arterial. & $25-30$ & 0 \\
\hline Antecedente de glucosa & $>30$ & 3 \\
\hline alta en la sangre. & No & 0 \\
\hline & Si & 2 \\
\hline Actividad física & Sí & 0 \\
\hline
\end{tabular}




\begin{tabular}{|l|c|c|}
\hline $\begin{array}{l}\text { Consumo de frutas y } \\
\text { verduras. }\end{array}$ & No & 1 \\
\hline Sí & No \\
\hline $\begin{array}{l}\text { Antecedentes familiares } \\
\text { de diabetes. }\end{array}$ & Sí, abuelos, tíos o primos \\
hermanos. & 0 \\
Sí, padres, hermanos o hijos. & 5 \\
\hline
\end{tabular}

IMC: indice de masa corporal.

\section{Interpretación}

\begin{tabular}{|c|l|c|c|}
\hline \multirow{2}{*}{ PUNTAJE } & \multirow{2}{*}{ RIESGO } & \multicolumn{2}{|c|}{$\begin{array}{c}\text { RIESGO DE DESARROLLAR } \\
\text { DMT2 EN 10 AÑ OS }\end{array}$} \\
\cline { 3 - 4 } & & Hombres & Mujeres \\
\hline $\mathbf{0 - 3}$ & Muy bajo & $0,3 \%$ & $0.1 \%$ \\
\hline $\mathbf{4 - 8}$ & Bajo & $0.8 \%$ & $0.4 \%$ \\
\hline $\mathbf{9 - 1 2}$ & Moderado & $2.6 \%$ & $2.2 \%$ \\
\hline $\mathbf{1 3 - 2 0}$ & Alto & $23.1 \%$ & $14.1 \%$ \\
\hline $\mathbf{2 1 1}$ & Muy alto & $50 \%$ & $50 \%$ \\
\hline
\end{tabular}

DMT2: Diabetes Mellitus tipo 2.

\section{Bibliografía}

\section{Original /Primaria}

1. Lindström J, Tuomilehto J. La puntuación de riesgo de diabetes: una herramienta práctica para predecir el riesgo de diabetes tipo 2. Diabetes Care [Internet]. 2003 [citado marzo 2019]; 26 (3): 725-31. Disponible en: https: / / www.ncbi.nlm.nih.gov/pubmed/12610029.

\section{Validación}

1. Makrilakis K, Liatis S, Grammatikou $S$, et al. Validación del 
cuestionario finlandés de riesgo de diabetes (FINDRISC) para la detección de diabetes tipo 2 no diagnosticada, disglucemia y el síndrome metabólico en Grecia.Diabetes Metab [Internet]. 2011 Abril [citado marzo 2019]; 37 (2): 144-51. Disponible en: https: / / www.ncbi. nlm.nih.gov/pubmed/21144787.

2. Zhang L,ZhanG Z, Zhang Y, Hu G, Chen L. Evaluación del puntaje de riesgo de diabetes finlandés en la detección de diabetes y prediabetes no diagnosticados entre adultos de EE. UU. Por género y raza: NHANES 1999-2010. Plos one[Internet]. mayo 2014 [citado marzo 2019]. Disponible en: https://journals.plos.org/plosone/ article?id=10.1371 / journal.pone.0097865.

3. Lindström J, Absetz P, Hemiö K, Peltomäki P, Peltonen M. Reducir el riesgo de diabetes tipo 2 con nutrición y actividad física: eficacia e implementación de intervenciones de estilo de vida en Finlandia. Public Health Nutr [Internet]. 2010 [citado marzo 2019];13(6A):993-9. Disponible en: https: / / www.ncbi.nlm.nih.gov/pubmed/20513271 . 


\section{PUNTAJE DE RIESGO DE HIPOGLUCEMIA}

\section{Utilidad}

Predice el riesgo de episodios de hipoglucemia de 12 meses en pacientes con Diabetes Mellitus tipo 2.

\section{Descripción}

- Ayuda a identificar a los pacientes de mayor riesgo, que podrían beneficiarse de las intervenciones dirigidas (por ejemplo, regímenes de medicamentos simplificados, monitoreo continuo de la glucosa, tratamiento de factores psicosociales y socioeconómicos).

\section{Parámetros}

\begin{tabular}{|l|c|}
\hline CRITERIO & PUNTAJE \\
\hline Admisiones de hipoglucemia. & $1-2$ \\
& $\geq 3$ \\
\hline $\begin{array}{l}\text { Visitas al servicio de emergencia en el último año por } \\
\text { alguna razón. }\end{array}$ & $<2$ \\
\hline Uso de insulina. & No \\
\hline Uso de sulfonilurea. & Sí \\
\hline Nonfermedad renal grave o terminal. & Sí \\
\hline & No \\
\hline Edad. & Sí \\
\hline
\end{tabular}




\section{Interpretación}

\begin{tabular}{|c|c|c|}
\hline CRITERIO & RIESGO & $\begin{array}{l}\text { RIESGO DE INGRESO } \\
\text { POR HIPOGLICEMIA } \\
\text { EN LOS PRÓXIMOS } 12 \\
\text { MESES }\end{array}$ \\
\hline $\begin{array}{l}\geq 3 \text { admisiones previas } \\
\text { por hipoglucemia o } 1-2 \\
\text { admisionesporhipoglucemia } \\
\text { + uso de insulina. }\end{array}$ & Alto & $>5 \%$ \\
\hline $\begin{array}{l}0 \text { admisiones previas por } \\
\text { hipoglucemia }+ \text { no uso } \\
\text { de insulina }+ \text { no uso de } \\
\text { sulfonilurea. }\end{array}$ & \multirow{3}{*}{ Bajo } & \multirow{3}{*}{$<1 \%$} \\
\hline $\begin{array}{l}0 \quad \text { admisiones previas } \\
\text { por hipoglucemia }+ \text { no } \\
\text { uso de insulina }+ \text { uso de } \\
\text { sulfonilurea }+ \text { edad }<77 \text { años } \\
\text { + no enfermedad renal grave } \\
\text { o en etapa terminal. }\end{array}$ & & \\
\hline $\begin{array}{l}0 \text { admisiones previas por } \\
\text { hipoglucemia }+ \text { uso de } \\
\text { insulina }+ \text { edad }<77 \text { años } \\
+<2 \text { visitas al servicio de } \\
\text { emergencias por alguna } \\
\text { razón. }\end{array}$ & & \\
\hline $\begin{array}{l}\text { Todas la demás } \\
\text { combinaciones de factores de } \\
\text { riesgo. }\end{array}$ & Intermedio & $1-5 \%$ \\
\hline
\end{tabular}

\section{Bibliografía}

\section{Original/Primaria}

1. Karter AJ, Warton EM, Lipska KJ, et al. Desarrollo y validación de una herramienta para identificar a pacientes con diabetes tipo 2 en alto riesgo de uso de hospital o de urgencias relacionado con hipoglucemia. JAMA Intern Med [Internet]. 2017 octubre [citado marzo 2019]; 177 (10): 1461-1470. Disponible en: https: / / jamanetwork.com/journals / jamainternalmedicine/ article-abstract/ 2649265. 


\section{LDL CALCULADO - FÓRMULA DE FRIEDEWALD}

\section{Utilidad}

Calcula LDL según el colesterol total, HDL y los triglicéridos.

\section{Descripción}

- Recuerde, los triglicéridos solo deben calcularse durante el ayuno.

\section{Parámetros}

Colesterol total $\mathrm{mg} / \mathrm{dl}$ Colesterol HDL $\quad \mathrm{mg} / \mathrm{dl}$ Triglicéridos $\mathrm{mg} / \mathrm{dl}$

Fórmula

$$
\text { Colesterol } L D L=\text { Colesterol total }-H D L-\left(\frac{\text { Triglicéridos }}{5}\right)
$$

\section{Interpretación}

\begin{tabular}{|l|l|}
\hline NIVEL LDL $\mathrm{mg} / \mathrm{dl}$ & Interpretación \\
\hline$<\mathbf{1 0 0}$ & Óptimo \\
\hline $\mathbf{1 0 0 - 1 2 9}$ & Casi óptimo \\
\hline $\mathbf{1 3 0 - 1 5 9}$ & Límite alto \\
\hline $\mathbf{1 6 0 - 1 8 9}$ & Alto \\
\hline $\mathbf{> 1 9 0}$ & Muy alto \\
\hline
\end{tabular}

\section{Bibliografía}

\section{Original/Primaria}

1. NIH, Managing blood colesterol in adults. Systematic evidence review from colesterol expert panel, 2013 [citado marzo 2019]. Disponible en: https: / / www.nhlbi.nih.gov/sites/default/files/media/docs/ cholesterol-in-adults.pdf . 


\section{Validación}

1. Cordova CM, Schneider CR, Juttel ID, Cordova MM. Comparación de la medición directa de colesterol LDL con la estimación utilizando la fórmula de Friedewald en una muestra de 10,664 pacientes. Arq Bras Cardiol [Internet]. 2004 [citado marzo 2019]; 83 (6): 482-7. Disponible en: https: / / www.ncbi.nlm.nih.gov/ pubmed / 15654445. 


\section{ESCALA DE WAGNER PARA PIE DIABÉTICO}

\section{Utilidad}

Estratifica las lesiones de pie diabético por las características de las úlceras.

\section{Descripción}

- Fácil de recordar, incorpora la profundidad de la úlcera, introduce las necrosis como lesiones no ulcerosas y se utiliza el grado 0 para definir al pie de riesgo.

- No hace referencia a la etiopatogenia de la lesión.

- La clasificación de Wagner, tampoco permite distinguir si una úlcera superficial presenta infección o isquemia.

\section{Parámetros}

\begin{tabular}{|c|c|c|}
\hline GRADO & PUNTAJE & CARACTERÍSTICA \\
\hline 0 & $\begin{array}{l}\text { Ninguna, pie de } \\
\text { riesgo }\end{array}$ & $\begin{array}{l}\text { Hiperqueratosis, cabezas de } \\
\text { metatarsianos prominentes, dedos en } \\
\text { garra y deformidades óseas. }\end{array}$ \\
\hline 1 & $\begin{array}{l}\text { Úlceras } \\
\text { superficiales }\end{array}$ & $\begin{array}{l}\text { Destrucción total del espesor de la } \\
\text { piel. }\end{array}$ \\
\hline 2 & Úlceras profundas & $\begin{array}{l}\text { Penetra en piel, grasa y ligamentos, } \\
\text { pero sin afectar hueso. Infectada. }\end{array}$ \\
\hline 3 & $\begin{array}{l}\text { Úlceras profundas } \\
+ \text { absceso }\end{array}$ & $\begin{array}{l}\text { Extensa y profunda, secreción y mal } \\
\text { olor. Existencia de osteomielitis. }\end{array}$ \\
\hline 4 & Gangrena limitada & $\begin{array}{l}\text { Necrosis de parte del pie (dedos, talón, } \\
\text { planta). }\end{array}$ \\
\hline 5 & Gangrena extensa & Todo el pie afectado, efectos sistémicos. \\
\hline
\end{tabular}




\section{Bibliografía}

\section{Original}

1. Roldán-Valenzuela, A. Cuidados en el pie diabético. Procedimiento y protocolos de Enfermería Hospital Universitario Virgen del Rocío. $1^{a}$ Ed. Sevilla. 2001.

\section{Validación}

1. González H, Mosquera A, Quintana M, Perdomo E, del Pino M. Clasificaciones de lesiones en pie diabético. Un problema no resuelto. Gerokomos [Internet]. 2012 [citado marzo 2019];23:2. 


\section{PUNTUACIÓN PEDIS PARA ÚLCERAS POR PIE DIABÉTICO}

\section{Utilidad}

Predice el riesgo de amputación y mortalidad a los 6 meses, en las úlceras del pie diabético.

\section{Descripción}

- Usar en pacientes hospitalizados con úlcera del pie diabético de cualquier duración.

- No usar en pacientes con diabetes secundaria o con úlceras en los pies causadas por enfermedades autoinmunes o enfermedades malignas.

\section{Parámetros}

\begin{tabular}{|l|l|c|}
\hline CRITERIO & CARACTERÍSTICAS & PUNTAJE \\
\hline \multirow{3}{*}{ Perfusión } & Sin enfermedad arterial periférica. & 0 \\
& $\begin{array}{l}\text { Enfermedad arterial periférica, sin isquemia } \\
\text { crítica de extremidades. }\end{array}$ & 1 \\
\hline \multirow{3}{*}{ Grado } & Isquemia crítica de extremidades. & 2 \\
\hline \multirow{5}{*}{ Profundidad intacta } & 0 \\
& $<1 \mathrm{~cm}^{2}$ & 1 \\
& $1-3 \mathrm{~cm}^{2}$ & 2 \\
& $>3 \mathrm{~cm}^{2}$ & 3 \\
\hline & Piel intacta & 0 \\
& Fascia, musculo, tendón & 2 \\
& Hueso o articulación & 3 \\
\hline
\end{tabular}




\begin{tabular}{|l|l|c|}
\hline \multirow{2}{*}{ Infección } & Ninguna & 0 \\
& Superficial & 1 \\
& Absceso, fascitis y o artritis séptica & 2 \\
& $\begin{array}{l}\text { Síndrome de respuesta inflamatoria } \\
\text { sistémica }\end{array}$ & 3 \\
\hline \multirow{2}{*}{ Sensación } & Sensación intacta & 0 \\
& Pérdida de sensibilidad & 1 \\
\hline
\end{tabular}

\section{Interpretación}

\begin{tabular}{|l|l|}
\hline PUNTAJE & RIESGO DE AMPUTACIÓN O MORTALIDAD \\
\hline$<7$ & Bajo \\
\hline$\geq 7$ & Alto \\
\hline
\end{tabular}

\section{Bibliografía}

\section{Original/Primaria}

1. Chuan F, Tang K, Jiang P, Zhou B, He X. Confiabilidad y validez del sistema de clasificación de perfusión, extensión, profundidad, infección y sensación (PEDIS) en pacientes con úlcera del pie diabético. Plos On [Internet]. 2015 [citado marzo 2019];10(4). Disponible en: https: / / journals.plos.org / plosone / article?id=10.1371 / journal.pone.0124739.

\section{Validación}

1. Uysal S, Arda B, Taşbakan MI, et al. Factores de riesgo para la amputación en pacientes con infección del pie diabético: un estudio prospectivo. Int Wound J [Internacional]. 2017 [citado marzo 2019]; 14(6):1219-1224. Disponible en: https://www.ncbi.nlm.nih.gov/ pubmed / 28722354 .

2. Ince $P, A b b a s ~ Z G$, Lutale JK, Basit A, Ali SM, Chohan F, et al. Uso del sistema de clasificación SINBAD y puntuación en la comparación del resultado del tratamiento de la úlcera del pie en tres continentes. Diabetes Care [Internet]. 2008 [citado marzo 2019] 31(5):964-7. Dsiponible en: https: / / www.ncbi.nlm.nih.gov/pubmed/18299441 . 


\section{CLASIFICACIÓN IDSA PARA SEVERIDAD DE INFECCIÓN DE PIE DIABÉTICO}

\section{Utilidad}

Clasifica las úlceras por pie diabético según la gravedad de la infección.

\section{Parámetros}

\begin{tabular}{|c|c|c|}
\hline $\begin{array}{l}\text { Severidad } \\
\text { criterio }\end{array}$ & CARACTERÍSTICAS & $\begin{array}{c}\text { Equivalente PUNTAJE } \\
\text { PEDIS } \\
\end{array}$ \\
\hline No infección & Herida sin pus ni inflamación. & Grado 1 \\
\hline Media & $\begin{array}{l}\text { No signos de infección sistémica. } \\
\text { Presencia de } 2 \text { signos de inflamación } \\
\text { (pus eritema dolor calor). } \\
\text { Celulitis no se extiende más allá de } \\
2 \mathrm{~cm} \text { de la úlcera. } \\
\text { Solo afecta a piel y TCS. }\end{array}$ & Grado 2 \\
\hline Moderada & $\begin{array}{l}\text { No signos de infección sistémica y } \\
\text { metabólicamente estable. } \\
\text { Celulitis se extiende más allá de } 2 \mathrm{~cm} \\
\text { de la úlcera. } \\
\text { Afecta músculos, tendones, } \\
\text { articulaciones o hueso. }\end{array}$ & Grado 3 \\
\hline Severa & $\begin{array}{l}\text { Infección en paciente con repercusión } \\
\text { sistémica. }\end{array}$ & Grado 4 \\
\hline
\end{tabular}

TCS: tejido celular subcutáneo.

\section{Bibliografía}

\section{Original/Primaria}

1. Berendt AR, Cornia PB, Pile JC, Peters EJ, Armstrong DG, Deery $\mathrm{HG}$, et al. Guía de práctica clínica de la Sociedad de Enfermedades Infecciosas de América del 2012 para el diagnóstico y tratamiento de las infecciones del pie diabético. Clin Infect Dis [Internet]. Junio 2012 [citado marzo 2019].;54 (12): e132-73. Disponible en: https: / www. ncbi.nlm.nih.gov/pubmed/22619242 


\section{CRISIS TIROTÓXICA}

\section{CRITERIOS DE BURCH Y WARTOFSKY}

\section{Utilidad}

Una crisis tirotóxica, se define como una exacerbación aguda de las manifestaciones del hipotiroidismo con disfunción orgánica múltiple, asociada a riesgo vital.

Esta escala diagnóstica permita diferenciar la tirotoxicosis no complicada, la crisis tiroidea inminente y la crisis establecida.

\section{Parámetros}

\begin{tabular}{|c|c|c|}
\hline CRITERIO & CARACTERÍSTICAS & PUNTAJE \\
\hline \multirow{6}{*}{ Temperatura } & $37.2-37.7$ & 5 \\
\hline & $37.8-38.2$ & 10 \\
\hline & $38.3-38.8$ & 15 \\
\hline & $38.9-39.3$ & 20 \\
\hline & $39.4-39.9$ & 25 \\
\hline & $>40$ & 30 \\
\hline \multirow{4}{*}{ Efectos del SNC } & Ausente & 0 \\
\hline & Leve (agitación) & 10 \\
\hline & Moderada (delirio-psicosis-letargo) & 20 \\
\hline & Severa (convulsiones, coma) & 30 \\
\hline \multirow{3}{*}{$\begin{array}{l}\text { Disfunción gastro- } \\
\text { intestinal }\end{array}$} & Ausente & 0 \\
\hline & $\begin{array}{l}\text { Moderara (diarrea, náuseas, vómitos, } \\
\text { dolor abdominal) }\end{array}$ & 10 \\
\hline & Severa (ictericia sin causa aparente) & 20 \\
\hline \multicolumn{3}{|c|}{ Disfunción cardiovascular } \\
\hline \multirow{5}{*}{ Taquicardia } & $90-109$ & 5 \\
\hline & $110-119$ & 10 \\
\hline & $120-129$ & 15 \\
\hline & 130-139 & 20 \\
\hline & $>140$ & 25 \\
\hline
\end{tabular}




\begin{tabular}{|l|c|c|}
\hline \multirow{2}{*}{$\begin{array}{l}\text { Insuficiencia } \\
\text { cardíaca congestiva }\end{array}$} & Ausente & 0 \\
& Leve (edema bimaleolar) & 5 \\
& Moderada (rales) & 10 \\
Fibrilación & Severa (edema pulmonar) & 15 \\
\hline auricular & Ausente & 0 \\
\hline \multirow{2}{*}{ Evento precipitante } & Presente & 10 \\
\hline
\end{tabular}

SNC: sistema nervioso central.

\section{Interpretación}

\section{PUNTAJE}

\begin{tabular}{|c|c|}
\hline $\mathbf{2 5}$ & $\begin{array}{c}\text { Tirotoxicosis no complicada, baja probabilidad de } \\
\text { crisis tiroidea. }\end{array}$ \\
\hline $\mathbf{2 5 - 4 4}$ & Crisis tiroidea inminente. \\
\hline $\mathbf{> 4 5}$ & Crisis tiroidea establecida. \\
\hline
\end{tabular}

\section{Bibliografía}

\section{Original/Primaria}

1. Burch HB, Wartofsky L. Tirotoxicosis potencialmente mortal. Tormenta tiroidea. Endocrinol Metab Clin North Am [Internet]. Junio 1993 [citado marzo 2019].22(2):263-77. Disponible en: https:/ / www.ncbi. nlm.nih.gov/ pubmed/ 8325286 .

\section{Validación}

1. Nayak B, Burman K. Tirotoxicosis y tormenta tiroidea. Endocrinol Metab Clin North Am [Internet]. Diciembre 2006 [citado marzo 2019]; 35 (4): 663-86, Disponible en: https: / / www.ncbi.nlm.nih.gov/ pubmed / 17127140

2. Batolomei S, Aranalde G, Keller L. Endcrinologia. Manual de Medicina Interna: Cálculos, scores y abordajes. 2a ed. Rosario-Argentina: Corpus Libros Médicos y Científicos; 2010. p. 422-423. 
CÁCULOSY

PRONÓSTICAS

EN MEDICINA INTERNA 


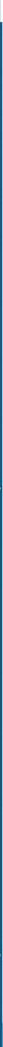

\section{REUMATOLOGÍA}

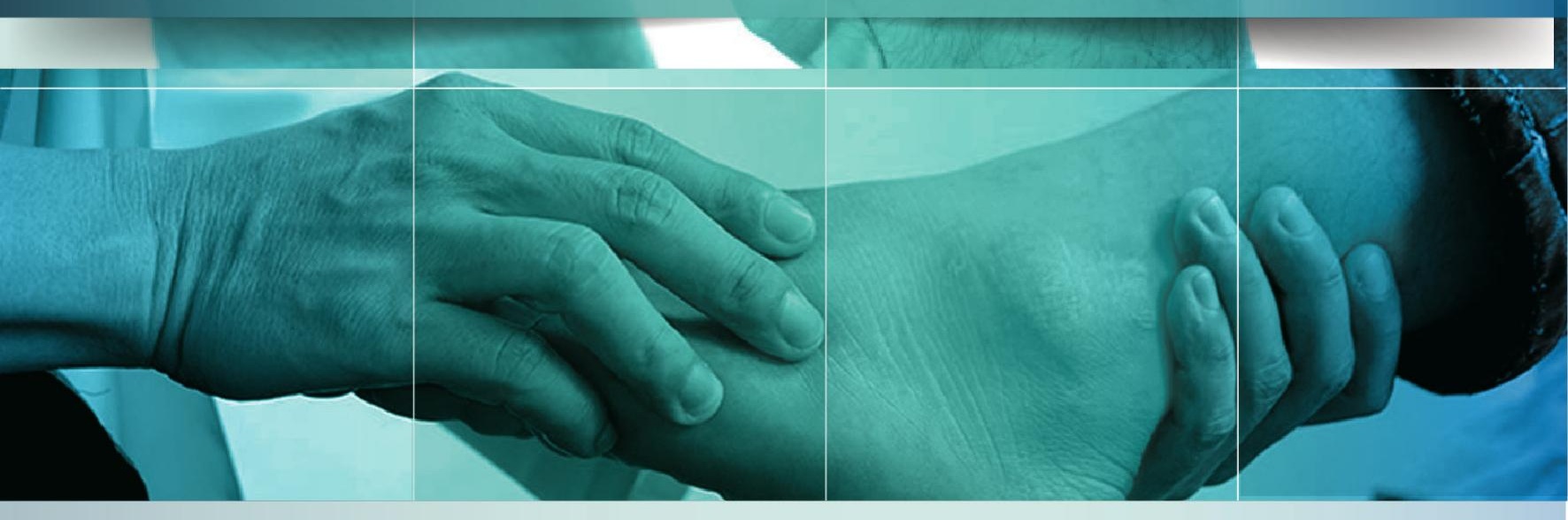




\section{ARTRITIS GOTOSA}

\section{CRITERIOS DE CLASIFICACIÓN DE GOTA ACR / EULAR}

\section{Utilidad}

Proporciona criterios diagnósticos formales para la gota.

\section{Descripción}

- Pacientes con mono u oligoartritis de etiología poco clara, donde la gota se encuentra en el diagnóstico diferencial.

- La clasificación ACR / EULAR se puede usar para diagnosticar formalmente la gota.

\section{Parámetros}

\begin{tabular}{|c|c|c|}
\hline CRITERIO & CARACTERÍSTICAS & PUNTAJE \\
\hline $\begin{array}{l}\text { Paso 1 - Criterio de } \\
\text { entrada } \\
\text { Si es afirmativo, se } \\
\text { requieren más de } 7 \\
\text { criterios de clasificación } \\
\text { para un diagnóstico } \\
\text { positivo. }\end{array}$ & $\begin{array}{l}\geq 1 \text { episodio de hinchazón, } \\
\text { dolor o sensibilidad en una } \\
\text { articulación / bursa periférica. }\end{array}$ & \\
\hline $\begin{array}{l}\text { Paso } 2-\text { Criterio } \\
\text { suficiente } \\
\text { En caso afirmativo, el } \\
\text { diagnóstico es positivo. }\end{array}$ & $\begin{array}{l}\text { Presencia de cristales de urato } \\
\text { monosódico (MSU) en una } \\
\text { articulación sintomática, bursa } \\
\text { o tifus. }\end{array}$ & \\
\hline
\end{tabular}




\begin{tabular}{|c|c|c|}
\hline $\begin{array}{l}\text { Patrón de afectación } \\
\text { articular/bursa durante } \\
\text { episodios. }\end{array}$ & $\begin{array}{l}\text { Articulación / bursa que no sea } \\
\text { tobillo, pie medio o primera } \\
\text { MTP o participación en una } \\
\text { poliartritis. } \\
\text { Tobillo o pie medio (como parte } \\
\text { del episodio monoarticular / } \\
\text { oligoarticular sin } 1^{\mathrm{a}} \text { MTP). } \\
\begin{array}{l}\text { ler MTP (como parte de un } \\
\text { episodio monoarticular u } \\
\text { oligoarticular). }\end{array}\end{array}$ & 2 \\
\hline $\begin{array}{l}\text { ¿Cuántas características } \\
\text { durante el episodio (s)? }\end{array}$ & No características & 0 \\
\hline \multirow{3}{*}{$\begin{array}{l}\text { Eritema sobre la } \\
\text { articulación (reportado } \\
\text { u observado); no puede } \\
\text { soportar el contacto o la } \\
\text { presión para unir; gran } \\
\text { dificultad para caminar } \\
\text { o incapacidad para usar } \\
\text { la articulación. }\end{array}$} & Una característica & 1 \\
\hline & Dos características & 2 \\
\hline & Tres características & 3 \\
\hline \multirow{3}{*}{$\begin{array}{l}\text { ¿Cuántos episodios con } \\
\text { los síntomas? }\end{array}$} & No hay episodios típicos & 0 \\
\hline & Un episodio típico & 1 \\
\hline & Episodios típicos recurrentes & 2 \\
\hline \multirow{2}{*}{ Evidencia de tofos } & Ausente & 0 \\
\hline & Presente & 4 \\
\hline \multirow{5}{*}{ Ácido úrico } & $<4 \mathrm{mg} / \mathrm{dL}$ & -4 \\
\hline & $\geq 4 \mathrm{o}<6 \mathrm{mg} / \mathrm{dL}$ & 0 \\
\hline & $\geq 6 \mathrm{o}<8 \mathrm{mg} / \mathrm{dL}$ & 2 \\
\hline & $\geq 8 \mathrm{o}<10 \mathrm{mg} / \mathrm{dL}$ & 3 \\
\hline & $\geq 10 \mathrm{mg} / \mathrm{dL}$ & 4 \\
\hline
\end{tabular}




\begin{tabular}{|l|l|c|}
\hline $\begin{array}{l}\text { Análisis del líquido } \\
\text { sinovial de unato } \\
\text { articulación o bursa } \\
\text { sintomática (siempre). }\end{array}$ & $\begin{array}{l}\text { Negativo ponosódico } \\
\text { No hecho }\end{array}$ & -2 \\
\hline $\begin{array}{l}\text { Evidencia por imágenes } \\
\text { de la deposición de } \\
\text { urato en la articulación } \\
\text { sintomática / bolsa. }\end{array}$ & Ausente o no hecho & 0 \\
\hline $\begin{array}{l}\text { Imágenes de evidencia } \\
\text { de daño articular } \\
\text { relacionado con la gota. }\end{array}$ & Ausente & 4 \\
$\begin{array}{l}\text { Radiografía de manos o } \\
\text { pies con } \geq 1 \text { erosión. }\end{array}$ & 0 \\
\hline
\end{tabular}

MTP: metatarsofalángica.

Interpretación

\section{DIAGNÓSTICO POSITIVO DE ARTRITIS GOTA}

Opción 1 criterio de entrada +7 puntos en criterios de clasificación

Opción 2 criterio suficiente

\section{Bibliografía}

\section{Original}

Neogi T, et al. Criterios de clasificación de gota 2015: una iniciativa de colaboración entre el Colegio Americano de Reumatología / Liga Europea contra el Reumatismo. Artritis \& amp; Reumatología [Internet]. 2015 [citado marzo 2019]; 67: 2557-2568. Disponible en: https: / / onlinelibrary. wiley.com/doi / full/10.1002/art.39254 .

\section{Validación}

Aggarwal R, Ringold S, Khanna D, Neogi T, Johnson SR, Miller A, et,al. ¿Distinciones entre los criterios de diagnóstico y clasificación?. Arthritis Care Res [Internet]. 2015 [citado marzo 2019]; 67: 891-897. Disponible en: https: / / www.ncbi.nlm.nih.gov/pubmed/25776731. 


\section{ARTRITIS REUMATOIDE}

CRITERIOS DE CLASIFICACIÓN DE LA ARTRITIS REUMATOIDE ACR / EULAR 2010

\section{Utilidad}

Es utilizada para el diagnóstico de artritis reumatoide (AR) en pacientes con sinovitis inflamatoria no diferenciada.

\section{Características}

- Se usa en pacientes que tienen al menos una articulación inflamada y no hay una mejor explicación para la sinovitis.

\section{Parámetros}

\begin{tabular}{|c|c|c|}
\hline CRITERIO & CARACTERÍSTICAS & PUNTAJE \\
\hline $\begin{array}{l}\text { Afectación } \\
\text { Cualquier articulación } \\
\text { inflamada / sensible en } \\
\text { el examen. }\end{array}$ & $\begin{array}{l}1 \text { articulación grande. } \\
\text { 2-10 articulaciones grandes. } \\
\text { 1-3 articulaciones pequeñas } \\
\text { (con o sin afectación de } \\
\text { articulaciones grandes). } \\
4-10 \text { articulaciones pequeñas } \\
\text { (con o sin afectación de } \\
\text { articulaciones grandes). } \\
>10 \text { articulaciones (al menos } 1 \\
\text { articulación pequeña). }\end{array}$ & $\begin{array}{l}0 \\
1 \\
2\end{array}$ \\
\hline $\begin{array}{l}\text { Serología } \\
\text { Se necesita al menos un } \\
\text { resultado de la prueba } \\
\text { de serología para usar } \\
\text { estos criterios. }\end{array}$ & $\begin{array}{l}\text { Factor reumatoide negativo } \\
\text { y anticuerpo proteico anti- } \\
\text { citrulinado negativo. } \\
\text { Factor reumatoide positivo } \\
\text { bajo o anticuerpo de proteína } \\
\text { anticitrulina poco positivo. } \\
\text { Factor reumatoide positivo } \\
\text { alto o anticuerpo de proteína } \\
\text { anticitrulina altamente positivo. }\end{array}$ & 3 \\
\hline
\end{tabular}




\begin{tabular}{|l|l|c|}
\hline $\begin{array}{l}\text { Reactivos de fase aguda } \\
\text { Necesita al menos un } \\
\text { resultado de prueba de } \\
\text { reactivo de fase aguda } \\
\text { para usar estos criterios. }\end{array}$ & PCR normal y VSG normal. & 0 \\
\hline $\begin{array}{l}\text { Duración de los } \\
\text { síntomas } \\
\begin{array}{l}\text { Por ejemplo, dolor, } \\
\text { hinchazón, sensibilidad. }\end{array}\end{array}$ & $\geq 6$ semanas. & 0 \\
\hline
\end{tabular}

\section{Interpretación}

\section{PUNTAJE}

\section{DIAGNÓSTICO}

\begin{tabular}{|l|l|}
\hline$\geq 6$ & Artritis reumatoide definida \\
\hline$<6$ & $\begin{array}{l}\text { No clasificable como teniendo RA por los criterios ACR / } \\
\text { EULAR }\end{array}$ \\
\hline
\end{tabular}

\section{Bibliografía}

\section{Original}

Aletaha D, Neogi T, Silman AJ, et al. 2010 Criterios de clasificación de la artritis reumatoide: una iniciativa colaborativa del Colegio Americano de Reumatología / Liga Europea contra el Reumatismo. Artritis reum [Internet]. 2010 [citado marzo 2019]; 62 (9): 2569-81. Disponible en: https: / / www.rheumatology.org / Portals / 0 / Files / 2010_revised_ criteria_classification_ra.pdf . 
ÍNDICE DE ACTIVIDAD DE LA ENFERMEDAD CLÍNICA (CDAI) PARA ARTRITIS REUMATOIDE

\section{Utilidad}

Determina la gravedad de la artritis reumatoide utilizando solo datos clínicos.

\section{Descripción}

- La evaluación de la actividad de la enfermedad en la artritis reumatoide (AR), debe considerarse como un proceso dinámico y continuo; por lo que se recomienda evaluar el CDAI en cada visita del paciente.

\begin{tabular}{|l|c|c|}
\hline CRITERIO & CARACTERÍSTICAS & PUNTAJE \\
\hline $\begin{array}{l}\text { Recuento de } \\
\text { articulaciones } \\
\text { dolorosas. }\end{array}$ & & $\begin{array}{c}\text { Número de } \\
\text { articulaciones } \\
0-28\end{array}$ \\
\hline $\begin{array}{l}\text { Recuento de } \\
\text { articulaciones } \\
\text { inflamadas. }\end{array}$ & &
\end{tabular}




\begin{tabular}{|l|l|l|}
\hline $\begin{array}{l}\text { Valora ción } \\
\text { global del } \\
\text { paciente de la } \\
\text { actividad de la } \\
\text { enfermedad. }\end{array}$ & $\begin{array}{l}\text { Teniendo en cuenta todas las formas } \\
\text { en una escala del 1 al 10, ¿cómo se } \\
\text { siente actualmente? }\end{array}$ & $1-10$ \\
\hline $\begin{array}{l}\text { Valor a ción } \\
\text { global del } \\
\text { médico de la } \\
\text { actividad de la } \\
\text { enfermedad. }\end{array}$ & $\begin{array}{l}\text { Según su opinión medica, } \\
\text { considerando todas las formas en las } \\
\text { que la artitis afecta al paciente, en una } \\
\text { escala del 1 al 10, ¿qué tan bien está? }\end{array}$ & $1-10$ \\
\hline
\end{tabular}

\section{Parámetros}

\section{Interpretación}

CDAI $=$ Recuento de articulaciones dolorosas + recuento de articulaciones inflamadas + valoración global del paciente + valoración global del médico.

\section{PUNTAJE GRAVEDAD DE LA ENFERMEDAD}

\begin{tabular}{|c|l|}
\hline$\leq 2.8$ & En remisión \\
\hline$>2.8-10$ & Bajo \\
\hline$>10-22$ & Moderado \\
\hline$>22$ & Alto \\
\hline
\end{tabular}

\section{Bibliografía}

\section{Original / Primaria}

1. Aletaha D, Nell VP, Stamm T, et al. Los reactantes de fase aguda agregan poco a los índices de actividad de la enfermedad compuesta para la artritis reumatoide: validación de un puntaje de actividad clínica. Arthritis Res Ther [Internet]. 2005 [citado marzo 2019]; 7(4): R796-R806. Disponible en: https://www.ncbi.nlm.nih.gov/pmc/ articles / PMC1175030 / . 


\section{Validación}

2. Singh $\mathrm{H}$, et al. Uso de la puntuación del índice de actividad de la enfermedad clínica para evaluar la actividad de la enfermedad en pacientes con artritis reumatoide: una experiencia india. Arthritis [Internet]. 2011 [citado marzo 2019],146398, 5. Disponible en: https:/ / www.hindawi.com/journals / arthritis/2011/146398/

3. Aletaha D, Smolen JS. El índice simplificado de actividad de la enfermedad (SDAI) y el índice de actividad de la enfermedad clínica (CDAI, por sus siglas en inglés) para monitorear a los pacientes en atención clínica estándar. Best Pract Res Clin Rheumatol [Internet]. Agosto 2007 [citado marzo 2019]; 21 (4): 663-75. Disponible en: https: / / www.ncbi.nlm.nih.gov/pubmed/17678828 . 


\section{DAS28-PCR (PUNTUACIÓN DE ACTIVIDAD DE LA ENFERMEDAD-28 PARA LA ARTRITIS REUMATOIDE CON PCR)}

\section{Utilidad}

Describe la gravedad de la artritis reumatoide, utilizando datos clínicos y de laboratorio, específicamente la PCR.

\section{Descripción}

- Las puntuaciones DAS, indican qué tan activa está actualmente la artritis reumatoide (AR) de un paciente, y puede ser mejorada con el tiempo.

\section{Parámetros}

\begin{tabular}{|l|c|c|c|}
\hline CRITERIO & CARACTERÍSTICAS & PUNTAJE \\
\hline $\begin{array}{l}\text { Recuento de } \\
\text { articulaciones } \\
\text { dolorosas. }\end{array}$ & $\begin{array}{l}\text { Número de } \\
\text { articulaciones }\end{array}$ & $0-28$ \\
\hline $\begin{array}{l}\text { Recuento de } \\
\text { articulaciones } \\
\text { inflamadas. }\end{array}$ & & \\
\hline $\begin{array}{l}\text { Proteína } \\
\text { reactiva. }\end{array}$
\end{tabular}




\begin{tabular}{|l|l|l|}
\hline Salud global. & $\begin{array}{l}\text { Teniendo en cuenta todas las formas } \\
\text { en que la artritis reumatoide le afecta, } \\
\text { en una escala del 1 al 10, ¿cómo se } \\
\text { siente actualmente? }\end{array}$ & $1-10$ \\
\hline
\end{tabular}

\section{Interpretación}

DAS28-PCR $=(0.56 \times \sqrt{ }$ (número articulaciones dolorosas $)+0.28 \times \sqrt{ }$ (número de articulaciones inflamadas) $+0.36 \times \ln (\mathrm{CRP}+1)+0.014 \times$ global del paciente +0.96$)$.

\section{PUNTAJE GRAVEDAD DE LA ENFERMEDAD}

\begin{tabular}{|l|l|}
\hline$<2.6$ & En remisión \\
\hline$\geq 2.6-3.2$ & Bajo \\
\hline$\geq 3.2-\leq 5.1$ & Moderado \\
\hline$>5.1$ & Alto \\
\hline
\end{tabular}

\section{Bibliografía}

\section{Original/Primaria}

1. Van der Heijde DM, Van 't hof MA, Van riel PL, et al. Evaluación de la actividad de la enfermedad en la práctica clínica de la artritis reumatoide: primer paso en el desarrollo de una puntuación de actividad de la enfermedad. Ann Rheum Dis [Internet]. 1990 [citado marzo 2019]; 49 (11): 916-20. Disponible en: https:/ / www.ncbi.nlm. nih.gov/pubmed/2256738.

\section{Validación}

1. Tamhane A, Redden DT, McGwin G, et al. Comparación del puntaje de actividad de la enfermedad mediante la tasa de sedimentación de eritrocitos y la proteína $C$ reactiva en los afroamericanos con artritis reumatoide. J Rheumatol. 2013 [citado marzo 2019]; 40 (11): 18121822. Disponible en: https://www.ncbi.nlm.nih.gov/pmc/articles/ PMC3987124/. 
2. Inoue E, Yamanaka H, Hara M, Tomatsu T, Kamatani N. Comparación de la tasa de sedimentación del eritrocito en la puntuación de actividad de la enfermedad (DAS) 28 y valores de umbral de proteína DAS28- C reactiva. Ann Rheum Dis [Internet]. Marzo 2007 [citado marzo 2019]; 66 (3): 407-409. Disponible en: https: / / www.ncbi.nlm.nih.gov/ pmc/ articles / PMC1856019/.

3. Hensor EMA, Emery P, Bingham S, Conaghan P. Discrepancias en la clasificación de pacientes con artritis reumatoide según DAS-28 (ESR) y DAS-28 (CRP): ¿se pueden reducir?. Rheumatology [Internet]. 2010 [citado marzo 2019):49 (8): 1521-1529. Disponible en: https:// academic.oup.com/rheumatology/article/49/8/1521/1790122 . 


\section{DAS28-VSG (PUNTUACIÓN DE ACTIVIDAD DE LA ENFERMEDAD-28 PARA LA ARTRITIS REUMATOIDE CON VSG)}

\section{Utilidad}

Describe la gravedad de la artritis reumatoide, utilizando datos clínicos y de laboratorio, específicamente la VSG.

\section{Descripción}

- Las puntuaciones DAS, indican qué tan activa está actualmente la artritis reumatoide (AR) de un paciente, y puede ser mejorada con el tiempo.

\section{Parámetros}

\begin{tabular}{|c|c|c|}
\hline CRITERIO & CARACTERÍSTICAS & PUNTAJE \\
\hline $\begin{array}{l}\text { Recuento de } \\
\text { articulaciones } \\
\text { dolorosas. }\end{array}$ & & $\begin{array}{c}\text { Número de } \\
\text { articulaciones } \\
0-28\end{array}$ \\
\hline $\begin{array}{l}\text { Recuento de } \\
\text { articulaciones } \\
\text { inflamadas. }\end{array}$ & & $\begin{array}{c}\text { Número de } \\
\text { articulaciones } \\
0-28\end{array}$ \\
\hline $\begin{array}{l}\text { Proteína C } \\
\text { reactiva. }\end{array}$ & $\mathrm{mg} / \mathrm{h}$ & \\
\hline
\end{tabular}




\begin{tabular}{|c|c|c|}
\hline Salud global. & $\begin{array}{c}\text { Teniendo en cuenta todas las formas } \\
\text { en que la artritis reumatoide le afecta, } \\
\text { en una escala del 1 al 10, ¿cómo se } \\
\text { siente actualmente? }\end{array}$
\end{tabular}$\quad 1-10$

\section{Interpretación}

DAS28-VSG $=(0.56 \times \sqrt{ }$ (número articulaciones dolorosas $)+0.28 \times \sqrt{ }$ (número de articulaciones inflamadas) +0.7 x VSG $+0.014 \times$ salud global del paciente.

\begin{tabular}{|c|c|}
\hline PUNTAJE & GRAVEDAD DE LA ENFERMEDAD \\
\hline$<2.6$ & En remisión \\
\hline$\geq 2.6-3.2$ & Bajo \\
\hline $\begin{array}{c}\geq 3.2-\leq \\
5.1\end{array}$ & Moderado \\
\hline$>5.1$ & Alto \\
\hline
\end{tabular}

\section{Bibliografía}

\section{Original/Primaria}

1. Van der Heijde DM, Van 't hof MA, Van riel PL, et al. Evaluación de la actividad de la enfermedad en la práctica clínica de la artritis reumatoide: primer paso en el desarrollo de una puntuación de actividad de la enfermedad. Ann Rheum Dis [Internet]. 1990 [citado marzo 2019]; 49 (11): 916-20. Disponible en: https:/ / www.ncbi.nlm. nih.gov/pubmed/2256738 .

\section{Validación}

1. Tamhane A, Redden DT, McGwin G, et al. Comparación del puntaje de actividad de la enfermedad mediante la tasa de sedimentación de eritrocitos y la proteína $C$ reactiva en los afroamericanos con artritis reumatoide. J Rheumatol. 2013 [citado marzo 2019]; 40 (11): 18121822. Disponible en: https: / /www.ncbi.nlm.nih.gov/pmc/articles / PMC3987124/ .

2. Inoue E, Yamanaka H, Hara M, Tomatsu T, Kamatani N. Comparación de la tasa de sedimentación del eritrocito en la puntuación de actividad 
de la enfermedad (DAS) 28 y valores de umbral de proteína DAS28-C reactiva. Ann Rheum Dis [Internet]. Marzo 2007 [citado marzo 2019]; 66 (3): 407-409. Disponible en: https:/ / www.ncbi.nlm.nih.gov/pmc/ articles/PMC1856019/.

3. Hensor EMA, Emery P, Bingham S, Conaghan P. Discrepancias en la clasificación de pacientes con artritis reumatoide según DAS-28 (ESR) y DAS-28 (CRP): ¿se pueden reducir?. Rheumatology [Internet]. 2010 [citado marzo 2019):49 (8): 1521-1529. Disponible en: https:/ / academic.oup.com/rheumatology/article/49/8/1521/1790122. 


\section{SDAI (ÍNDICE DE ACTIVIDAD DE LA ENFERMEDAD)}

\section{Utilidad}

Determina la gravedad de la artritis reumatoide, utilizando datos clínicos y de laboratorio.

\section{Parámetros}

\begin{tabular}{|c|c|c|}
\hline CRITERIO & CARACTERÍSTICAS & PUNTAJE \\
\hline $\begin{array}{l}\text { Recuento de } \\
\text { a r t i c u l a ci on e s } \\
\text { dolorosas. }\end{array}$ & & $\begin{array}{c}\text { Número de } \\
\text { articulaciones } \\
0-28\end{array}$ \\
\hline $\begin{array}{l}\text { Recuento de } \\
\text { a rt i c u l a ci o n e s } \\
\text { inflamadas. }\end{array}$ & & $\begin{array}{c}\text { Número de } \\
\text { articulaciones } \\
0-28\end{array}$ \\
\hline Proteína $\mathrm{C}$ reactiva. & $\mathrm{mg} / \mathrm{h}$ & \\
\hline $\begin{array}{l}\text { Valoración global } \\
\text { del paciente de } \\
\text { la actividad de la } \\
\text { enfermedad. }\end{array}$ & $\begin{array}{l}\text { Teniendo en cuenta todas } \\
\text { las formas en que la artritis } \\
\text { reumatoide le afecta, en una } \\
\text { escala del } 1 \text { al 10, ¿cómo se } \\
\text { siente actualmente? }\end{array}$ & $1-10$ \\
\hline
\end{tabular}




\begin{tabular}{|l|c|c|}
\hline $\begin{array}{l}\text { Valoración global } \\
\text { del médico de la } \\
\text { actividad de la } \\
\text { enfermedad. }\end{array}$ & $\begin{array}{c}\text { Según su opinión medica } \\
\text { considerando todas las formas } \\
\text { en las que la artritis afecta al } \\
\text { paciente, en una escala del 1 al } \\
10, \text { ¿qué tan bien está? }\end{array}$ & \multirow{1}{*}{$1-10$} \\
\hline
\end{tabular}

Interpretación

DAI = número de articulaciones dolorosas + número de articulaciones inflamadas + PCR + valoración global del paciente + valoración global del médico

\section{PUNTAJE GRAVEDAD DE LA ENFERMEDAD}

\begin{tabular}{|c|l|}
\hline$\geq 3.3$ & En remisión \\
\hline$\geq 3.3-11$ & Bajo \\
\hline$\geq 11-26$ & Moderado \\
\hline$>26$ & Alto \\
\hline
\end{tabular}

\section{Bibliografía}

\section{Original/Primaria}

1. Smolen JS, et al. Un índice de actividad de la enfermedad simplificado para la artritis reumatoide para uso en la práctica clínica Reumatology [Internet]. 2003 [citado marzo 2019]: 42 (2): 244-257.

\section{Validación}

1. Aletaha D, Smolen JS. El índice simplificado de actividad de la enfermedad (SDAI) y el índice de actividad de la enfermedad clínica (CDAI) para controlar a los pacientes en la atención clínica estándar. Best Pract Res Clin Rheumatol [Internet]. 2007 [citado marzo 2019];21(4):663-75. Disponible en: https://www.ncbi.nlm.nih.gov/ pubmed/17678828. 


\section{LUPUS ERITEMATOSOS SISTÉMICO}

\section{CRITERIOS PARA DIAGNÓSTICO DE LUPUS ERITEMATOSO SISTÉMICO}

\section{Utilidad}

Proporciona criterios diagnósticos para Lupus Eritematoso Sistémico.

Parámetros

\begin{tabular}{|c|c|}
\hline CRITERIO & DEFINICIÓN \\
\hline Eritema & $\begin{array}{l}\text { Eritema fijo, liso o elevado, sobre las eminencias } \\
\text { malares, con tendencia a respetar los surcos } \\
\text { nasolabiales. }\end{array}$ \\
\hline Lupus discoide & $\begin{array}{l}\text { Placas eritematosas elevadas con escamas } \\
\text { queratósicas adherentes y espículas foliculares; } \\
\text { en lesiones antiguas puede existir cicatrización } \\
\text { atrófica. }\end{array}$ \\
\hline Fotosensibilidad & $\begin{array}{l}\text { Exantema cutáneo como resultado de una anormal } \\
\text { reacción a la luz solar, según la anamnesis del } \\
\text { enfermo o por observación de un médico. }\end{array}$ \\
\hline Úlceras orales & $\begin{array}{l}\text { Úlceras orales o nasofaríngeas, en general } \\
\text { indoloras, observadas por un médico. }\end{array}$ \\
\hline Artritis & $\begin{array}{l}\text { Artritis no erosiva que afecta dos o más } \\
\text { articulaciones periféricas, caracterizada por dolor, } \\
\text { tumefacción o derrame. }\end{array}$ \\
\hline Serositis & $\begin{array}{l}\text { a) Pleuritis: historia compatible de dolor pleurítico } \\
\text { o roce auscultado por un médico o evidencia de } \\
\text { derrame pleural. } \\
\text { b) Pericarditis: confirmada por ECG o roce } \\
\text { pericárdico o evidencia de derrame pericárdico. }\end{array}$ \\
\hline Afección renal & $\begin{array}{l}\text { a) Proteinuria: persistente superior a } 500 \mathrm{mg} / 24 \\
\text { hs. O superior a } 3+\text { si no se cuantifica. } \\
\text { b) Cilindros celulares: integrados por hematíes o } \\
\text { hemoglobina, o de tipo granular, tubular o mixto. }\end{array}$ \\
\hline
\end{tabular}




\begin{tabular}{|l|l|}
\hline \multirow{2}{*}{$\begin{array}{l}\text { Afección } \\
\text { neurológica }\end{array}$} & $\begin{array}{l}\text { a) Convulsiones: en ausencia de fármacos } \\
\text { inductores de estas o alteraciones conocidas } \\
\text { del metabolismo (p. ej., uremia, cetoacidosis o } \\
\text { trastornos electrolíticos). } \\
\text { b) Psicosis: en ausencia de fármacos inductores de } \\
\text { ésta o alteraciones conocidas del metabolismo (p. } \\
\text { ej., uremia, cetoacidosis o trastornos electrolíticos). }\end{array}$ \\
\hline $\begin{array}{l}\text { Alteración } \\
\text { hematológica }\end{array}$ & $\begin{array}{l}\text { a) Anemia hemolítica: con reticulocitosis, } \\
\text { b) Leucopenia: inferior a 4 000/mL en dos o más } \\
\text { determinaciones. } \\
\text { c) Linfopenia: inferior a 1 500/mL en dos o más } \\
\text { determinaciones. } \\
\text { d) Trombocitopenia inferior a 100 000/mL en } \\
\text { ausencia de fármacos inductores. }\end{array}$ \\
\hline Anticuerpos \\
antinucleares
\end{tabular}




\section{Interpretación}

\section{DIAGNÓSTICO POSITIVO}

Si tiene 4 o más de estos criterios.

\section{Bibliografía}

\section{Original/Primaria}

1. Batolomei S, Aranalde G, Keller L. Reumatología. Manual de Medicina Interna: Cálculos, scores y abrodajes. 2a ed. Rosario-Argentina: Corpus Libros Médicos y Científicos; 2010. p. 422-423 . 


\section{ÍNDICE DE ACTIVIDAD DE LUPUS ERITEMATOSO SISTÉMICO - SLEDAI}

\section{Utilidad}

Es un instrumento que determina con certeza el grado de actividad o la intensidad del brote del Lupus Eritematoso Sitémico en un momento dado.

\begin{tabular}{|c|c|c|}
\hline CRITERIO & DEFINICIÓN & PUNTAJE \\
\hline Convulsiones & $\begin{array}{l}\text { De comienzo reciente. Excluir causas } \\
\text { infecciosas, metabólicas y fármacos. }\end{array}$ & 8 \\
\hline Psicosis & $\begin{array}{l}\text { Habilidad alterada para la función } \\
\text { diaria debido a alteración grave en } \\
\text { la percepción de la realidad. Incluye } \\
\text { alucinaciones, incoherencia, asociaciones } \\
\text { ilógicas, contenido mental escaso, } \\
\text { pensamiento ilógico, raro, desorganizado y } \\
\text { comportamiento catatónico. Excluir I. renal } \\
\text { y fármacos }\end{array}$ & 8 \\
\hline $\begin{array}{l}\text { Síndrome } \\
\text { orgánico } \\
\text { cerebral }\end{array}$ & $\begin{array}{l}\text { Función mental alterada con falta de } \\
\text { orientación, memoria, u otras funciones } \\
\text { intelectuales, de comienzo rápido y } \\
\text { manifestaciones clínicas fluctuantes. } \\
\text { Incluye disminución del nivel de conciencia } \\
\text { con capacidad reducida para focalizar, e } \\
\text { inhabilidad para mantener la atención en el } \\
\text { medio, más, al menos dos de los siguientes: } \\
\text { alteración de la percepción, lenguaje } \\
\text { incoherente, insomnio o mareo matutino, } \\
\text { o actividad psicomotora aumentada o } \\
\text { disminuida. Excluir causas infecciosas, } \\
\text { metabólicas y fármacos. }\end{array}$ & 8 \\
\hline $\begin{array}{l}\text { Alteraciones } \\
\text { visuales }\end{array}$ & $\begin{array}{l}\text { Retinopatía lúpica. Incluye cuerpos } \\
\text { citoides, hemorragias retinianas, exudados } \\
\text { serosos y hemorragias en la coroides, o } \\
\text { neuritis óptica. Excluir HTA, infección o } \\
\text { fármacos. }\end{array}$ & 8 \\
\hline $\begin{array}{l}\text { Alteraciones de } \\
\text { pares craneales }\end{array}$ & De reciente comienzo, motor o sensitivo & 8 \\
\hline
\end{tabular}




\begin{tabular}{|c|c|c|}
\hline Cefalea lúpica & $\begin{array}{l}\text { Grave, persistente; puede ser migrañosa } \\
\text { pero no responde a analgésicos narcóticos }\end{array}$ & 8 \\
\hline ECV & $\begin{array}{lrr}\text { De reciente } & \text { comienzo. } & \text { Excluir } \\
\text { arteriosclerosis. }\end{array}$ & 8 \\
\hline Vasculitis & $\begin{array}{l}\text { Ulceración, gangrena, nódulos dolorosos } \\
\text { sensibles, infartos periungueales, } \\
\text { hemorragias en astilla o biopsia o } \\
\text { angiografía que confirme la vasculitis. }\end{array}$ & 8 \\
\hline Miositis & $\begin{array}{l}\text { Debilidad proximal/dolor asociado a } \\
\text { elevación de las CPK/aldolasa o EMG } \\
\text { sugestivo o miositis comprobada por } \\
\text { biopsia. }\end{array}$ & 4 \\
\hline Artritis & $\begin{array}{l}\text { Más de dos articulaciones dolorosas y con } \\
\text { signos inflamatorios }\end{array}$ & 4 \\
\hline $\begin{array}{l}\text { Cilindros } \\
\text { urinarios }\end{array}$ & Cilindros hemáticos o granulosos. & 4 \\
\hline Hematuria & $\begin{array}{l}>5 \text { hematíes / c. Excluir litiasis, infección u } \\
\text { otras causas. }\end{array}$ & 4 \\
\hline Proteinuria & $\begin{array}{l}>5 \mathrm{~g} / 24 \mathrm{~h} \text {. De reciente comienzo o aumento } \\
\text { de la proteinuria ya conocida en más de } 0.5 \\
\mathrm{~g} / 24 \mathrm{~h} \text {. }\end{array}$ & 4 \\
\hline Piuria & > 5 leucocitos/c. Excluir infección. & 4 \\
\hline $\begin{array}{l}\text { Exantema } \\
\text { nuevo }\end{array}$ & $\begin{array}{l}\text { Comienzo reciente o recurrente. Exantema } \\
\text { inflamatorio }\end{array}$ & 2 \\
\hline Alopecia & $\begin{array}{l}\text { De comienzo reciente o recurrente. Pérdida } \\
\text { difusa o en placas. }\end{array}$ & 2 \\
\hline Úlceras bucales & $\begin{array}{l}\text { De comienzo reciente o recurrente. Úlceras } \\
\text { bucales o nasales. }\end{array}$ & 2 \\
\hline Pleuritis & $\begin{array}{l}\text { Dolor pleurítico con roce o derrame, o } \\
\text { engrosamiento pleural. }\end{array}$ & 2 \\
\hline Pericarditis & $\begin{array}{l}\text { Dolor pericárdico con al menos uno de } \\
\text { los siguientes: roce, derrame, cambios } \\
\text { electrocardiográficos o confirmación } \\
\text { ecocardiográfica. }\end{array}$ & 2 \\
\hline
\end{tabular}




\begin{tabular}{|c|l|c|}
\hline Complemento & $\begin{array}{l}\text { Descenso de CH50, C3, C4 por debajo del } \\
\text { límite inferior del laboratorio. }\end{array}$ & 2 \\
\hline Anti DNA & $\begin{array}{l}>25 \% . \text { Técnica de Farr o por encima del } \\
\text { valor habitual del laboratorio. }\end{array}$ & 2 \\
\hline Fiebre & $>38^{\circ} \mathrm{C}$. Excluir infección. & 1 \\
\hline Trombopenia & $<100.000$ plaquetas/mm3. & 1 \\
\hline Leucopenia & $<3.000$ células/mm3. Excluir fármacos. & 1 \\
\hline
\end{tabular}

\section{Parámetros}

\section{PUNTAJE ACITIVIDAD DE LA ENFERMEDAD}

\begin{tabular}{|l|l|}
\hline $0-2$ & Inactividad \\
\hline $2-4$ & Leve \\
\hline $4-8$ & Moderada \\
\hline$>8$ & Severa \\
\hline
\end{tabular}

\section{Bibloigrafía}

\section{Original/Primaria}

1. Gladman DD, Ibañez D, Urowitz MB. Índice de actividad de la enfermedad sistémica de lupus eritematoso. J Rheumatol [Internet]. 2002 [citado marzo 2019]; 29 (2): 288-91. Disponible en: http: / / www. jrheum.org/content/29/2/288.long .

\section{Validación}

1. Yee CS, Farewell VT, Isenberg DA, et al. El uso del Índice de Actividad de la Enfermedad del Lupus Eritematoso Sistémico-2000 para definir la enfermedad activa y un cambio mínimo clínicamente significativo basado en datos de una gran cohorte de pacientes con lupus eritematoso sistémico. Reumatología (Oxford) [Internet]. 2011 [citado marzo 2019]; 50 (5): 982-8. Disponible: https: / / www.ncbi.nlm. nih.gov/pubmed/21245073 . 
CÁCULOSY

PRONÓSTICAS

EN MEDICINA INTERNA 


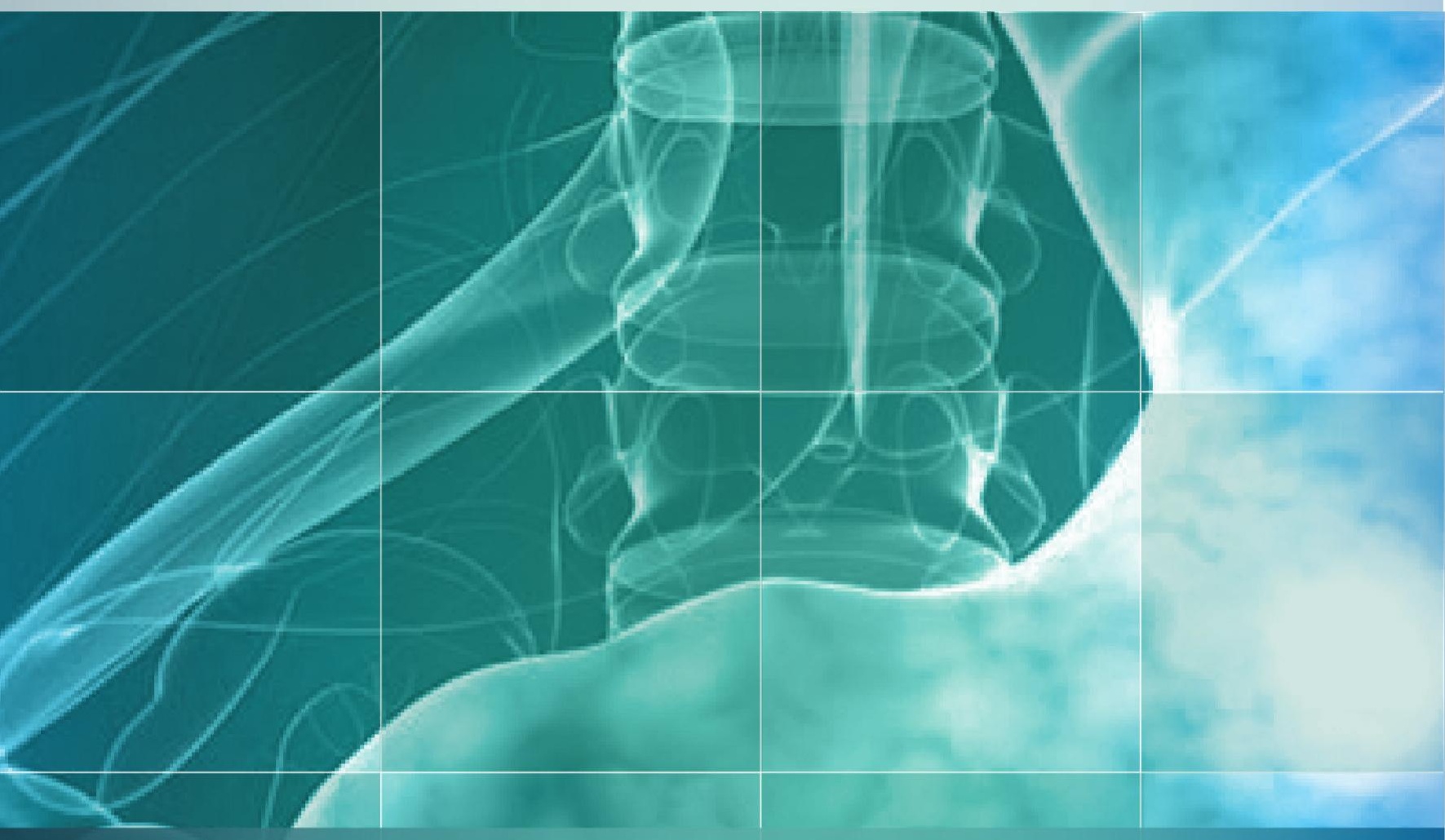

\section{GASTROENTEROLOGÍA}

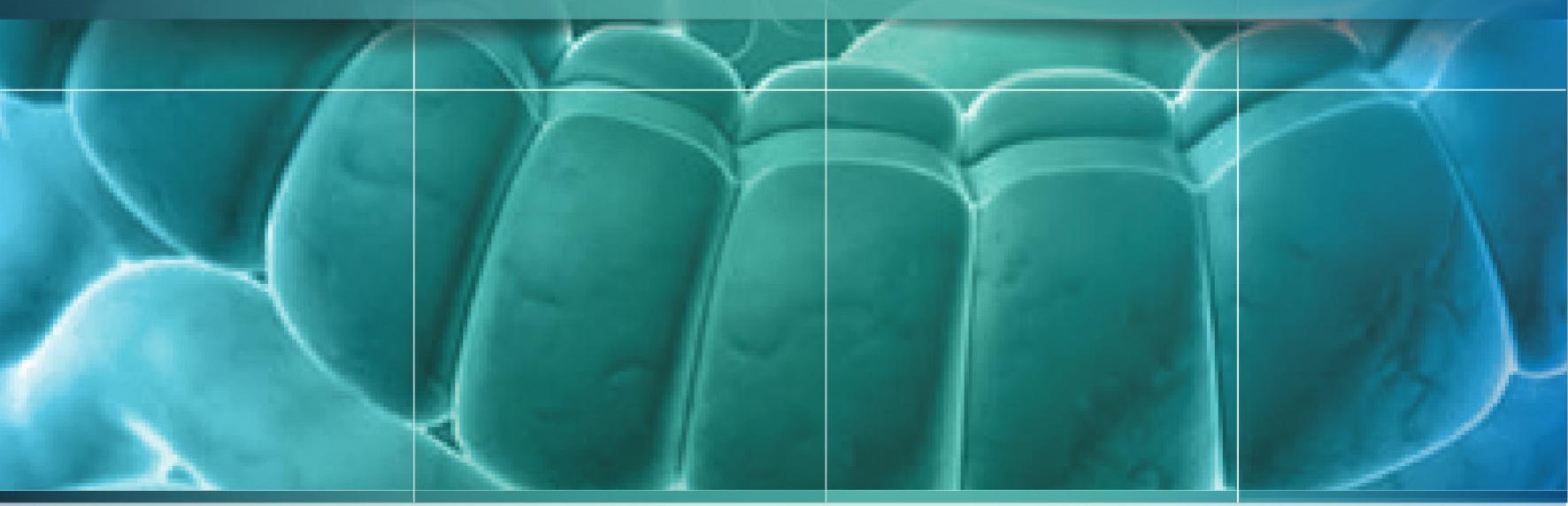




\section{SANGRADO DIGESTIVO}

\section{ESCALA DE BLATCHFORD}

\section{Utilidad}

Estratifica los pacientes con sangrado digestivo alto que son de bajo riesgo y son candidatos para el tratamiento ambulatorio.

\section{Descripción}

- Usar en pacientes adultos considerados para ingreso hospitalario debido a sangrado digestivo superior.

- No usar en pacientes pediátricos o en pacientes con sospecha de hemorragia de intestino delgado o sangrado digestivo bajo.

- Los puntajes varían de 0 a 23, con puntajes más altos correspondientes al aumento de la agudeza y la mortalidad.

\section{Parámetros}

\begin{tabular}{|l|l|c|}
\hline CRITERIO & CARACTERÍSTICAS & PUNTAJE \\
\hline \multirow{5}{*}{ Presión sistólica } & $\geq 110$ & 0 \\
& $100-109 \mathrm{mmHg}$ & 1 \\
& $90-99 \mathrm{mmHg}$ & 2 \\
& $<90 \mathrm{mmHg}$ & 3 \\
\hline \multirow{3}{*}{ Nitrógeno ureico } & $<18.2 \mathrm{mg} / \mathrm{dl}$ & 0 \\
& $18.22-22.3 \mathrm{mg} / \mathrm{dl}$ & 2 \\
& $22.4-28 \mathrm{mg} / \mathrm{dl}$ & 3 \\
& $28-70 \mathrm{mg} / \mathrm{dl}$ & 4 \\
& $>70 \mathrm{mg} / \mathrm{dl}$ & 6 \\
\hline
\end{tabular}




\begin{tabular}{|c|c|c|}
\hline Hemoglobina para hombre & $\begin{array}{l}12.0-12.9 \mathrm{~g} / \mathrm{dl} \\
10.0-11.9 \mathrm{~g} / \mathrm{dl} \\
<10.0 \mathrm{~g} / \mathrm{dl}\end{array}$ & 6 \\
\hline Hemoglobina para mujer & $\begin{array}{l}10.0-11.9 \mathrm{~g} / \mathrm{dl} \\
<10.0 \mathrm{~g} / \mathrm{dl}\end{array}$ & 1 \\
\hline $\begin{array}{l}\text { Otras variables de } \\
\text { presentación }\end{array}$ & $\begin{array}{l}\text { Pulso }>100 \\
\text { Melena } \\
\text { Síncope } \\
\text { Enfermedad hepática } \\
\text { Fallo cardíaco }\end{array}$ & 1 \\
\hline
\end{tabular}

\section{Interpretación}

\section{PUNTUACIÓN INTERPRETACIÓN}

\begin{tabular}{|l|l|}
\hline $\mathbf{0}$ & Bajo riesgo \\
\hline$\geq \mathbf{1}$ & $\begin{array}{l}\text { Cualquier puntuación superior a 0 indica alto } \\
\text { riesgo de necesitar una intervención: transfusión, } \\
\text { endoscopia o cirugía }\end{array}$ \\
\hline
\end{tabular}

\section{Bibliografía}

\section{Original /Primaria}

Blatchford O, Murray W, Blatchford M. Una puntuación de riesgo para predecir la necesidad de tratamiento para la hemorragia uppergastrointestinal. Lancet [Internet]. 2000 [citado marzo 2019]. 356 (9238):1318-1321. Disponible en: https: / / www.thelancet.com/journals/ lancet/ article / PIIS0140-6736\%2800\%2902816-6/ fulltext .

\section{Validación}

1. Chen IC, Hung MS, Chiu TF, Chen JC, Hsiao CT. Sistemas de puntuación de riesgo para predecir la necesidad de intervención 
clínica en pacientes con sangrado del tracto gastrointestinal superior no variceal. Am J Emerg Med [Internet]. Septiembre 2007 [citado marzo 2019]; 25 (7): 774-9. Disponible en: https: / / www.ncbi.nlm.nih. gov / pubmed / 17870480

2. Stanley AJ, et. Alabama. Manejo ambulatorio de pacientes con hemorragia digestiva alta de bajo riesgo: validación multicéntrica y evaluación prospectiva. Lancet \{Internet\}. 2009 [citado marzo 2019]; 373 (9657): 42-7. Disponible en: https:// www.thelancet.com/ journals / lancet/ article/ PIIS0140-6736(08)61769-9/ fulltext\#

3. Srygley FD, Gerardo CJ, Tran T. ¿Este paciente tiene una hemorragia gastrointestinal alta severa?. JAMA [Internet]. 2012 [citado marzo 2019]. ; 307 (10): 1072-1079. Disponible en: https: / / jamanetwork.com/ journals/jama/article-abstract/1105075 . 


\section{Utilidad}

Escala clínica Rockall. Determina la severidad del sangrado gastrointestinal, antes de la endoscopia.

Escala de Rockall completa. Determina la severidad del sangrado gastrointestinal en aquellos pacientes a los que ya se ha realizado una endoscopia

\section{Descripción}

- El puntaje de Rockall completo estima la mortalidad en pacientes con sangrado digestivo alto activo que se han realizado una endoscopia.

- Utilice la puntuación de Rockall previa a la endoscopia para pacientes con sangrado digestivo alto superior que no se hayan realizado una endoscopia.

\section{Parámetros}

\begin{tabular}{|c|c|c|c|c|}
\hline \multirow{6}{*}{ 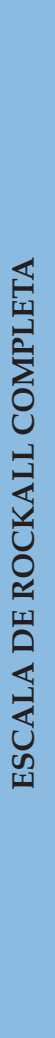 } & \multirow{4}{*}{ 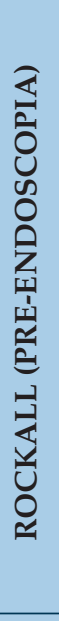 } & CRITERIO & CARACTERÍSTICAS & PUNTAJE \\
\hline & & Edad & $\begin{array}{l}<60 \text { años } \\
60-79 \text { años } \\
\geq 80 \text { años }\end{array}$ & $\begin{array}{l}0 \\
1 \\
2\end{array}$ \\
\hline & & Shock & $\begin{array}{l}\text { Frecuencia cardíaca }>100 \text { latidos / minuto } \\
\text { Presión sistólica }<100 \mathrm{mmHg}\end{array}$ & $\begin{array}{l}1 \\
2\end{array}$ \\
\hline & & $\begin{array}{l}\text { Enfermedades } \\
\text { coexistentes }\end{array}$ & $\begin{array}{l}\text { Enfermedad cardíaca congestiva, } \\
\text { isquémica; falla cardíaca, otras } \\
\text { enfermedades mayores. } \\
\text { Falla renal, falla hepática, cáncer con } \\
\text { metástasis. }\end{array}$ & 2 \\
\hline & & $\begin{array}{l}\text { Diagnóstico } \\
\text { endoscópico }\end{array}$ & $\begin{array}{l}\text { No lesión observada, Mallory Weiss. } \\
\text { Úlcera péptica, enfermedad erosiva, } \\
\text { esofagitis. } \\
\text { Cáncer del trato gastrointestinal superior. }\end{array}$ & $\begin{array}{l}0 \\
1 \\
2\end{array}$ \\
\hline & & $\begin{array}{l}\text { Estigma } \\
\text { endoscópico } \\
\text { de hemorragia } \\
\text { reciente }\end{array}$ & $\begin{array}{l}\text { Base de úlcera limpia, lugar plano } \\
\text { pigmentado. } \\
\text { Sangre en tracto gastrointestinal alto, } \\
\text { sangrado activo, vaso visible, coágulo. }\end{array}$ & $\begin{array}{l}0 \\
2\end{array}$ \\
\hline
\end{tabular}




\section{Interpretación}

\begin{tabular}{|c|c|c|c|}
\hline PUNTUACIÓN & INTERPRETACIÓN & $\begin{array}{c}\text { RIESGO DE NUEVA } \\
\text { HEMORRAGIA }\end{array}$ & MORTALIDAD \\
\hline 0 & \multirow{3}{*}{ Leve (ambulatorio) } & $4.9 \%$ & $0 \%$ \\
\hline 1 & & $3.4 \%$ & $0 \%$ \\
\hline 2 & & $5.3 \%$ & $0.2 \%$ \\
\hline 3 & \multirow{2}{*}{$\begin{array}{c}\text { Moderado } \\
\text { (hospitalización) }\end{array}$} & $11.22 \%$ & $2.9 \%$ \\
\hline 4 & & $14.1 \%$ & $5.3 \%$ \\
\hline 5 & \multirow{4}{*}{ Severo (UCI) } & $24.1 \%$ & $10.8 \%$ \\
\hline 6 & & $32.9 \%$ & $17.3 \%$ \\
\hline 7 & & $43.8 \%$ & $27 \%$ \\
\hline$\geq 8$ & & $41.8 \%$ & $41.1 \%$ \\
\hline
\end{tabular}

\section{Bibliografía}

\section{Original}

1. Rockall TA, Logan RF, Devlin HB, Northfield TC. Evaluación del riesgo tras hemorragia gastrointestinal alta aguda. Gut [Internet]. Marzo 1996 [citado marzo 2019]; 38 (3): 316-21. Disponible en: https: / www. ncbi.nlm.nih.gov/pubmed / 8675081 .

\section{Validación}

1. Kim BJ, Park MK, Kim SJ, Kim ER, Min BH, Hijo HJ. Comparación de los sistemas de puntuación para la predicción de resultados en pacientes con hemorragia del tracto gastrointestinal no variceal: un estudio prospectivo. Dig Dis Sci [Internet]. 2009 Noviembre [citado marzo 2019]; 44: 331-335. Disponible en: https:/ / www.ncbi.nlm.nih. gov/pubmed/19104934.

2. Vreeburg EM, Terwee CB, et. Alabama. Validación del sistema de puntuación de riesgo de Rockall en el sangrado gastrointestinal superior. Gut [Internet]. 1999; 44: 331-335. Disponible en: https:// www.ncbi.nlm.nih.gov/pubmed/19104934. 
CLASIFICACIÓN DE FORREST PARA HEMORRAGIA DIGESTIVA ALTA

\section{Utilidad}

Estratifica la gravedad de la hemorragia GI superior según los hallazgos endoscópicos.

\section{Descripción}

- Sistema de clasificación estandarizado para endoscopistas para describir úlceras pépticas.

- Fiable estratifica a los pacientes con úlceras pépticas y predice el riesgo de nuevas hemorragias y/o mortalidad.

\section{Parámetros}

\begin{tabular}{|l|c|}
\hline \multicolumn{1}{|c|}{ CRITERIO } & CLASE \\
\hline Chorreo activo & $1 \mathrm{~A}$ \\
\hline Supuración activa & $1 \mathrm{~B}$ \\
\hline Vaso visible no sangrante & $2 \mathrm{~A}$ \\
\hline Coágulo adherente & $2 \mathrm{~B}$ \\
\hline Mancha pigmentada plana & $2 \mathrm{C}$ \\
\hline Úlcera con base limpia & 3 \\
\hline
\end{tabular}

\section{Interpretación}

\begin{tabular}{|c|c|c|}
\hline CLASE & TASA DE NUEVAS HEMORRAGIAS & MORTALIDAD \\
\hline $\mathbf{1 A}$ & $55 \%$ & $11 \%$ \\
\hline $\mathbf{1 B}$ & $55 \%$ & $11 \%$ \\
\hline $\mathbf{2 A}$ & $43 \%$ & $11 \%$ \\
\hline $\mathbf{2 B}$ & $22 \%$ & $7 \%$ \\
\hline $\mathbf{2 C}$ & $10 \%$ & $3 \%$ \\
\hline 3 & $5 \%$ & $2 \%$ \\
\hline
\end{tabular}




\section{Bibliografía}

\section{Original /Primaria}

1. Forrest JA, Finlayson ND, Shearman DJ. Endoscopia en hemorragia gastrointestinal. Lancet [Internet]. 1974 [citado marzo 2019]; 2 (7877): 394-7. Disponible en: https://www.ncbi.nlm.nih.gov/ pubmed / 4136718 .

\section{Validación}

1. de Groot NL, van Oijen MG et al. Reevaluación del valor predictivo de la clasificación de Forrest para nuevas hemorragias y mortalidad por úlcera péptica: ¿se puede simplificar la clasificación? Endoscopy [Internet]. 2014 [citado marzo 2019];46 (1): 46-52. Disponible en: https:/ / www.ncbi.nlm.nih.gov/pubmed/24218308 . 


\section{PUNTUACIÓN AIMS65 PARA MORTALIDAD POR HEMORRAGIA DIGESTIVA ALTA}

\section{Utilidad}

La AIMS65, determina el riesgo de mortalidad hospitalaria por sangrado digestivo alto.

\section{Descripción}

- Utiliza la información disponible antes de la endoscopia.

- La mortalidad aumenta con cada factor de riesgo positivo.

- El estado mental alterado se define como la puntuación de la escala de coma de Glasgow < 14 o la designación de un médico como: desorientado, letargo, estupor o coma.

\section{Parámetros}

\begin{tabular}{|c|l|c|}
\hline & \multicolumn{1}{|c|}{ CRITERIO } & PUNTAJE \\
\hline $\mathbf{A}$ & Albúmina $<3 \mathrm{~g} / \mathrm{dL}$ & 1 \\
\hline I & INR $>1.5$ & 1 \\
\hline $\mathbf{M}$ & Alteración en el estado mental & 1 \\
\hline $\mathbf{S}$ & Presión arterial sistólica $\leq 90 \mathrm{~mm}$ hg & 1 \\
\hline 65 & Edad $\geq 65$ años & 1 \\
\hline
\end{tabular}

\section{Interpretación}

\section{PUNTUACIÓN $\quad$ TASA DE MORTALIDAD HOSPITALARIA}

\begin{tabular}{|l|c|}
\hline $\mathbf{0}$ & $0.3 \%$ \\
\hline $\mathbf{1}$ & $1.2 \%$ \\
\hline $\mathbf{2}$ & $5.3 \%$ \\
\hline $\mathbf{3}$ & $10.3 \%$ \\
\hline $\mathbf{4}$ & $16.5 \%$ \\
\hline $\mathbf{5}$ & $24.5 \%$ \\
\hline
\end{tabular}




\section{Bibliografía}

\section{Original}

1. Saltzman JR, Tabak YP, Hyett BH, Sun X, Travis AC, Johannes RS. Una simple puntuación de riesgo predice con precisión la mortalidad hospitalaria, la duración de la estancia y el costo del sangrado GI superior agudo. Gastrointest Endosc. 2011 [citado 27 marzo 2019]; 74: 1215-1224. Disponible en: https://www.ncbi.nlm.nih.gov/ pubmed $/ 21907980$.

\section{Validación}

1. Thandassery RB, Sharma M, John AK, et al. Aplicación clínica de las puntuaciones AIMS65 para predecir resultados en pacientes con hemorragia gastrointestinal superior. Clin Endosc. 2015 [citado 27 marzo 2019]; 48 (5): 380-384. Disponible en: https:/ / www.ncbi.nlm. nih.gov/pmc/articles/PMC4604275/ .

2. Stanley AJ, Laine L, Dalton HR, Ngu JH, Schultz M, Abazi R, et al. Comparación de los sistemas de puntuación de riesgo para pacientes con hemorragia digestiva alta: estudio prospectivo internacional multicéntrico. BMJ [Internet]. Enero 2017 [citado marzo 21019];356:i6432. Disponible en: https://www.ncbi.nlm.nih.gov/ pubmed / 28053181.

3. Gaduputi V, Abdulsamad M, Tariq H, Rafeeq A, Abbas N, Kumbum K, Chilimuri S. Valor pronóstico de la puntuación AIMS65 en pacientes cirróticos con sangrado gastrointestinal superior. IGastroenterol Res Pract [Internet]. 2014 [citado marzo 2019]; : 787256. Disponible en: https: / / www.ncbi.nlm.nih.gov/pmc/articles / PMC4283417/ .

4. Yaka E, Yılmaz S, Özgür Doğan N, Pekdemir M. Comparación de los sistemas de puntuación de Glasgow-Blatchford y AIMS65 para la estratificación del riesgo en el sangrado gastrointestinal superior en el departamento de emergencias. Medicina académica de emergencia [Internet]. 2014 [citado marzo 2019]: 22(1). Disponible en: https:// onlinelibrary.wiley.com/doi / full/10.1111/acem.12554. 


\section{CIRROSIS HEPÁTICA}

\section{ESCALA DE CHILD-PUGH}

\section{Utilidad}

Es un sistema de estadificación para evaluar la severidad de la cirrosis hepática.

\section{Descripción}

- Aunque originalmente se usó para predecir la probable mortalidad asociada a la cirugía, se usa ahora para determinar el pronóstico, así como la intensidad requerida por el paciente en su tratamiento y la necesidad de un trasplante de hígado.

\section{Parámetros}

\begin{tabular}{|c|c|c|}
\hline CRITERIO & CARACTERÍSTICAS & PUNTAJE \\
\hline Bilirrubina total & $\begin{array}{l}<2 \mathrm{mg} / \mathrm{dL}(<34.2 \mu \mathrm{mol} / \mathrm{L}) \\
2-3 \mathrm{mg} / \mathrm{dL}(34.2-51.3 \mu \mathrm{mol} / \mathrm{L}) \\
>3 \mathrm{mg} / \mathrm{dL}(>51.3 \mu \mathrm{mol} / \mathrm{L})\end{array}$ & $\begin{array}{l}1 \\
2 \\
3\end{array}$ \\
\hline Albúmina sérica & $\begin{array}{l}>3.5 \mathrm{~g} / \mathrm{dL}(>35 \mathrm{~g} / \mathrm{L}) \\
2.8-3.5 \mathrm{~g} / \mathrm{dL}(28-35 \mathrm{~g} / \mathrm{L}) \\
<2.8 \mathrm{~g} / \mathrm{dL}(<28 \mathrm{~g} / \mathrm{L})\end{array}$ & $\begin{array}{l}1 \\
2 \\
3\end{array}$ \\
\hline $\begin{array}{l}\text { INR/Tiempo de } \\
\text { protrombina }\end{array}$ & $\begin{array}{l}<1.7 />50 \\
1.7-2.3 / 30-50 \\
>2.3 /<30\end{array}$ & $\begin{array}{l}1 \\
2 \\
3\end{array}$ \\
\hline Ascitis & $\begin{array}{l}\text { Ausente } \\
\text { Leve } \\
\text { Moderada - severa (refractaria) }\end{array}$ & $\begin{array}{l}1 \\
2 \\
3\end{array}$ \\
\hline
\end{tabular}




\begin{tabular}{|l|l|c|}
\hline $\begin{array}{l}\text { Encefalopatía } \\
\text { hepática }\end{array}$ & Ausente & 1 \\
& Grado I-II & 2 \\
\hline
\end{tabular}

\section{Interpretación}

\begin{tabular}{|c|c|c|c|c|}
\hline Puntuación & Clase & $\begin{array}{c}\text { Supervivencia } \\
\text { al cabo de 1 año }\end{array}$ & $\begin{array}{c}\text { Supervivencia al } \\
\text { cabo de 2 años }\end{array}$ & \\
\hline $\mathbf{5 - 6}$ & A & $100 \%$ & $85 \%$ & $\begin{array}{c}\text { Enfermedad bien } \\
\text { compensada. }\end{array}$ \\
\hline $\mathbf{7 - 9}$ & B & $81 \%$ & $57 \%$ & $\begin{array}{c}\text { Compromiso } \\
\text { funcional } \\
\text { significativo. }\end{array}$ \\
\hline $\mathbf{1 0 - 1 5}$ & $\mathrm{C}$ & $45 \%$ & $35 \%$ & $\begin{array}{c}\text { Enfermedad } \\
\text { descompensada. }\end{array}$ \\
\hline
\end{tabular}

\section{Bibliografía}

\section{Original/Primaria}

1. Child CG, Turcotte JG. Surgery and portal hypertension. In: The liver and portal hypertension. Edited by CG Child. Philadelphia: Saunders 1964:50-64.

\section{Validación}

1. Pugh RN, Murray-Lyon IM, Dawson JL, Pietroni MC, Williams R. Transection of the oesophagus for bleeding oesophageal varices. Br J Surg [Internet]. 1973 [citado marzo 2019]. 60 (8). Disponible en: https: / / www.ncbi.nlm.nih.gov/pubmed/4541913 . 


\section{PUNTUACIÓN MELD}

\section{Utilidad}

El score MELD es utilizado para estimar la severidad de los pacientes con cirrosis hepática y la tasa de supervivencia de quienes esperan un trasplante hepático. Útil en pacientes mayores a 12 años.

\section{Descripción}

- Los valores no deben tener más de 48 horas de antigüedad.

- La puntuación MELD se debe reevaluar periódicamente, ya que varia con los cambios en los valores de laboratorio.

\section{Parámetros}

$\begin{array}{ll}\text { Creatinina } & \mathrm{mg} / \mathrm{dl} \\ \text { Bilirrubina } & \mathrm{mg} / \mathrm{dl} \\ \text { INR } & \end{array}$

\section{Fórmula}

MELD Score $=9.57 \times \operatorname{Ln}($ Creatinina en suero $)+3.78 \times \operatorname{Ln}($ Bilirrubina sérica $)+11,2 \times$ Ln $($ INR) +6.43

\section{Interpretación}

\begin{tabular}{|c|c|}
\hline PUNTUACIÓN & MORTALIDAD A LOS 3 MESES \\
\hline $\mathbf{4 0}$ o más & $71.3 \%$ \\
\hline $\mathbf{3 0 - 3 9}$ & $52.6 \%$ \\
\hline $\mathbf{2 0 - 2 9}$ & $19.6 \%$ \\
\hline $\mathbf{1 0 - 1 9}$ & $6 \%$ \\
\hline$<9$ & $1.9 \%$ \\
\hline
\end{tabular}




\section{Bibliografía}

\section{Original}

1. Kamath PS, Wiesner RH, Malinchoc M, Kremers W, Therneau TM, Kosberg CL, D'Amico G, Dickson ER, Kim WR. Un modelo para predecir la supervivencia en pacientes con enfermedad hepática terminal. Hepatology [Internet]. 2001 Feb [citado marzo 2019]; 33 (2): 464-70. Disponible en: https://www.ncbi.nlm.nih.gov/ pubmed / 11172350 .

\section{Validación}

1. Wiesner R, Comité de puntuación de gravedad de gravedad de enfermedad hepática de órganos compartidos de órganos, y otros Modelo para enfermedad hepática en etapa terminal (MELD, por sus siglas en inglés) y asignación de hígados de donantes. Gastroenterology [Internet]. 2003 Ene [citado marzo 2019]; 124 (1): 91-6. Disponible en: https:// www.ncbi.nlm.nih.gov/pubmed/12512033.

2. Kremers WK, van IJperen M, Kim WR, Freeman RB, Harper AM, Kamath PS, Wiesner RH. La puntuación MELD como predictor de la supervivencia antes y después del trasplante en pacientes OPTN / UNOS de estado 1. Hepatología [Internet]. 2004 [citado marzo 2019]; 39 (3): 764-9. Disponible en: https://www.ncbi.nlm.nih.gov/ pubmed/14999695. 


\section{PANCREATITIS AGUDA}

CRITERIOS GLASGOW- IMRIE

\section{Utilidad}

Es un sistema que determina la severidad de la pancreatitis en base a 8 valores de laboratorio.

\section{Descripción}

- Estos criterios se califican tradicionalmente con valores, a las 48 horas después del ingreso.

\section{Parámetros}

\begin{tabular}{|l|l|c|}
\hline CRITERIO & CARACTERÍSTICAS & PUNTAJE \\
\hline $\mathbf{P a O}_{2}$ & $<59.3 \mathrm{mmHg}$ & 1 \\
\hline Edad & $>55 \mathrm{años}$ & 1 \\
\hline Glóbulos blancos & $>15 \times 10^{3} / \mu \mathrm{L}\left(10^{9} / \mathrm{L}\right)$ & 1 \\
\hline Calcio & $<8 \mathrm{mg} / \mathrm{dL}(2 \mathrm{mmol} / \mathrm{L})$ & 1 \\
\hline BUN & $>44.8 \mathrm{mg} / \mathrm{dL}$ & 1 \\
\hline LDH & $>600 \mathrm{UI} / \mathrm{L}$ & 1 \\
\hline Albúmina & $<3.2 \mathrm{~g} / \mathrm{dL}(32 \mathrm{~g} / \mathrm{L})$ & 1 \\
\hline Glucosa & $>180 \mathrm{mg} / \mathrm{dL}(10 \mathrm{mmol} / \mathrm{L})$ & 1 \\
\hline
\end{tabular}

Interpretación

PUNTUACIÓN

\begin{tabular}{|c|c|}
\hline $\mathbf{0}$ & $7 \%$ \\
\hline $\mathbf{1}$ & $6 \%$ \\
\hline $\mathbf{2}$ & $16 \%$ \\
\hline 3 & $20 \%$ \\
\hline
\end{tabular}




\begin{tabular}{|c|c|}
\hline $\mathbf{4}$ & $61 \%$ \\
\hline $\mathbf{5}$ & $55 \%$ \\
\hline $\mathbf{6}$ & $100 \%$ \\
\hline $\mathbf{7}$ & $100 \%$ \\
\hline $\mathbf{8}$ & $100 \%$ \\
\hline
\end{tabular}

\section{Bibliografía}

\section{Original}

Blamey SL, Imrie CW, O'Neill J, Gilmour WH, Carter DC. Factores pronósticos en la pancreatitis aguda. Gut [Internet]. Diciembre 1984 [citado marzo 2019]; 25 (12): 1340-6. Disponible en: https:/ / www.ncbi. nlm.nih.gov/pmc/articles/PMC1420197/pdf/gut00397-0014.pdf .

\section{Validación}

Mounzer R, Langmead CJ, Wu BU, et al. Comparación de los sistemas de puntuación clínica existentes para predecir la insuficiencia orgánica persistente en pacientes con pancreatitis aguda. Gastroenterology. 2012 Jun [citado 27 marzo 2019]; 142 (7): 1476-82. Disponible en: https: / www. ncbi.nlm.nih.gov/pubmed/22425589 .

Barreto SG; Rodrigues J. Comparación de APACHE II e Imrie Scoring Systems en la predicción de la gravedad de la pancreatitis aguda. World J Emerg Surg [Internet]. 2007 [citado marzo 2019] ; 2: 33. Disponible en: https: / / www.ncbi.nlm.nih.gov/pmc/articles/PMC2169219/ . 


\section{CRITERIOS DE RANSON}

\section{Utilidad}

Estima la mortalidad de los pacientes con pancreatitis, según los valores de laboratorio iniciales y de 48 horas.

\section{Descripción}

Ayuda a determinar la disposición del paciente, con una puntuación más alta correspondiente a un nivel más alto de atención.

\section{Parámetros}

\begin{tabular}{|l|l|c|}
\hline \multicolumn{3}{|c|}{ EN LA ADMISIÓN } \\
\hline \multicolumn{1}{|c|}{ CRITERIO } & \multicolumn{1}{|c|}{ CARACTERÍSTICAS } & PUNTAJE \\
\hline Edad & $>55 \mathrm{años}$ & 1 \\
\hline Leucocitos & $>16000 \mathrm{~mm}^{3}$ & 1 \\
\hline Glucemia & $>200 \mathrm{mg} / \mathrm{dl}$ & 1 \\
\hline AST & $>250 \mathrm{U} / \mathrm{L}$ & 1 \\
\hline LDH & $>350 \mathrm{U} / \mathrm{L}$ & 1 \\
\hline 48 HORAS DESPUÉS & 1 \\
\hline Hematocrito & caída de $>10 \%$ & 1 \\
\hline BUN & $\begin{array}{l}\text { Incremento de } 5 \mathrm{mg} / \mathrm{dl} \text { a pesar de } \\
\text { líquidos }\end{array}$ & 1 \\
\hline Calcio en suero & $<8 \mathrm{mg} / \mathrm{dl}$ & 1 \\
\hline PO ${ }_{2}$ & $>60 \mathrm{mmHg}$ & 1 \\
\hline Déficit de base & $>4 \mathrm{mEq} / 1$ & 1 \\
\hline $\begin{array}{l}\text { Secuestro de } \\
\text { líquidos }\end{array}$ & $>6000 \mathrm{ml}$ & \\
\hline
\end{tabular}




\section{Interpretación}

\section{PUNTUACIÓN interpretación}

\begin{tabular}{|l|l|}
\hline $0-2$ & Pancreatitis leve, 1\% mortalidad \\
\hline $3-6$ & Pancreatitis moderada, 16\% mortalidad \\
\hline$>6$ & Pancreatitis severa, $>40 \%$ mortalidad \\
\hline
\end{tabular}

\section{Bibliografía}

\section{Original}

1. Ranson JH, Rifkind KM, Roses DF, Fink SD, Ing. K, Spencer FC. Signos pronósticos y el papel del manejo quirúrgico en la pancreatitis aguda. Surg Gynecol Obstet [Internet]. Julio 1974 [citado marzo 2019]; 139 (1): 69-81. Disponible en: https: / / www.ncbi.nlm.nih.gov / pubmed $/ 4834279$. 


\section{SCORE BISAP}

\section{Utilidad}

Predice el riesgo de mortalidad en pancreatitis, utiliza menos variables que los criterios de Ranson.

\section{Descripción}

- Los datos deben tomarse de las primeras 24 horas de la evaluación del paciente.

- El score BISAP es un puntaje más reciente que los criterios de Ranson, y no requiere puntos de datos de 48 horas, desde la admisión al hospital de un paciente.

\section{Parámetros}

\begin{tabular}{|c|c|c|}
\hline & CRITERIO & PUNTAJE \\
\hline BUN & BUN $>25 \mathrm{mg} / \mathrm{dL}(8.92 \mathrm{mmol} / \mathrm{L})$ & 1 \\
\hline $\begin{array}{c}\text { Impaired } \\
\text { mental } \\
\text { status }\end{array}$ & $\begin{array}{c}\text { Estado mental deteriorado, definido como } \\
\text { desorientación, letargo, somnolencia, coma } \\
\text { o estupor. }\end{array}$ & 1 \\
\hline SIRS & $\geq 2$ Criterios SIRS & 1 \\
\hline Age & Edad $>60$ años & 1 \\
\hline $\begin{array}{c}\text { Pleural } \\
\text { effusions }\end{array}$ & Derrame pleural presente & 1 \\
\hline
\end{tabular}

\section{Interpretación}

Se considera a un paciente con pancreatitis severa si tiene un Score BISAP $>0$ igual a 3 . 


\section{Bibliografía}

\section{Original}

1. Wu BU, Johannes RS, Sol X, Tabak Y, Conwell DL, Banks PA. La predicción temprana de la mortalidad en la pancreatitis aguda: un gran estudio de base poblacional. Gut \{Internet\}. 2008 [citado marzo 2019]; 57 (12): 1698-703. Disponible en: https:/ / www.ncbi.nlm.nih. gov/pubmed/18519429.

\section{Validación}

1. Papachristou GI, Muddana V, Yadav D, et al. Comparación de las puntuaciones de BISAP, Ranson, APACHE-II y CTSI en la predicción de insuficiencia orgánica, complicaciones y mortalidad en la pancreatitis aguda. Am J Gastroenterol [Internet]. 2010 [citado marzo 2019]; 105 (2): 435-41. Disponible en: https: / / www.ncbi.nlm.nih.gov / pubmed/19861954 .

2. Gompertz M, Fernández L, Lara I, Miranda JP, Mancilla C, Berger Z. Índice de gravedad en la pancreatitis aguda (BISAP) de cabecera como predictor del resultado clínico en pancreatitis aguda: revisión retrospectiva de 128 pacientes. Rev Med Chil [Internet]. 2012 [citado marzo 2019]; 140 (8): 977-83. Disponible en: https:/ / www.ncbi.nlm. nih.gov/pubmed/23282769. 


\section{ESCALA DE MARSHALL MODIFICADA}

\section{Utilidad}

Es una herramienta utilizada para evaluar la falla multiorgánica en pacientes con pancreatitis aguda.

\section{Parámetros}

\begin{tabular}{|c|c|c|c|c|c|}
\hline CRITERIOS & $\mathbf{0}$ & $\mathbf{1}$ & $\mathbf{2}$ & $\mathbf{3}$ & $\mathbf{4}$ \\
\hline $\begin{array}{c}\text { Respiratorio } \\
\text { (PaO2/FiO2) }\end{array}$ & $>400$ & $301-400$ & $201-300$ & $101-200$ & $<101$ \\
\hline $\begin{array}{c}\text { Renal (creatinina } \\
\text { sérica) }\end{array}$ & $<1.4$ & $1.4-1.8$ & $1.9-3.6$ & $3.6-4.9$ & $>4.9$ \\
\hline $\begin{array}{c}\text { Cardiovascular } \\
\text { (tensión arterial } \\
\text { sistólica, mm Hg) }\end{array}$ & $>90$ & $\begin{array}{c}\text { Responde } \\
\text { a fluidos }\end{array}$ & $\begin{array}{c}\mathrm{No} \\
\text { responde } \\
\text { a fluidos }\end{array}$ & $\mathrm{Ph}<7.3$ & $\begin{array}{c}\mathrm{Ph} \\
<7.2\end{array}$ \\
\hline
\end{tabular}

\section{Interpretación}

\section{INTERPRETACIÓN}

Resultado $\geq 2$ para cualquier sistema supone falla orgánica.

\section{Bibliografía}

\section{Original/Primaria}

3. Marshall JC, Cook DJ, Christou NV, et al. Puntuación de disfunción orgánica múltiple: un descriptor confiable de un resultado clínico complejo. Crit Care Med [Internet] 1995 [citado marzo 2019]; 23: 163852. Disponible en: https: / / read.qxmd.com/read/7587228/multipleorgan-dysfunction-score-a-reliable-descriptor-of-a-complex-clinicaloutcome. 


\section{Validación}

4. Huerta-Mercado J. Tratamiento médico de la pancreatitis aguda. Rev Med Hered [Internet]. 2013 [citado marzo 2019]; 24:231-236. Disponible en: http:/ / www.scielo.org.pe/ pdf/rmh/v24n3/v24n3r1.pdf. 


\section{CRITERIOS TOMOGRÁFICOS DE BALTHAZAR}

\section{Utilidad}

Es un sistema de clasificación de la pancreatitis aguda, basado en la tomografía computarizada que define cinco grados (A, B, C, D y E), tomando en cuenta densidad, volumen, entorno y existencia o no de enfermedad inflamatoria peripancreática acompañada de colecciones líquidas.

Además se acompaña de una puntuación para establecer la presencia y extensión de necrosis parenquimatosa pancreática, entonces en conjunto se les denomina CTSI (indice de gravedad por TC), estima el grado de necrosis y el índice de severidad de la enfermedad.

\section{Parámetros}

\begin{tabular}{|c|l|c|}
\hline CLASIFICACIÓN & CRITERIO & PUNTAJE \\
\hline A & Normal. & 0 \\
\hline B & $\begin{array}{l}\text { Agrandamimento difuso del } \\
\text { páncreas. }\end{array}$ & 1 \\
\hline C & $\begin{array}{l}\text { Anomalías intrínsecas del páncreas } \\
\text { asociado a cambios de tejido } \\
\text { peripandreático. }\end{array}$ & 2 \\
\hline D & $\begin{array}{l}\text { Presencia de una colección mal } \\
\text { definida. }\end{array}$ & 3 \\
\hline E & $\begin{array}{l}\text { Presencia de 2 o mas colecciones } \\
\text { líquidas mal definidas. }\end{array}$ & 4 \\
\hline
\end{tabular}

\section{Interpretación}

\begin{tabular}{|l|l|}
\hline PUNTUAJE & \multicolumn{1}{|c|}{$\begin{array}{c}\text { GRADO DE } \\
\text { NECROSIS }\end{array}$} \\
\hline $\mathbf{0}$ & $0 \%$ \\
\hline $\mathbf{2}$ & $<30 \%$ \\
\hline $\mathbf{4}$ & $30-50 \%$ \\
\hline $\mathbf{6}$ & $>50 \%$ \\
\hline
\end{tabular}

\begin{tabular}{|l|l|}
\hline $\begin{array}{c}\text { SUMA DE } \\
\text { PUNTOS }\end{array}$ & \multicolumn{1}{|c|}{$\begin{array}{c}\text { INDICE DE } \\
\text { SEVERIDAD }\end{array}$} \\
\hline $0-3$ & Bajo \\
\hline $4-6$ & Medio Alto \\
\hline $7-10$ & Alto \\
\hline & \\
\hline
\end{tabular}




\section{Bibliografía}

\section{Original/Primaria}

1. Balthazar EJ, Robinson DL, Megibow AJ, Ranson JHC. Pancreatitis aguda: valor de la TC para establecer el pronóstico. Radiology [Internet]. 1990 [citado marzo 2019]; 174:331-336. Dsiponible en: https: / / www.ncbi.nlm.nih.gov/ pubmed/2296641.

2. Balthazar EJ, Freeny PC, vanSonnenberg E. Imagen e intervención en pancreatitis aguda. Radiology [Internet]. 1994 [citado marzo 2019]; 193 (2): 297-306. Disponible en: https://www.ncbi.nlm.nih. gov / pubmed / 7972730 .

\section{Validación}

1. Paulson EK, Vitellas KM, Keogan MT, Low VH, Nelson RC. Pancreatitis aguda complicada por necrosis de las glándulas: espectro de hallazgos en la TC con contraste. AJR Am J Roentgenol [Internet]. 1999 [citado marzo 2019]. Disponible en: https: / / www.ncbi.nlm.nih. gov/pubmed/10063845.

2. Schwaner J, Rivas F, Cancino A, Torres O. Pancreatitis aguda: indice de severidad en tc. Evaluacion de complicaciones y hospitalización. Rev. Chil. Radiol [Internet]. 2003 [citado marzp 2019]:9(6);187-193 . 


\section{HEPATITIS ALCOHÓLICA}

\section{PUNTAJE ABIC PARA LA HEPATITIS ALCOHÓLICA}

\section{Utilidad}

Se usa como predictor de la supervivencia en pacientes con hepatitis alcohólica.

\section{Parámetros}

Edad

Bilirrubina sérica $\quad \mathrm{mg} / \mathrm{dl}$

INR

Creatina en suero $\quad \mathrm{mg} / \mathrm{dl}$

Fórmula

Puntuación ABIC $=($ Age, años $\times 0.1)+($ Bilirrubina suero, $m g / d l \times 0.08)+($ $I N R \times 0.8)+($ Creatinina suero, $m g / d l \times 0.3)$

\section{Interpretación}

\begin{tabular}{|c|c|c|c|}
\hline $\begin{array}{c}\text { PUNTUACIÓN } \\
\text { ABIC }\end{array}$ & $\begin{array}{c}\text { Grupo de } \\
\text { riesgo }\end{array}$ & $\begin{array}{c}\text { Supervivencia } \\
\mathbf{9 0} \text { días }\end{array}$ & $\begin{array}{c}\text { Supervivencia } \\
\mathbf{1} \text { año }\end{array}$ \\
\hline$<6.71$ & Bajo & $100 \%$ & $97.1 \%$ \\
\hline $\mathbf{6 . 7 1}$ a $<\mathbf{9 . 0 0}$ & Intermedio & $70 \%$ & $64.3 \%$ \\
\hline$\geq \mathbf{9 . 0 0}$ & Alto & $25 \%$ & $33.3 \%$ \\
\hline
\end{tabular}

\section{Bibliografía}

\section{Original/Primaria}

1. Domínguez M, Rincón D, Abraldes JG, et al. Un nuevo sistema de puntuación para la estratificación pronóstica de pacientes con hepatitis alcohólica. Am J Gastroenterol [Internet]. 2008 [citado marzo 2019]; 103 (11): 2747-56. Disponible en: https://www.ncbi.nlm.nih.gov/ pubmed/18721242. 


\section{Validación}

1. Papastergiou V, Tsochatzis EA, Pieri G, et al. Nueve modelos de puntuación para la mortalidad a corto plazo en la hepatitis alcohólica: validación cruzada en una cohorte comprobada mediante biopsia. Aliment Pharmacol Ther [Internet]. 2014 [citado marzo 2019]; 39 (7): 721-32. Disponible en: https://www.mdcalc.com/abic-scorealcoholic-hepatitis\# evidence.

2. Garcia-Saenz-de-Sicilia M, Duvoor C, Altamirano J, Chavez-Araujo R. Un modelo de Lille del día 4 predice la respuesta a los corticosteroides y la mortalidad en la hepatitis alcohólica grave. Am J Gastroenterol [Internet]. 2017 [citado marzo 2019]; 112 (2): 306-315. Disponible en: https: / / www.ncbi.nlm.nih.gov/pubmed/27922027.

3. Lafferty H, Stanley AJ, Forrest EH. La gestión de la hepatitis alcohólica: una comparación prospectiva de los sistemas de puntuación. Aliment Pharmacol Ther [Internet]. 2013 septiembre [citado marzo 2019]; 38 (6): 603-10. Disponible en: https://www.ncbi.nlm.nih.gov/ pubmed / 23879668 . 


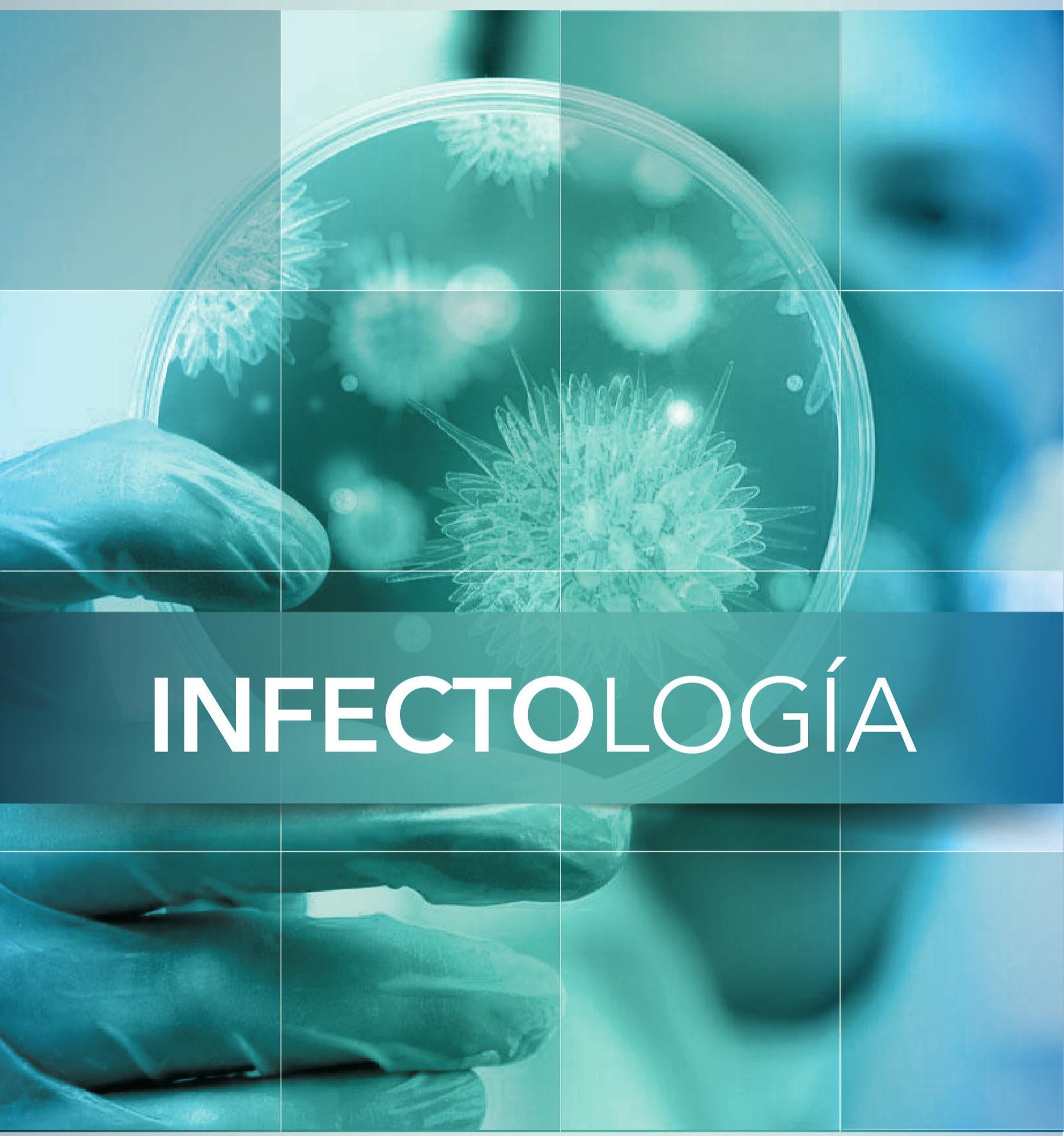


CÁculos $Y$

PROHÓSTICAS

EN MEDICINA INTERNA 


\section{ENDOCARDITIS INFECCIOSA}

\section{CRITERIOS DE DUKE PARA ENDOCARDITIS INFECCIOSA}

\section{Utilidad}

Proporciona criterios diagnósticos para endocarditis infecciosa (EI).

\section{Descripción}

- Se debe sospechar de EI y considerar los criterios de Duke en pacientes con:

- Fiebre prolongada (fiebre de origen desconocido).

- Fiebre y fenómenos vasculares (accidente cerebrovascular, isquemia de las extremidades, hallazgos físicos de los émbolos sépticos).

- Hemocultivos persistentemente positivos (2 o más).

- Válvulas protésicas que son febriles.

- Los usuarios de drogas inyectables que son febriles.

- Una condición cardíaca predisponente que es febril.

- Fiebre con antecedentes recientes de hospitalización.

\section{Parámetros}

\section{CARACTERISTICAS}

\section{CRITERIOS PATOLÓGICOS}

Si alguno de los dos es positivo, el diagnóstico es definitivo

Microorganismos Demostrado por el cultivo o el examen histológico en una vegetación de una vegetación, una vegetación embolizada o una muestra de absceso intracardíaco.

Lesiones patológicas

Vegetación o absceso intracardíaco confirmado por examen histológico que muestra endocarditis activa. 


\section{CRITERIOS MAYORES}

\section{Si ambos son positivos, el diagnóstico es definitivo}

\begin{tabular}{|l|l|}
\hline $\begin{array}{l}\text { Hemocultivos } \\
\text { positivos para } \\
\text { endocarditis }\end{array}$ & $\begin{array}{l}\text { Microorganismos típicos compatibles con EI, } \\
\text { de 2 hemocultivos separados, microorganismos } \\
\text { compatibles con EI de hemocultivos positivos } \\
\text { persistentes, hemocultivo positivo único para } \\
\text { coxiella burnetii o título de anticuerpo antifase I } \\
\text { IgG>1: } 800 .\end{array}$ \\
\hline $\begin{array}{l}\text { Evidencia de } \\
\text { afectación } \\
\text { endocárdica }\end{array}$ & $\begin{array}{l}\text { Ecocardiograma positivo para EI, absceso, nueva } \\
\text { dehiscencia parcial de válvula protésica, nueva } \\
\text { insuficiencia valvular. Nota: El empeoramiento o } \\
\text { el cambio del soplo preexistente no es suficiente. }\end{array}$ \\
\hline
\end{tabular}

\section{CRITERIOS MENORES}

\section{Si todos son positivos, el diagnóstico es definitivo}

Predisposición de afección cardíaca o uso de drogas inyectables

Fiebre

Fenómenos vasculares

Embolias arteriales mayores, infartos pulmonares sépticos, aneurisma micótico, hemorragia intracraneal, hemorragias conjuntivales y lesiones de Janeway.

Fenómenos inmunológicos

Glomerulonefritis, nódulos de osler, manchas de roth y factor reumatoide.

Evidencia microbiológica

El hemocultivo positivo, pero no cumple con el criterio principal como se señaló anteriormente o la evidencia serológica de infección activa con un organismo compatible con IE.

\section{Interpretación}

\begin{tabular}{|l|l|}
\hline \multicolumn{2}{|c|}{ INTERPRETACIÓN } \\
\hline \multirow{4}{*}{ DIAGNÓSTICO POSITIVO } & 1 criterio patológico \\
\cline { 2 - 2 } & 2 criterios mayores \\
\cline { 2 - 2 } & 1 criterio mayor +1 criterio menor \\
\cline { 2 - 2 } & 3 criterios menores \\
\hline
\end{tabular}




\section{Bibliografía}

\section{Original/Primaria}

Durack DT, Lukes AS, Bright DK. Nuevos criterios para el diagnóstico de endocarditis infecciosa: utilización de hallazgos ecocardiográficos específicos. Servicio de endocarditis de duque. Am J Med [Internet]. 1994 marzo [citado marzo 2019]; 96 (3): 200-9. Disponible en: https:/ / www. ncbi.nlm.nih.gov/pubmed/8154507 .

\section{Validación}

Li JS, Sexton DJ, Mick N, Nettles R, Fowler VG Jr, Ryan T, Bashore T, Corey GR. Modificaciones propuestas a los criterios de Duke para el diagnóstico de endocarditis infecciosa. Clin Infect Dis [Internet]. Abril 2000 [citado marzo 2019]; 30 (4): 633-8. Disponible en: https://www.ncbi.nlm.nih. gov/pubmed/10770721. 


\section{SEPSIS}

\section{SOFA (SEQUENTIAL ORGAN FAILURE ASSESSMENT)}

\section{Utilidad}

Predice la mortalidad en la UCI, según los resultados de laboratorio y los datos clínicos.

\section{Descripción}

- El puntaje se calcula al ingreso y cada 24 horas hasta el alta usando los peores parámetros medidos durante las 24 horas anteriores.

\section{Parámetros}

\begin{tabular}{|c|c|c|c|c|c|c|c|}
\hline \multirow{2}{*}{ CRITERIOS } & \multicolumn{7}{|c|}{ PUNTAJE } \\
\hline & \multirow[t]{2}{*}{0} & 1 & \multirow{2}{*}{2} & 3 & \multicolumn{3}{|l|}{4} \\
\hline Respiratorio & & & & & & & \\
\hline $\mathrm{PaO}_{2} / \mathrm{FiO}_{2}$ & $>280$ & & & $<140$ & $<70$ & & \\
\hline $\mathrm{SaO}_{2} / \mathrm{FiO}_{2}$ at & & $221-301$ & $142-220$ & $67-141$ & $<67$ & & \\
\hline $\begin{array}{l}\text { Coagulación } \\
\text { Plaquetas } \times 10^{3}\end{array}$ & $>150$ & $<150$ & $<100$ & $<50$ & $<20$ & & \\
\hline $\begin{array}{l}\text { Hepático } \\
\text { Bilirrubinas } \\
\mathrm{mg} / \mathrm{dl}\end{array}$ & $<1.2$ & $1.2-1.9$ & $2-5.9$ & $6-11.9$ & $>12$ & & \\
\hline $\begin{array}{l}\text { Cardiovascu- } \\
\text { lar }\end{array}$ & 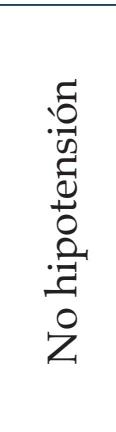 & 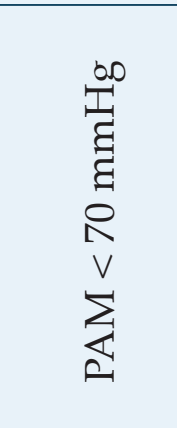 & 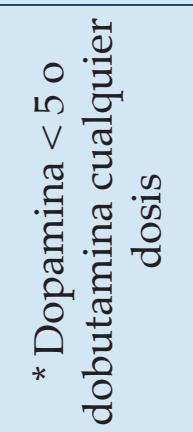 & 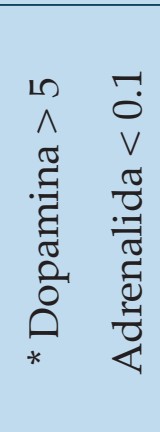 & 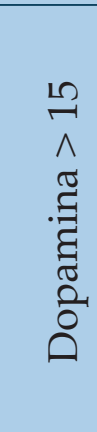 & 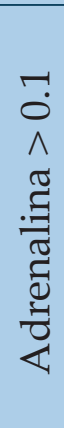 & 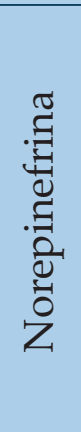 \\
\hline
\end{tabular}

* (microgramo) $/ \mathrm{Kg} /$ min durante al menos 1 hora 


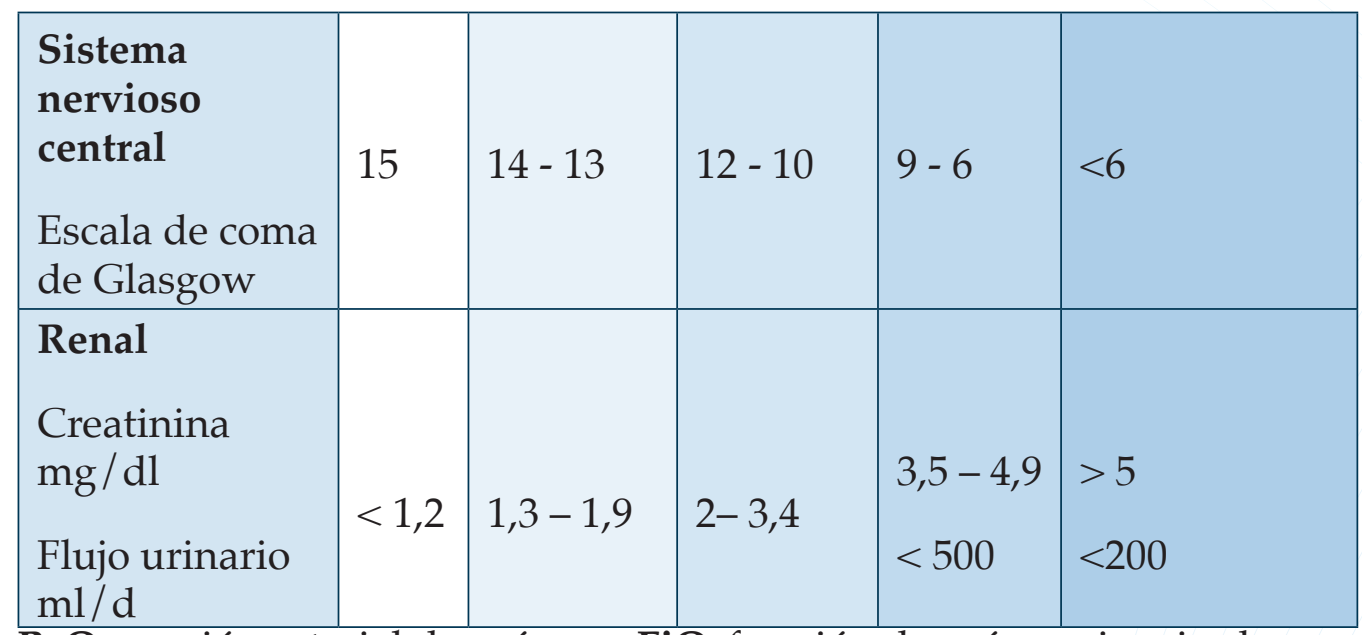

$\overline{\mathbf{P a O}}_{2:}$ presión arterial de oxígeno; $\mathbf{F i O}_{2}$ fracción de oxígeno inspirado;

$\mathrm{SaO}_{2}$ : saturación arterial de oxígeno periférico.

\section{Interpretación}

\begin{tabular}{|c|c|c|}
\hline \multirow{2}{*}{ PUNTUACIÓN } & $\begin{array}{c}\text { MORTALIDAD } \\
\text { EN CASO DE } \\
\text { PUNTUACIÓN } \\
\text { INICIAL }\end{array}$ & $\begin{array}{c}\text { MORTALIDAD EN EL } \\
\text { PUNTAJE MÁS ALTO }\end{array}$ \\
\hline $\mathbf{0 - 1}$ & $0 \%$ & $0 \%$ \\
\hline $\mathbf{2 - 3}$ & $6.4 \%$ & $1.5 \%$ \\
\hline $\mathbf{4 - 5}$ & $20.2 \%$ & $6.7 \%$ \\
\hline $\mathbf{6 - 7}$ & $21.5 \%$ & $18.2 \%$ \\
\hline $\mathbf{8 - 9}$ & $33.3 \%$ & $26.3 \%$ \\
\hline $\mathbf{1 0 - 1 1}$ & $50 \%$ & $45.8 \%$ \\
\hline $\mathbf{1 2 - 1 4}$ & $95.2 \%$ & $80 \%$ \\
\hline $\mathbf{> 1 4}$ & $95.2 \%$ & $89.7 \%$ \\
\hline
\end{tabular}

\section{Bibliografía}

\section{Original/Primaria}

Vincent JL, Moreno R, Takala J, et al. El puntaje SOFA (Evaluación de falla orgánica relacionada con sepsis) para describir la disfunción / falla orgánica. En nombre del Grupo de Trabajo sobre Problemas Relacionados 
con la Sepsis de la Sociedad Europea de Medicina Intensiva. Intensive Care Med [Internet]. 1996 Julio [citado marzo 2019] ;22(7):707-10. Disponible en: https: / / www.ncbi.nlm.nih.gov/ pubmed/8844239.

\section{Validación}

1. Vincent JL, de Mendonça A, Cantraine F, et al. Uso de la puntuación SOFA para evaluar la incidencia de disfunción / falla de órganos en unidades de cuidados intensivos: resultados de un estudio prospectivo multicéntrico. Grupo de trabajo sobre "problemas relacionados con la sepsis" de la Sociedad Europea de Medicina Intensiva. Crit Care Med [Internet]. 1998 [citado marzo 2019]; 26 (11): 1793-1800. Disponible en: https: / / www.ncbi.nlm.nih.gov/pubmed/9824069.

2. Ferreira FL, Bota DP, Bross A, Mélot C, Vincent JL. Evaluación en serie de la puntuación SOFA para predecir el resultado en pacientes críticamente enfermos. JAMA [Internet]. 2001 octubre [citadomarzo 2019]; 286 (14): 1754-8. Disponible en: https:// www.ncbi.nlm.nih. gov/pubmed/11594901.

3. Cárdenas-Turanzas M, Ensor J, Wakefield C, Zhang K, Wallace SK, Precio KJ. Validación cruzada de un modelo basado en la puntuación de la Evaluación de la Fase Orgánica Secuencial para predecir la mortalidad en pacientes con cáncer ingresados en la unidad de cuidados intensivos. J Crit Care [Internet]. Diciembre 2012 [citado marzo 2019]; 27 (6): 673-80. Disponible en: https:/ /www.ncbi.nlm. nih.gov/pubmed/22762932. 


\section{Q-SOFA (QUICK SOFA)}

\section{Utilidad}

Predice la mortalidad en la UCI, es una versión simplificada de SOFA.

\section{Descripción}

Úselo para predecir la mortalidad, no para diagnosticar la sepsis.

\section{Parámetros}

\begin{tabular}{|l|c|}
\hline CRITERIOS & PUNTOS \\
\hline Estado mental alterado GCS $<15$ & 1 \\
\hline Frecuencia respiratoria $\geq 22$ & 1 \\
\hline Presión arterial sistólica $\leq 100 \mathrm{mmHg}$ & 1 \\
\hline
\end{tabular}

GCS: Escala de coma de Glasgow

\section{Interpretación}

\begin{tabular}{|c|l|}
\hline PUNTUACIÓN & \multicolumn{1}{|c|}{ GRUPO DE RIESGO } \\
\hline $\mathbf{0 - 1}$ & No hay alto riesgo de mortalidad hospitalaria \\
\hline $\mathbf{2 - 3}$ & $\begin{array}{l}\text { Alto riesgo de mortalidad hospitalaria (aumento de } \\
\text { 3 a 14 veces) }\end{array}$ \\
\hline
\end{tabular}

\section{Bibliografía}

\section{Original/Primaria}

Seymour CW, Liu VX, Iwashyna TJ, et al.Evaluación de los criterios clínicos para la sepsis: para las definiciones del tercer consenso internacional para la sepsis y el shock séptico (sepsis-3). JAMA [Internet]. 2016 [citado marzo 2019]; 315 (8): 762-774. Disponible en: https: / /jamanetwork.com/ journals / jama/fullarticle/2492875. 


\section{Validación}

Vincent JL, de Mendonça A, Cantraine F, et al. Uso de la puntuación SOFA para evaluar la incidencia de disfunción / falla de órganos en unidades de cuidados intensivos: resultados de un estudio prospectivo multicéntrico. Grupo de trabajo sobre "problemas relacionados con la sepsis" de la Sociedad Europea de Medicina Intensiva. Crit Care Med [Internet]. 1998 [citado marzo 2019]; 26 (11): 1793-1800. Disponible en: https:/ / www. ncbi.nlm.nih.gov/pubmed/9824069.

Ferreira FL, Bota DP, Bross A, Mélot C, Vincent JL. Evaluación en serie de la puntuación SOFA para predecir el resultado en pacientes críticamente enfermos. JAMA [Internet]. 200110 octubre [citado marzo 2019]; 286 (14): 1754-8. Disponible en: https: / / www.ncbi.nlm.nih.gov/ pubmed/11594901.

Cárdenas-Turanzas M, Ensor J, Wakefield C, Zhang K, Wallace SK, Precio KJ. Validación cruzada de un modelo basado en la puntuación de la Evaluación de la Fase Orgánica Secuencial para predecir la mortalidad en pacientes con cáncer ingresados en la unidad de cuidados intensivos. J Crit Care [Internet]. Diciembre 2012 [citado marzo 2019]; 27 (6): 673-80. Disponible en: https:/ / www.ncbi.nlm.nih.gov/pubmed/22762932. 


\section{APACHE II}

\section{Utilidad}

Apache II es el acrónimo en inglés de Acute Physiology And Chronic Health Evaluation II, es un sistema de clasificación de severidad o gravedad de enfermedades, usado en las unidades de cuidados intensivos (UCI).

\section{Descripción}

- Apache II es la puntuación de predicción de mortalidad en la UCI más utilizada.

- Dado que Apache II se estudió en pacientes recién ingresados en la UCI, no es exacto cuando se trata de pacientes transferidos de otra unidad $\mathrm{u}$ hospital.

- Las puntuaciones de predicción de la UCI en general deben recalibrarse periódicamente para reflejar los cambios en la práctica y la demografía del paciente. 


\section{Parámetros}

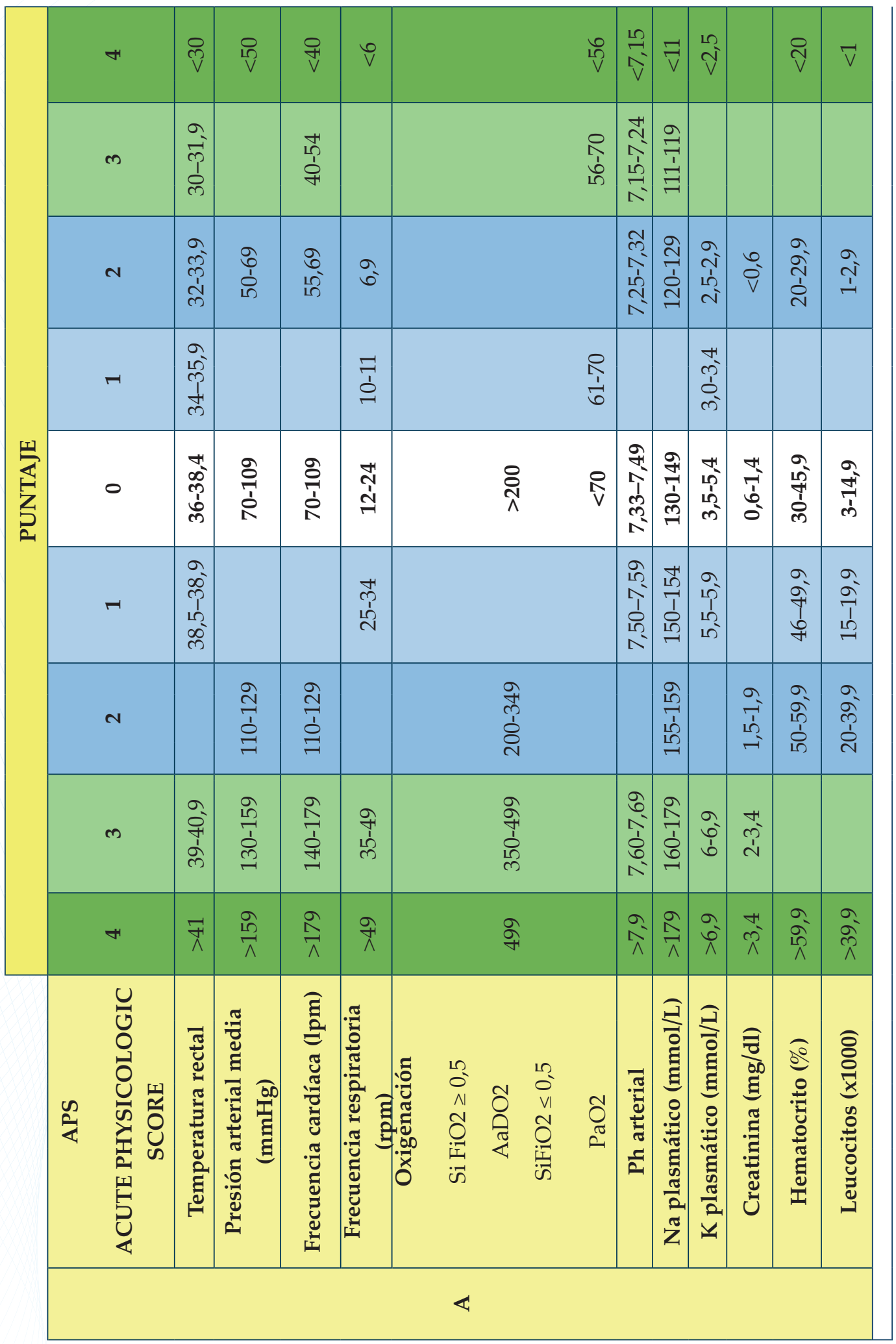




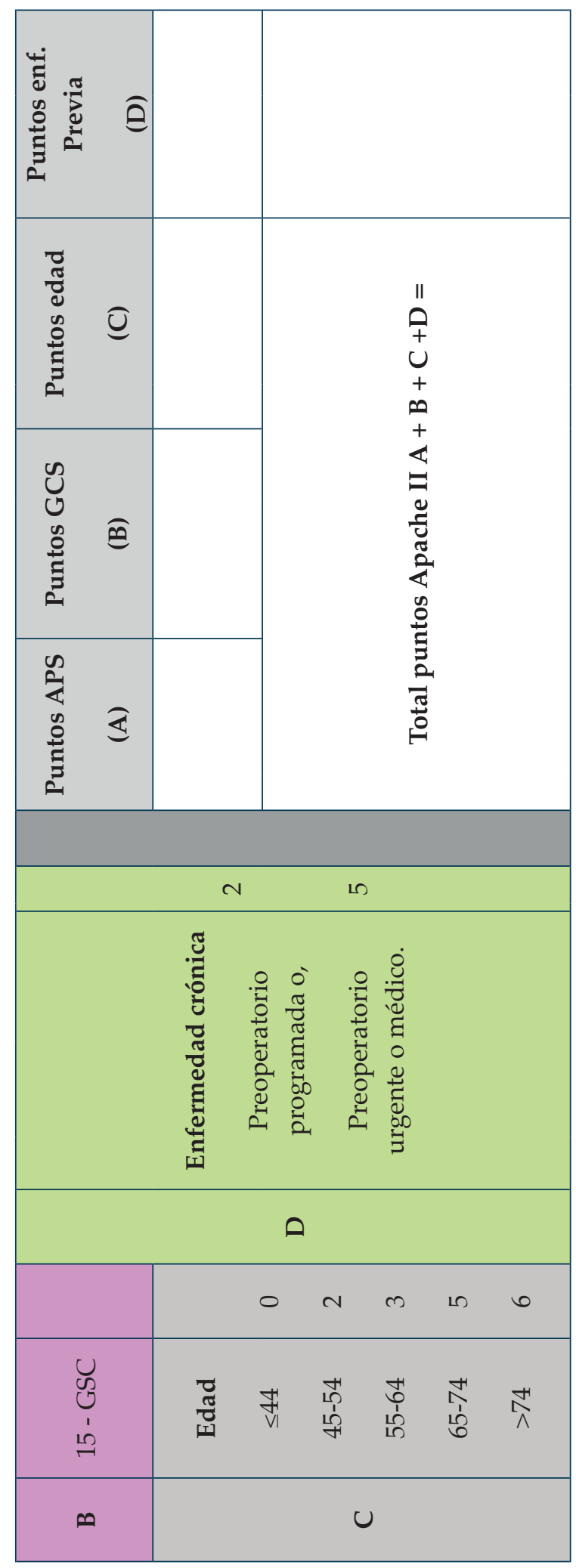




\section{Interpretación}

\begin{tabular}{|c|c|}
\hline PUNTUACIÓN & MORTALIDAD \\
\hline $\mathbf{0 - 4}$ & $4 \%$ \\
\hline $\mathbf{5 - 9}$ & $8 \%$ \\
\hline $\mathbf{1 0 - 1 4}$ & $15 \%$ \\
\hline $\mathbf{1 5 - 1 9}$ & $25 \%$ \\
\hline $\mathbf{2 0 - 2 4}$ & $40 \%$ \\
\hline $\mathbf{2 5 - 2 9}$ & $55 \%$ \\
\hline $\mathbf{3 0 - 3 4}$ & $73 \%$ \\
\hline$>34$ & $85 \%$ \\
\hline
\end{tabular}

\section{Bibliografía}

\section{Original/Primaria}

1. Knaus WA, Draper EA, Wagner DP, Zimmerman JE. APACHE II: un sistema de clasificación de la gravedad de la enfermedad. Crit Care Med [Internet]. Octubre 1985 [citado marzo 2019]; 13 (10): 818-29. Disponible en: https: / www.ncbi.nlm.nih.gov/pubmed/3928249.

\section{Validación}

1. Headley J, Theriault R, Smith TL. Validación independiente de la puntuación de gravedad de la enfermedad de APACHE II para predecir la mortalidad en pacientes con cáncer de mama ingresados en la unidad de cuidados intensivos. Cáncer [Internet]. 1992 julio [citado marzo 2019]; 70 (2): 497-503. Disponible en: https:/ / www.ncbi.nlm. nih.gov/pubmed/1617599.

2.

3. Capuzzo M, Valpondi V, Sgarbi A, Bortolazzi S, Pavoni V, Gilli G, Candini G, Gritti G, Alvisi R. Validación de los sistemas de puntuación de severidad SAPS II y APACHE II en una población de centro único. Intensive Care Med [Internet]. Diciembre 2000 [citado marzo 2019]; 26 (12): 1779-85. Disponible en: https://www.ncbi.nlm.nih.gov/ pubmed/11271085. 


\section{FARINGITIS ESTREPTOCÓCICA}

\section{PUNTAJE DE CENTOR (MODIFICADO / MCISAAC) PARA FARINGITIS ESTREPTOCÓCICA}

\section{Utilidad}

- Estima la probabilidad de que, la faringitis sea estreptocócica y sugiere un curso de manejo.

\section{Descripción}

- Utilizar solo en pacientes con faringitis aguda de inicio reciente $(\leq 3$ días).

\section{Parámetros}

\begin{tabular}{|l|l|c|}
\hline CRITERIO & CARACTERÍSTICAS & PUNTAJE \\
\hline Edad & $3-14$ & 1 \\
& $15-44$ & 0 \\
\hline $\begin{array}{l}\text { Exudado oños hipertrofia en las } \\
\text { amígdalas }\end{array}$ & No & -1 \\
\hline $\begin{array}{l}\text { Ganglios linfáticos cervicales } \\
\text { anteriores sensibles/ } \\
\text { inflamados }\end{array}$ & No & 0 \\
\hline Temperatura $>38^{\circ} \mathrm{C}$ & Sí & 1 \\
\hline Tos & No & 0 \\
\hline
\end{tabular}




\section{Interpretación}

\begin{tabular}{|c|c|l|}
\hline \multirow{2}{*}{ PUNTUACIÓN } & \multicolumn{1}{|c|}{$\begin{array}{l}\text { Probabilidad } \\
\text { de faringitis } \\
\text { estreptocócica }\end{array}$} \\
\hline $\mathbf{0}$ & $1-2.5 \%$ & $\begin{array}{l}\text { No hay más pruebas o } \\
\text { antibióticos. }\end{array}$ \\
\hline $\mathbf{1}$ & $5-10 \%$ & $\begin{array}{l}\text { Opcional prueba rápida de } \\
\text { estreptococos. }\end{array}$ \\
\hline $\mathbf{2}$ & $11-17 \%$ & $\begin{array}{l}\text { Considere pruebas rápidas de } \\
\text { estreptococos. }\end{array}$ \\
\hline $\mathbf{3}$ & $28-35 \%$ & $\begin{array}{l}\text { Considere pruebas rápidas de } \\
\text { estreptococos. Los antibióticos } \\
\text { empíricos pueden ser } \\
\text { apropiados dependiendo del } \\
\text { escenario específico. }\end{array}$ \\
\hline
\end{tabular}

\section{Bibliografía}

\section{Original/Primaria}

Centor RM, Witherspoon JM, Dalton HP, Brody CE, Link K. El diagnóstico de faringitis estreptocócica en adultos en la sala de emergencias. Making Med Decis [Internet]. 1981 [citado marzo 2019]; 1 (3): 239-46. Disponible en: https: / / www.ncbi.nlm.nih.gov/ pubmed/6763125.

\section{Validación}

McIsaac WJ, White D, Tannenbaum D, Low DE. Una puntuación clínica para reducir el uso innecesario de antibióticos en pacientes con dolor de garganta. CMAJ [Internet]. 1998 enero [citado marzo 2019]; 158 (1): $75-$ 83. Disponible en: https: / / www.ncbi.nlm.nih.gov/pubmed/ 9475915. 


\section{INFECCIONES DE TEJIDOS BLANDOS}

\section{PUNTUACIÓN LRINEC PARA LA INFECCIÓN NECROTIZANTE DE TEJIDOS BLANDOS}

\section{Utilidad}

La puntuación LRINEC (Indicador de riesgo de laboratorio para la fascitis necrotizante), es una escala que permite estratificar el riesgo de una persona con signos de celulitis para desarrollar fascitis necrotizante basándose en exámenes de laboratorio.

\section{Descripción}

Si existe una alta sospecha de fascitis necrotizante a través de la historia clínica y el examen físico, no calcule una puntuación LRINEC y vaya directamente al desbridamiento operatorio.

\section{Parámetros}

\begin{tabular}{|c|c|c|}
\hline CRITERIO & CARACTERÍSTICAS & PUNTAJE \\
\hline \multirow{2}{*}{ Proteína $\mathrm{C}$ reactiva } & $<15 \mathrm{mg} / \mathrm{dl}(150 \mathrm{mg} / \mathrm{l})$ & 0 \\
\hline & $\geq 15 \mathrm{mg} / \mathrm{dl}(150 \mathrm{mg} / \mathrm{l})$ & 4 \\
\hline \multirow{3}{*}{$\begin{array}{l}\text { Recuento de glóbulos blancos } \\
(\times 10,000 / \mu 1)\end{array}$} & $<15$ & 0 \\
\hline & $15-25$ & 1 \\
\hline & $>25$ & 2 \\
\hline \multirow{3}{*}{ Hemoglobina (g/dl) } & $>13.5$ & 0 \\
\hline & $11-13.5$ & 1 \\
\hline & $<11$ & 2 \\
\hline \multirow{2}{*}{ Sodio (mEq/1) } & $\geq 135$ & 0 \\
\hline & $<135$ & 2 \\
\hline \multirow{2}{*}{ Creatinina } & $\leq 1.6 \mathrm{mg} / \mathrm{dl}$ & 0 \\
\hline & $>1.6 \mathrm{mg} / \mathrm{dl}$ & 2 \\
\hline
\end{tabular}




\begin{tabular}{|l|l|l|}
\hline Glucosa & $\leq 180 \mathrm{mg} / \mathrm{dl}$ & 0 \\
& $>180 \mathrm{mg} / \mathrm{dl}$ & 1 \\
\hline
\end{tabular}

\section{Interpretación}

\begin{tabular}{|c|l|}
\hline PUNTUACIÓN & \multicolumn{1}{|c|}{ INTERPRETACIÓN } \\
\hline $\mathbf{2} 6$ & $\begin{array}{l}\text { Tiene una probabilidad importante para desarrollar } \\
\text { fascitis necrotizante. }\end{array}$ \\
\hline $\mathbf{<} 6$ & No descarta por completo el diagnóstico. \\
\hline
\end{tabular}

\section{Bibliografía}

\section{Original/Primaria}

1. Wong CH, Khin LW, Heng KS, Tan KC, bajo CO. La puntuación LRINEC (Indicador de riesgo de laboratorio para la fascitis necrotizante): una herramienta para distinguir la fascitis necrotizante de otras infecciones de tejidos blandos. Crit Care Med [Internet]. Julio 2004 [citado marzo 2019]; 32 (7): 1535-41. Disponible en: https: / / www.ncbi.nlm.nih.gov/ pubmed/15241098.

\section{Validación}

1. Chun-I Liao, Yi-Kung Lee, Yung-Cheng Su, Chin-Hsiang Chuang, Chun-Hing Wong. Validación del indicador de riesgo de laboratorio para la puntuación de fascitis necrotizante(LRINEC) para el diagnóstico temprano de la fascitis necrotizante. Tzu Chi Medical Journal [Internet]. junio 2012 [itado marzo 2019]; 24 (2):73-76. Disponible en: https:/ / www.sciencedirect.com/journal/tzu-chi-medical-journal. 


\section{Utilidad}

Predice la probabilidad de celulitis de las extremidades inferiores sobre otros diagnósticos.

\section{Descripción}

- Usar en pacientes adultos que acuden a la sala de urgencias, y en quienes se tiene sospecha clínica de celulitis.

- No usar en:

- Absceso / fluctuación visible.

- Trauma penetrante.

- Quemadura.

- Úlcera diabética.

- Hardware / dispositivo implantado.

- Paciente postoperatorio.

- Uso reciente de antibióticos por vía intravenosa (dentro de las 48 horas).

\section{Parámetros}

\begin{tabular}{|c|l|c|}
\hline \multicolumn{2}{|c|}{ CRITERIO } & PUNTAJE \\
\hline $\mathbf{A}$ & Asimétrico & 3 \\
\hline $\mathbf{L}$ & Leucocitosis $(\geq 10,000 / \mu \mathrm{L})$ & 1 \\
\hline $\mathbf{T}$ & Taquicardia & 1 \\
\hline $\mathbf{7 0}$ & Edad $\geq 70$ & 2 \\
\hline
\end{tabular}

\section{Interpretación}

\begin{tabular}{|c|c|}
\hline PUNTUACIÓN & INTERPRETACIÓN/RECOMENDACIÓN \\
\hline $0-2$ & $\begin{array}{l}\text { Reevaluar ( }>83.3 \% \text { de probabilidad de } \\
\text { pseudocelulitis). }\end{array}$ \\
\hline $3-4$ & Consultar dermatología y / o infectología \\
\hline $5-7$ & $\begin{array}{l}\text { Tratamiento ( }>82.2 \% \text { de probabilidad de celulitis } \\
\text { verdadera). }\end{array}$ \\
\hline
\end{tabular}




\section{Bibliografía}

\section{Original/Primaria}

1. Raff AB, Weng QY, Cohen JM, y col. Un modelo predictivo para el diagnóstico de la celulitis de las extremidades inferiores: un estudio transversal. J Am Acad Dermatol [Internet]. 2017 [citado marzo 2019]; 76 (4): 618-625. Disponible en: https:/ / www.jaad.org/article/S01909622(17)30018-X/abstract.

\section{Validación}

1. Li DG, Dewan AK, Di Xia F, Khosravi H, Joyce C, Mostaghimi A. El modelo predictivo ALT-70 supera la imagen térmica para el diagnóstico de la celulitis de las extremidades inferiores: una evaluación prospectiva. J Am Acad Dermatol [Internet]. 2018 [citado marzo 2019]; 79(6): 1076-1080. Disponible en: https://www.sciencedirect.com/ science/ article/ abs / pii/S0190962218322217.

2. Singer S, Li DG, Gunasekera N, Okhovat JP, Vedak P. El modelo predictivo ALT-70 mantiene el valor predictivo a las 24 y 48 horas después de la presentación. J Am Acad Dermatol [Internet]. 2019 [citado marzo 2019]. S0190-9622 (19) 30468-2. Disponible en: https:/ / www.ncbi.nlm.nih.gov/pubmed/30914341. 


\section{FIEBRE REUMÁTICA}

\section{CRITERIOS DE JONES PARA DIAGNÓSTICO DE FIEBRE REUMÁTICA}

\section{Utilidad}

Diagnostica la fiebre reumática aguda en función de criterios mayores y menores.

\section{Descripción}

- Usar en ataques iniciales de fiebre reumática aguda.

- No utilice para medir la actividad reumática, establecer el diagnóstico de enfermedad cardíaca reumática inactiva o crónica, o predecir el curso o la gravedad de la enfermedad.

\section{Parámetros}

\section{CRITERIOS REQUERIDOS}

\section{Cultivo de garganta positivo o prueba rápida de estreptococos}

Título de anticuerpos estreptocócicos elevado o en aumento

\section{CRITERIOS MAYORES}

Carditis
Poliartritis
Corea
Eritema marginado
Nódulos subcutáneos

\begin{tabular}{|l|}
\hline CRITERIOS MENORES \\
\hline Frtralgia \\
Fiebre \\
Reactivos de fase aguda elevedos \\
Velocidad de sedimentación globular \\
Proteína C reactiva \\
Intervalo PR prolongado \\
\hline
\end{tabular}




\section{Interpretación}

\section{INTERPRETACIÓN}

DIAGNÓSTICO POSITIVO
1 criterio requerido

2 criterios mayores

1 criterio mayor +2 criterios menores

\section{Bibliografía}

\section{Original/Primaria}

1. Dajani AS, Ayoub E, Bierman FZ. Pautas para el diagnóstico de la fiebre reumática. Criterios de Jones, actualización de 1992. JAMA [Internet]. 1992 [citado marzo 2019]; 268 (15): 2069-73. Disponible en: https:// jamanetwork.com/journals/ jama/article-abstract/ 400639.

\section{Referencias}

1. Gewitz MH, Baltimore RS, Tani LY, et al. Revisión de los Criterios de Jones para el diagnóstico de la fiebre reumática aguda en la era de la ecocardiografía Doppler: una declaración científica de la American Heart Association. Circulation [Internet]. 2015 [citado marzo 2019]; 131 (20): 1806-18. Disponible en: https:/ / www.ahajournals.org/doi / full/10.1161/ cir.0000000000000205. 


\section{VIH}

\section{PUNTUACIÓN DE RIESGO DE DENVER}

\section{Utilidad}

Predice la probabilidad de infección por VIH no diagnosticada.

\section{Parámetros}

\begin{tabular}{|c|c|c|}
\hline CRITERIO & CARACTERÍSTICAS & PUNTAJE \\
\hline \multirow{7}{*}{ Edad } & $<22$ & 0 \\
\hline & $22-25$ & 4 \\
\hline & $26-32$ & 10 \\
\hline & $33-46$ & 12 \\
\hline & $47-54$ & 10 \\
\hline & $55-60$ & 4 \\
\hline & $>60$ & 0 \\
\hline \multirow{2}{*}{ Sexo } & Femenino & 0 \\
\hline & Masculino & 21 \\
\hline \multirow{5}{*}{ Raza } & Negro & 0 \\
\hline & Hispano & \\
\hline & Indio americano, nativo de Alaska, & \\
\hline & $\begin{array}{l}\text { hawaiano nativo, isleño del Pacífico } \\
\text { no hawaiano u otro. }\end{array}$ & 0 \\
\hline & & 0 \\
\hline \multicolumn{3}{|l|}{ Prácticas sexuales } \\
\hline \multirow{4}{*}{$\begin{array}{l}\text { Elija la práctica que } \\
\text { obtenga el puntaje más } \\
\text { alto, por ejemplo, si el } \\
\text { paciente se involucra } \\
\text { en relaciones sexuales } \\
\text { anales tanto vaginales } \\
\text { como receptivas, elija } \\
\text { esta última para asignar } \\
8 \text { puntos. }\end{array}$} & Sexo con un hombre & 22 \\
\hline & Coito anal receptivo & 8 \\
\hline & Coito vaginal & -10 \\
\hline & Ninguna de las anteriores & 0 \\
\hline
\end{tabular}




\begin{tabular}{|l|l|c|}
\hline \multirow{2}{*}{ Otros factores de riesgo } & Uso de drogas inyectables & 9 \\
& Pruebas de VIH en el pasado & -4 \\
& Ninguno & 0 \\
\hline
\end{tabular}

Interpretación

\begin{tabular}{|c|l|}
\hline PUNTUACIÓN & \multicolumn{1}{c|}{ INTERPRETACIÓN } \\
\hline$<20$ & Muy bajo \\
\hline $20-29$ & Bajo \\
\hline $30-39$ & Moderado \\
\hline $40-49$ & Alto \\
\hline$\geq 50$ & Muy alto \\
\hline
\end{tabular}

\section{Bibliografía}

\section{Original/Primaria}

1. Haukoos JS, Lyons MS, Lindsell CJ, et al. Obtención y validación de la puntuación de riesgo del Virus de Inmunodeficiencia Humana (VIH) de Denver para la detección del VIH dirigida. Am J Epidemiol [Internet]. 2012 [citado marzo 2019]; 175 (8): 838-46. Disponible en https: / / academic.oup.com/aje/article/175/8/838/202955.

\section{Validación}

1. Haukoos JS, Hopkins E, Bender B, et al. Comparación de la detección mejorada y dirigida rápida del VIH utilizando la puntuación de riesgo de VIH de Denver con la detección rápida no dirigida del VIH en el departamento de emergencias Ann Emerg Med [Internet]. 2013 [citado marzo 2019]; 61 (3): 353-61. Disponible en: https://www. annemergmed.com/article/S0196-0644(12)01704-0/abstract. 


\section{NEUTROPENIA FEBRIL}

\section{ÍNDICE CLÍNICO DE NEUTROPENIA FEBRIL ESTABLE (CISNE)}

\section{Utilidad}

El Clinical Index of Stable Febrile Neutropenia (CISNE), identifica complicaciones en pacientes con neutropenia febril aparentemente estable.

\section{Descripción}

Usar en:

- Pacientes ambulatorios adultos, mayores de 18 años de edad con tumor sólido, fiebre de al menos $38^{\circ} \mathrm{C}$ durante 1 hora y neutropenia.

- No usar en:

- Insuficiencia orgánica aguda (renal, cardíaca y respiratoria).

- Descompensación de insuficiencia crónica de órganos.

- Choque séptico e hipotensión (PA sistólica $<90$ mmHg).

- Infección severa conocida.

- Otras complicaciones graves que a su vez requerirían ingreso.

\section{Parámetros}

\begin{tabular}{|l|c|c|}
\hline CRITERIO & CARACTERÍSTICAS & PUNTAJE \\
\hline Estado de rendimiento de ECOG & $<2$ & 0 \\
$\begin{array}{l}\text { ECOG PS 2 = Capaz de todos } \\
\text { los cuidados personales, pero no } \\
\text { actividades de trabajo, fuera de la } \\
\text { cama }>50 \% \text { del día. }\end{array}$ & $\geq 2$ & 2 \\
\hline $\begin{array}{l}\text { Hiperglucemia inducida por estrés } \\
\text { Nivel de glucosa en sangre inicial } \geq 121 \\
\text { mg/dL, o } \geq 250 \text { mg/dL en diabéticos o } \\
\text { si utilizan esteroides. }\end{array}$ & No & 0 \\
\hline
\end{tabular}




\begin{tabular}{|l|c|c|}
\hline EPOC & No & 0 \\
$\begin{array}{l}\text { Diagnóstico de EPOC en terapia } \\
\text { con } \geq 1 \text { de los siguientes: esteroides, } \\
\text { suplementos de O2, broncodilatadores. }\end{array}$ & Sí & 1 \\
\hline $\begin{array}{l}\text { Historia de enfermedad cardiovascular } \\
\text { Afecciones cardíacas crónica, } \\
\text { excluyendo la historia de AFib. }\end{array}$ & No & 0 \\
\hline $\begin{array}{l}\text { Grado de mucositis NCI } \geq 2 \quad \text { No } \\
\begin{array}{l}\text { Eritema, edema o úlceras dolorosos, } \\
\text { pero es posible comer / tragar. }\end{array}\end{array}$ & SÍ & 0 \\
\hline Monocitos & $\geq 200 / \mu 1$ & 0 \\
\hline
\end{tabular}

ECOG: eastern cooperative oncology group; EPOC: enfermedad pulmonar obstructiva crónica;

NCI: national cancer institute.

\section{Interpretación}

\begin{tabular}{|c|c|c|c|}
\hline PUNTAJE & $\begin{array}{l}\text { CATEGORÍA } \\
\text { DE RIESGO }\end{array}$ & $\begin{array}{c}\text { RIESGO DE } \\
\text { COMPLICACIONES }\end{array}$ & RECOMENDACIONES \\
\hline 0 & $\begin{array}{c}\text { I } \\
\text { (bajo) }\end{array}$ & $1.1 \%$ & $\begin{array}{l}\text { Considere el alta } \\
\text { hospitalaria con } \\
\text { antibióticos orales } \\
\text { después de una } \\
\text { discusión con } \\
\text { oncología. }\end{array}$ \\
\hline $1-2$ & $\begin{array}{c}\text { II } \\
\text { (Intermedio) }\end{array}$ & $6.2 \%$ & $\begin{array}{l}\text { Utilice el juicio clínico } \\
\text { con respecto a la } \\
\text { admisión. Considere la } \\
\text { consulta oncológica. }\end{array}$ \\
\hline$\geq 3$ & $\begin{array}{c}\text { III } \\
\text { (alto) }\end{array}$ & $36 \%$ & $\begin{array}{l}\text { Admitir para mayor } \\
\text { investigación, } \\
\text { incluyendo } \\
\text { hemocultivos. }\end{array}$ \\
\hline
\end{tabular}




\section{Bibliografía}

\section{Original/Primaria}

1. Carmona-Bayonas A, Gómez J, González-billalabeitia E, et al. Evaluación pronostica de la neutropenia febril en pacientes adultos con cáncer aparentemente estables. Br J Cancer [Internet]. 2011 [citado marzo 2019]; 105 (5): 612. Disponible en: https:/ / www.ncbi.nlm.nih. gov/pubmed / 21811253 .

\section{Validación}

1. Carmona-Bayonas A, Jiménez-fonseca P, Virizuela echaburu J, et al. Predicción de complicaciones graves en pacientes con neutropenia febril aparentemente estable: validación del índice clínico de neutropenia febril estable en una cohorte prospectiva de pacientes del estudio FINITE. J Clin Oncol. [Internet] 2015 [citado marzo 2019]; 33 (5): 465-71. Disponible en: https: / / www.ncbi.nlm.nih.gov / pubmed / 25559804 .

2. Coyne CJ, Le V, Brennan JJ, Castillo EM, Shatsky RA, et al. Aplicación de los puntajes de estratificación de riesgo de MASCC y CISNE para identificar pacientes con neutropileno febril de bajo riesgo en el servicio de urgencias. Ann Emerg Med [Internet]. 2017 [citado marzo 2019]. Disponible en: https: / / www.ncbi.nlm.nih.gov/pubmed/28041827.

3. Flores CR, Seidenfeld J, Bow EJ, et al. La profilaxis antimicrobiana y el tratamiento ambulatorio de la fiebre y la neutropenia en adultos tratados por enfermedades malignas: guía de práctica clínica de la American Society of Clinical Oncology. J Clin Oncol [Internet]. 2013 [citado marzo 2019]; 31 (6): 794-810. Disponible en: https:/ / ascopubs. org/doi / full/10.1200/jco.2012.45.8661.

4. Fonseca PJ, Carmona-bayonas A, García IM, et al. Un nomograma para la predicción de complicaciones en pacientes con tumores sólidos y neutropenia febril aparentemente estable. Br J Cancer [Internet]. 2016 [citado marzo 2019]; 114 (11): 1191- Disponible en: https: / / www.ncbi. nlm.nih.gov/pmc/articles/PMC4891503/. 


\section{ÍNDICE DE RIESGO MASSCC}

\section{Utilidad}

El índice MASSCC (Multinational Association for Supportive Cancer in Care), permite evaluar el riesgo de complicaciones y la mortalidad esperada en pacientes con neoplasias, que presentan neutropenia febril.

\section{Descripción}

- Usar en pacientes adultos con neutropenia, con fiebre de al menos 38 ${ }^{\circ} \mathrm{C}$.

- No usar en pacientes con leucemia aguda, sometidos a quimioterapia de inducción o acondicionamiento alogénico de trasplante de células madre hematopoyéticas, según las pautas de IDSA (Sociedad Americana de Enfermedades Infecciosas).

\section{Parámetros}

\begin{tabular}{|c|c|c|}
\hline CRITERIO & CARACTERÍSTICAS & PUNTAJE \\
\hline $\begin{array}{l}\text { Carga de la enfermedad } \\
\text { (gravedad de los síntomas) }\end{array}$ & Ninguno o leve & 5 \\
\hline \multirow{2}{*}{$\begin{array}{l}\text { Según lo determinado por } \\
\text { el médico de cabecera en la } \\
\text { presentación. }\end{array}$} & Moderado & 3 \\
\hline & Severo & 0 \\
\hline Hipotensión & No & 5 \\
\hline Presión sistólica < 90mmHg. & Sí & 0 \\
\hline \multirow{3}{*}{$\begin{array}{l}\text { EPOC activa } \\
\text { Bronquitis crónica activa, } \\
\text { enfisema, FEV disminuido o } \\
\text { necesidad de oxigenoterapia, } \\
\text { corticosteroides } \\
\text { broncodilatadores. }\end{array}$} & & \\
\hline & No & 4 \\
\hline & Sí & 0 \\
\hline
\end{tabular}




\begin{tabular}{|l|l|l|}
\hline & $\begin{array}{l}\text { Tumor sólido } \\
\text { Hematológica, sin } \\
\text { infección micótica previa. } \\
\text { Hematológica, infección } \\
\text { micótica previa. }\end{array}$ & 4 \\
\hline $\begin{array}{l}\text { Deshidratación que } \\
\text { requiere líquidos por vía } \\
\text { intravenosa. }\end{array}$ & No & 3 \\
\hline Sstado al inicio de la fiebre & Paciente externo & 0 \\
\hline Paciente interno & 3 \\
\hline Edad & $<60$ & 0 \\
\hline
\end{tabular}

FEV: volumen espiratorio forzado.

\section{Interpretación}

\begin{tabular}{|c|l|l|}
\hline PUNTAJE & $\begin{array}{c}\text { CATEGORÍA } \\
\text { DE RIESGO }\end{array}$ & \multicolumn{1}{|c|}{ RECOMENDACIONES } \\
\hline$<\mathbf{2 1}$ & Riesgo bajo & $\begin{array}{l}\text { Considere la posibilidad de terapia } \\
\text { antibiótica empírica oral y/o ambulatoria. }\end{array}$ \\
\hline $\mathbf{2 1}$ & Alto riesgo & $\begin{array}{l}\text { Utilice antibióticos intravenosos de forma } \\
\text { empírica. Requiere ingreso hospitalario. }\end{array}$ \\
\hline
\end{tabular}

\section{Bibliografía}

\section{Original/Primaria}

1. Klastersky J, Paesmans M, Rubenstein EB, Boyer M, Elting L, Feld R, Gallagher J, Herrstedt J, Rapoport B, Rolston K, Talcott J. La Asociación Multinacional de Atención en el Índice de Riesgo de Cáncer: un sistema de puntuación multinacional para Identificación de pacientes con cáncer neutropénico febril de bajo riesgo. J Clin Oncol [Internet]. 2002 agosto [citado marzo 2019]; 18 (16): 3038-51. Disponible en: https:// www.ncbi.nlm.nih.gov/pubmed/10944139. 


\section{Validación}

1. Uys A, Rapoport BL, Anderson R. Neutropenia febril: un estudio prospectivo para validar la puntuación del índice de riesgo de la Asociación Multinacional de Cuidados de Apoyo para el Cáncer (MASCC). Atención de apoyo para el cáncer [Internet]. 2004 [citado marzo 2019]; 12 (8): 555-60. Disponible en: https://www.ncbi.nlm. nih.gov/pubmed/15197637.

2. Klastersky J, Paesmans M, Georgala A, Muanza F, Plehiers B, et al. Antibióticos orales ambulatorios para pacientes con cáncer neutropénico febril que utilizan una puntuación predictiva de complicaciones. J Clin Oncol [Internet]. 2006 [citado marzo 2019]; 24 (25): 4129-34. Disponible en: https://www.ncbi.nlm.nih.gov/ pubmed / 16943529 .

3. Cherif $\mathrm{H}$, Johansson E, Björkholm M, Kalin M. La viabilidad del alta hospitalaria temprana con terapia antimicrobiana oral en pacientes de bajo riesgo con neutropenia febril después de la quimioterapia para enfermedades malignas hematológicas. Haematologica [Internet]. 2006 [citaod marzo 2019]; 91(2):215-22. Disponible en: https:/ / www. ncbi.nlm.nih.gov/pubmed/16461306.

4. Innes H, Lim SL, Sala A, Chan SY, Bhalla N, Marshall E. Manejo de la neutropenia febril en tumores sólidos y linfomas mediante el índice de riesgo de la Asociación Multinacional de Atención de Apoyo en Cáncer (MASCC): viabilidad y seguridad en la práctica clínica de rutina. Atención de apoyo para el cáncer [Internet]. 2008 [citaod marzo 2019] ; 16 (5): 485-91. Disponible en: https: / / www.ncbi.nlm.nih.gov / pubmed/17899215.

5. Baskaran ND, Gan GG, Adeeba K. Aplicación de la Asociación Multinacional para la Atención de Apoyo en la puntuación del riesgo de cáncer al predecir el resultado de los pacientes con neutropenia febril en una cohorte de pacientes. Ann Hematol [Internet]. 2008 [citado marzo 2019];87(7):563-9. Disponible en: https://www.ncbi. nlm.nih.gov/pubmed/18437382.

6. Carmona-Bayonas A, Gómez J, González-billalabeitia E, et al. Evaluación pronóstica de la neutropenia febril en pacientes adultos con cáncer aparentemente estables. Br J Cancer [Internet]. 2011 [citado marzo 2019]; 105 (5): 612. Disponible en: https:/ / www.ncbi.nlm.nih. gov/pubmed / 21811253 .

7. Carmona-Bayonas A, Jiménez-fonseca P, Virizuela echaburu J, et al. Predicción de complicaciones graves en pacientes con neutropenia febril aparentemente estable: validación del índice clínico de neutropenia febril estable en una cohorte prospectiva de pacientes 
del estudio FINITE. J Clin Oncol. [Internet] 2015 [citado marzo 2019]; 33 (5): 465-71. Disponible en: https:/ / www.ncbi.nlm.nih.gov/ pubmed / 25559804 .

8. Coyne CJ, Le V, Brennan JJ, Castillo EM, Shatsky RA, et al. Aplicación de los puntajes de estratificación de riesgo de MASCC y CISNE para identificar pacientes con neutropileno febril de bajo riesgo en el servicio de urgencias. Ann Emerg Med [Internet]. 2017 [citado marzo 2019]. Disponible en: https: / / www.ncbi.nlm.nih.gov/pubmed/28041827. 
CÁCULOSY

PROHÓSTICAS

EN MEDICINA INTERNA 


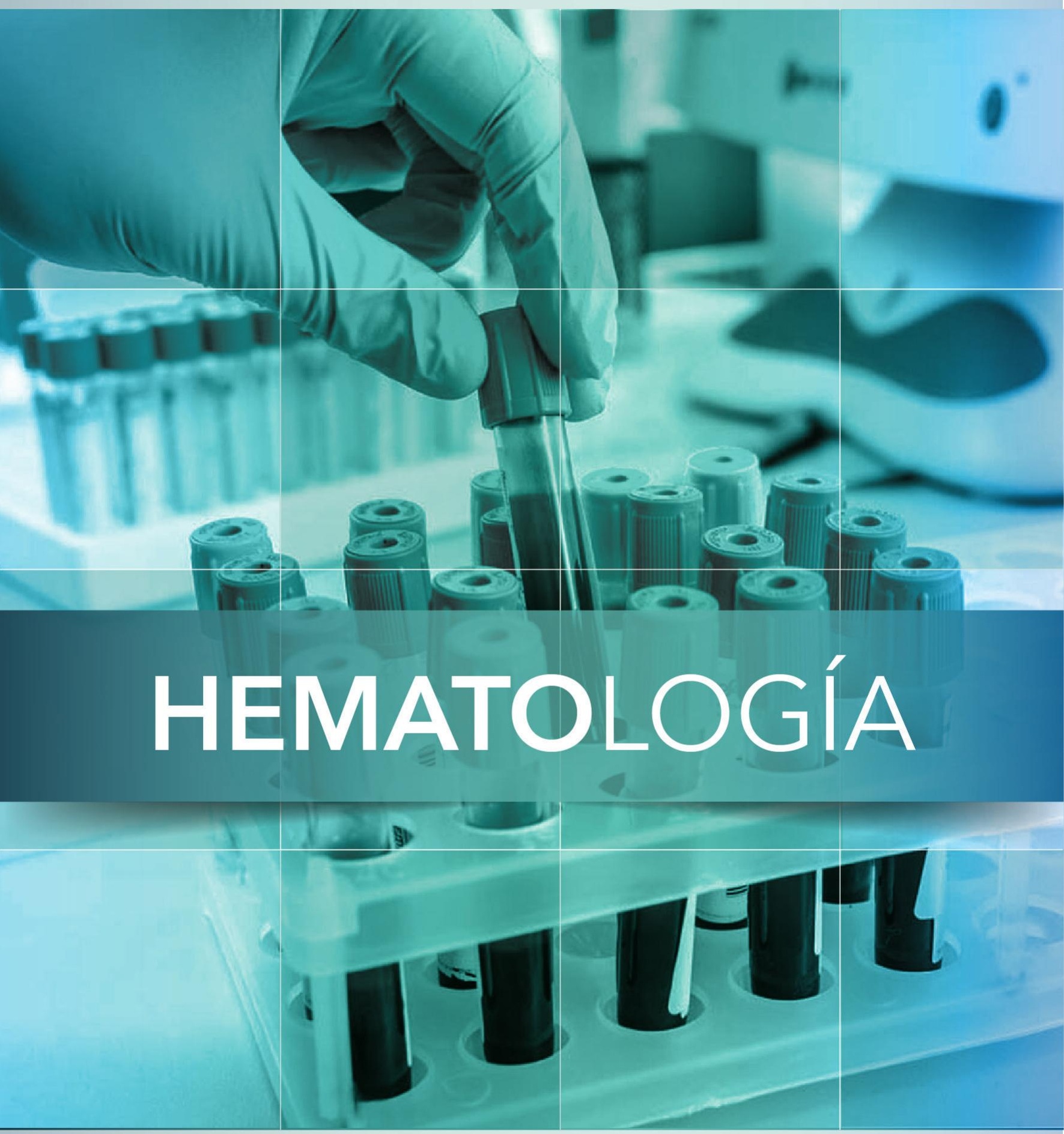




\section{RECUENTO ABSOLUTO DE LINFOCITOS (ALC)}

\section{Utilidad}

Evalúa el número de linfocitos y predice el recuento de CD4.

\section{Descripción}

- Normalmente, esto se usa en países del tercer mundo donde las pruebas de CD4 son caras o no están disponibles.

\section{Parámetros}

Linfocitos

Recuento de glóbulos blancos
$\%$

de 3,7 a $10,7 \times 10^{3}$ células / uL

\section{Fórmula}

$A L C=($ Recuento de glóbulos blancos $x$ 1000) $x \%$ de linfocitos

\section{Interpretación}

\begin{tabular}{|l|l|}
\hline \multicolumn{1}{|c|}{ ALC } & \multicolumn{1}{|c|}{ Recuento de CD4 predicho } \\
\hline$<\mathbf{1 0 0 0} \mathrm{cel} / \mathbf{~ m m}^{3}$ & $<200$ células $/ \mathrm{mm}^{3}$ \\
\hline $\mathbf{1 0 0 0}-\mathbf{2 0 0 0}$ celdas $/ \mathbf{~ m m}^{3}$ & Incapaz de predecir \\
\hline$>\mathbf{2 0 0 0}$ celulas $/ \mathbf{m m ~ 3}$ & $>200$ células $/ \mathrm{mm}^{3}$ \\
\hline
\end{tabular}

\section{Bibliografía}

\section{Original}

1. Shapiro NI, Karras DJ, Leech SH, Heilpern KL. Recuento absoluto de linfocitos como predictor del recuento de CD4. Ann Emerg Med [Internet]. Septiembre 1998 [citado marzo 2019]; 32 (3 Pt 1): 323-8. Disponible en: https: / / www.ncbi.nlm.nih.gov/pubmed/9737494. 


\section{RECUENTO ABSOLUTO DE NEUTRÓFILOS (ANC)}

\section{Utilidad}

Con frecuencia se utiliza para evaluar la fiebre neutropénica en pacientes con quimioterapia.

\section{Descripción}

1. El ANC puede ser crítico para evaluar el riesgo de un paciente inmunocomprometido de desarrollar infecciones oportunistas.

2. El cálculo de ANC, no es una medida estática que se realiza solo una vez al ingreso en el hospital; a menudo se mide diariamente en pacientes críticos para evaluar la respuesta de la médula ósea después de la quimioterapia, por ejemplo.

\section{Parámetros}

Neutrófilos

Bandas

Recuento de glóbulos blancos de 3,7 a 10,7 x 10³ células / uL

Fórmula

$A N C=10 X$ Recuento de glóbulos blancos $x$ (\% Neutrófilos+bandas)

\section{Interpretación}

\begin{tabular}{|l|l|}
\hline \multicolumn{1}{|c|}{ ANC } & \multicolumn{1}{c|}{ INTERPRETACIÓN } \\
\hline $\mathbf{1 . 0 0 0 - 1 . 5 0 0}$ células $/ \mathrm{mm}^{3}$ & Neutropenia leve \\
\hline $\mathbf{5 0 0 - 9 9 9}$ células $/ \mathrm{mm}^{3}$ & Neutropenia moderada \\
\hline$<500$ células $/ \mathrm{mm}^{3}$ & Neutropenia grave \\
\hline
\end{tabular}

\section{Bibliografía}

\section{Original}

1. Al-Gwaiz LA, Babay HH. El valor diagnóstico del recuento absoluto de neutrófilos, recuento de bandas y cambios morfológicos de los 
neutrófilos en la predicción de infecciones bacterianas. Med Princ Pract [Internet]. 2007 [citado marzo 2019]; 16 (5): 344-7. Disponible en: https: / / www.ncbi.nlm.nih.gov/pubmed/17709921.

\section{Validación}

1. Thomas BN, Karen MP, Soora W, David D, Gabriel JE. Interpretación de los recuentos sanguíneos completos poco después del nacimiento en recién nacidos con riesgo de septicemia. Pediatrics [Internet]. 2010 [citado marzo 2019]; 126 (5): 903-909. Disponible en: https://www. ncbi.nlm.nih.gov/pmc/articles/PMC3197862/. 


\section{ÍNDICE ABSOLUTO DE RETICULOCITOS E ÍNDICE DE RETICULOCITOS}

\section{Utilidad}

Evalúa la respuesta apropiada a la anemia, frecuentemente en pacientes con células falciformes.

\section{Descripción}

- El recuento de reticulocitos de laboratorio es en realidad un porcentaje. El recuento absoluto corrige el nivel de anemia, y el índice de reticulocitos determina si el recuento de reticulocitos es apropiado para el nivel de anemia.

\section{Parámetros}

Reticulocitos

Hematocrito

$\%$

Recuento de glóbulos blancos de 3,7 a 10,7 x 103 células / uL

\section{Fórmula}

Recuento absoluto de reticulocitos $=\%$ de reticulocitos $x\left(\frac{\text { hcto paciente }}{\text { hcto normal }}\right)$ indice de reticulocitos $=\frac{\text { recuento absoluto de reticulocitos }}{\text { factor de maduración }}$

\begin{tabular}{|c|c|}
\hline HEMATOCRITO & FACTOR DE MADURACIÓN \\
\hline $\mathbf{2 3 5 \%}$ & 1.0 \\
\hline $\mathbf{2 5} \mathrm{a}<35 \%$ & 1.5 \\
\hline $\mathbf{2 0} \mathrm{a}<\mathbf{2 5 \%}$ & 2.0 \\
\hline$<\mathbf{2 0} \%$ & 2.5 \\
\hline
\end{tabular}




\section{Interpretación}

\section{ÍNDICE DE RETICULOCITOS INTERPRETACIÓN}

\begin{tabular}{|l|l|}
\hline$\geq 2$ & Respuestaadecuada(arregenerativa) \\
\hline$<2$ & Hipoproliferación (regenerativa) \\
\hline
\end{tabular}

\section{Bibliografía}

\section{Original}

1. Priya PP, ARS Papel del recuento absoluto de reticulocitos en la evaluación de un estudio de pancitopenia-A en un hospital. Revista de investigación clínica y de diagnóstico: J Clin Diagn Res [Internet] 2014 agosto [citado marzo 2019]; 8 (8): FC01-FC03. Disponible En: https: / / www.ncbi.nlm.nih.gov/pmc/articles/PMC4190718/\#_ffn_sectitle. 


\section{ECUACIÓN DE GANZONI PARA LA ANEMIA POR DEFICIENCIA DE HIERRO}

\section{Utilidad}

Calcula el déficit de hierro para la dosificación de hierro.

\section{Descripción}

Usar en pacientes con anemia por deficiencia de hierro, en quienes se considera la suplementación con hierro.

\section{Parámetros}

Peso

Hemoglobina objetivo

$\mathrm{kg}$

Hemoglobina real

Reservas de hierro

\section{Fórmula}

$$
\begin{aligned}
& \mathrm{g} / \mathrm{dl} \\
& \mathrm{g} / \mathrm{dl} \\
& \mathrm{mg} \\
& \text { - Usar } 500 \mathrm{mg} \text { para adultos y niños } \geq 35 \mathrm{~kg} \\
& \text { - } 15 \mathrm{mg} / \mathrm{kg} \text { en niños }<35 \mathrm{~kg}
\end{aligned}
$$

Déficit total de hierro=Peso $(\mathrm{kg}) x(\mathrm{HbObjetivo}(\mathrm{g} / \mathrm{dl})-\mathrm{Hbreal}(\mathrm{g} / \mathrm{dl}) x$ $2.4+$ reservas de hierro $(m g)$.

\section{Bibliografía}

\section{Original}

1. Ganzoni AM.[Hierro-dextrano intravenoso: posibilidades terapéuticas y experimentales]. Schweiz Med Wochenschr [Internet]. 1970 [citado marzo 2019]; 100 (7): 301-3. Disponible en: https:/ / www.ncbi.nlm. nih.gov/pubmed/5413918/.

\section{Validación}

1. Koch TA, Myers J, Goodnough LT. Tratamiento con hierro por vía intravenosa en pacientes con anemia por deficiencia de hierro: Consideraciones sobre la dosificación. Anemia [Internet]. 2015 [citado marzo 2019]; 2015: 763576. Disponible en: https://www.hindawi. com/journals/anemia/2015/763576/. 


\section{Utilidad}

Diferencia a los pacientes con HIT (trombocitopenia inducida por heprina), de aquellos con otras causas de trombocitopenia.

\section{Descripción}

- Considere utilizar el sistema de puntuación 4Ts para estratificar el riesgo de HIT, en pacientes con trombocitopenia que se encuentran o se encontraban con agentes derivados de heparina.

\section{Parámetros}

\begin{tabular}{|c|c|c|}
\hline CRITERIO & CARACTERÍSTICAS & PUNTAJE \\
\hline Trombocitopenia & $\begin{array}{l}\text { Caída del recuento de plaquetas }>50 \% \\
\text { y nadir de plaquetas } \geq 20 \text {. } \\
\text { El recuento de plaquetas cae } 30-50 \% \text { o } \\
\text { nadir de plaquetas } 10-19 \text {. } \\
\text { Recuento de plaquetas caído }<30 \% \text { o } \\
\text { nadir plaquetario }<10 \text {. }\end{array}$ & 2 \\
\hline $\begin{array}{l}\text { Tiempo de caída } \\
\text { del recuento de } \\
\text { plaquetas }\end{array}$ & $\begin{array}{l}\text { Inicio claro entre los días } 5 \text { y } 10 \text { o caída } \\
\text { de plaquetas } \leq 1 \text { día (exposición previa } \\
\text { a la heparina dentro de los } 30 \text { días). } \\
\text { Consistente con la caída de los días } \\
5-10 \text {, pero no está claro; inicio después } \\
\text { del día } 10 \text { o caída } \leq 1 \text { día (exposición } \\
\text { previa a la heparina hace } 30 \text { a } 100 \\
\text { días). } \\
\text { El recuento de plaquetas cae }<4 \text { días } \\
\text { sin exposición reciente. }\end{array}$ & 0 \\
\hline
\end{tabular}




\begin{tabular}{|l|l|c|}
\hline & $\begin{array}{l}\text { Nueva trombosis o necrosis de la piel; } \\
\text { reacción sistémica aguda posterior a } \\
\text { IV bolo de heparina. }\end{array}$ & 2 \\
otras secuelas & $\begin{array}{l}\text { Trombosis recurrente o recurrente; } \\
\text { lesiones cutáneas no necrotizantes; } \\
\text { sospecha de trombosis (no probado). }\end{array}$ & 1 \\
\hline Ninguna. & 0 \\
\hline $\begin{array}{l}\text { Otras causas de } \\
\text { trombocitopenia }\end{array}$ & $\begin{array}{l}\text { Ninguno aparente } \\
\text { Posible }\end{array}$ & 2 \\
\hline
\end{tabular}

\section{Interpretación}

\section{ÍNDICE DE RETICULOCITOS INTERPRETACIÓN}

\begin{tabular}{|l|l|}
\hline$\leq 3$ & Baja probabilidad de HIT $\leq 5 \%$ \\
\hline $4-5$ & Probabilidad intermedia $14 \%$ \\
\hline 6-8 & Alta probabilidad $64 \%$ \\
\hline
\end{tabular}

\section{Bibliografía}

\section{Original}

1. Logk, juhld, warkentin te, sigouin cs, eichler p, greinacher a. Evaluación del puntaje clínico pretest (4 ts) para el diagnóstico de trombocitopenia inducida por heparina en dos contextos clínicos. J thromb haemost [Internet. 2006 [citado marzo 2019]; 4: 759-65. Disponible en: https:/ / www.ncbi.nlm.nih.gov/pubmed/16634744.

\section{Validación}

1. Vatanparast $\mathrm{r}$, lantz s, ward k, crilley pa, styler m. Evaluación de un sistema de puntuación pretest (4ts) para el diagnóstico de trombocitopenia inducida por heparina en un hospital universitario. Postgrado med [Internet]. 2012 nov [citado marzo 2019]; 124 (6): 36-42. Disponible en: https: / / www.ncbi.nlm.nih.gov/ pubmed/ 23322137. 
CÁCULOSY

PROHÓSTICAS

EN MEDICINA INTERNA 


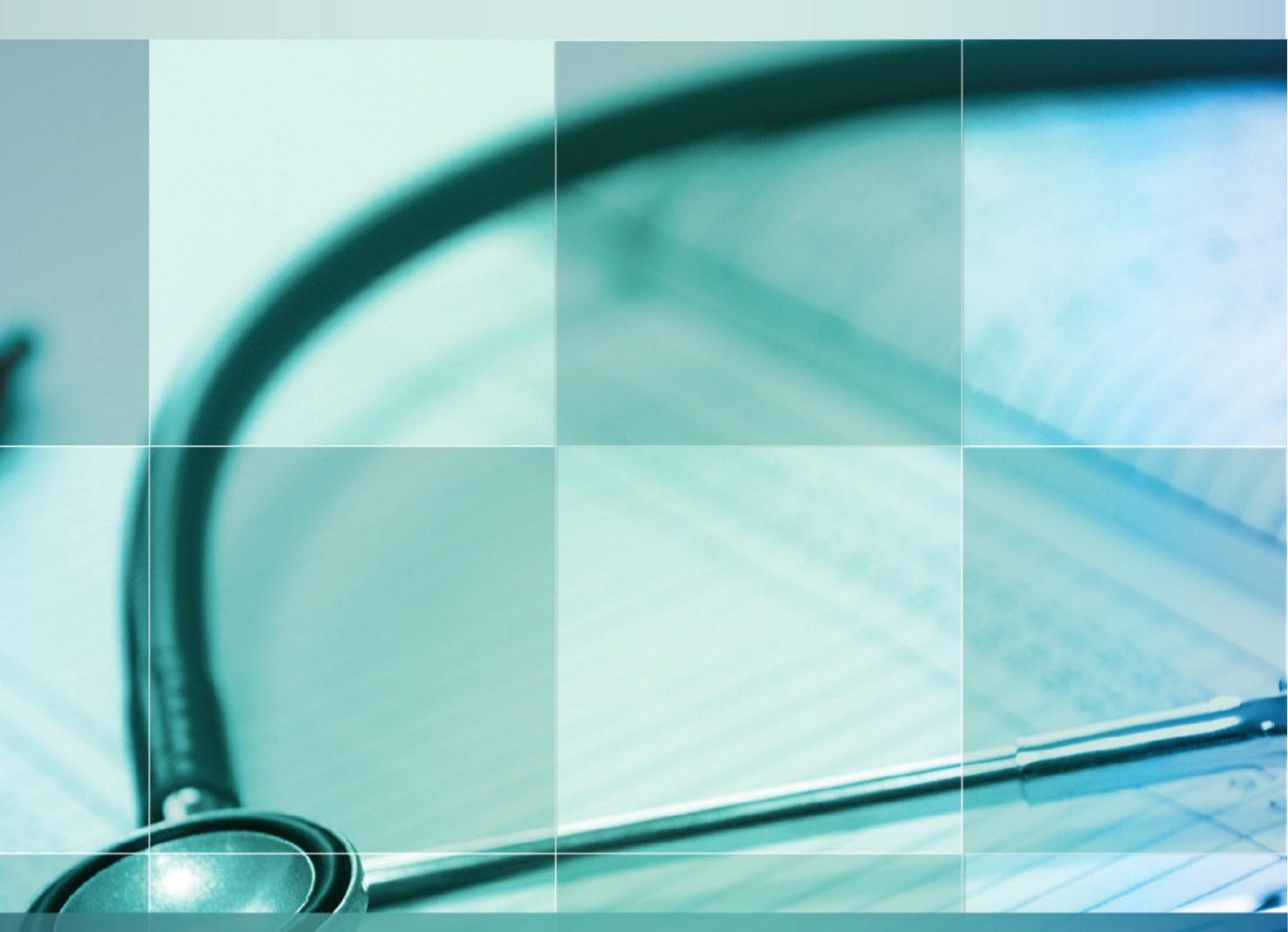

\section{OTRAS ESCALAS Y CÁLCULOS DE USO EN LA PRÁCTICA CLÍNICA}

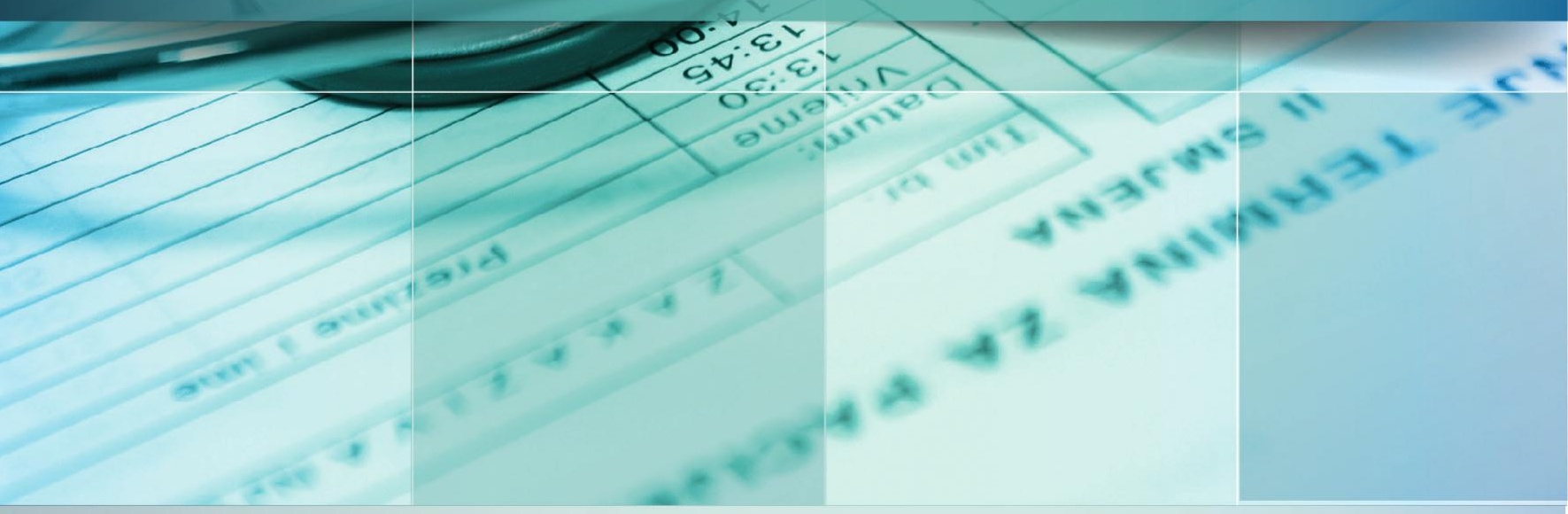




\section{Utilidad}

Identifica pacientes con riesgo de úlceras por presión.

\section{Parámetros}

\begin{tabular}{|c|c|c|c|c|}
\hline CRITERIO & 1 & 2 & 3 & 4 \\
\hline \multirow[b]{2}{*}{$\begin{array}{l}\text { Percepción } \\
\text { sensorial }\end{array}$} & $\begin{array}{l}\text { Completamente } \\
\text { limitada }\end{array}$ & Muy limitada & $\begin{array}{l}\text { Levemente } \\
\text { limitada }\end{array}$ & No alterada \\
\hline & $\begin{array}{l}\text { No responde ni } \\
\text { a estímulos do- } \\
\text { lorosos }\end{array}$ & $\begin{array}{l}\text { Responde } \\
\text { solamente a } \\
\text { estímulos do- } \\
\text { lorosos }\end{array}$ & $\begin{array}{l}\text { Responde a } \\
\text { órdenes ver- } \\
\text { bales }\end{array}$ & $\begin{array}{l}\text { Sin déficit } \\
\text { sensorial }\end{array}$ \\
\hline \multirow[b]{2}{*}{ Humedad } & $\begin{array}{l}\text { Completamente } \\
\text { húmeda }\end{array}$ & Muy húmeda & $\begin{array}{l}\text { Ocasional- } \\
\text { mente hú- } \\
\text { meda }\end{array}$ & $\begin{array}{l}\text { Raramente } \\
\text { húmeda }\end{array}$ \\
\hline & $\begin{array}{l}\text { Casi constante- } \\
\text { mente (sudor, } \\
\text { orina) }\end{array}$ & $\begin{array}{l}\text { Es necesario } \\
\text { el cambio de } \\
\text { sábanas por } \\
\text { turno }\end{array}$ & $\begin{array}{l}\text { Es necesario } \\
\text { el cambio } \\
\text { de sábanas } \\
\text { cada } 12 \text { ho- } \\
\text { ras }\end{array}$ & $\begin{array}{l}\text { Piel normal- } \\
\text { mente seca }\end{array}$ \\
\hline Actividad & En cama & En silla & $\begin{array}{l}\text { Camina } \\
\text { ocasional- } \\
\text { mente }\end{array}$ & $\begin{array}{l}\text { Camina con } \\
\text { frecuencia }\end{array}$ \\
\hline \multirow[b]{2}{*}{ Movilidad } & $\begin{array}{l}\text { Completamente } \\
\text { inmóvil }\end{array}$ & Muy limitada & $\begin{array}{l}\text { Ligeramen- } \\
\text { te limitada }\end{array}$ & $\begin{array}{l}\text { Sin limita- } \\
\text { ciones }\end{array}$ \\
\hline & $\begin{array}{l}\text { No realiza ni } \\
\text { ligeros cambios } \\
\text { de posición }\end{array}$ & $\begin{array}{l}\text { Realiza lige- } \\
\text { ros cambios } \\
\text { de forma oca- } \\
\text { sional }\end{array}$ & $\begin{array}{l}\text { Realiza lige- } \\
\text { ros cambios } \\
\text { de forma } \\
\text { frecuente }\end{array}$ & $\begin{array}{l}\text { Realiza } \\
\text { cambios de } \\
\text { forma autó- } \\
\text { noma }\end{array}$ \\
\hline \multirow[b]{2}{*}{ Nutrición } & Muy pobre & $\begin{array}{l}\text { Probable- } \\
\text { mente inade- } \\
\text { cuada }\end{array}$ & Adecuada & Excelente \\
\hline & $\begin{array}{l}\text { Rara vez come } \\
\text { más de un tercio } \\
\text { del plato }\end{array}$ & $\begin{array}{l}\text { Rara vez } \\
\text { come más de } \\
\text { la mitad del } \\
\text { plato }\end{array}$ & $\begin{array}{l}\text { Normal- } \\
\text { mente come } \\
\text { más de la } \\
\text { mitad del } \\
\text { plato }\end{array}$ & $\begin{array}{l}\text { Come la } \\
\text { mayoría de } \\
\text { los platos } \\
\text { enteros }\end{array}$ \\
\hline
\end{tabular}




\begin{tabular}{|l|l|l|l|l|}
\hline \multirow{2}{*}{$\begin{array}{l}\text { Fricción } \\
\text { y desliza- } \\
\text { miento }\end{array}$} & \begin{tabular}{l} 
Es un problema \\
\cline { 2 - 5 }
\end{tabular} & $\begin{array}{l}\text { Es un proble- } \\
\text { ma potencial }\end{array}$ & $\begin{array}{l}\text { Sin proble- } \\
\text { ma aparente }\end{array}$ & \\
& $\begin{array}{l}\text { lizarlo es impo- } \\
\text { sible }\end{array}$ & $\begin{array}{l}\text { Al movilizar- } \\
\text { lo, la piel se } \\
\text { deliza sobre } \\
\text { las sábanas } \\
\text { ligeramente }\end{array}$ & $\begin{array}{l}\text { Se mueve } \\
\text { autónoma- } \\
\text { mente }\end{array}$ & \\
\hline
\end{tabular}

Interpretación

\begin{tabular}{|c|c|}
\hline PUNTUACIÓN & NIVEL DE RIESGO \\
\hline$<12$ & Alto \\
\hline $\mathbf{1 2 - 1 4}$ & Moderado \\
\hline $\mathbf{1 5 - 1 6}$ & Bajo \\
\hline
\end{tabular}

\section{Bibliografía}

\section{Original/ Primaria}

1. Bergstrom N, Braden BJ, Laguzza A, Holman V. La escala de Braden para predecir el riesgo de dolor por presión. Nurs Res [Internet]. 1987 [citado marzo 2019]; 36 (4): 205-10. Disponible en: https: / / www.ncbi. nlm.nih.gov/pubmed/3299278.

\section{Validación}

1. Bergstrom N, Braden BJ. Validez predictiva de la escala de Braden entre sujetos blancos y negros. Nurs Res [Internet]. 2002 [citado marzo 2019]; 51 (6): 398-403. Disponible en: https: / / www.ncbi.nlm.nih.gov / pubmed / 12464760 .

2. Bergstrom N, Braden B, Kemp M, Champagne M, Ruby E. Predicción del riesgo de úlcera por presión: un estudio multisitio de la validez predictiva de la escala de Braden. Nurs Resp [Internet]. 1998 [citado marzo 2019] ; 47 (5): 261-9. Dsiponibl en: https: / / www.ncbi.nlm.nih. gov/pubmed/9766454.

3. Hyun S, Vermillion B, Newton C, et al. Validez predictiva de la escala de Braden para pacientes en unidades de cuidados intensivos. Am J Crit Care [Internet]. 2013 [citado marzo 2019]; 22 (6): 514-520. Disponible en: https: / / www.ncbi.nlm.nih.gov/pmc/articles / PMC4042540/. 


\section{ESCALA DE MORSE PARA RIESGO DE CAÍDA}

\section{Utilidad}

Es una herramienta que permite evaluar la probabilidad de que un paciente sufra una caída.

\section{Parámetros}

\begin{tabular}{|c|c|c|}
\hline CRITERIO & CARACTERÍSTICAS & PUNTAJE \\
\hline \multirow{2}{*}{$\begin{array}{l}\text { Antecedentes } \\
\text { de caída }\end{array}$} & No & 0 \\
\hline & Sí & 25 \\
\hline \multirow{2}{*}{$\begin{array}{l}\text { Diagnóstico } \\
\text { secundario }\end{array}$} & No & 0 \\
\hline & Sí & 15 \\
\hline \multirow{3}{*}{$\begin{array}{l}\text { Ayuda para } \\
\text { deambular }\end{array}$} & $\begin{array}{l}\text { Reposo en cama/asistencia de } \\
\text { enfermería }\end{array}$ & 0 \\
\hline & Bastón/muletas/andador & 15 \\
\hline & Se apoya en los muebles & 30 \\
\hline \multirow{2}{*}{ Vía venosa } & No & 0 \\
\hline & Sí & 20 \\
\hline \multirow{3}{*}{ Marcha } & Normal/inmovilizado/reposo en cama & 0 \\
\hline & Débil & 15 \\
\hline & Alterada requiere asistencia & 30 \\
\hline \multirow{2}{*}{ Estado mental } & Conciente de su capacidad y limitación & 0 \\
\hline & No conciente de sus limitaciones & 15 \\
\hline
\end{tabular}

\section{Interpretación}

\section{PUNTUACIÓN NIVEL DE RIESGO}

ACCIÓN

0-24

$$
\text { Sin riesgo }
$$

Cuidados enfermería 


\begin{tabular}{|l|l|l|}
\hline $\mathbf{2 5 - 5 0}$ & Riesgo moderado & $\begin{array}{l}\text { Implementar plan de } \\
\text { prevención de caídas } \\
\text { estándar }\end{array}$ \\
\hline $\mathbf{> 5 1}$ & Riesgo alto & $\begin{array}{l}\text { Implementar } \\
\text { especiales }\end{array}$ \\
\hline
\end{tabular}

\section{Bibliografía}

\section{Original/Primaria}

1. Morse, JM, Morse, RM, Tylko, SJ. Desarrollo de una escala para identificar al paciente propenso a las caídas. Canadian Journal on Aging [Internet]. 1989 [citado marzo 2019:] 8 (4), 366-377. Disponible en: https: / psycnet.apa.org/ record/1990-18744-001.

\section{Validacion}

1. O'Connell B, Myers H. La sensibilidad y especificidad de la escala de caída de Morse en un entorno de cuidados intensivos. J Clin Nurs [Internet]. 2002 [citado marzo 2019]. 11 (1): 134-6. Disponible en: https: / / www.ncbi.nlm.nih.gov/pubmed/11845750.

2. Aranda-Gallardo M, Morales-Asencio JM, Canca-Sanchez JC, et al. Instrumentos para evaluar el riesgo de caídas en pacientes hospitalizados agudos: una revisión sistemática y un metanálisis. BMC Health Service Res [Internet]. 2013 [citado marzo 2019]1; 13: 122. Disponible en: https: / / www.ncbi.nlm.nih.gov/ pubmed/23547708.

3. Modelo de Gestión de Información de los Formularios del Manual de Seguridad del Paciente para el Sistema Nacional de Salud. MSP 2016. Código: DNCSS-MSP-008, Versión: 1, Fecha: 01/08/2016. 


\section{ESCALA DE KARNOFSKY}

\section{Utilidad}

Herramienta utilizada para medir la capacidad de los pacientes con cáncer para realizar tareas rutinarias.

\section{Descripción}

- Cuantifica las capacidades funcionales de un paciente y el impacto de tratamientos como la quimioterapia, en sus capacidades funcionales básicas.

- El estado de desempeño está altamente correlacionado con el tiempo de supervivencia y la necesidad de servicios en el hogar.

- También puede ayudar a predecir la capacidad de prosperar con un tratamiento invasivo e intensivo.

\section{Parámetros}

\begin{tabular}{|l|c|}
\hline \multicolumn{1}{|c|}{ CARACTERÍSTICAS } & PUNTAJE \\
\hline Normal no hay quejas; no hay evidencia de enfermedad & 100 \\
\hline $\begin{array}{l}\text { Capaz dellevar a cabo la actividad normal; signos menores } \\
\text { o síntomas de enfermedad. }\end{array}$ & 90 \\
\hline $\begin{array}{l}\text { Actividad normal con esfuerzo; algunos signos o síntomas } \\
\text { de enfermedad. }\end{array}$ & 80 \\
\hline $\begin{array}{l}\text { Se preocupa por sí mismo; incapaz de realizar una } \\
\text { actividad normal o hacer un trabajo activo. }\end{array}$ & 70 \\
\hline $\begin{array}{l}\text { Requiere asistencia ocasional, pero puede atender la } \\
\text { mayoría de sus necesidades personales. }\end{array}$ & 60 \\
\hline $\begin{array}{l}\text { Requiere asistencia considerable y atención médica } \\
\text { frecuente. }\end{array}$ & 50 \\
\hline Discapacitado; requiere atención y asistencia especial. & 30 \\
\hline $\begin{array}{l}\text { Gravemente discapacitado; se indica ingreso hospitalario } \\
\text { aunque la muerte no es inminente. }\end{array}$ & 20 \\
\hline $\begin{array}{l}\text { Muy enfermo; ingreso hospitalario necesario; tratamiento } \\
\text { de apoyo activo necesario. }\end{array}$ & 0 \\
\hline Moribundo; irreversible & \\
\hline Muerto & \\
\hline
\end{tabular}




\section{Interpretación}

\begin{tabular}{|c|c|c|c|}
\hline PUNTUACIÓN & NIVEL DE RIESGO & $\begin{array}{c}\text { ESTADO DE RENDIMIENTO } \\
\text { ECOG }\end{array}$ & PTS. \\
\hline 100 & \multirow{3}{*}{$\begin{array}{ll}\text { Capaz de realizar } & \\
\text { una } & \text { actividad } \\
\text { normal y de } \\
\text { trabajar; no se } \\
\text { necesita } & \text { atención } \\
\text { especial. } & \end{array}$} & \multirow{2}{*}{$\begin{array}{l}\text { 0: completamente activo, } \\
\text { capaz de llevar a cabo } \\
\text { todo el rendimiento } \\
\text { previo a la enfermedad } \\
\text { sin restricciones. }\end{array}$} & 100 \\
\hline 90 & & & 90 \\
\hline 80 & & \multirow{2}{*}{$\begin{array}{l}\text { 1: Restringido en } \\
\text { actividades físicamente } \\
\text { extenuantes, pero } \\
\text { ambulatorio y capaz } \\
\text { de realizar trabajos } \\
\text { de naturaleza ligera o } \\
\text { sedentaria; por ejemplo, } \\
\text { trabajo de casa ligero, } \\
\text { trabajo de oficina. }\end{array}$} & 80 \\
\hline 70 & \multirow{3}{*}{$\begin{array}{l}\text { Incapaz de } \\
\text { trabajar; capaz } \\
\text { de vivir en casa y } \\
\text { cuidar la mayoría } \\
\text { de sus necesidades } \\
\text { p e r o n a les ; } \\
\text { cantidad variable } \\
\text { de asistencia } \\
\text { necesaria. }\end{array}$} & & 70 \\
\hline 60 & & \multirow{2}{*}{$\begin{array}{l}\text { 2: Ambulatorio y capaz } \\
\text { de cuidarse solo, pero sin } \\
\text { poder realizar ninguna } \\
\text { actividad laboral; más del } \\
50 \% \text { de las horas de vigilia. }\end{array}$} & 60 \\
\hline 50 & & & 50 \\
\hline 40 & \multirow{5}{*}{$\begin{array}{l}\text { Incapaz de } \\
\text { cuidarse a sí } \\
\text { mismo; requiere } \\
\text { un equivalente } \\
\text { de atención } \\
\text { institucional u } \\
\text { hospital a ri a ; } \\
\text { la enfermedad } \\
\text { puede estar } \\
\text { prog r e s a n d o } \\
\text { rápidamente. }\end{array}$} & \multirow{2}{*}{$\begin{array}{l}\text { 3: Capaz de autocuidado } \\
\text { limitado; confinado a la } \\
\text { cama o silla, más del } 50 \% \\
\text { de las horas de vigilia. }\end{array}$} & 40 \\
\hline 30 & & & 30 \\
\hline 20 & & \multirow{2}{*}{$\begin{array}{l}\text { 4:C o m p l e t a m e n t e } \\
\text { deshabilitado; r no } \\
\text { puede llevar ningún } \\
\text { autocuidado; totalmente } \\
\text { confinado a la cama o silla. }\end{array}$} & 20 \\
\hline 10 & & & 10 \\
\hline 0 & & 5: Muerto & 0 \\
\hline
\end{tabular}

ECOG: escala de estado funcional en pacientes con cáncer. 


\section{Bibliografía}

\section{Original/Primaria}

1. Karnofsky DA Burchenal JH. (1949). ‘La evaluación clínica de los agentes quimioterapéuticos en el cáncer'. MacLeod CM (Ed), Evaluación de agentes quimioterapéuticos. Columbia Univ Press. Página 196.

\section{Validación}

1. Crooks, V, Waller S, et al. El uso de la Escala de rendimiento de Karnofsky para determinar los resultados y el riesgo en pacientes ambulatorios geriátricos. J Gerontol [Internet]. 1991 [citado marzo 2019]; 46: M139-M144. Dsiponible en https://www.ncbi.nlm.nih. gov/pubmed/2071835.

2. Hollen PJ , Gralla RJ, Kris MG, Cox C. Medición de la calidad de vida en pacientes con cáncer de pulmón en ensayos multicéntricos de nuevas terapias. Evaluación psicométrica de la Escala de Síntomas de Cáncer de Pulmón. Cáncer [Internet]. 1994 \{citado marzo 2019] ; 73 (8): 208798. Disponible en: https: / / www.ncbi.nlm.nih.gov/ pubmed/8156514.

3. O'Toole DM, Golden AM. Evaluación de pacientes con cáncer para el potencial de rehabilitación. West J Med [Internet]. 1991 [citado marzo 2019]; 155 (4): 384-387. Disponible en https://www.ncbi.nlm.nih. gov/pmc/articles/PMC1003019/. 


\section{CÁLCULOS HEMODINÁMiCOS}

Los parámetros hemodinámicos son fundamentales para el diagnóstico, tratamiento, seguimiento y pronóstico de las enfermedades cardiovasculares, sobre todo para las cardiopatías congénitas y la hipertensión pulmonar. La medición de los parámetros hemodinámicos requiere cateterismo cardíaco; sin embargo, con el desarrollo de la ecocardiografía la mayoría de esos parámetros hemodinámicos se pueden estimar por ecocardiografía, siendo el uso del cateterismo cardíaco en casos específicos y para intervencionismo. En ese sentido se expone los parámetros más utilizados en la práctica clínica.

\section{Gasto Cardíaco:}

Es la cantidad de flujo sanguíneo expulsado por el corazón, hacia la circulación sanguínea por unidad de tiempo, es expresado por litros/ minuto. Cuando el gasto cardíaco se indexa a la superficie corporal (sc) se conoce como índice cardíaco $(1 / \mathrm{min} / \mathrm{m} 2 \mathrm{sc})$.

$$
\text { GC }(1 / \mathrm{min}): \frac{\text { Consumo de oxígeno }(\mathrm{ml} / \mathrm{min})}{\text { Concentración oxígeno aórtico }- \text { pulmonar }(\mathrm{ml} / 100 \mathrm{ml}) \times 10}
$$

1. El gasto cardíaco se determina por cateterismo cardíaco por el método de Fick, que se basa en determinación del consumo de oxígeno y la

$$
\text { IC: } \frac{\mathrm{GC}(1 / \mathrm{min})}{\text { superficie corporal }(\mathrm{m} 2)}
$$

diferencia arterio-venosa de la concentración de oxígeno.

También se determina a partir del método de termodilución, a través del cateterismo derecho (Swan-Ganz), acoplado a un sistema computarizado.

IC: índice cardíaco

GC: gasto cardíaco.

Valor de referencia del índice cardíaco por cateterismo: 2.5-3.5 1/min/ $\mathrm{m} 2$.

2. Por ecocardiografía se determina de la siguiente manera. 
GC: volumen sistólico $x$ frecuencia cardíaca

Volumen sistólico (VS): Área tsvi $\left(\mathrm{cm}^{2}\right)$ x IVT tsvi

$\prod \mathrm{r}^{2} \mathrm{x}$ IVTtsvi. $\quad \mathrm{r}: \frac{1}{2}$ Dtsvi

\subsection{4/ 4 x Dtsvi ${ }^{2} \times$ IVTtsvi \\ $0.785 \times$ Dtsvi $^{2} \times$ IVT tsvi}

Dtsvi: diámetro del tracto de salida del ventrículo izquierdo.

IVT: integral velocidad tiempo: es la distancia recorrida por la sangre en cada latido.

Valores de referencia: Volumen sistólico: 50-90 ml

Gasto cardíaco: 4-7 $\mathrm{ml} / \mathrm{min}$

Índice cardíaco: $2.5-4.51 / \mathrm{min} / \mathrm{m}^{2}$

Una de las aplicaciones clínicas fundamentales de la medida de flujo sistólico, es la relación flujo pulmonar sobre el flujo aórtico (sistémico), conocido como Qp/Qs, que se usa para determinar la repercusión hemodinámica de un cortocircuito cardíaco (cardiopatías congénitas: CIA, CIV, PCA) y ayuda a la toma de decisión para la corrección del cortocircuito.

\section{Resistencias vasculares:}

Se mide por cateterismo cardíaco, la unidad de medida es: unidades wood ( $\mathrm{mmhg} / 1 / \mathrm{min})$, también se expresa en dynes $/ \mathrm{s} / \mathrm{cm}^{-5}$ donde 1 unidad Wood es igual a 80 dynes $/ \mathrm{s} / \mathrm{cm}^{-5}$

RVS: $\frac{80 \text { (Presión media aórtica - Presión media AD (mmhg)) }}{\text { Gasto cardíaco }(1 / \mathrm{min})}$

\section{Resistencia vascular sistémica.}

Es útil en la determinación de la causa de hipotensión arterial, típicamente 
se encuentra disminuida en la sepsis y aumentada en el shock cardiogénico.

Valor de referencia: $800-1400$ dynes $/ \mathrm{s} / \mathrm{cm}^{-5}$

RVP: $\frac{80 \text { (Presión media arteria pulmonar - Presión media AI o PCP }(\mathrm{mmhg}))}{\text { Gasto cardíaco }(1 / \mathrm{min})}$

\section{Resistencia vascular pulmonar.}

Se mide en pacientes con hipertensión pulmonar y en caso de transplante cardíaco.

Valor de referencia: 25-125 dynes $/ \mathrm{s} / \mathrm{cm}^{-5}$

AD: aurícula derecha.

AI: aurícula izquierda.

PCP: presión de enclavamiento capilar pulmonar.

RVP: $\frac{\text { Velocidad máxima de la regurgitación tricúspide }}{\text { IVT tsvd }} \times 10+0.16$

En la práctica clínica se puede estimar la resistencia vascular pulmonar por ecocardiografía. Se basa en el conocimiento que la resistencia vascular pulmonar se relaciona en forma directa con la variación de la presión y en forma inversa con el flujo pulmonar, así lo expresa la ecuación de regresión de Abbas la que genera la mayor concordancia con la resistencia vascular pulmonar determinada en forma invasiva.

IVT: integral velocidad tiempo. Tsvd: tracto de salida del ventrículo derecho.

Valor normal: es menor de 2 unidades Wood.

\section{Presiones pulmonares:}

Las presiones sistólicas de la arteria pulmonar, presión media de la arteria pulmonar, presión diastólica de la arteria pulmonar y los gradientes deben medirse por cateterismo cardíaco para el diagnóstico, tratamiento 
y pronóstico de la hipertensión pulmonar. Sin embargo, los mismos parámetros se pueden estimar por ecocardiografía, siendo el uso del cateterismo cardíaco en casos específicos.

Con la aplicación clínica de la ecocardiografía se estima las presiones pulmonares de la siguiente forma.

\section{Presión sistólica de la arteria pulmonar (PSAP)}

Se determina a partir de la ecuación de Bernoulli.

Gradiente de Presión: 4 x Vmáx ${ }^{2}$

PSAP: 4 x VIT ${ }^{2}+$ Presión AD (En ausencia de estenosis pulmonar).

PSAP: presión sistólica de la arteria pulmonar.

VT: velocidad de insuficiencia tricuspídea.

AD: aurícula derecha.

La presión de la aurícula derecha se determina a través del colapso inspiratorio de la vena cava inferior.

2. Presión media de la arteria pulmonar (PMAP).

Método de Mahan:

PMAP: 79 - (TAC x 0.45)

TAC: Tiempo de Aceleración.

Método de Masayuma:

PMAP: 4 x Vip² + Presión de AD (Vp: Velocidad máxima insuficiencia pulmonar).

Método Chemla: PMAP: PSAP x $0.61+2$.

3. Presión diastólica de la arteria pulmonar (PDAP) .

$$
\text { PDAP: } 4 \times \mathrm{Vpt}^{2}+\mathrm{PAD}
$$

Vpt: velocidad de regurgitación pulmonar tardía.

4. Gradiente de presión diastólico (GPD). 
GPD: PDAP - PCP (presión de enclavamiento capilar pulmonar).

La medición de esas presiones asociado a la presión de enclavamiento capilar pulmonar y la resistencia vascular pulmonar por cateterismo cardíaco, permite realizar la clasificación hemodinámica de la hipertensión pulmonar.

\section{Clasificación hemodinámica de la hipertensión pulmonar}

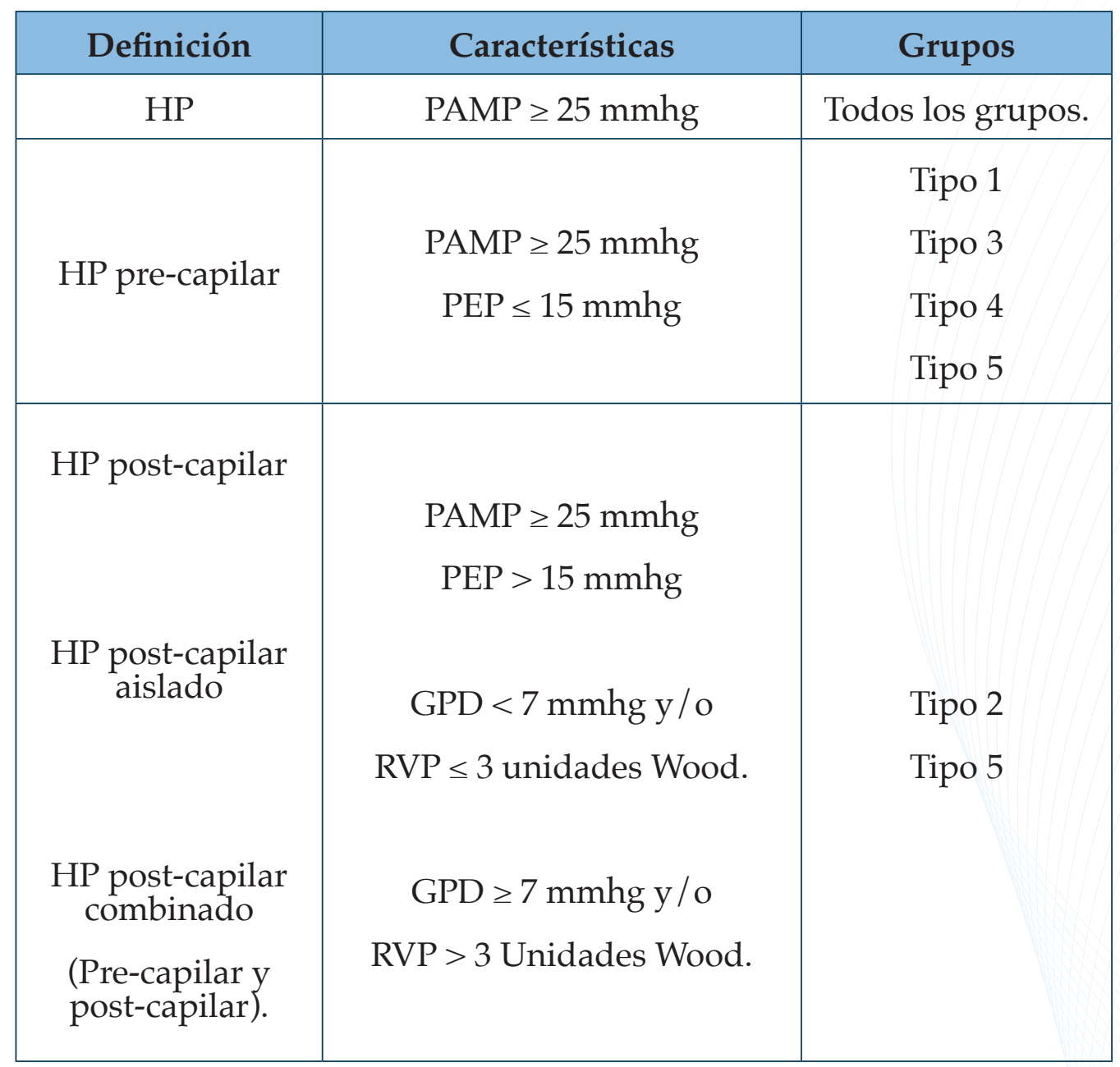

Clasificación de la HP según valores de la presión pulmonar.

1. Leve: PAMP: 25-35 mmhg

PSVD: $35-49$ mmhg

2. Moderado: PAMP: $36-45 \mathrm{mmhg}$ 


$$
\text { PSVD: 50-69 mmhg }
$$

3. Severa: PAMP: $>45 \mathrm{mmhg}$

FE: $\frac{\text { Volumen telediastólico }- \text { Volumen telesistólico }}{\text { Volumen telediastólico }} \times 100$

PSVD: $\geq 70$ mmhg.

\section{Fracción de eyección del ventrículo izquierdo.}

Es uno de los parámetros más utilizado para evaluar la función sistólica del ventrículo izquierdo.

Se determina tanto por cateterismo cardíaco como por ecocardiografía.

Valores de referencia.

\begin{tabular}{|l|l|l|l|l|}
\hline & Normal & $\begin{array}{l}\text { Disfunción } \\
\text { leve }\end{array}$ & $\begin{array}{l}\text { Disfunción } \\
\text { moderada }\end{array}$ & $\begin{array}{l}\text { Disfunción } \\
\text { severa }\end{array}$ \\
\hline FE Hombres (\%) & $52-72$ & $41-51$ & $30-40$ & $<30$ \\
\hline FE Mujeres (\%) & $54-74$ & $41-53$ & $30-40$ & $<30$ \\
\hline
\end{tabular}

\section{Bibliografía}

1. Mitchell, Andrew R.J.; West, Nick E.J.; Leeson, Paul; Banning, Adrian P. Cardiac Catheterization and Coronary Intervention, 1st Edition, 2008.

2. Baim, Donald S. Grossman's Cardiac Catheterization, Angiography, \& Intervention, 7th Edition, 2006.

3. Aviles, Ronnier J.; Askari, Arman T.; Messerli, Adrian W.; Penn, Marc S.; Topol, Eric J: Introductory Guide to Cardiac Catheterization, 1st Edition, 2004.

4. Catherine Otto, Pulmonary Hypertension En: The practice of clinical echocardiography, Fifth editión 2014.

5. L. Alvarez, M.A. Garcia-Fermandez, J.L. Zamorano. Hemodinamica, 
En: Procedimiento en ecocardiografía, Mcgram-Hill, Interamericana España, 2006.

6. ESC/ERS Guidelines for the diagnosis and treatment of pulmonary hypertension, 2015

7. Stuart Rich. Pulmonary hypertension. En: Braunwald heart diseases, 10 edition, 2015.

8. Feigenbaum. H. Hemodinamica, En: Feigenbaum Ecocardiografia, Editorial médica panamerica, 2006.

9. Recommendations for Cardiac Chamber Quantification by Echocardiography in Adults: An Update from the American Society of Echocardiography and the European Association of Cardiovascular Imaging, 2015. 
CÁCULOSY

PROHÓSTICAS

EN MEDICINA INTERNA 


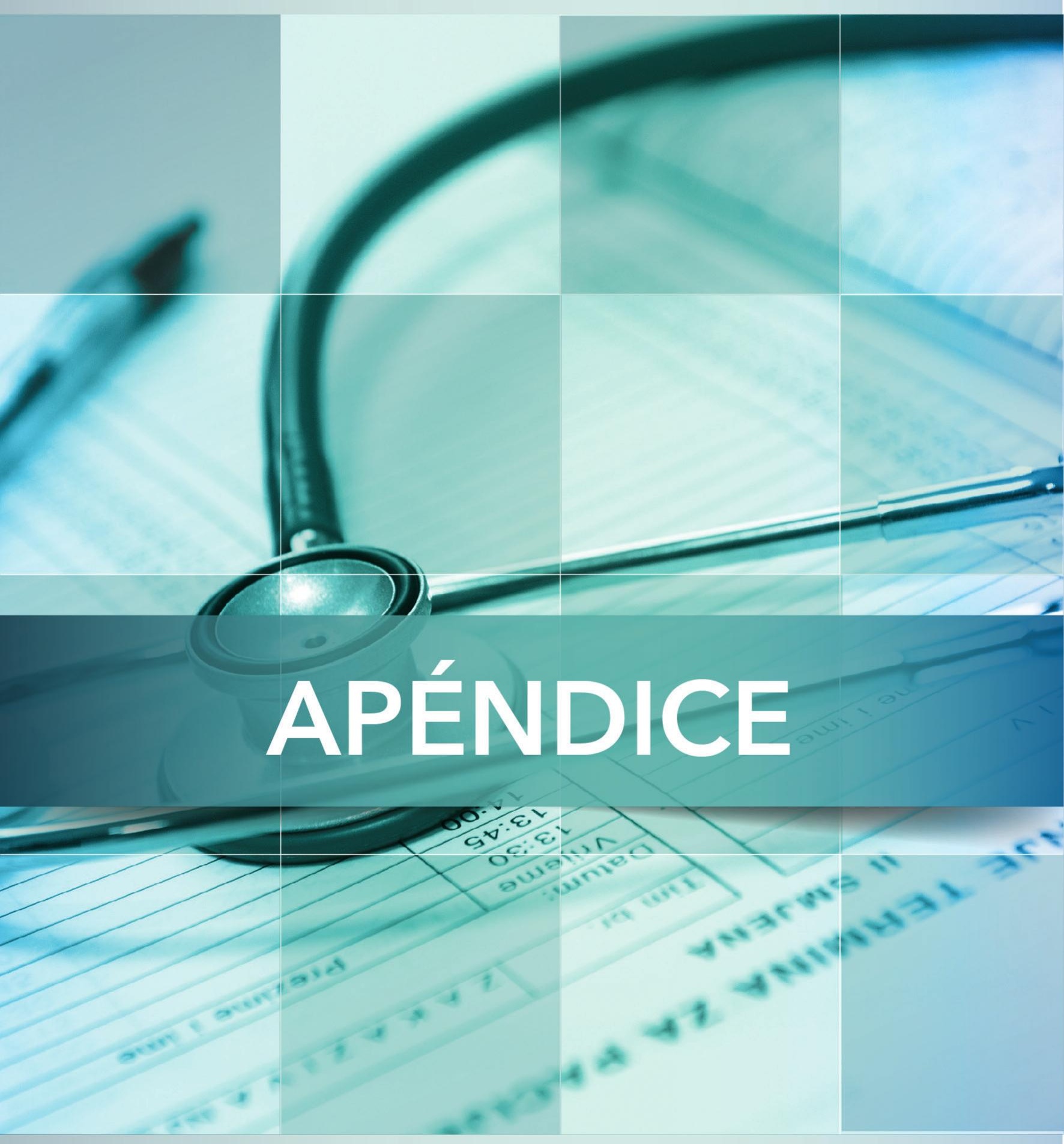




\section{APÉNDICE I.}

CÁLCULOS Y ESCALAS EN LA VALORACIÓN NUTRICIONAL DEL ADULTO Y ADULTO MAYOR

MsC. Johanna Villacrés C.

Nutricionista-Dietista. Espoch

\section{Valoración nutricional del adulto}

La valoración nutricional debería formar parte de la evaluación clínica de los individuos, que permite no solo determinar su estado de nutrición, sino también valorar los requerimientos nutricionales, predecir la posibilidad de presentar riesgos y evaluar la eficacia de una determinada terapia nutricional.

\section{PARÁMETROS ANTROPOMÉTRICOS Y COMPOSICIÓN CORPORAL}

Peso

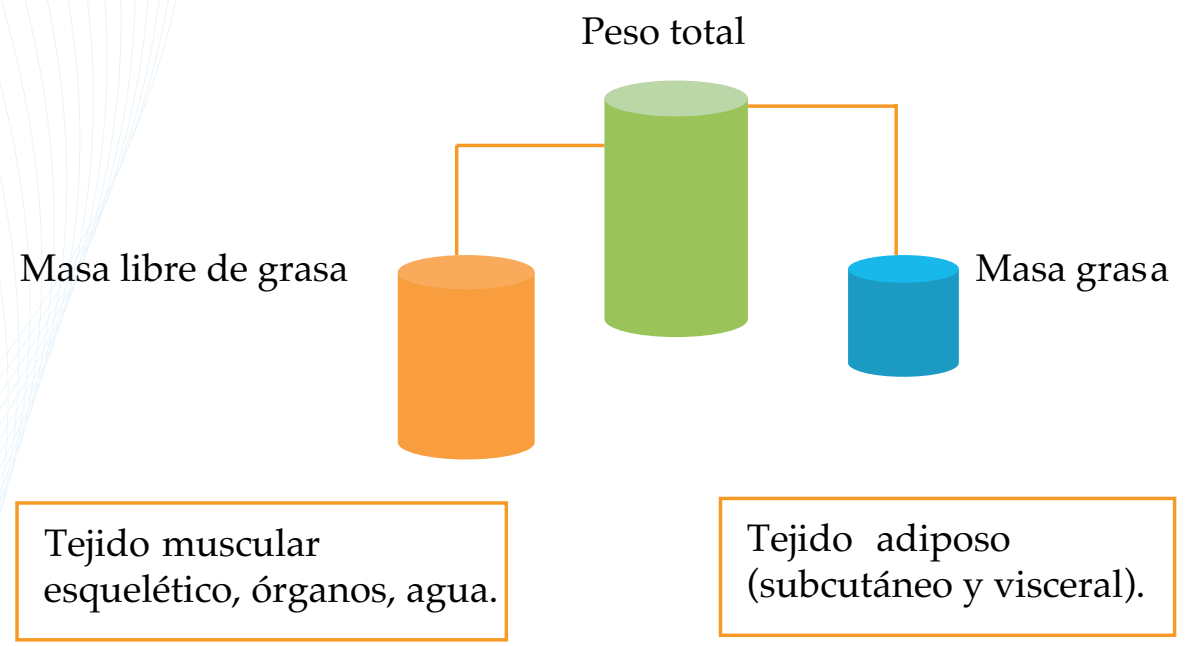

\section{Peso ideal:}

\section{Estimar:}

- Tabla metropolitan life insurance (1983).

- Tabla sociedad española de diabetes (1993). 


\begin{tabular}{|c|c|c|c|}
\hline \multicolumn{4}{|c|}{ CÁLCULO DEL PESO IDEAL } \\
\hline F. Lorentz & $\mathrm{P}=[\mathrm{T}-100]-$ & $\begin{array}{r}\frac{(T-15}{4} \\
]-0,25(T\end{array}$ & $-150)$ \\
\hline $\begin{array}{l}\text { Fórmula metropolitan life } \\
\text { insucarence company }\end{array}$ & \multicolumn{3}{|c|}{$\mathrm{PI}=50+[0.75 x($ talla -150$)]$} \\
\hline Fórmula de Moreiro & \multicolumn{3}{|c|}{$\begin{array}{l}\text { Mujer: } 54+[1,3 \times(\mathrm{T}-150)] / 2,5 \\
\text { Varón:59+ [1,3 x (T-150)] / 2,5 }\end{array}$} \\
\hline $\begin{array}{l}\text { Peso ideal basado en IMC } \\
\text { promedio e ideal }\end{array}$ & \multicolumn{3}{|c|}{$\begin{array}{l}\text { PI: } 21.7 \text { X TALLA }(\mathrm{m})^{2} \\
\text { Determinación de la complexión. }\end{array}$} \\
\hline \multirow{7}{*}{$\begin{array}{l}\text { Determinación de la } \\
\text { complexión. }\end{array}$} & \multicolumn{3}{|c|}{ Contextura $=\frac{\text { Altura }(\mathrm{cm})}{\text { C.de la muñeca }}$} \\
\hline & \multicolumn{3}{|c|}{ CONTEXTURA FÍSICA } \\
\hline & Contextura & Varones & Mujeres \\
\hline & Pequeña & $>10.4$ & $>11.0$ \\
\hline & Mediana & $9,6-10,4$ & 10.1-11.0 \\
\hline & Grande & $<9.6$ & $<10.1$ \\
\hline & \multicolumn{3}{|c|}{$\begin{array}{l}\text { Contextura corporal media: peso ideal } \\
\text { queda igual. } \\
\text { Contextura grande: al peso ideal se } \\
\text { aumenta } 5 \% \text {. } \\
\text { Contextura pequeña: peso ideal se quita } \\
5 \% \text {. }\end{array}$} \\
\hline
\end{tabular}




\section{Pérdida de peso}

Valoración del estado nutricional por la pérdida de peso en un período de tiempo

$\%$ pp=[peso habitual(kg)-peso actual(kg)/peso habitual] $\times 100$

\begin{tabular}{|l|c|c|c|}
\hline Tiempo & 1 mes & 3 meses & 6 meses \\
\hline Pérdida significativa & $5 \%$ & $7,5 \%$ & $10 \%$ \\
\hline Pérdida severa & $\mathbf{7 5 \%}$ & $>\mathbf{7 , 5}$ & $>\mathbf{1 0} \%$ \\
\hline
\end{tabular}

\section{Talla}

La talla de un adulto joven es estable, por tanto la evaluación debe estar encaminada a establecer la relación existente entre la talla que alcanzó el individuo y el peso.

\section{Determinación del índice de masa corporal (IMC)}

Representa en la actualidad uno de los índices más utilizados para el caso de los adultos, ya que describe el peso relativo para la estatura y está correlacionado de modo significativo con el contenido total de grasa del individuo.

$$
\mathrm{IMC}=\frac{\mathrm{IMC}=}{\text { Talla }\left(\mathrm{m}^{2}\right)}
$$

\section{Puntos de corte}

\begin{tabular}{|l|l|}
\hline IMC $\left(\mathbf{k g} / \mathbf{~}^{\mathbf{2}}\right)$ & Interpretación \\
\hline$<18.50$ & Bajo peso \\
\hline $18.5-24.99$ & Normal \\
\hline $25.0-29.9$ & Sobrepeso \\
\hline $30.0-34.9$ & Obesidad grado 1 \\
\hline $35.0-39.9$ & Obesidad grado 2 \\
\hline$>40.0$ & Obesidad grado 3 \\
\hline NOM manejo de la obesidad & \\
\hline$>27.0$ & Obesidad \\
\hline$>25.0$ & $\begin{array}{l}\text { Obesidad adultos de talla baja (mujer }< \\
1.5 \mathrm{~m}, \text { varón }<1.6 \mathrm{~m}) .\end{array}$ \\
\hline
\end{tabular}


Tomado de: NIH. Clinical Guidelines Identification, Evaluation, and Treatment of Overweight and Obesity in Adults. WHO. Obesity: Preventing and managing the global epidemic. 98. NOM-174-SSA1-98.

\section{Obesidad}

\section{Diagnóstico:}

- IMC

- \% Masa grasa

- Circunferencia abdominal.

\section{Determinación del porcentaje de Masa grasa (MG)}

La masa grasa representa la reserva energética del organismo; su evaluación a partir de pliegues cutáneos representa la cantidad de grasa localizada en el tejido adiposo subcutáneo, considerando que es representativo de la grasa corporal total.

Cálculo del \% MG a partir de la densidad corporal.

Ecuaciones para determinar la densidad corporal.

\begin{tabular}{|c|c|}
\hline $\begin{array}{l}\text { Rango de edad } \\
\text { (años) }\end{array}$ & Ecuación \\
\hline \multicolumn{2}{|l|}{ VARONES: } \\
\hline 17-19 & Densidad corporal $=1.1620-\left[0.0630 \times(\log \Sigma)^{\star}\right]$ \\
\hline $20-29$ & Densidad corporal $=1.1631-[0.0632 \times(\log \Sigma)]$ \\
\hline 30-39 & Densidad corporal $=1.1422-[0.0544 \times(\log \Sigma)]$ \\
\hline $40-49$ & Densidad corporal $=1.1620-[0.0700 \times(\log \Sigma)]$ \\
\hline $50+$ & Densidad corporal $=1.1715-[0.0779 \times(\log \Sigma)]$ \\
\hline \multicolumn{2}{|l|}{ MUJERES: } \\
\hline $17-19$ & Densidad corporal $=1.1549-[0.0678 \times(\log \Sigma)]$ \\
\hline $20-29$ & Densidad corporal $=1.1599-[0.0717 \times(\log \Sigma)]$ \\
\hline $30-39$ & Densidad corporal $=1.1423-[0.0632 \times(\log \Sigma)]$ \\
\hline $40-49$ & Densidad corporal $=1.1333-[0.0612 \times(\log \Sigma)]$ \\
\hline $50+$ & Densidad corporal $=1.1339-[0.0645 \times(\log \Sigma)]$ \\
\hline
\end{tabular}


Tomado de: Body fat assessment from total body density and its estimation from skinfold thickness: Measurements on 481 men and women aged 1672 years. British Journal of Nutrition 1974; 32:77-97.

${ }^{*} \Sigma=$ Suma de los pliegues tricipital, subescapular, suprailiaco y bicipital. A través del cálculo de la densidad corporal, se obtiene el porcentaje de grasa corporal aplicando la siguiente ecuación.

SIRI: $\%$ grasa $=\frac{4,95-4,50 \times 100}{\mathrm{D}}$ SIRI: $\%$ grasa $=(4.95 / \mathrm{D})-450$

BROZEK: $\%$ grasa $=\frac{4,570-4,142 \times 100}{\mathrm{D}} \quad$ BROZEK: $\%$ grasa $=(457 / \mathrm{D})-414$

Fórmula de Deurenberg y col.

$\%$ de Masa grasa $=1,2(\mathrm{IMC})+0,23($ edad $)-10,8($ sexo $)-5,4$

Sexo femenino $=0 /$ Sexo masculino $=1$

Interpretación del porcentaje de grasa corporal total:

\begin{tabular}{|l|l|l|}
\hline$\%$ grasa varones & $\%$ grasa Mujeres & \multicolumn{1}{c|}{ Interpretación } \\
\hline$\leq 5$ & $\leq 8$ & No saludable (muy bajo) \\
\hline $6-15$ & $9-23$ & Aceptable (bajo) \\
\hline $16-24$ & $24-31$ & Aceptable (alto) \\
\hline$\geq 25$ & $\geq 32$ & $\begin{array}{l}\text { No saludable - obesidad (muy } \\
\text { alto) }\end{array}$ \\
\hline
\end{tabular}

Para obtener el peso de la grasa corporal total (en kilogramos), se utiliza la siguiente formula:

Grasa corporal total $=\frac{\text { Peso actual } \times \% \text { de grasa corporal }}{100}$ 


\section{Determinación de la masa grasa:}

\begin{tabular}{|c|l|}
\hline $\begin{array}{c}\text { Medición del pliegue } \\
\text { cutáneo sub escapular } \\
\text { (PCSe). }\end{array}$ & $\begin{array}{l}\text { El pliegue subescapular se utiliza para } \\
\text { estimar las reservas energéticas y por } \\
\text { lo tanto la masa grasa corporal de un } \\
\text { individuo. } \\
\text { Pierde validez en sujetos con IMC >=35 } \\
\text { por la dificultad en la medición. }\end{array}$ \\
\hline $\begin{array}{c}\text { Categorías según MG } \\
\text { (puntos de corte). }\end{array}$ & $\begin{array}{l}\text { p25-p75 MG normal } \\
>\text { p7590 Exceso leve de MG }\end{array}$ \\
\hline p90-p95 Exceso moderado de MG \\
\hline
\end{tabular}

\section{Varones}

\begin{tabular}{|c|c|c|c|c|c|c|c|c|c|}
\hline \multirow{2}{*}{$\begin{array}{c}\text { Edad } \\
\text { (años) }\end{array}$} & \multicolumn{8}{|c|}{ Percentil ( pliegue subescapular en mm) } \\
\cline { 2 - 10 } & $\mathbf{5}$ & $\mathbf{1 0}$ & $\mathbf{1 5}$ & $\mathbf{2 5}$ & $\mathbf{5 0}$ & $\mathbf{7 5}$ & $\mathbf{8 5}$ & $\mathbf{9 0}$ & $\mathbf{9 5}$ \\
\hline 18.0 a 24.9 & 6.0 & 7.0 & 7.0 & 8.0 & 11.0 & 16.0 & 20.0 & 24.0 & 30.0 \\
\hline 25.0 a 29.9 & 7.0 & 7.0 & 8.0 & 9.0 & 13.0 & 20.0 & 24.5 & 26.5 & 31.0 \\
\hline 30.0 a 34.9 & 7.0 & 8.0 & 9.0 & 11.0 & 15.5 & 22.0 & 25.5 & 29.0 & 33.0 \\
\hline 35.0 a 39.9 & 7.0 & 8.0 & 9.5 & 11.0 & 16.0 & 22.5 & 25.5 & 28.0 & 33.0 \\
\hline 40.0 a 44.9 & 7.0 & 8.0 & 9.0 & 11.5 & 16.0 & 22.0 & 25.5 & 29.5 & 33.0 \\
\hline 45.0 a 49.9 & 7.0 & 8.0 & 9.5 & 11.5 & 17.0 & 23.5 & 27.0 & 30.0 & 34.5 \\
\hline 50.0 a 54.9 & 7.0 & 8.0 & 9.0 & 11.5 & 16.0 & 22.5 & 26.5 & 29.5 & 34.0 \\
\hline 55.0 a 59.9 & 6.5 & 8.0 & 9.5 & 11.5 & 16.5 & 23.0 & 26.0 & 28.5 & 32.0 \\
\hline
\end{tabular}

\section{Mujeres}

\begin{tabular}{|c|c|c|c|c|c|c|c|c|c|}
\hline \multirow{2}{*}{$\begin{array}{c}\text { Edad } \\
\text { (años) }\end{array}$} & \multicolumn{8}{|c|}{ Percentil ( pliegue subescapular en mm) } \\
\cline { 2 - 10 } & $\mathbf{5}$ & $\mathbf{1 0}$ & $\mathbf{1 5}$ & $\mathbf{2 5}$ & $\mathbf{5 0}$ & $\mathbf{7 5}$ & $\mathbf{8 5}$ & $\mathbf{9 0}$ & $\mathbf{9 5}$ \\
\hline 18.0 a 24.9 & 6.5 & 7.0 & 8.0 & 9.5 & 13.0 & 20.0 & 25.5 & 29.0 & 36.0 \\
\hline 25.0 a 29.9 & 6.5 & 7.0 & 8.0 & 10.0 & 14.0 & 23.0 & 29.0 & 33.0 & 38.5 \\
\hline 30.0 a 34.9 & 6.5 & 7.5 & 8.5 & 10.5 & 16.0 & 26.5 & 32.5 & 37.0 & 43.0 \\
\hline 35.0 a 39.9 & 7.0 & 8.0 & 9.0 & 11.0 & 18.0 & 28.5 & 34.0 & 36.5 & 43.0 \\
\hline 40.0 a 44.9 & 6.5 & 8.0 & 9.0 & 11.5 & 19.0 & 28.5 & 34.0 & 37.0 & 42.0 \\
\hline 45.0 a 49.9 & 7.0 & 8.5 & 10.0 & 12.5 & 20.0 & 29.5 & 34.0 & 37.5 & 43.5 \\
\hline 50.0 a 54.9 & 7.0 & 9.0 & 11.0 & 14.0 & 21.9 & 30.0 & 35.0 & 39.0 & 43.5 \\
\hline 55.0 a 59.9 & 7.0 & 9.0 & 11.0 & 13.5 & 22.0 & 31.0 & 35.0 & 38.0 & 45.0 \\
\hline
\end{tabular}


Distribución de la grasa y riesgo metabólico

\begin{tabular}{|c|c|c|}
\hline $\begin{array}{l}\text { El índice } \\
\text { cintura /cadera }\end{array}$ & ICC $=\frac{\text { Perímetro de la cintura }}{\text { Perímetro de la cadera }}$ & $\begin{array}{l}\text { Mujeres: no debe ser } \\
\text { superior a } 0,80 \\
\text { Varones: no debe ser } \\
\text { superior a 1,0 }\end{array}$ \\
\hline CON ESTE ÍNDICE IDENTIFICAMOS EL TIPO DE OBESIDAD: \\
\hline Hombres & Mujeres & Tipo de obesidad \\
\hline$>=10$ & $>=0.8$ & Androide \\
\hline$<1.0$ & $<0.8$ & Ginoide \\
\hline
\end{tabular}

\section{Circunferencia abdominal}

El tamaño de la circunferencia abdominal también se usa en la actualidad para el diagnóstico de obesidad abdominal.

Mujeres $>80 \mathrm{~cm}$ Obesidad abdominal.

Hombres $>90 \mathrm{~cm}$ Obesidad abdominal.

Puntos de corte para riesgo metabólico

\begin{tabular}{|c|c|c|c|}
\hline \multirow{2}{*}{ Sexo } & \multicolumn{3}{|c|}{ Riesgo } \\
\cline { 2 - 4 } & Bajo & Alto & Muy alto \\
\hline Hombre & $<94 \mathrm{~cm}$ & $\geq 94 \mathrm{~cm}$ & $\geq 102 \mathrm{~cm}$ \\
\hline Mujer & $<80 \mathrm{~cm}$ & $\geq 80 \mathrm{~cm}$ & $\geq 88 \mathrm{~cm}$ \\
\hline
\end{tabular}

Tomado de: Seo DC, ChoeS, Torabi MR. Is waist circumference $\geq 102 / 88 \mathrm{~cm}$ better than body mass index $\geq 30$ to predict hypertension and diabetes development regardless of gender, age group, and race/ethnicity? Metaanalysis. Prev Med. 2017 Apr;97:100-108. 


\section{EVALUACIÓN EN ADULTOS DELGADOS}

\section{Indicadores:}

- IMC

- Perímetro braquial- circunferencia del brazo

- Pliegue tricipital

\section{Perímetro braquial- circunferencia del brazo}

Se ha reconocido como una medida útil para detectar a nivel de campo la desnutrición proteica energética aguda y crónica.

Para calcular la cantidad de masa muscular en las extremidades, se requiere la medición del perímetro braquial (en su punto medio,) que se evaluará así como el pliegue cutáneo correspondiente a la extremidad seleccionada, utilizando la fórmula general:

Área muscular $=($ circunferencia $-\pi \times$ pliegue cutáneo $) 2 / 4 \pi$

Fórmula de Heymsfield

$$
\begin{gathered}
\mathrm{cAMB}=(\mathrm{cm})^{2} \text { mujeres }=\frac{[\mathrm{CB}-(\pi \times \mathrm{PCT})]^{2}}{4 \pi}-6.5 \\
\mathrm{cAMB}=(\mathrm{cm})^{2} \text { hombres }=\frac{[\mathrm{CB}-(\pi \times \mathrm{PCT})]^{2}}{4 \pi}-10.0
\end{gathered}
$$

\section{Instrumentos de Valoración}

\section{Malnutrition universal screening tool (MUST)}

(Instrumento universal para el cribado de la malnutrición)

El MUST es un instrumento de cribado de cinco pasos, diseñado para identificar a adultos malnutridos, con riesgo de malnutrición (desnutrición) u obesos. Incluye además unas directrices de tratamiento, que pueden emplearse para desarrollar un plan de cuidados. Contiene cinco pasos que deben seguirse para el cribado y el tratamiento. 


\section{Los 5 pasos del MUST :}

- Paso 1. mida la estatura y el peso, y calcule la puntuación del IMC utilizando la tabla adjunta. Si no puede obtener la estatura ni el peso, utilice los procedimientos alternativos.

- Paso 2. anote el porcentaje de pérdida de peso involuntaria y la puntuación con ayuda de las tablas adjuntas.

- Paso 3. determine el efecto y la puntuación de las enfermedades agudas.

- Paso 4. sume las puntuaciones de los pasos 1, 2 y 3 para obtener el riesgo global de malnutrición.

- Paso 5. utilice las directrices de tratamiento y/o las normas locales para desarrollar un plan de cuidados.

Paso 1 Puntuación del IMC $\quad+\quad \begin{aligned} & \text { Puntuación de la } \\ & \text { pérdida de peso }\end{aligned}$
Paso 3

Puntuación del efecto de las enfermedades agudas

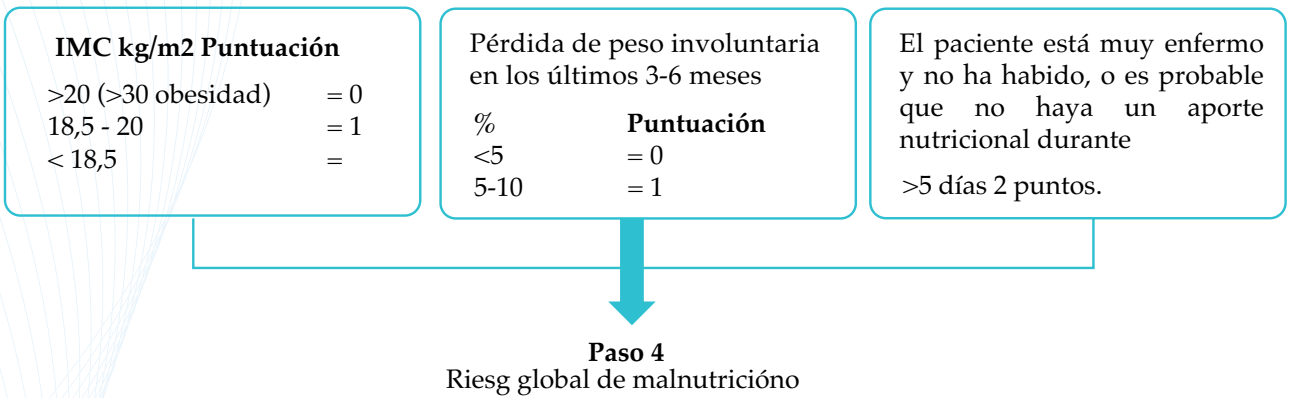

Sume las puntuaciones para calcular el riesgo global de malnutrición.

0 puntos: riesgo bajo; 1 punto: riesgo intermedio; 2 o más puntos: riesgo alto.

Paso 5

Directrices del tratamiento 


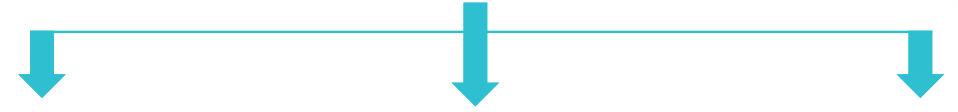

Paso 5

Directrices del tratamiento

\section{Riesgo bajo}

Asistencia clínica habitual

Repetir el cribado.

Hospital: todas las semanas.

Residencias: todos los meses

Comunidad: todos los años en

grupos especiales, p. ej., mayores de 75 años.

\begin{tabular}{l}
\multicolumn{1}{c|}{$\begin{array}{c}\text { 1 Riesgo intermedio } \\
\text { Observación }\end{array}$} \\
$\begin{array}{l}\text { Documentar el aporte dietético } \\
\text { durante } 3 \text { días. }\end{array}$ \\
$\begin{array}{l}\text { Si el aporte es suficiente: escaso } \\
\text { interés clínico; repetir el cribado. }\end{array}$ \\
Hospital: todas las semanas. \\
Residencias: como mínimo todos \\
los meses. \\
Comunidad: como mínimo cada \\
2-3 meses. \\
Si el aporte es insuficiente: \\
interés clínico; seguir las normas \\
locales, fijar objetivos, mejorar y \\
aumentar el aporte nutricional \\
total, controlar y revisar periódica- \\
mente el plan de cuidados.
\end{tabular}

2 o más, riesgo alto
Tratamiento
Derivar a un dietista o a un equipo
de apoyo nutricional, o aplicar las
normas locales.
Fijar objetivos, mejorar y
aumentar el aporte nutricional
total.
Controlar y revisar el plan de
cuidados.
Hospital: todas las semanas.
Residencias: todos los meses.
Comunidad: todos los meses.
*Salvo que sea perjudicial o no se
espere ningún beneficio del apoyo
nutricional, p. ej., en caso de
muerte inminente.

\section{Evaluación global subjetiva (EGS) y riesgo nutricional.}

La EGS constituye una propuesta metodológica sencilla y confiable que parte de la evaluación clínica del sujeto, para determinar si podría presentar desnutrición.

No implica llevar a cabo mediciones y depende de la capacidad del observador para definir la condición del sujeto. Por tanto, el examinador evalúa de manera subjetiva (sin un esquema de puntuación numérica) y clasifica al paciente en una de las categorías.
A. Bien nutrido.
B. Desnutrición moderada.
C. Desnutrición grave. 


\section{HISTORIA}

1. Cambios en el peso

Peso máximo Peso hace 6 meses

Peso actual Pérdida de peso en los últimos 6 meses Porcentaje de pérdida de peso en los últimos 6 meses

Cambios en las últimas 2 semanas: Aumento Sin cambio Disminución

2. Consumo de alimentos (comparado con el habitual) Sin cambio Cambio Duración semanas

\begin{tabular}{l} 
Tipo:__ Aumento \\
\hline Dieta sólida insuficiente \\
\hline Dieta líquida \\
\hline IV o líquidos hipoenergéticos \\
Ayunos
\end{tabular}

3. Síntomas gastrointestinales (duración: $>2$ semanas ) Ninguno Náusea Anorexia Vómito Diarrea

4. Capacidad funcional Sin disfunción Disfunción Duración: semanas Tipo: trabajo ineficiente ambulatorio encamado

EXAMEN FÍSICO (Especificar para cada rasgo: $0=$ normal: $1+=$ ligero, $2+=$ moderado; $3=$ grave )

Pérdida de masa grasa subcutánea (hombros, tríceps, pecho, manos)

Depleción muscular (cuádriceps, músculos deltoides).

Edema de tobillo.

Ascitis

VALORACIÓN DE LA EVALUACIÓN GLOBAL SUBJETIVA (SELECCIONE UNO) $A=$ Bien nutrido

$\mathrm{B}=$ Desnutrición moderada (o sospecha de desnutrición) $\mathrm{C}=$ Desnutrición grave

Adaptado de: Detsky AS, McLaughlin JR, Baker JP, Jhonston N, Whittaker S, Mandelson RA, Jeejeebhoy KN. 1987. What is a Subjective Global Assessment of nutritional status? Journal of Parenteral and Enteral Nutrition 11:8-13. Detsky AS, Smalley PS,Change J. Is this patient malnourished? Journal of the American Medical Association, 1994;271:54-58. 


\section{VALORACIÓN NUTRICIONAL DEL ADULTO MAYOR}

La nutrición juega un papel muy importante en el proceso de envejecimiento, a través de la modulación de cambios en diferentes órganos y funciones del organismo. De allí, la importancia de evaluar el estado nutricional en este grupo.

MEDICIONES ANTROPOMÉTRICAS PARA ESTIMACIÓN DE LA TALLA Y EL PESO EN CASOS ESPECIALES

\section{TALLA}

Cálculo o estimación de la talla: estimación de la talla en personas que no pueden estar en posición vertical: acostadas o que tienen deformidades esqueléticas.

Brazada completa
$\begin{aligned} & \text { Tomar la longitud } \\ & \text { de ambos brazos } \\ & \text { extendidos } \\ & \text { la altura de los } \\ & \text { hombros. }\end{aligned}$
$\begin{aligned} & \text { Estimación de la } \\ & \text { talla por medición } \\ & \text { de la distancia del } \\ & \text { punto medio del } \\ & \text { esternón a la punta } \\ & \text { del dedo medio. }\end{aligned}$
Mujeres: $1,35 \times$ medida de $\mathrm{MB}(\mathrm{cm})+60,1$
Talla (cm) $=63.525$ - $3.237(\mathrm{~S})-0.06904$ (edad) + 1.293 (media brazada)
Dónde: $\mathrm{S}=$ sexo (1 = masculino y $2=$ femenino), edad en años, media
brazada en cm.




\section{CÁLCULO DE LA TALLA: ALTURA TALÓN RODILLA}

Fórmula para calcular la estatura (Chumlea, et al, 1998)

Hombre $=78.31+(1.94 \times$ ATR $)-(0.14 \times$ edad $)$

Mujer $=82.81+(1.85 \times$ ATR $)-(0.21 \times$ edad $)$

$\mathrm{ATR}=$ altura de la rodilla

Mujeres: Talla en $\mathbf{c m}=84.88-(0.24+$ edad $)+(1.83+$ altura de la rodilla en $\mathrm{cm}$ )

Varones: Talla en $\mathbf{c m}=64.19-(0.04+$ edad $)+(2.02+$ altura de la rodilla en $\mathrm{cm}$ ) 


\begin{tabular}{|c|c|c|c|c|c|c|c|c|c|c|c|c|c|c|}
\hline$\stackrel{\infty}{-}$ & $\stackrel{\infty}{\infty}$ & $\begin{array}{l}0 \\
\infty 0^{-} \\
10\end{array}$ & $\begin{array}{l}0 \\
\cong\end{array}$ & $\stackrel{\infty}{\curvearrowright}$ & $\stackrel{\hat{\sigma}}{-}$ & $\underset{i}{\mathbb{H}}$ & $\begin{array}{l}\text { Ln } \\
0 \\
10\end{array}$ & 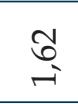 & 요 & 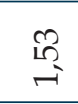 & $\stackrel{\infty}{+}$ & $\stackrel{\circ}{\stackrel{一}{*}}$ & $\underset{+}{\infty}$ & $\underset{+}{+}$ \\
\hline 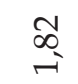 & $\stackrel{\infty}{-\infty}$ & $\begin{array}{l}10 \\
\infty 0^{\circ} \\
10\end{array}$ & $\widehat{尺}$ & $\stackrel{+}{\stackrel{+}{F}}$ & 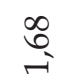 & $\underset{\sim}{10}$ & $\frac{0}{n}$ & $\stackrel{8}{\rightarrow}$ & $\underset{\overbrace{}}{\stackrel{8}{6}}$ & 莫 & 守 & $\begin{array}{l}10 \\
\mathscr{q}\end{array}$ & 孛 & $\stackrel{\text { L }}{\rightarrow}$ \\
\hline$\stackrel{\infty}{\infty}$ & 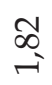 & $\begin{array}{l}\circ \\
\text { ळे } \\
\text { हn }\end{array}$ & $\stackrel{\infty}{\stackrel{\infty}{\approx}}$ & $\stackrel{\mathscr{L}}{\stackrel{2}{\digamma}}$ & $\underset{\mathfrak{o}}{\stackrel{0}{-}}$ & $\underset{\sim}{\stackrel{0}{\circ}}$ & $\stackrel{n}{n}$ & $\underset{+}{\stackrel{H}{-}}$ & $\underset{\sigma}{-}$ & 占 & $\stackrel{\text { மூ }}{\sim}$ & $\stackrel{\circ}{\stackrel{+}{f}}$ & ڤి & to \\
\hline$\underset{+\infty}{\stackrel{\infty}{-}}$ & $\stackrel{\infty}{\infty}$ & $\begin{array}{l}\text { L2 } \\
\text { જे }\end{array}$ & $\stackrel{\Re}{\curvearrowright}$ & $\stackrel{0}{\stackrel{1}{=}}$ & $\stackrel{尺}{\curvearrowright}$ & $\stackrel{\hat{6}}{-}$ & $\begin{array}{l}\circ \\
\text { N่ } \\
\text { กิ }\end{array}$ & $\stackrel{10}{\stackrel{10}{-}}$ & $\underset{-}{\mathbb{V}}$ & 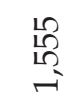 & กี & $\stackrel{n}{f}$ & 로 & $\stackrel{\text { F }}{\rightarrow}$ \\
\hline$\stackrel{10}{\infty}$ & $\stackrel{+\infty}{\text { \& }}$ & $\begin{array}{l}\circ \\
\text { ஜे }\end{array}$ & $\stackrel{\infty}{\infty}$ & $\hat{尺}$ & 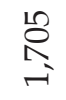 & $\stackrel{\infty}{\infty}$ & $\begin{array}{l}\text { ñ } \\
\text { กิ }\end{array}$ & 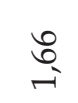 & 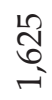 & 占 & 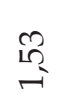 & $\begin{array}{l}0 \\
10 \\
\stackrel{4}{7}\end{array}$ & กิ & $\stackrel{\infty}{\stackrel{\infty}{+}}$ \\
\hline 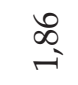 & $\stackrel{\llcorner 0}{\infty}$ & 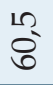 & $\stackrel{\infty}{-\infty}$ & $\stackrel{\infty}{\stackrel{\infty}{\rightleftharpoons}}$ & $\widetilde{\curvearrowright}$ & 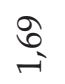 & $\begin{array}{l}0 \\
\tilde{N}^{-}\end{array}$ & $\stackrel{\hat{\sigma}}{\rightarrow}$ & 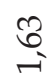 & مि & 苞 & $\begin{array}{l}10 \\
10\end{array}$ & $\stackrel{\infty}{n}$ & 孛 \\
\hline $\begin{array}{l}10 \\
\infty \\
\infty \\
-\end{array}$ & $\stackrel{\infty}{\infty}$ & $\frac{0}{6}$ & 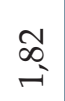 & $\stackrel{\Re}{\widehat{尺}}$ & 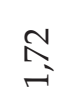 & $\stackrel{尺}{\stackrel{1}{F}}$ & $\begin{array}{l}n \\
\text { ñ } \\
\text { กी }\end{array}$ & 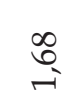 & $\underset{-}{+}$ & $\begin{array}{l}\infty \\
\stackrel{\infty}{2} \\
\rightleftharpoons\end{array}$ & 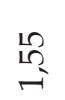 & $\begin{array}{l}0 \\
\text { o' } \\
\text { tr }\end{array}$ & $\stackrel{H}{\stackrel{H}{n}}$ & 옥 \\
\hline$\stackrel{\infty}{\infty}$ & $\stackrel{\infty}{\oplus}$ & in & $\stackrel{\infty}{\infty}$ & $\stackrel{\infty}{\infty}$ & $\stackrel{\mathscr{N}}{\stackrel{N}{\rightleftharpoons}}$ & 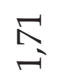 & $\stackrel{\circ}{\stackrel{5}{1}}$ & 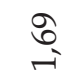 & $\stackrel{10}{6}$ & เి & مٌ & $\begin{array}{l}n \\
\operatorname{lo}^{\circ}\end{array}$ & $\stackrel{102}{\text { Ln }}$ & 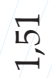 \\
\hline $\begin{array}{l}\infty \\
\infty \\
-\end{array}$ & $\stackrel{\infty}{\infty}$ & $\begin{array}{l}\circ \\
\text { กิ }\end{array}$ & $\underset{-}{\stackrel{+}{\infty}}$ & $\stackrel{\infty}{-}$ & $\stackrel{+}{\stackrel{+}{F}}$ & $\underset{F}{\mathbb{N}}$ & सn & $\stackrel{尺}{\stackrel{2}{r}}$ & $\stackrel{?}{\stackrel{0}{*}}$ & $\stackrel{8}{8}$ & ڤિ & $\stackrel{\circ}{\stackrel{f}{*}}$ & 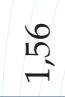 & กิ \\
\hline$\stackrel{\infty}{\stackrel{\infty}{-}}$ & $\stackrel{\infty}{-\infty}$ & ָึ & $\stackrel{10}{\infty}$ & $\underset{-\infty}{\infty}$ & 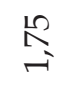 & $\stackrel{\Re}{\cong}$ & $\begin{array}{l}0 \\
10 \\
10\end{array}$ & 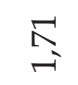 & $\stackrel{\hat{\sigma}}{-}$ & $\stackrel{\overrightarrow{6}}{=}$ & $\begin{array}{l}\infty \\
\stackrel{1}{n} \\
\end{array}$ & $\stackrel{12}{\stackrel{\circ}{f}}$ & م̂م & 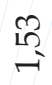 \\
\hline § & 尺) & ণิ & $\begin{array}{l}0 \\
\infty \\
-1\end{array}$ & $\stackrel{\infty}{\infty}$ & $\stackrel{0}{\stackrel{2}{F}}$ & $\stackrel{+}{\stackrel{+}{F}}$ & $\begin{array}{l}10 \\
10 \\
10\end{array}$ & $\stackrel{N}{\sim}$ & $\stackrel{\infty}{\infty} \underset{\sim}{\stackrel{0}{*}}$ & $\underset{\overbrace{}}{\widetilde{C}}$ & है & $\begin{array}{c}\circ \\
\infty \\
\infty \\
+\end{array}$ & $\begin{array}{l}\infty \\
\stackrel{1}{n} \\
\end{array}$ & 范 \\
\hline శ్ & స్ & $\begin{array}{l}2 \\
\text { ชి }\end{array}$ & $\stackrel{N}{\infty}$ & $\begin{array}{l}\infty \\
\infty \\
\infty \\
\stackrel{\infty}{=}\end{array}$ & $\stackrel{\curvearrowright}{\curvearrowright}$ & $\stackrel{0}{\stackrel{1}{F}}$ & $\begin{array}{l}\text { જे } \\
\text { மొ }\end{array}$ & $\stackrel{\Re}{\stackrel{\Omega}{\sim}}$ & $\stackrel{\hat{\sigma}}{-}$ & 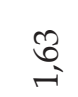 & $\underset{i}{\stackrel{8}{1}}$ & $\begin{array}{l}12 \\
\infty 0^{\circ}\end{array}$ & $\begin{array}{l}10 \\
\infty \\
19 \\
-\end{array}$ & $\stackrel{10}{\stackrel{L}{L}}$ \\
\hline م્ & స్ & $\stackrel{\circ}{\stackrel{+}{0}}$ & 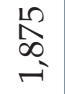 & $\stackrel{+}{+\infty}$ & $\stackrel{\infty}{\stackrel{\infty}{\digamma}}$ & $\stackrel{\widehat{~}}{\stackrel{-}{F}}$ & $\begin{array}{l}12 \\
\text { है } \\
10\end{array}$ & $\stackrel{\stackrel{2}{\curvearrowright}}{\stackrel{2}{\rightleftharpoons}}$ & $\stackrel{R}{r}$ & ț & $\stackrel{\vec{b}}{-}$ & $\begin{array}{l}0 \\
\text { ने }\end{array}$ & ڤે & டొ \\
\hline$\stackrel{\Omega}{\sigma}$ & $\stackrel{\Omega}{\sigma}$ & $\stackrel{n}{\sharp}$ & $\stackrel{\infty}{\infty}$ & $\stackrel{\infty}{\infty}$ & શ્ & $\stackrel{\infty}{\underset{1}{\sim}}$ & 옹 & $\stackrel{+}{\stackrel{H}{F}}$ & $\widetilde{\curvearrowright}$ & $\stackrel{10}{6}$ & $\underset{్}{\mathfrak{G}}$ & مू & $\underset{\overbrace{}}{\stackrel{8}{i}}$ & مि \\
\hline 今્ & హ్ & $\begin{array}{l}0 \\
10 \\
0\end{array}$ & $\stackrel{\infty}{\infty}$ & $\stackrel{\infty}{\infty}$ & $\stackrel{\infty}{\infty}$ & $\stackrel{\Re}{\curvearrowright}$ & 贻 & $\stackrel{10}{\stackrel{10}{\overbrace{}}}$ & $\stackrel{N}{\approx}$ & 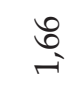 & $\underset{\overbrace{}}{\mathscr{b}}$ & $\begin{array}{l}0 \\
\text { ○े } \\
\text { ட) }\end{array}$ & $\stackrel{\sigma ్}{-}$ & $\stackrel{\infty}{\stackrel{\infty}{\llcorner}}$ \\
\hline 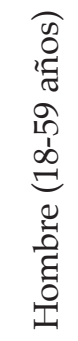 & 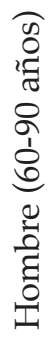 & 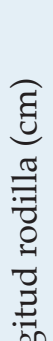 & 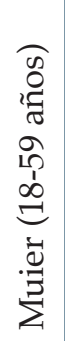 & 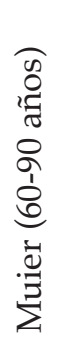 & 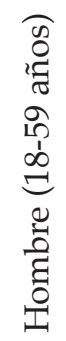 & 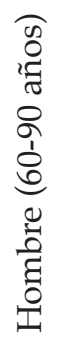 & 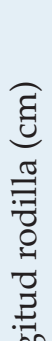 & 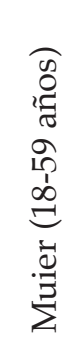 & 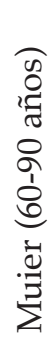 & 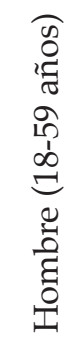 & 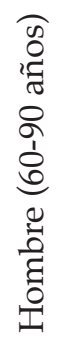 & 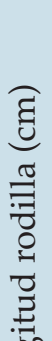 & 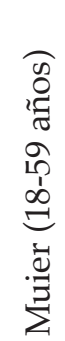 & 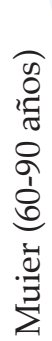 \\
\hline Talla & & క్ర & Tall & (m) & Tall & (m) & కే & Talla & & Tall & & 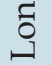 & Tall & \\
\hline
\end{tabular}




\section{PESO}

Ecuaciones para estimar el peso en varones y mujeres de entre 65 y 90 años de edad.

\begin{tabular}{|l|l|}
\hline Varones & $(1.73 \times \mathrm{CMB})+(0.98 \times \mathrm{CP})+(0.37 \times \mathrm{PCS})+(1.16 \times \mathrm{AR})-81.69$ \\
\hline Mujeres & $(0.98 \times \mathrm{CMB})+(1.27 \times \mathrm{CP})+(0.4 \times \mathrm{PCS})+(0.87 \times \mathrm{AR})-62.35$
\end{tabular}

CMB, circunferencia media del brazo $(\mathrm{cm})$.

$\mathrm{CP}$, circunferencia de la pantorrilla $(\mathrm{cm})$.

PCS, pliegue cutaneo subescapular (mm).

AR, altura de la rodilla $(\mathrm{cm})$.

Tomado de: Chumlea WC, Shumei, Roche, et al. Prediction of body weight for the nonambulatory elderly from anthropometry. JAm Diet Assoc, 1988;88:564-568.15.

\section{Índice de masa corporal}

Índice de masa corporal normal según lo establecido en el NHANES III (tercer estudio para el examen de salud y nutrición)

Edad

Años

$50-59$

$60-69$

$70-79$

80 y más

$22,4-27$
Índice de masa corporal normal $\left(\mathrm{kg} / \mathrm{m}^{2}\right)$

hombres

mujeres

23.6-32.1

$23.5-30,8$

22.6-29.9

$21,7-28,4$

Clasificación de la valoración nutricional de las personas adultas mayores según índice de masa corporal (IMC).

\begin{tabular}{|l|l|}
\hline \multicolumn{1}{|c|}{ CLASIFICACIÓN } & \multicolumn{1}{c|}{ IMC } \\
\hline Delgadez & $\leq 23.0$ \\
Normal & $>23 \mathrm{a}<28$ \\
Sobrepeso & $\geq 28 \mathrm{a}<32$ \\
Obesidad & $\geq 32$ \\
\hline
\end{tabular}

Tomado de: Organización Panamericana de la Salud (OPS). Guía clínica para atención primaria a las personas adultas mayores. Módulo 5. Valoración nutricional del adulto mayor. Washington, DC 2002. 


\section{Identificación de los trastornos de la composición corporal}

\begin{tabular}{|c|c|}
\hline \multicolumn{2}{|l|}{ EMACIACIÓN } \\
\hline \multicolumn{2}{|l|}{ Reconocimiento: } \\
\hline \multicolumn{2}{|l|}{ Métodos Clínicos } \\
\hline \multicolumn{2}{|l|}{ Métodos Antropométricos } \\
\hline & Antropometría. \\
\hline Valoración clínica. & Toma de peso y talla \\
\hline Inspección general del & Cálculo del IMC. \\
\hline Apariencia física. & $\begin{array}{l}\text { Toma de la circunferencia del brazo } \\
\text { (CB). }\end{array}$ \\
\hline Inspección del volumen de los & Toma del pliegue tricipital (PCT). \\
\hline & $\begin{array}{l}\text { Cálculo de la área muscular del brazo } \\
\text { (AMB) }\end{array}$ \\
\hline Criterios diagnósticos & IMC $<=23$ \\
\hline Pérdida de peso leve & PCT $<4,5 \mathrm{~mm}$ hombres \\
\hline Disminución del IMC & $<12 \mathrm{~mm}$ mujeres \\
\hline Disminución del PCT & AMB $>31,4$ hombres \\
\hline $\begin{array}{l}\text { Preservación de la masa } \\
\text { muscular. }\end{array}$ & $>21,9$ mujeres \\
\hline
\end{tabular}

\section{SARCOPENIA}

Reconocimiento:

Métodos clínicos

Métodos antropométricos 


\begin{tabular}{|c|c|}
\hline $\begin{array}{l}\text { Valoración clínica. } \\
\text { Inspección general del paciente. } \\
\text { Apariencia física }\end{array}$ & $\begin{array}{l}\text { Antropometría. } \\
\text { Toma del peso y talla } \\
\text { Cálculo del IMC. } \\
\text { Medición de la circunferencia } \\
\text { del brazo (CB) y de la } \\
\text { circunferencia de la pantorrilla } \\
\text { (CP). }\end{array}$ \\
\hline $\begin{array}{l}\text { Inspección del volumen y forma de } \\
\text { las masas musculares. }\end{array}$ & $\begin{array}{l}\text { Medición del pliegue cutáneo } \\
\text { tricipital. } \\
\text { Cálculo del área muscular del } \\
\text { brazo (AMB). }\end{array}$ \\
\hline $\begin{array}{l}\text { Criterios diagnósticos } \\
\text { IMC normal o levemente disminuido. } \\
\text { Disminución del CB y/o AMB y/o } \\
\text { CP } \\
\text { PCT normal o aumentado }\end{array}$ & $\begin{array}{l}\text { Criterios diagnósticos } \\
\text { CB } \quad<25,4 \text { hombres } \\
\quad<24,3 \text { mujeres } \\
\text { AMB } \quad<31,4 \text { hombres } \\
\quad<21,9 \text { mujeres } \\
\quad<31,0 \text { hombres / mujeres } \\
\text { CP } \quad>=4,5 \text { mm hombres } \\
\text { PCT } \quad>12 \text { mm mujeres } \\
\text { Obesidad } \\
\text { Disminución del CB y/o AMB } \\
\text { y/o CP } \\
\text { PCT }>15,0 \text { hombres } \\
\quad>30,0 \text { mujeres }\end{array}$ \\
\hline
\end{tabular}

\section{CAQUEXIA}

Reconocimiento:

Métodos clínicos

Métodos antropométricos 


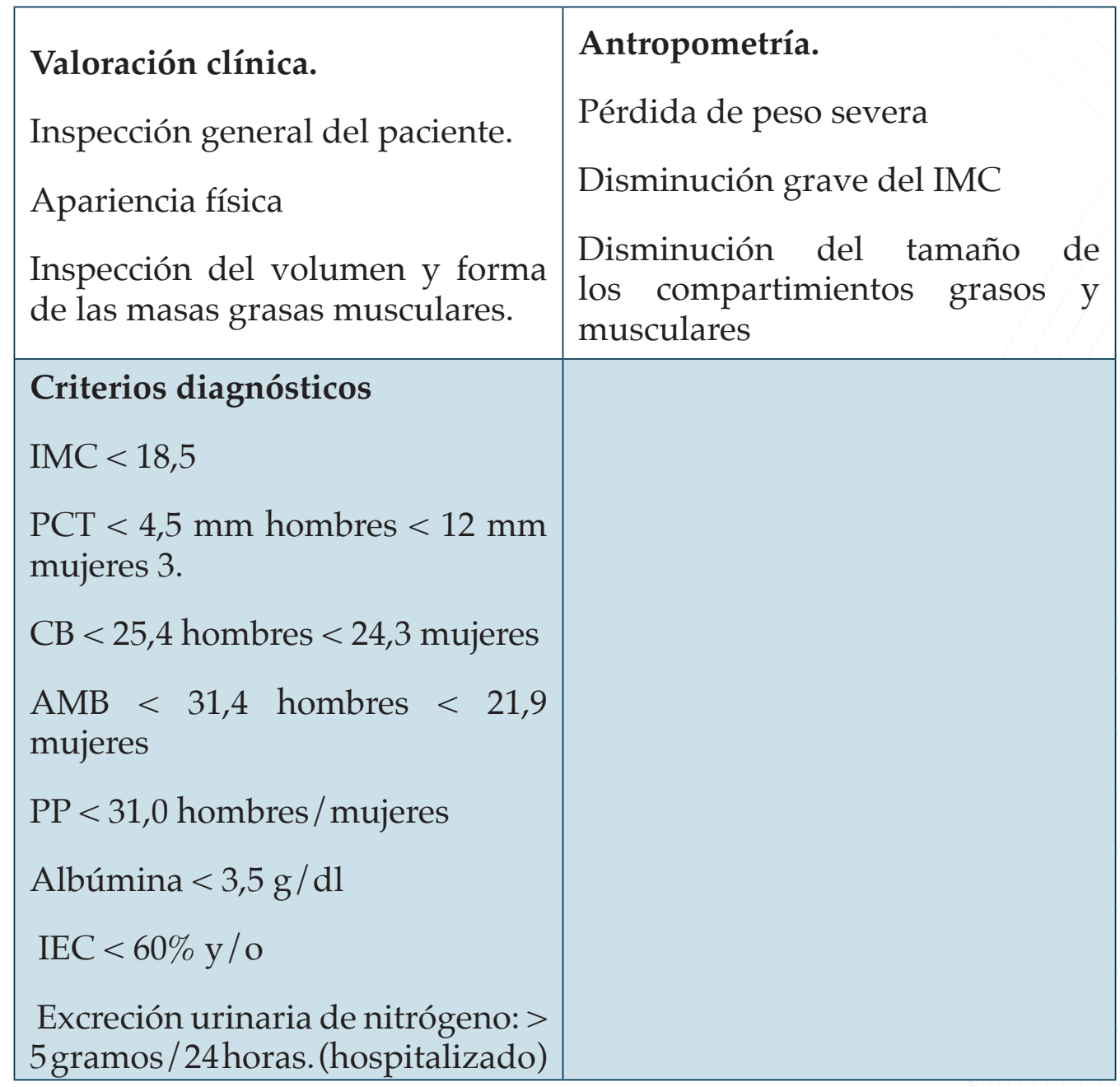

\begin{tabular}{|l|}
\hline \\
Cálculo de la $\mathrm{AMB}$
\end{tabular}


La MNA es una herramienta clínica de evaluación del estado nutricional del adulto mayor. Contiene 2 secciones: pesquisaje y evaluación.

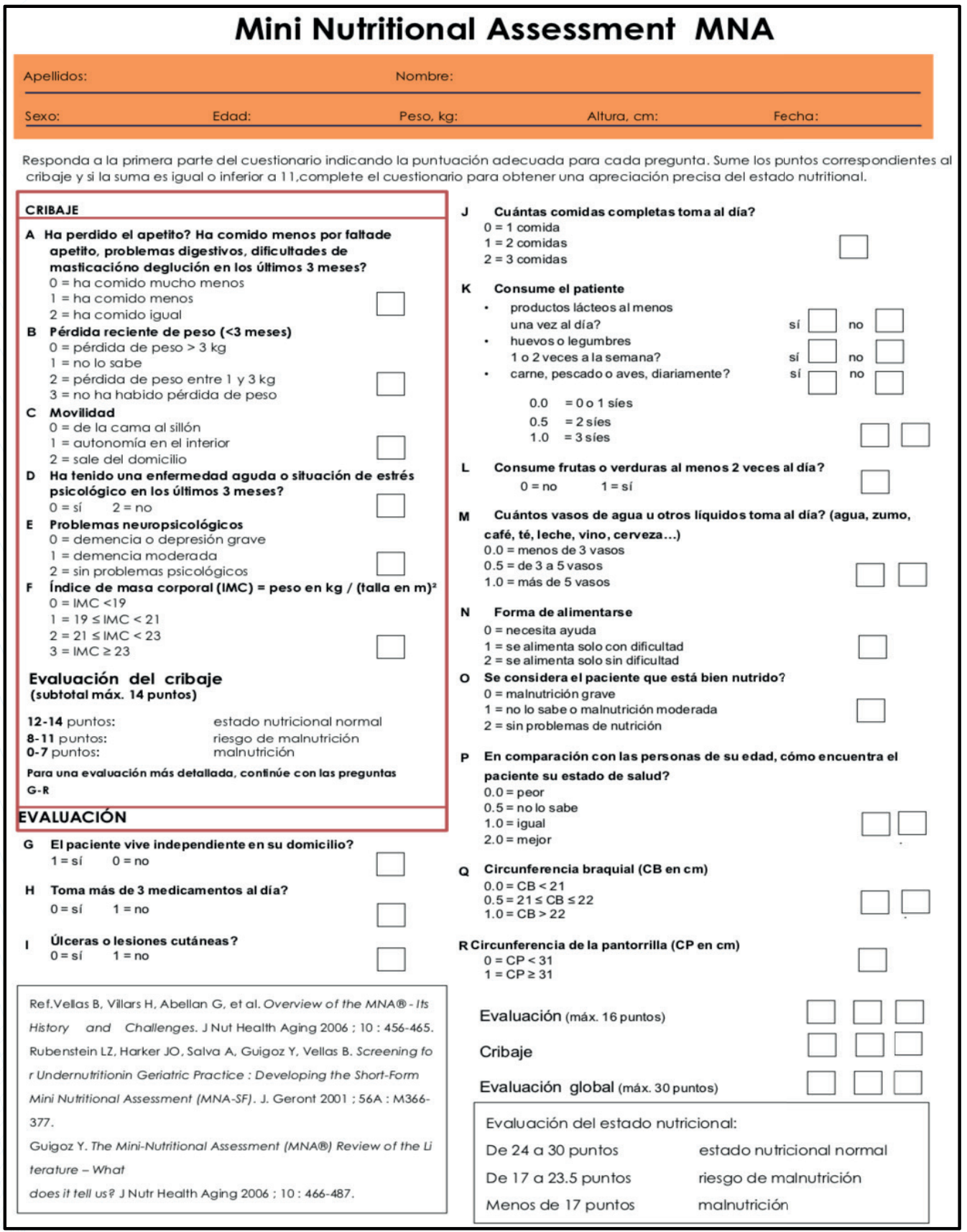




\section{CUESTIONARIO}

\section{CONOZCA SU SALUD NUTRICIONAL}

Se utiliza frecuentemente con personas de 60 años. Fue desarrollado y distribuido por la Nutritional Screening Initiative (NSI).

\section{CONOZCA SU SALUD NUTRICIONAL}

\begin{tabular}{|l|c|c|}
\hline $\begin{array}{l}\text { ¿Ha tenido una enfermedad o afección que le ha hecho } \\
\text { cambiar el tipo y/o cantidad de alimento que come? }\end{array}$ & Sí & No \\
\hline ¿Toma menos de dos comidas al día? & Sí & No \\
\hline ¿Come poca fruta, vegetales o productos lácteos? & Sí & No \\
\hline $\begin{array}{l}\text { ¿Toma más de tres vasos de cerveza, licor o vino, casi a } \\
\text { diario? }\end{array}$ & Sí & No \\
\hline ¿Tiene problemas dentales que le dificultan comer? & Sí & No \\
\hline $\begin{array}{l}\text { ¿No siempre tiene suficiente dinero para comprar la } \\
\text { comida que necesita? }\end{array}$ & Sí No \\
\hline $\begin{array}{l}\text { ¿Come solo, la mayoría de las veces? } \\
\text { ¿Toma a diario tres o más fármacos recetados por su } \\
\text { cuenta? }\end{array}$ & Sí & No \\
\hline $\begin{array}{l}\text { ¿Sin querer, ha perdido o ganado 5kg de peso en los } \\
\text { últimos seis meses? }\end{array}$ & Sí & No \\
\hline $\begin{array}{l}\text { ¿No siempre puedo comprar, cocinar y/o comer por mí } \\
\text { mismo, por problemas físicos? }\end{array}$ & Sí & No \\
\hline
\end{tabular}

\section{PUNTUACIÓN}

\section{SOLO PUNTÚA LA AFIRMACIÓN SÍ}

\begin{tabular}{|l|c|c|}
\hline PUNTUACIÓN SEGÚN RESPUESTA & SÍ & NO \\
\hline $\begin{array}{l}\text { ¿Ha tenido una enfermedad o afección que le ha hecho } \\
\text { cambiar el tipo y/o cantidad de alimento que come? }\end{array}$ & 2 & 0 \\
\hline ¿Toma menos de dos comidas al día? & 3 & 0 \\
\hline \begin{tabular}{l} 
¿Come poca fruta, vegetales o productos lácteos? \\
\hline $\begin{array}{l}\text { ¿Toma más de tres vasos de cerveza, licor o vino, casi a } \\
\text { diario? }\end{array}$
\end{tabular} & 2 & 0 \\
\hline
\end{tabular}




\begin{tabular}{|l|l|l|}
\hline ¿Tiene problemas dentales que le dificultan comer? & 2 & 0 \\
\hline $\begin{array}{l}\text { ¿No siempre tiene suficiente dinero para comprar la } \\
\text { comida que necesita? }\end{array}$ & 4 & 0 \\
\hline ¿Come solo, la mayoría de las veces? & 1 & 0 \\
\hline $\begin{array}{l}\text { ¿Toma a diario tres o más fármacos recetados por su } \\
\text { cuenta? }\end{array}$ & 1 & 0 \\
\hline $\begin{array}{l}\text { ¿Sin querer, ha perdido o ganado 5kg de peso en los } \\
\text { últimos seis meses? }\end{array}$ & 2 & 0 \\
\hline $\begin{array}{l}\text { ¿No siempre puedo comprar, cocinar y/o comer por mí } \\
\text { mismo, por problemas físicos? }\end{array}$ & 2 & 0 \\
\hline Puntuación total & & \\
\hline
\end{tabular}

\begin{tabular}{|c|c|}
\hline \multicolumn{2}{|c|}{ PUNTOS DE CORTE } \\
\hline 0 a 2: Bueno & Reevaluar la puntuación en seis meses. \\
\hline $\begin{array}{c}3 \text { a 5: Riesgo nutricional } \\
\text { moderado }\end{array}$ & $\begin{array}{l}\text { Tomar medidas para mejorar los } \\
\text { hábitos alimentarios y el estilo de vida. } \\
\text { Reevaluar en tres meses. }\end{array}$ \\
\hline $\begin{array}{c}6 \text { o más: Riesgo nutricional } \\
\text { alto }\end{array}$ & $\begin{array}{l}\text { Lleve el cuestionario a su nutricionista } \\
\text { y pida ayuda para mejorar su estado } \\
\text { nutricional. }\end{array}$ \\
\hline
\end{tabular}

\section{Bibliografía}

1. Suverza Fernández, A. El ABCD de la Evaluación del Estado de Nutrición. México:McGraw-Hill Interamericana. 2010.

2. Suverza Fernández, A. El ABCD de la Evaluación del Estado de Nutrición. México:McGraw-Hill Interamericana. 2010.

3. Chumlea WC, Shumei, Roche, et al. Prediction of body weight for the nonambulatory elderly from anthropometry. JAm Diet Assoc, 1988;88:564-568.15.

4. Organización Panamericana de la Salud (OPS). Guía Clínica para Atención Primaria a las personas Adultas Mayores. Módulo 5. Valoración Nutricional del Adulto Mayor. Washington, DC 2002.

5. Elia M. (Chairman and Editor) Guidelines for detection and 
management of malnutrition. Malnutrition Advisory Group (MAG), Standing Committee of BAPEN (ISBN 1899467 459) 2000. Explanatory Booklet A Guide to the 'Malnutrition Universal Screening Tool' ('MUST') for Adults.

6. Seo DC, Choe $S$, Torabi MR. Is waist circumference $\geq 102 / 88 \mathrm{~cm}$ better than body mass index $\geq 30$ to predict hypertension and diabetes development regardless of gender, age group, and race/ethnicity? Meta-analysis. Prev Med. 2017 Apr;97:100-108.

7. Detsky AS, McLaughlin JR, Baker JP, Jhonston N, Whittaker S, Mandelson RA, Jeejeebhoy KN. 1987. What is a Subjective Global Assessment of nutritional status? Journal of Parenteral and Enteral Nutrition 11:8-13. Detsky AS, Smalley PS,Change J. Is this patient malnourished? Journal of the American Medical Association, 1994;271:54-58.

8. Body fat assessment from total body density and its estimation from skinfold thickness: Measurements on 481 men and women aged 16-72 years. British Journal of Nutrition 1974; 32:77-97.

9. NIH. Clinical Guidelines Identification, Evaluation, and Treatment of Overweight and Obesity in Adults. WHO. Obesity: Preventing and managing the global epidemic. 98. NOM-174-SSA1-98. 


\section{APÉNDICE II.}

\section{CÁlCULOS Y ESCALAS EN LA UNIDAD DE CUIDADOS INTENSIVOS}

Dr. Francisco Villacorta.

Médico Especialista en Medicina Crítica y Terapia Intensiva

Los sistemas de scores son herramientas de severidad, gravedad y diagnóstico de la enfermedad, útiles en cualquier nivel hospitalario, que se utilizan para predecir los resultados, generalmente la mortalidad, severidad y la posibilidad diagnóstica de los pacientes en la unidad de cuidados intensivos (UCI).

Tales medidas son útiles para estandarizar la investigación y comparar la calidad de la atención al paciente en la UCI. Los sistemas validados más comúnes de puntuación predictiva y diagnóstico, utilizados en la Unidad de cuidados intensivos se describen a continuación:

\section{ISOGRAVEDAD}

Apache: es uno de los sistemas más frecuentemente utilizados para cuantificar la gravedad de un paciente con independencia del diagnóstico, evaluando los parámetros fisiológicos.

Ayuda en la predicción de mortalidad la duración de la estancia en UCI, además es preciso para diferenciar de quienes sobrevivirán y quienes no sobrevivirán. 


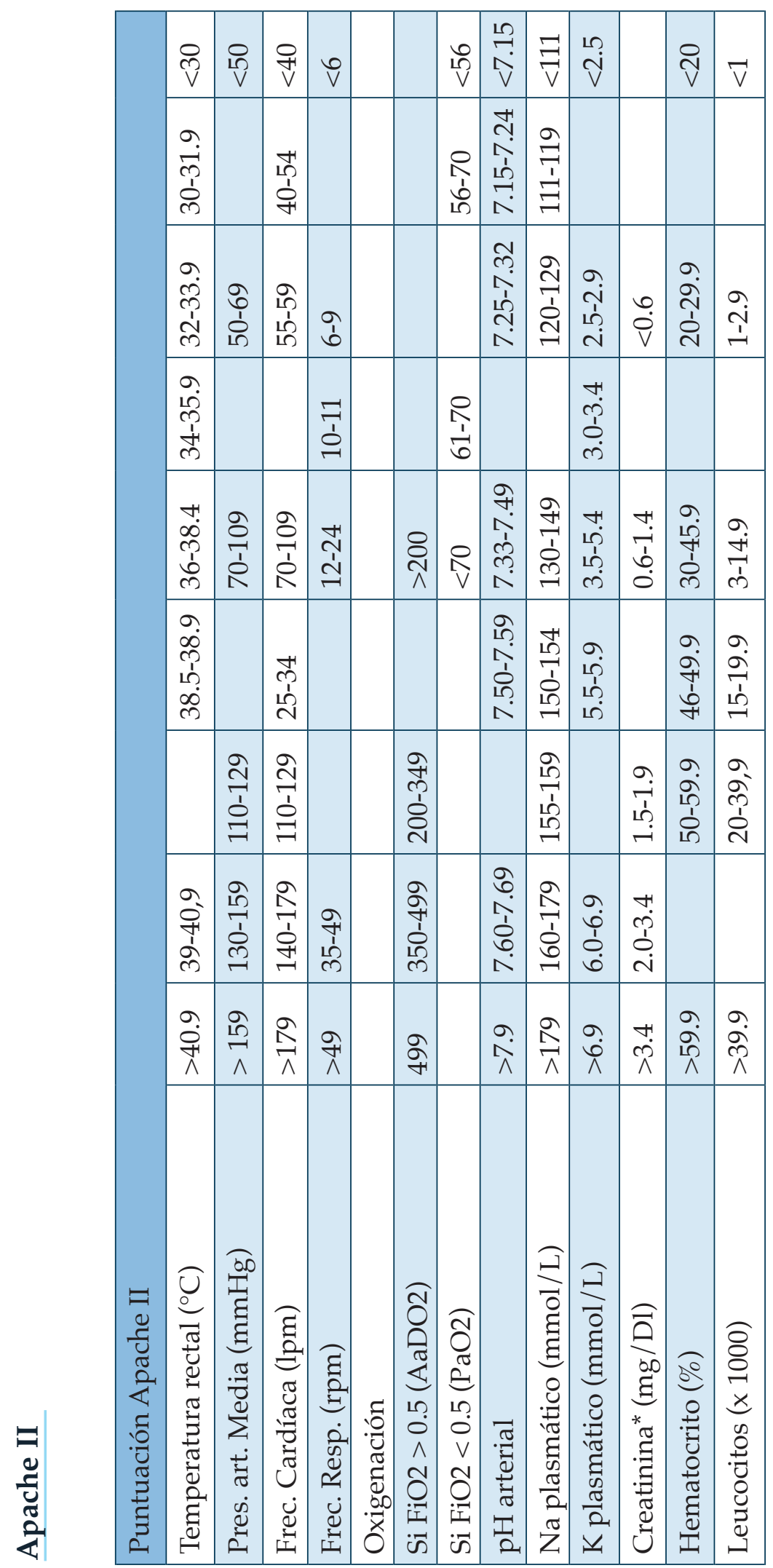




\section{Suma de puntos}

Total, APS

15 GSC

ENFERMEDAD CRÓNICA

\begin{tabular}{|l|c|}
\hline Preoperatorio programado & 2 \\
\hline Preoperatorio urgente o médico & 5 \\
\hline EDAD & 0 \\
\hline$<44$ & 2 \\
\hline $45-64$ & 3 \\
\hline $55-64$ & 5 \\
\hline $65-74$ & 6 \\
\hline$>75$ & \\
\hline
\end{tabular}

\begin{tabular}{|l|l|l|l|}
\hline $\begin{array}{l}\text { Puntos APS } \\
\text { (A) }\end{array}$ & $\begin{array}{l}\text { Puntos GCS } \\
\text { (B) }\end{array}$ & $\begin{array}{l}\text { Puntos edad } \\
\text { (C) }\end{array}$ & $\begin{array}{l}\text { Puntos enf. Previa } \\
\text { (D) }\end{array}$ \\
\hline & & & \\
\hline
\end{tabular}




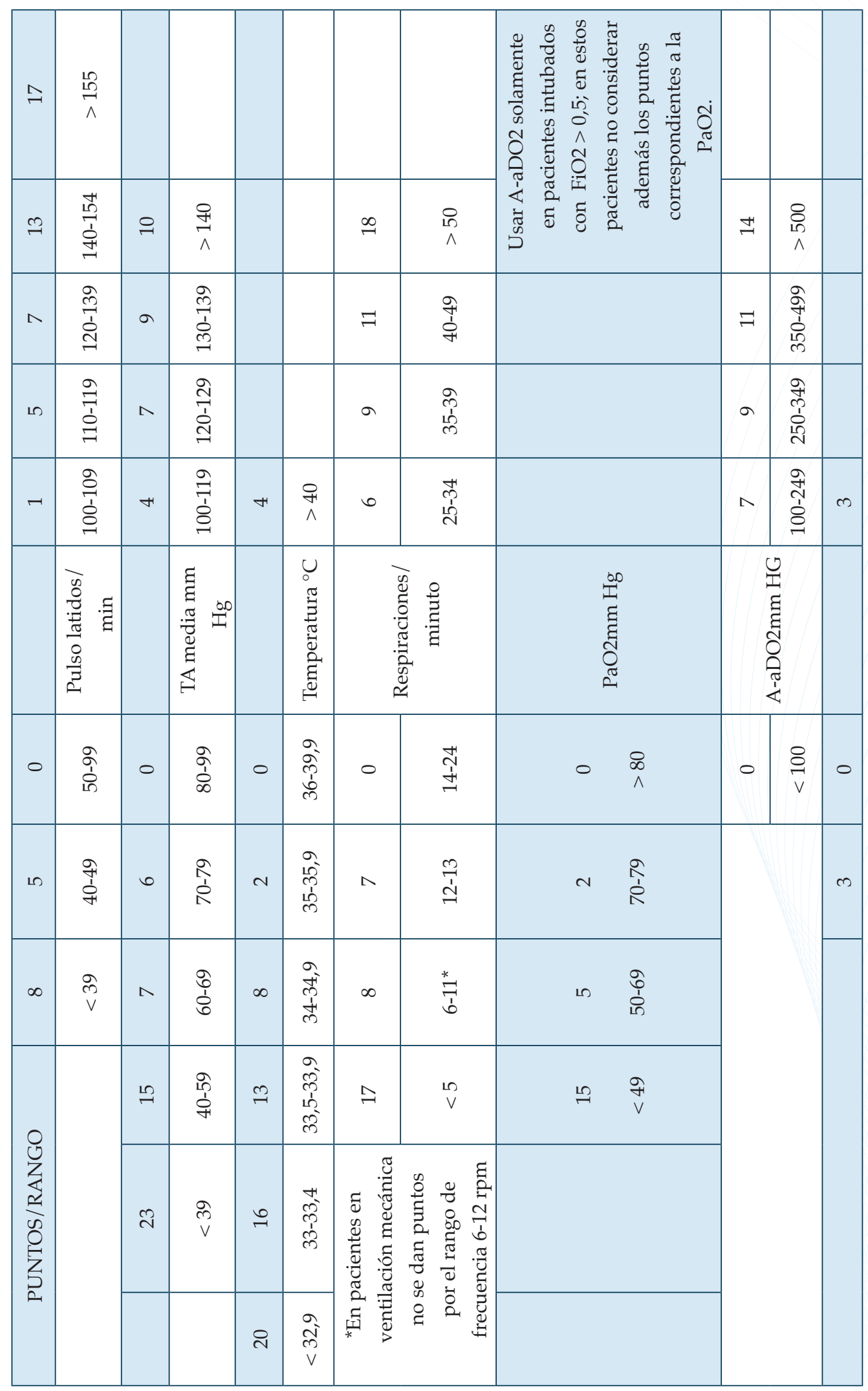




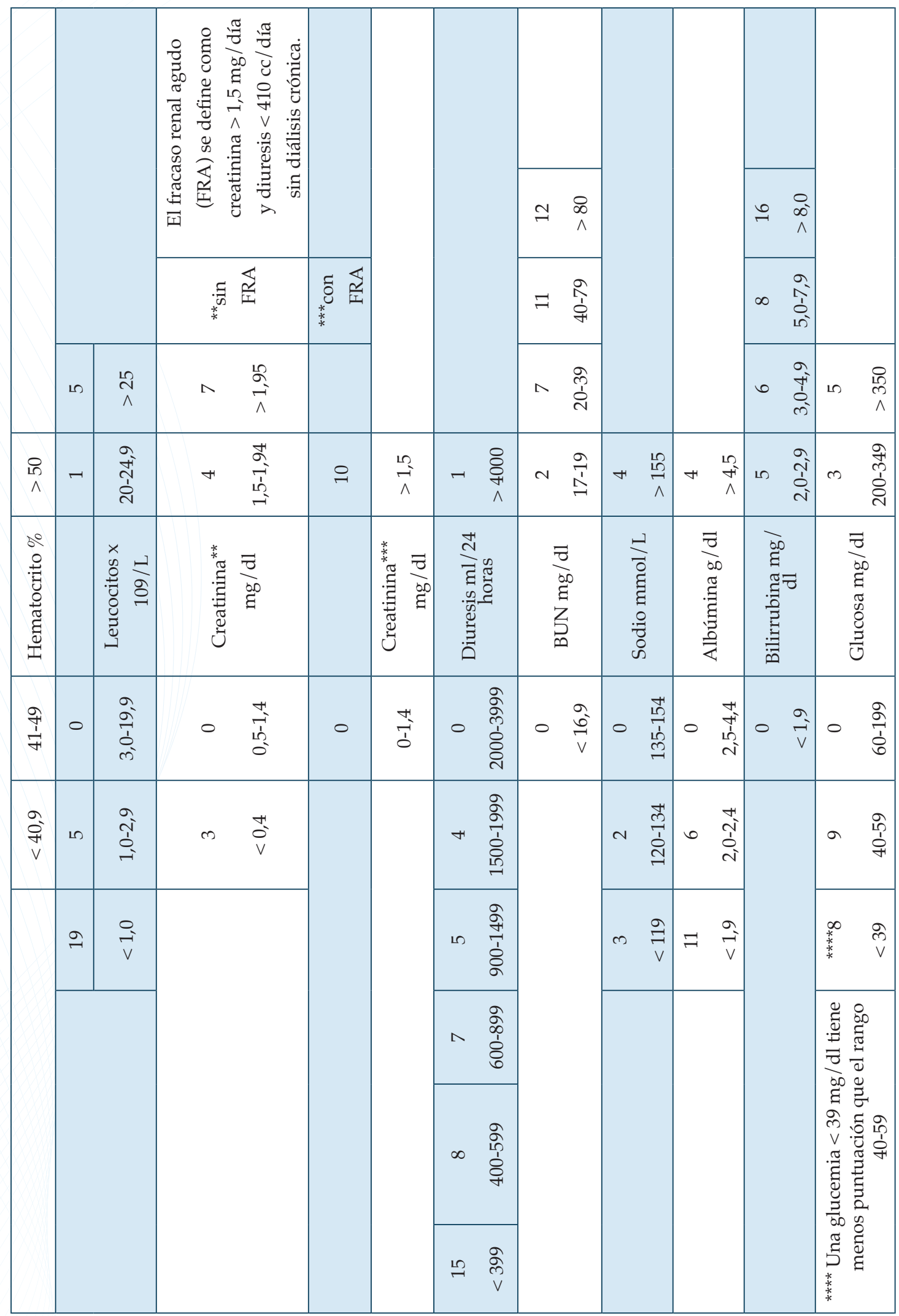




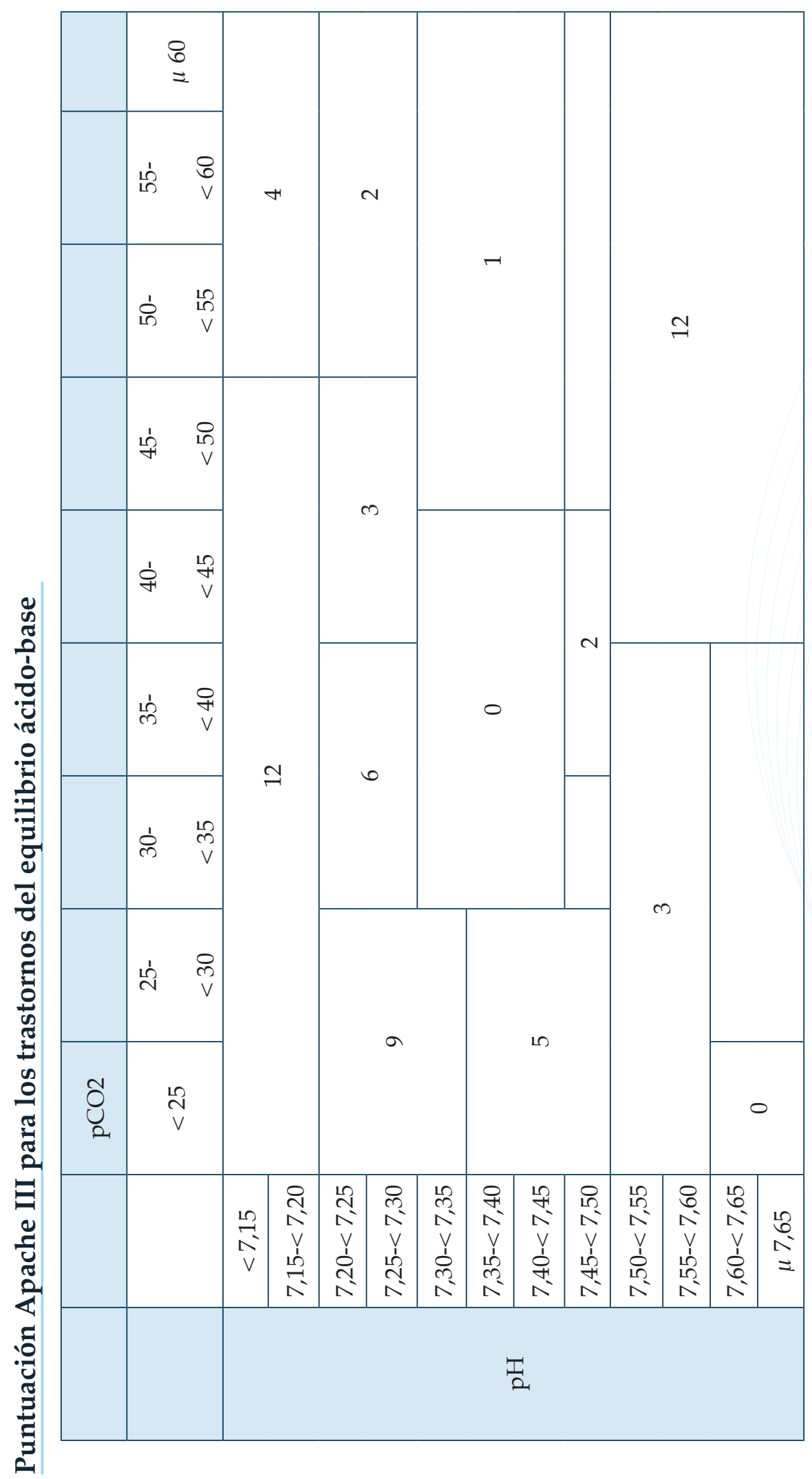




\section{Puntuaciones Apache III para la edad y el estado de salud crónico}

\begin{tabular}{|c|c|}
\hline & PUNTOS \\
\hline \multicolumn{2}{|l|}{ Edad, años } \\
\hline$<44$ & 0 \\
\hline $45-59$ & 5 \\
\hline $60-64$ & 11 \\
\hline $65-69$ & 13 \\
\hline $70-74$ & 16 \\
\hline $75-84$ & 17 \\
\hline$\mu 85$ & 24 \\
\hline \multicolumn{2}{|l|}{ Comorbilidades* } \\
\hline SIDA & 23 \\
\hline Fallo hepático & 16 \\
\hline Linfoma & 13 \\
\hline Carcinoma metastásico & 11 \\
\hline Leucemia/Mieloma múltiple & 10 \\
\hline Inmunosupresión & 10 \\
\hline Cirrosis & 4 \\
\hline *Excluidas en los pacientes de cirugía electiva. & \\
\hline
\end{tabular}

Puntuación Apache III para las alteraciones neurológicas de acuerdo con la presencia o ausencia de apertura de ojos. Las áreas sombreadas o sin puntuación representan combinaciones clínicas inusuales o improbables. La colocación de un paciente en alguna de esas casillas debería hacerse después de una cuidadosa confirmación de los hallazgos clínicos. 


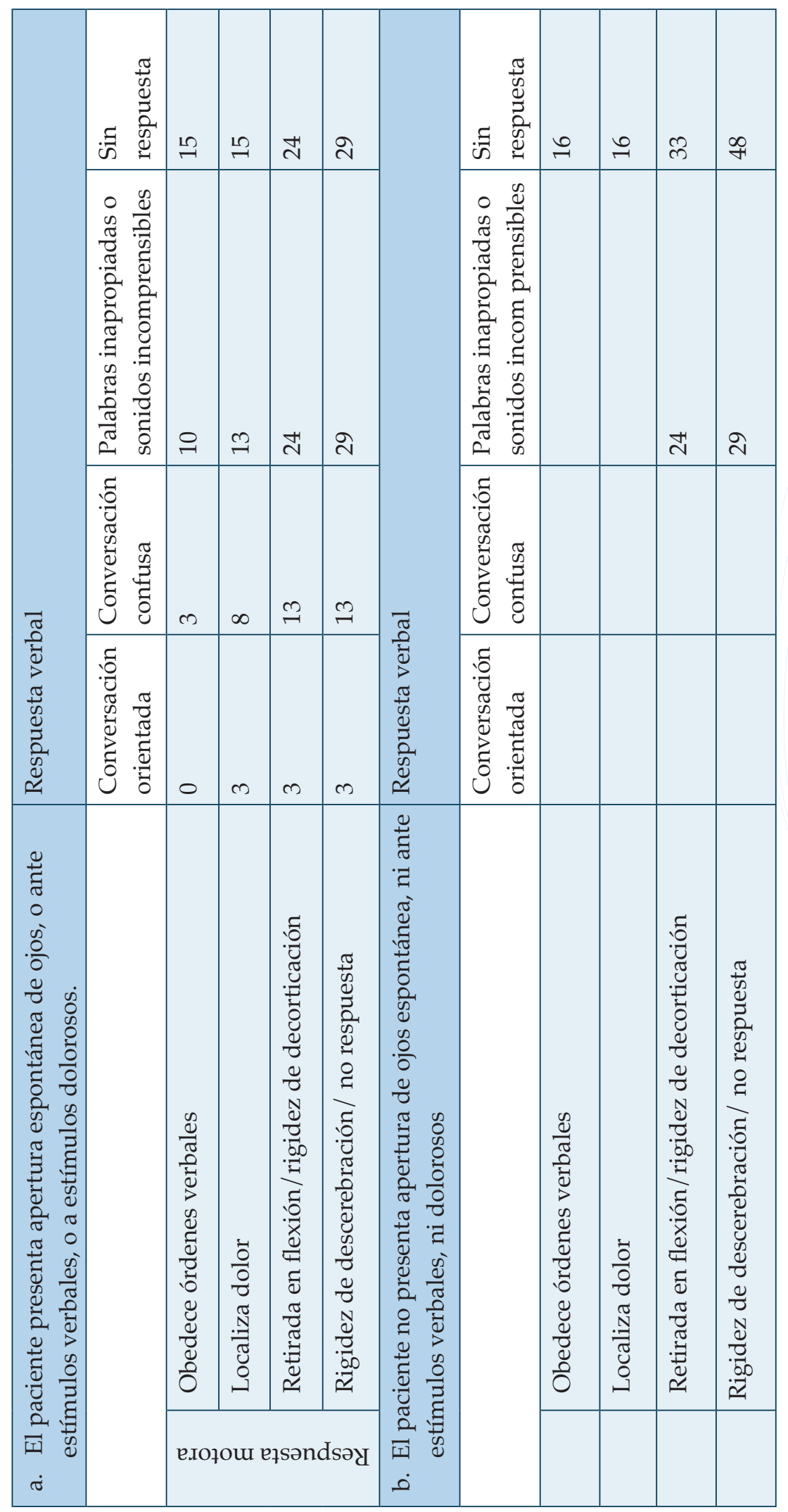




\section{Puntaje fisiológico agudo simplificado II (SAPS II)}

Consta de 12 variables fisiológicas, 3 variables de estado de salud previo, la edad, y una variable indicando la causa de admisión en la UCI (médica o quirúrgica-programada o urgente). Se determina en las primeras 24 horas de ingreso en la UCI, escogiendo el peor valor de cada variable fisiológica durante ese período. Las enfermedades crónicas que puntúan en el estado de salud previo son SIDA, carcinoma metastásico y neoplasia hematológica.

\begin{tabular}{|c|c|c|}
\hline VARIABLE & RANGO & PUNTOS \\
\hline \multirow{6}{*}{ Edad del paciente } & $<40$ años & 0 \\
\hline & 40-59 años & 7 \\
\hline & 60-69 años & 12 \\
\hline & 70-74 años & 15 \\
\hline & 75-79 años & 16 \\
\hline & $\geq 80$ años & 18 \\
\hline \multirow{3}{*}{ Tipo de admisión } & Cirugía Programada & 0 \\
\hline & Médico & 6 \\
\hline & Cirugía no programada & 8 \\
\hline \multirow{2}{*}{ Temperatura } & $<39^{\circ} \mathrm{C},<102.2^{\circ} \mathrm{F}$ & 0 \\
\hline & $\geq 39^{\circ} \mathrm{C}, \geq 102.2^{\circ} \mathrm{F}$ & 3 \\
\hline \multirow{4}{*}{ Presión arterial sistólica } & $\geq 200 \mathrm{mmHg}$ & 2 \\
\hline & $100-199 \mathrm{mmHg}$ & 0 \\
\hline & $70-99 \mathrm{mmHg}$ & 5 \\
\hline & $<70 \mathrm{mmHg}$ & 13 \\
\hline \multirow{5}{*}{ Frecuencia cardíaca } & $\geq 160 \mathrm{lpm}$ & 7 \\
\hline & $120-159 \mathrm{lpm}$ & 4 \\
\hline & 70-119 lpm & 0 \\
\hline & 40-69 lpm & 2 \\
\hline & $<40 \mathrm{lpm}$ & 11 \\
\hline \multirow{5}{*}{ Escala de coma de Glasgow } & $14-15$ & 0 \\
\hline & $11-13$ & 5 \\
\hline & $9-10$ & 7 \\
\hline & $6-8$ & 13 \\
\hline & $<6$ & 26 \\
\hline
\end{tabular}




\begin{tabular}{|c|c|c|}
\hline \multirow{3}{*}{ Producción de orina } & $\geq 1 \mathrm{~L} / 24$ hora & 0 \\
\hline & $0.5-0.999 \mathrm{~L} / 24$ hora & 4 \\
\hline & $<0.5 \mathrm{~L} / 24$ hora & 11 \\
\hline \multirow{3}{*}{ Contaje de leucocitos } & $<1000 / \mathrm{mm}^{3}$ & 12 \\
\hline & $1000-19,000 / \mathrm{mm}^{3}$ & 0 \\
\hline & $\geq 20,000 / \mathrm{mm}^{3}$ & 3 \\
\hline \multirow{3}{*}{ Nitrógeno ureico en sangre } & $\geq 30 \mathrm{mmol} / \mathrm{L}, \geq 84 \mathrm{mg} / \mathrm{dL}$ & 10 \\
\hline & $10-29.9 \mathrm{mmol} / \mathrm{L}, 28-83 \mathrm{mg} / \mathrm{dL}$ & 6 \\
\hline & $<10 \mathrm{mmol} / \mathrm{L},<28 \mathrm{mg} / \mathrm{dL}$ & 0 \\
\hline \multirow{3}{*}{ Nivel de potasio } & $<3 \mathrm{mEq} / \mathrm{L}$ & 3 \\
\hline & 3-4.9 mEq/L & 0 \\
\hline & $\geq 5 \mathrm{mEq} / \mathrm{L}$ & 3 \\
\hline \multirow{3}{*}{ Nivel de sodio } & $<125 \mathrm{mEq} / \mathrm{L}$ & 5 \\
\hline & $125-144 \mathrm{mEq} / \mathrm{L}$ & 0 \\
\hline & $\geq 145 \mathrm{mEq} / \mathrm{L}$ & 1 \\
\hline \multirow{3}{*}{ Nivel de bicarbonato } & $<15 \mathrm{mEq} / \mathrm{L}$ & 6 \\
\hline & $15-19 \mathrm{mEq} / \mathrm{L}$ & 3 \\
\hline & $\geq 20 \mathrm{mEq} / \mathrm{L}$ & 0 \\
\hline \multirow{3}{*}{ Nivel de bilirrubina } & $<4 \mathrm{mg} / \mathrm{dL},<68.4 \mathrm{micromol} / \mathrm{L}$ & 0 \\
\hline & $\begin{array}{l}4-5.9 \mathrm{mg} / \mathrm{dL}, 68.4-102.5 \\
\text { micromol/L }\end{array}$ & 4 \\
\hline & $\geq 6 \mathrm{mg} / \mathrm{dL}, \geq 102.6 \mathrm{micromol} / \mathrm{L}$ & 9 \\
\hline \multirow{3}{*}{$\begin{array}{l}\mathrm{PaO} 2 / \mathrm{FiO} 2 \text { (Si está } \\
\text { ventilado mecánicamente o } \\
\text { recibiendo CPAP.) }\end{array}$} & $<100 \mathrm{mmHg}$ & 11 \\
\hline & $100-199 \mathrm{mmHg}$ & 9 \\
\hline & $\geq 200 \mathrm{mmHg}$ & 6 \\
\hline \multirow{2}{*}{$\begin{array}{l}\text { Síndrome de Inmuno- } \\
\text { Deficiencia Adquirida }\end{array}$} & Sí & 17 \\
\hline & No & 0 \\
\hline \multirow{2}{*}{ Carcinoma metastásico } & Sí & 9 \\
\hline & No & 0 \\
\hline \multirow{2}{*}{ Malignidad hematológica } & Sí & 10 \\
\hline & $\mathrm{No}$ & 0 \\
\hline
\end{tabular}




\section{Puntaje fisiológico agudo simplificado III (SAPS III)}

Es una actualización de SAPS II, capaz de establecer un pronóstico de mortalidad hospitalaria en base a los datos disponibles al ingreso en UCI.

\begin{tabular}{|c|c|c|}
\hline VARIABLE & RANGO & PUNTOS \\
\hline \multirow{6}{*}{${ }^{*}$ Edad del paciente } & $<40$ años & 0 \\
\hline & 40-59 años & 5 \\
\hline & 60-69 años & 9 \\
\hline & 70-74 años & 13 \\
\hline & 75-79 años & 15 \\
\hline & $\geq 80$ años & 18 \\
\hline \multirow{2}{*}{ Temperatura } & $<35^{\circ} \mathrm{C},<102.2^{\circ} \mathrm{F}$ & 7 \\
\hline & $\geq 35^{\circ} \mathrm{C}, \geq 102.2^{\circ} \mathrm{F}$ & 0 \\
\hline \multirow{4}{*}{ Presión arterial sistólica } & $\geq 120 \mathrm{mmHg}$ & 0 \\
\hline & $70-119 \mathrm{mmHg}$ & 3 \\
\hline & $40-69 \mathrm{mmHg}$ & 8 \\
\hline & $<40 \mathrm{mmHg}$ & 11 \\
\hline \multirow{4}{*}{ Frecuencia cardíaca } & $\geq 160 \mathrm{lpm}$ & 7 \\
\hline & $120-159$ lpm & 5 \\
\hline & $<120 \mathrm{lpm}$ & 0 \\
\hline & $<120$ lpm & 5 \\
\hline \multirow{3}{*}{$\begin{array}{l}\text { Escala de coma de } \\
\text { Glasgow }\end{array}$} & $<2$ & 0 \\
\hline & $2-5$ & 4 \\
\hline & $\geq 6$ & 5 \\
\hline \multirow{2}{*}{ Contaje de leucocitos } & $<15$ & 0 \\
\hline & $\geq 15$ & 2 \\
\hline \multirow{4}{*}{ Contaje de plaquetas } & $<20$ & 13 \\
\hline & $20-49$ & 8 \\
\hline & $50-99$ & 5 \\
\hline & $>100$ & 0 \\
\hline \multirow{2}{*}{$\begin{array}{l}\text { Concentración del ión } \\
\text { Hidrógeno }\end{array}$} & $\leq 7.25$ & 3 \\
\hline & $>7.25$ & 0 \\
\hline
\end{tabular}




\begin{tabular}{|c|c|c|}
\hline \multirow{3}{*}{ Niveles de bilirrubina } & $<2 \mathrm{mg} / \mathrm{dL},<34.2 \mathrm{micromol} / \mathrm{L}$ & 0 \\
\hline & $2-5 \mathrm{mg} / \mathrm{dL}, 34.2-102.6$ micromol $/ \mathrm{L}$ & 4 \\
\hline & $\geq 6 \mathrm{mg} / \mathrm{dL}, \geq 102.6 \mathrm{micromol} / \mathrm{L}$ & 5 \\
\hline \multirow{2}{*}{$\begin{array}{l}\mathrm{PaO} / \mathrm{FiO} 2 \text { (en ventilación } \\
\text { mecánica.) }\end{array}$} & $<100 \mathrm{mmHg}$ & 11 \\
\hline & $\geq 100$ & 7 \\
\hline \multirow{2}{*}{$\begin{array}{l}\mathrm{PaO} 2 \text { (no ventilación } \\
\text { mecánica) }\end{array}$} & $<100 \mathrm{mmHg}$ & 11 \\
\hline & $\geq 100$ & 7 \\
\hline \multirow{2}{*}{ Carcinoma metastásico } & Sí & 9 \\
\hline & No & 0 \\
\hline \multirow{3}{*}{$\begin{array}{l}\text { Duración del tiempo de } \\
\text { estanacia en UCI }\end{array}$} & $<14$ días & 0 \\
\hline & 14-27 días & 6 \\
\hline & >28 días & 7 \\
\hline \multirow{4}{*}{ Comorbilidades } & Terapia contra el cáncer & 3 \\
\hline & $\begin{array}{l}\text { Insuficiencia cardíaca crónica, } \\
\text { neoplasias hematológicas. }\end{array}$ & 6 \\
\hline & $\begin{array}{l}\text { Cirrosis, síndrome de } \\
\text { inmudeficiencia adquirida }\end{array}$ & 8 \\
\hline & Cáncer & 11 \\
\hline \multirow{3}{*}{$\begin{array}{l}\text { Localización } \\
\text { intrahospitalaria antes del } \\
\text { ingreso en la UCI }\end{array}$} & Sala de emergencia & 5 \\
\hline & Otra UCI & 7 \\
\hline & Otra & 8 \\
\hline $\begin{array}{l}\text { Uso de otras opciones } \\
\text { terapéuticas importantes } \\
\text { antes del ingreso en la } \\
\text { UCI }\end{array}$ & Drogas vasoactivas & 3 \\
\hline \multirow{2}{*}{ Admisión en UCI } & Planeada & 0 \\
\hline & No planificada & 3 \\
\hline
\end{tabular}




\begin{tabular}{|c|c|c|}
\hline \multirow{10}{*}{ Razones de la admisión } & $\begin{array}{l}\text { Cardiovascular: alteraciones del } \\
\text { ritmo }\end{array}$ & -5 \\
\hline & Neurológico: convulsiones & -4 \\
\hline & $\begin{array}{l}\text { Cardiovascular: shock } \\
\text { hemorrágico hipovolémico, shock } \\
\text { no hemorrágico hipovolémico. } \\
\text { Digestivo: abdomen agudo, otro. }\end{array}$ & 3 \\
\hline & $\begin{array}{l}\text { Neurológico: coma, estupor, } \\
\text { paciente obtundido, alteraciones } \\
\text { de la vigilancia, confusión, } \\
\text { agitación, delirio. }\end{array}$ & 4 \\
\hline & $\begin{array}{l}\text { Cardiovascular: shock séptico. / } \\
\text { Cardiovascular: shock anafiláctico, } \\
\text { shock mixto e indefinido. }\end{array}$ & 5 \\
\hline & Hepática: insuficiencia hepática & 6 \\
\hline & $\begin{array}{l}\text { Neurológico: déficit neurológico } \\
\text { focal. }\end{array}$ & 7 \\
\hline & Digestivo: pancreatitis severa & 9 \\
\hline & $\begin{array}{l}\text { Neurológico: efecto de la masa } \\
\text { intracraneal. }\end{array}$ & 10 \\
\hline & Todos los otros & 0 \\
\hline \multirow{3}{*}{ Estatus quirúrgico } & Programado & 0 \\
\hline & Sin cirujía & 5 \\
\hline & Emergencia & 6 \\
\hline \multirow{5}{*}{$\begin{array}{l}\text { Sitio anatómico de la } \\
\text { cirugía }\end{array}$} & $\begin{array}{l}\text { Cirugía de transplante: hígado, } \\
\text { riñón, páncreas, riñón y páncreas, } \\
\text { otra trasplantación. }\end{array}$ & -11 \\
\hline & $\begin{array}{l}\text { Trauma: otra, aislado (incluye } \\
\text { tórax, abdomen, extremidad). } \\
\text { Trauma: múltiple }\end{array}$ & -8 \\
\hline & $\begin{array}{l}\text { Cirugía cardíaca: } \text { CABG } \text { sin } \\
\text { reparación valvular. }\end{array}$ & -6 \\
\hline & $\begin{array}{l}\text { Neurocirugía: } \\
\text { cerebrovascular. }\end{array}$ & 5 \\
\hline & Todos los otros & 0 \\
\hline
\end{tabular}




\begin{tabular}{|l|l|c|}
\hline \multirow{2}{*}{ Infección aguda } & Nosocomial & 4 \\
\cline { 2 - 3 } & Respiratoria & 5 \\
\hline
\end{tabular}

lpm: latidos por minuto; $\mathrm{PaO}$ 2: presión parcial de oxígeno arterial; FiO 2: fracción de oxígeno inspirado.

${ }^{*}$ Cada paciente obtiene 16 puntos por una admisión con los puntos indicados sumados o restados para cada diagnóstico.

MORTALIDAD SEGÚN SAPS III (interpretación de resultados)

\begin{tabular}{|c|c|}
\hline PUNTUACIÓN & MORTALIDAD (\%) \\
\hline Hasta 21 & $1-10$ \\
\hline $\mathbf{2 2 - 4 4}$ & $10-20$ \\
\hline $\mathbf{4 4 - 5 2}$ & $20-30$ \\
\hline $\mathbf{5 3 - 5 7}$ & $30-40$ \\
\hline $\mathbf{5 8 - 6 2}$ & $40-50$ \\
\hline $\mathbf{6 3 - 6 7}$ & $50-60$ \\
\hline $\mathbf{6 8 - 7 2}$ & $60-70$ \\
\hline $\mathbf{7 3 - 7 8}$ & $70-80$ \\
\hline $\mathbf{7 9 - 8 6}$ & $80-90$ \\
\hline $\mathbf{8 7 - 9 6}$ & $90-95$ \\
\hline $\mathbf{9 1 - 1 1 2}$ & $95-99$ \\
\hline $\mathbf{1 1 3 - 1 5 9}$ & 100 \\
\hline $\mathbf{1 6 0 - 2 2 9}$ & \\
\hline
\end{tabular}

\section{MODELO DE PREDICCIÓN DE MORTALIDAD II (MPM II)}

Describe la severidad de la enfermedad en pacientes admitidos a las UCI, evalúa la efectividad de diferentes UCI estandarizadas de acuerdo con la severidad de la enfermedad de los pacientes admitidos, compara diferentes estrategias de tratamiento haciendo ajustes acordes con la severidad de la enfermedad y establece un marco de referencia objetivo para la toma de decisiones sobre la admisión de pacientes a las UCI o para fines pronósticos una vez admitidos. 


\begin{tabular}{|c|c|c|}
\hline VARIABLES & RANGOS & PUNTOS \\
\hline \multicolumn{3}{|l|}{ Edad del paciente ${ }^{*}$} \\
\hline \multirow{2}{*}{ ¿Admisión quirúrgica médica o no programada? } & Sí & 1 \\
\hline & No & 0 \\
\hline \multirow{2}{*}{ ¿Resucitación cardiopulmonar previa al ingreso? } & Sí & 1 \\
\hline & No & 0 \\
\hline \multirow{2}{*}{$\begin{array}{l}\text { ¿Coma (Glasgow coma escala 3-5)? } \\
\text { (No incluye pacientes cuyo coma se deba a } \\
\text { una sobredosis o que hayan recibido agentes } \\
\text { bloqueadores neuromusculares) }\end{array}$} & Sí & 1 \\
\hline & No & 0 \\
\hline \multirow{2}{*}{ ¿Frecuencia cardíaca $\geq 150$ lpm? } & Sí & 1 \\
\hline & No & 0 \\
\hline \multirow{2}{*}{ ¿Presión arterial sistólica $\leq \mathbf{9 0} \mathrm{mmHg} ?$} & Sí & 1 \\
\hline & No & 0 \\
\hline \multirow{2}{*}{ ¿Ventilación mecánica? } & Sí & 1 \\
\hline & No & 0 \\
\hline \multirow{2}{*}{$\begin{array}{l}\text { ¿Fallo renal agudo? } \\
\text { (No incluye azotemia pre-renal) }\end{array}$} & Sí & 1 \\
\hline & No & 0 \\
\hline \multirow{2}{*}{ ¿Arritmias cardíacas? } & Sí & 1 \\
\hline & No & 0 \\
\hline \multirow{2}{*}{ ¿Accidente cerebrovascular? } & Sí & 1 \\
\hline & No & 0 \\
\hline \multirow{2}{*}{ ¿Efecto de la masa intracraneal? } & Sí & 1 \\
\hline & No & 0 \\
\hline \multirow{2}{*}{ ¿Hemorragia gastrointestinal? } & Sí & 1 \\
\hline & No & 0 \\
\hline \multirow{2}{*}{$\begin{array}{l}\text { ¿Carcinoma metastásico? } \\
\text { (Solo metástasis a distancia; no incluye la } \\
\text { participación de los ganglios linfáticos locales) }\end{array}$} & Sí & 1 \\
\hline & No & 0 \\
\hline
\end{tabular}




\begin{tabular}{|l|c|c|}
\hline \multirow{2}{*}{ ¿Cirrosis? } & Sí & 1 \\
\cline { 2 - 3 } & No & 0 \\
\hline \multirow{2}{*}{ Insuficiencia renal crónica? } & Sí & 1 \\
\cline { 2 - 3 } (Creatinina $>\mathbf{2} \mathbf{~ m g ~ / ~ d L ~ c r o ́ n i c a m e n t e ) ~}$ & No & 0 \\
\hline
\end{tabular}

La edad del paciente no recibe puntos al calcular la puntuación de gravedad; sin embargo, se utiliza en la fórmula para calcular la mortalidad prevista.

\section{MODELO DE PREDICCIÓN DE MORTALIDAD III (MPMO - III)}

\begin{tabular}{|c|c|c|}
\hline VARIABLES & RANGOS & PUNTOS \\
\hline \multicolumn{3}{|l|}{ Edad del paciente* } \\
\hline \multirow{2}{*}{ ¿Admisión quirúrgica médica o no programada? } & Sí & 1 \\
\hline & No & 0 \\
\hline \multirow{2}{*}{ ¿Resucitación cardiopulmonar previa al ingreso? } & Sí & 1 \\
\hline & No & 0 \\
\hline \multirow{2}{*}{$\begin{array}{l}\text { ¿Coma (Glasgow coma escala 3-5)? } \\
\text { (No incluye pacientes cuyo coma se deba a } \\
\text { una sobredosis o que hayan recibido agentes } \\
\text { bloqueadores neuromusculares) }\end{array}$} & Sí & 1 \\
\hline & No & 0 \\
\hline \multirow{2}{*}{ ¿Frecuencia cardíaca $\geq 150$ lpm? } & Sí & 1 \\
\hline & No & 0 \\
\hline \multirow{2}{*}{ ¿Presión arterial sistólica $\leq 90 \mathrm{mmHg} ?$} & Sí & 1 \\
\hline & No & 0 \\
\hline \multirow{2}{*}{ ¿Ventilación mecánica? } & Sí & 1 \\
\hline & No & 0 \\
\hline \multirow{2}{*}{$\begin{array}{l}\text { ¿Fallo renal agudo? } \\
\text { (No incluye azotemia pre-renal) }\end{array}$} & Sí & 1 \\
\hline & No & 0 \\
\hline \multirow{2}{*}{ ¿Arritmias cardíacas? } & Sí & 1 \\
\hline & No & 0 \\
\hline \multirow{2}{*}{ ¿Accidente cerebrovascular? } & Sí & 1 \\
\hline & No & 0 \\
\hline \multirow{2}{*}{ ¿Efecto de la masa intracraneal? } & Sí & 1 \\
\hline & No & 0 \\
\hline
\end{tabular}




\begin{tabular}{|c|c|c|}
\hline \multirow{2}{*}{ ¿Hemorragia gastrointestinal? } & Sí & 1 \\
\hline & No & 0 \\
\hline \multirow{2}{*}{$\begin{array}{l}\text { ¿Carcinoma metastásico? } \\
\text { (Solo metástasis a distancia; no incluye la } \\
\text { participación de los ganglios linfáticos locales) }\end{array}$} & Sí & 1 \\
\hline & No & 0 \\
\hline \multirow{2}{*}{ ¿Cirrosis? } & Sí & 1 \\
\hline & No & 0 \\
\hline \multirow{2}{*}{$\begin{array}{l}\text { ¿Insuficiencia renal crónica? } \\
\text { (Creatinina> } 2 \mathrm{mg} / \mathrm{dL} \text { crónicamente) }\end{array}$} & Sí & 1 \\
\hline & No & 0 \\
\hline \multirow{2}{*}{$\begin{array}{l}\text { ¿En un centro de cuidados agudos o crónicos antes } \\
\text { del ingreso en la UCI? }\end{array}$} & Sí & 1 \\
\hline & No & 0 \\
\hline \multirow{2}{*}{$\begin{array}{l}\text { ¿Tiempo entre hospitalización e ingreso en la } \\
\text { UCI }>1 \text { día? }\end{array}$} & Sí & 1 \\
\hline & No & 0 \\
\hline \multirow{2}{*}{ ¿Estado completo de reanimación? } & Sí & 1 \\
\hline & No & 0 \\
\hline
\end{tabular}

${ }^{*}$ La edad del paciente no recibe puntos al calcular la puntuación de gravedad; sin embargo, se utiliza en la fórmula para calcular la mortalidad prevista.

\section{DISFUNCIÓN ORGÁNICA}

Evaluación de la insuficiencia orgánica relacionada con la sepsis (SOFA)

Evalúa de manera secuencial la gravedad de la disfunción orgánica en pacientes que estaban críticamente enfermos de la sepsis. Evalúa las siguientes características clínicas.

- Sistema respiratorio: la relación entre la tensión arterial de oxígeno y la fracción de oxígeno inspirado ( $\mathrm{PaO} 2$ / FiO2).

- Sistema cardiovascular: la cantidad de medicamento vasoactivo necesaria para prevenir la hipotensión.

- Sistema hepático: el nivel de bilirrubina.

- Sistema de coagulación: la concentración de plaquetas.

- Sistema neurológico: la puntuación de coma de Glasgow.

- Sistema renal: la creatinina sérica o la producción de orina. 
Sepsis: un aumento en la puntuación SOFA $\geq 2$ se asocia con una mortalidad de $\geq 10 \%$.

Shock séptico: pacientes con una puntuación SOFA $\geq 2$ que también tienen un requisito de vasopresor y un lactato elevado $>2 \mathrm{mmol} / \mathrm{L}$ (> $18 \mathrm{mg} / \mathrm{dL}$ ) a pesar de una reanimación con líquidos adecuada tiene un pronóstico de mortalidad del $40 \%$.

\begin{tabular}{|c|c|c|c|c|c|}
\hline Puntuación SOFA & 0 & 1 & 2 & 3 & 4 \\
\hline $\begin{array}{c}\text { Respiración: } \\
\mathrm{PaO} 2 / \mathrm{FIO} 2 \text { (mm } \\
\mathrm{Hg} \text { ) o } \\
\mathrm{SaO} 2 / \mathrm{FIO} 2\end{array}$ & $>400$ & $\begin{array}{l}\text { Menor o } \\
\text { igual a } \\
400\end{array}$ & $\begin{array}{c}\text { Menor o } \\
\text { igual a } 300\end{array}$ & $\begin{array}{l}\text { Menor o igual a } \\
200 \text { con soporte } \\
\text { respiratorio }\end{array}$ & $\begin{array}{c}\text { Menor o igual a } \\
100 \text { con soporte } \\
\text { respiratorio }\end{array}$ \\
\hline $\begin{array}{l}\text { Coagulación: } \\
\text { Plaquetas } x 1.000\end{array}$ & Mayor a 150 & $\begin{array}{l}\text { Menor o } \\
\text { igual a } \\
150\end{array}$ & $\begin{array}{l}\text { Menor o } \\
\text { igual a } 100\end{array}$ & $\begin{array}{c}\text { Menor o igual } \\
\text { a } 50\end{array}$ & $\begin{array}{l}\text { Menor o igual } \\
\text { a } 20\end{array}$ \\
\hline $\begin{array}{l}\text { Hepático: } \\
\text { Bilirrubina } m g / \\
\text { dl(umol/L) }\end{array}$ & $\begin{array}{c}\text { Menor que } \\
1,2(<20)\end{array}$ & $\begin{array}{l}1,2-1.9 \\
(20-32)\end{array}$ & $\begin{array}{r}2,0-5,9 \\
(33-101)\end{array}$ & $\begin{array}{l}6,0-11,9 \\
(102-204)\end{array}$ & $\begin{array}{l}\text { Mayor o igual } \\
12 \\
\text { (>o igual 205) }\end{array}$ \\
\hline Cardiovascular & $\begin{array}{c}\text { No hiperten- } \\
\text { sión }\end{array}$ & $\begin{array}{c}\mathrm{PAM}<70 \\
\mathrm{mmHg}\end{array}$ & $\begin{array}{l}\text { Dopamina a } \\
<\text { o igual a } 5 \\
\text { o Dobutami- } \\
\text { na a cualqui- } \\
\text { er dosis }\end{array}$ & $\begin{array}{c}\text { Dopamina } \\
\text { a dosis }> \\
5 \text { o Epinefrina a } \\
\leq 0,1 \text { o Norepine- } \\
\text { frina } a \leq 0,1\end{array}$ & $\begin{array}{c}\text { Dopamina } \\
\text { a dosis de } \\
>15 \text { o Epine- } \\
\text { frina }>0,1 \text { o } \\
\text { Norepinefrina a } \\
>0,1\end{array}$ \\
\hline $\begin{array}{c}\text { Neurológico: } \\
\text { Glasgow }\end{array}$ & 15 & $13-14$ & 10-12 & $6-9$ & $<6$ \\
\hline $\begin{array}{l}\text { Renal: creatinina } \\
m g / d l(u m o l / L) o \\
\text { diuresis/24h }\end{array}$ & $<1,2(<110)$ & $\begin{array}{c}1,2-1,9 \\
(110-170)\end{array}$ & $\begin{array}{r}2,0-3,4 \\
(171-299)\end{array}$ & $\begin{array}{c}3,5-4,9 \\
(300-440) \text { o }<500 \\
\mathrm{ml} / \text { día }\end{array}$ & $\begin{array}{l}>0 \text { igual 5,0 } \\
\text { (>440) ó } \\
<200 \mathrm{ml} / \text { día }\end{array}$ \\
\hline $\begin{array}{c}\text { SOFA total (6 } \\
\text { items) }\end{array}$ & & & & & \\
\hline
\end{tabular}

PaO2: presión arterial de oxígeno; FIO2: fracción de oxígeno inspirado; $\mathrm{SaO}$, Saturación arterial de oxígeno periférico; PAM, presión arterial media; a). PaO2/FIO2 es relación utilizada preferentemente, pero si no está disponible usaremos la SaO2/FIO2; b). Medicamentos vasoactivos administrados durante al menos 1 hora (dopamina $y$ norepinefrina como ug/ $\mathrm{kg} / \mathrm{min}$ ) para mantener la PAM por encima de $65 \mathrm{mmHg}$. 


\section{INTERPRETACIÓN ESACALA DE SOFA}

\begin{tabular}{|c|c|}
\hline MÁXIMA PUNTUACIÓN SOFA & MORTALIDAD $\%$ \\
\hline $\mathbf{0}$ a $\mathbf{6}$ & $<10 \%$ \\
\hline $\mathbf{7}$ a $\mathbf{9}$ & $15-20 \%$ \\
\hline $\mathbf{1 0}$ a $\mathbf{1 2}$ & $40-50 \%$ \\
\hline $\mathbf{1 3}$ a $\mathbf{1 4}$ & $50-60 \%$ \\
\hline $\mathbf{1 5}$ & $>80 \%$ \\
\hline $\mathbf{1 5}$ a $\mathbf{2 4}$ & $>90 \%$ \\
\hline
\end{tabular}

\section{INFLAMACIÓN/SEPSIS}

Q-SOFA: es una herramienta para ayudar a identificar pacientes con sepsis temprana fuera de la UCI.

\section{EVALUACIÓN SECUENCIAL DE FALLA ORGÁNICA, Rápida (Quick SOFA o qSOFA)}

\begin{tabular}{|c|c|}
\hline ¿Qué evalúa? & ¿Cómo Evalúa? \\
\hline $\begin{array}{l}\text { A los pacientes se les asigna } \\
\text { un punto para cada una de } \\
\text { las siguientes características } \\
\text { clínicas; } \\
\text { frecuencia respiratoria } \geq 22 \text { / } \\
\text { min } \\
\text { alteración del nivel de } \\
\text { conciencia } \leq 13 \text { puntos } \\
\text { presión arterial sistólica } \leq 100 \\
\text { mmHg. }\end{array}$ & $\begin{array}{l}\text { Cuando al menos } 2 / 3 \text { criterios están } \\
\text { presentes, muestra una validez predictiva } \\
\text { similar al SOFA para la detección de } \\
\text { pacientes con sospecha de sepsis y } \\
\text { probabilidad de presentar una evolución } \\
\text { desfavorable. }\end{array}$ \\
\hline
\end{tabular}

-Una puntuación baja no elimina la posibilidad de sepsis, por lo que se recomienda seguir evaluando al paciente, si sigue siendo sospechoso. Una puntuación alta induce a adoptar medidas más concretas de tratamiento, con medición de lactato, evaluación con SOFA, tratamiento antibiótico y fluidoterapia. 


\section{EVALUACIÓN DE FUENTES COMUNES DE SEPSIS}

\begin{tabular}{|c|c|}
\hline & Puntos \\
\hline \multicolumn{2}{|l|}{ Temperatura } \\
\hline $350>40^{\circ} \mathrm{C}$ & 2 \\
\hline $35.1-36^{\circ} \mathrm{C}$ o $39-39.9^{\circ} \mathrm{C}$ & 1 \\
\hline $36.1-38.9^{\circ} \mathrm{C}$ & 0 \\
\hline \multicolumn{2}{|l|}{ Hipotensión } \\
\hline $\begin{array}{l}\text { Hipotensión aguda con descenso de presión arterial (PA) sistólica } \\
\text { y diastólica > } 30 \text { y } 20 \mathrm{mmHg} \text {, respectivamente, o uso de agentes } \\
\text { vasopresores, o PA sistólica }<90 \mathrm{mmHg} \text {. }\end{array}$ & 2 \\
\hline Ventalización mecánica & 2 \\
\hline Fracaso cardíaco & 4 \\
\hline \multicolumn{2}{|l|}{ Estado mental } \\
\hline Alerta & 0 \\
\hline Desorientación & 1 \\
\hline Estupor & 3 \\
\hline Coma & 4 \\
\hline
\end{tabular}

\begin{tabular}{|c|c|c|}
\hline $\begin{array}{l}\text { Sistema de } \\
\text { Evaluación }\end{array}$ & VENTAJAS & DESVENTAJAS \\
\hline Apache IV & $\begin{array}{l}\text { Coeficientes actualizados } \\
\text { regularmente. } \\
\text { Proporciona algoritmos para la } \\
\text { predicción de LOS. } \\
\text { Algoritmo específico para predecir } \\
\text { mortalidad en pacientes con cirugía } \\
\text { CABG. } \\
\text { Menos propensos a verse afectados } \\
\text { por la mezcla de casos. }\end{array}$ & $\begin{array}{l}\text { Muestra de desarrollo } \\
\text { restringida a un país. } \\
\text { Recopilación de datos } \\
\text { más compleja. } \\
\text { Gran carga de } \\
\text { abstracción. } \\
\text { Sistema de puntuación } \\
\text { propietario.* }\end{array}$ \\
\hline
\end{tabular}




\begin{tabular}{|c|c|c|}
\hline MPM $_{0}$-III & $\begin{array}{l}\text { Baja carga de abstracción. } \\
\text { Menos propensos a la variabilidad } \\
\text { interobservador. } \\
\text { Al usar menos datos fisiológicos, } \\
\text { puede ser preferido cuando } \\
\text { los recursos de laboratorio son } \\
\text { constreñido. }\end{array}$ & $\begin{array}{l}\text { Muestra de desarrollo } \\
\text { ma y orita riame n te } \\
\text { restringida a un país. } \\
\text { Más susceptible a los } \\
\text { efectos de mezcla de } \\
\text { casos. }\end{array}$ \\
\hline SAPS 3 & $\begin{array}{l}\text { La menor carga de abstracción. } \\
\text { Menos propensos a la variabilidad } \\
\text { interobservador. } \\
\text { Muestra de desarrollo de } 35 \text { países } \\
\text { de los cinco continentes. } \\
\text { Ecuaciones personalizadas para } \\
\text { predecir la mortalidad hospitalaria } \\
\text { según siete regiones geográficas } \\
\text { diferentes. } \\
\text { Uso potencial paraelbenchmarking } \\
\text { internacional. }\end{array}$ & $\begin{array}{l}\text { No proporciona } \\
\text { estimación para LOS. } \\
\text { Algunas ecuaciones } \\
\text { regionales se } \\
\text { desarrollaron utilizando } \\
\text { un tamaño de muestra } \\
\text { relativamente bajo. }\end{array}$ \\
\hline
\end{tabular}

Apache: fisiología aguda y evaluación crónica de la salud; LOS: duración de la estancia; CABG: injerto de bypass de arteria coronaria; MPM: modelo de probabilidad de mortalidad; SAPS: puntaje de fisiología aguda simplificada.

* Corporación CERNER ha hecho públicos los algoritmos de puntuación. 


\section{SEDOANALGESIA}

ESCALA DE SEDACIÓN DE RAMSAY: es la escala más utilizada en la práctica clínica para valorar el grado de sedación de los pacientes, sin embargo, no ha sido validada. Muy sencilla, puede ser utilizada por personal no entrenado.

\begin{tabular}{|l|l|}
\hline \multicolumn{2}{|l|}{ ESCALA DE RAMSAY } \\
\hline Nivel 1 & Paciente ansioso agitado o inquieto \\
\hline Nivel 2 & Paciente cooperador, orientado, tranquilo \\
\hline Nivel 3 & Paciente dormido, responde a órdenes \\
\hline Nivel 4 & Paciente dormido, respuesta rápida a estímulos \\
\hline Nivel 5 & Paciente dormido, respuesta lenta a estímulos \\
\hline Nivel 6 & Paciente dormido, ausencia de respuesta \\
\hline
\end{tabular}

\section{ESCALA DE AGITACIÓN/SEDACIÓN DE RICHMOND}

Es una escala validada y fiable al realizar mediciones seriadas, en estudios bien diseñados y amplios. Separa el estímulo verbal y físico. De esa forma el grado de sedación del paciente se puede categorizar de acuerdo al grado de estímulo.

\begin{tabular}{|l|l|l|}
\hline PUNTOS & TÉRMINO & DESCRIPCIÓN \\
\hline $\mathbf{4}$ & Agresivo & $\begin{array}{l}\text { Abiertamente combativo, violento; peligro } \\
\text { inmediato para el personal. }\end{array}$ \\
\hline $\mathbf{3}$ & Muy agitado & $\begin{array}{l}\text { Se quita o tira del tubo o los catéteres; } \\
\text { agresivo. }\end{array}$ \\
\hline $\mathbf{2}$ & Agitado & $\begin{array}{l}\text { Frecuentes movimientos sin propósito. } \\
\text { Lucha con el respirador }\end{array}$ \\
\hline $\mathbf{1}$ & Intranquilo & $\begin{array}{l}\text { Ansioso pero los movimientos no son } \\
\text { agresivos o vigorosos. }\end{array}$ \\
\hline $\mathbf{0}$ & Alerta y tranquilo \\
\hline
\end{tabular}




\begin{tabular}{|c|c|c|c|}
\hline-1 & Somnoliento & $\begin{array}{l}\text { No completamente } \\
\text { alerta, pero tiene un } \\
\text { despertar mantenido } \\
\text { (apertura de los ojos } \\
\text { y contacto visual) a la } \\
\text { llamada (<10seg). }\end{array}$ & \multirow{3}{*}{$\begin{array}{l}\text { ESTÍMULO } \\
\text { VERBAL }\end{array}$} \\
\hline-2 & Sedación ligera & $\begin{array}{l}\text { Se despierta } \\
\text { brevemente, contacta } \\
\text { con los ojos a la } \\
\text { llamada (<10seg). }\end{array}$ & \\
\hline-3 & $\begin{array}{l}\text { Sedación } \\
\text { moderada }\end{array}$ & $\begin{array}{l}\text { Movimiento o apertura } \\
\text { de los ojos a la llamada } \\
\text { (pero no contacto } \\
\text { visual) }\end{array}$ & \\
\hline-4 & $\begin{array}{l}\text { Sedación } \\
\text { profunda }\end{array}$ & $\begin{array}{l}\text { No responde a la } \\
\text { llamada, pero se } \\
\text { mueve o abre los ojos a } \\
\text { la estimulación física. }\end{array}$ & \multirow{2}{*}{$\begin{array}{l}\text { ESTÍMULO } \\
\text { FÍSICO }\end{array}$} \\
\hline-5 & No despertable & $\begin{array}{l}\text { No responde a la } \\
\text { llamada ni a estímulos } \\
\text { físicos. }\end{array}$ & \\
\hline
\end{tabular}




\section{POLITRAUMATISMO Y QUEMADOS}

INJURY SEVERITY SCORE: es una escala que permite evaluar la severidad de las lesiones, considerada una herramienta útil para predecir pronóstico en injurias graves.

\begin{tabular}{|c|c|c|c|}
\hline Región & $\begin{array}{c}\text { Descripción de la } \\
\text { lesión }\end{array}$ & $\begin{array}{c}\text { Escala } \\
\text { abreviada } \\
\text { de lesiones } \\
\text { (AIS) }\end{array}$ & $\begin{array}{c}\text { Tres primeros } \\
\text { cuadrados }\end{array}$ \\
\hline Cabeza y cuello & $\begin{array}{l}\text { Contusión } \\
\text { cerebral }\end{array}$ & 3 & 9 \\
\hline Cara & No hay lesión & 0 & \\
\hline Pecho & $\begin{array}{l}\text { Pecho } \\
\text { traumatizado }\end{array}$ & 4 & 16 \\
\hline & $\begin{array}{l}\text { Menor contusión } \\
\text { del hígado }\end{array}$ & 2 & \\
\hline Abdomen & $\begin{array}{l}\text { Ruptura compleja } \\
\text { del bazo }\end{array}$ & 5 & 25 \\
\hline Extremidades & Fractura de fémur & 3 & \\
\hline Externas & No existe lesión & 0 & \\
\hline \multicolumn{3}{|c|}{ Escala de lesión severa } & 50 \\
\hline \multicolumn{3}{|l|}{ ISS SCORE } & \\
\hline $1-8$ & \multicolumn{2}{|l|}{ Menor } & \\
\hline $9-15$ & \multicolumn{2}{|l|}{ Moderada } & \\
\hline $16-24$ & \multicolumn{2}{|l|}{ Seria } & \\
\hline $25-49$ & \multicolumn{2}{|l|}{ Severa } & \\
\hline $50-74$ & \multicolumn{2}{|l|}{ Crítica } & \\
\hline 75 & \multicolumn{2}{|l|}{ Crítica Máxima } & \\
\hline
\end{tabular}

\begin{tabular}{|c|c|}
\hline ESACALA AIS & LESIÓN \\
\hline 1 & Menor \\
\hline 2 & Moderada \\
\hline 3 & Seria \\
\hline 4 & Severa \\
\hline 5 & Crítica \\
\hline 6 & Supervivible \\
\hline
\end{tabular}




\section{PENETRATING TRAUMA INDEX (PATI)}

\begin{tabular}{|c|c|c|c|}
\hline Órgano & \multicolumn{2}{|c|}{ Factor de riesgo } & Gravedad de la lesión \\
\hline \multirow[t]{3}{*}{ Duodeno } & 5 & $\begin{array}{l}1 . \\
2 . \\
3 .\end{array}$ & $\begin{array}{l}\text { Mínima lesión de la pared } \\
\text { Desgarro de }<25 \% \text { de la pared } \\
\text { Desgarro de }>25 \% \text { de la circunferencia }\end{array}$ \\
\hline & & 4. & $\begin{array}{l}\text { Desgarro de la pared duodenal y } \\
\text { desvacularización }\end{array}$ \\
\hline & & 5. & Duodeno-pancreatectomía \\
\hline \multirow[t]{4}{*}{ Páncreas } & 5 & $\begin{array}{l}1 . \\
2 .\end{array}$ & $\begin{array}{l}\text { Lesión tangencial } \\
\text { Desgarro sin sección del conducto }\end{array}$ \\
\hline & & 3. & Sección con lesión distal del conducto \\
\hline & & 4. & Lesión proximal del conducto \\
\hline & & 5. & Pancreato - duodenectomía \\
\hline \multirow[t]{4}{*}{ Hígado } & 4 & $\begin{array}{l}1 . \\
2 .\end{array}$ & $\begin{array}{l}\text { Lesión periférica no sangrante } \\
\text { Lesión central, o hemorrágica, o desgarro } \\
\text { menor }\end{array}$ \\
\hline & & 3. & $\begin{array}{l}\text { Lesión parenquimatosa mayor o ligadura de } \\
\text { art. hepática }\end{array}$ \\
\hline & & 4. & Lobectomía \\
\hline & & 5. & $\begin{array}{l}\text { Lobectomía con reparación de la cava o } \\
\text { lesión bilobar }\end{array}$ \\
\hline \multirow[t]{3}{*}{ Colon } & 4 & $\begin{array}{l}1 . \\
2 .\end{array}$ & $\begin{array}{l}\text { Lesión serosa } \\
\text { Lesión parietal simple }\end{array}$ \\
\hline & & $\begin{array}{l}3 . \\
4 .\end{array}$ & $\begin{array}{l}\text { Desgarro de }<25 \% \text { de la circunferencia } \\
\text { Desgarro de }>25 \% \text { de la circunferencia }\end{array}$ \\
\hline & & 5. & Sección completa o desvascularización \\
\hline $\begin{array}{l}\text { Grandes } \\
\text { vasos }\end{array}$ & 4 & $\begin{array}{l}1 . \\
2 . \\
3 .\end{array}$ & $\begin{array}{l}\text { Desgarro de }<25 \% \text { de la pared } \\
\text { Desgarro de }>25 \% \text { de la pared } \\
\text { Sección completa }\end{array}$ \\
\hline
\end{tabular}




\begin{tabular}{|c|c|c|c|}
\hline & & 4. & Interposición de injerto o Bypass \\
\hline & & 5. & Ligadura \\
\hline \multirow[t]{4}{*}{ Bazo } & 3 & $\begin{array}{l}1 . \\
2 .\end{array}$ & $\begin{array}{l}\text { Lesión no sangrante } \\
\text { Utilización de coagulación o agentes } \\
\text { hemostáticos }\end{array}$ \\
\hline & & 3. & Lesión mínima o sutura \\
\hline & & 4. & Resección parcial \\
\hline & & 5. & Esplenectomía \\
\hline \multirow[t]{4}{*}{ Riñón } & 3 & $\begin{array}{l}1 . \\
2 .\end{array}$ & $\begin{array}{l}\text { Lesión no sangrante Lesión mínima o } \\
\text { suturada }\end{array}$ \\
\hline & & 3. & Lesión profunda del parénquima \\
\hline & & 4. & Lesión de la pelvis o calicial \\
\hline & & 5. & Nefrectomía \\
\hline \multirow[t]{3}{*}{$\begin{array}{l}\text { Vías } \\
\text { biliares }\end{array}$} & 3 & $\begin{array}{l}1 . \\
2 .\end{array}$ & Contusión Colecistectomía \\
\hline & & $\begin{array}{l}3 . \\
4 .\end{array}$ & $\begin{array}{l}\text { Desgarro de }>25 \% \text { de vía biliar principal } \\
\text { Desgarro de }>25 \text { de la vía biliar principal }\end{array}$ \\
\hline & & 5. & Reconstrucción bilio-entérica \\
\hline $\begin{array}{l}\text { Intestino } \\
\text { delgado }\end{array}$ & 2 & $\begin{array}{l}1 . \\
2 . \\
3 . \\
4 . \\
5 .\end{array}$ & $\begin{array}{l}\text { Lesión parietal simple } \\
\text { Lesión perforante } \\
\text { Desgarro de }>25 \% \text { de la pared o 2-3 lesiones } \\
\text { Desgarro de }>25 \% \text { de la pared o 4-5 lesiones } \\
\begin{array}{l}\text { Sección transversal con pérdida tisular o } \\
\text { desvascularización }\end{array}\end{array}$ \\
\hline
\end{tabular}




\begin{tabular}{|c|c|c|c|}
\hline Estómago & 2 & $\begin{array}{l}1 . \\
2 . \\
3 . \\
4 . \\
5 .\end{array}$ & $\begin{array}{l}\text { Lesión parietal simple } \\
\text { Perforación } \\
\text { Desgarro menor } \\
\text { Resección parcial } \\
\text { Resección }>35 \%\end{array}$ \\
\hline Uréter & 2 & $\begin{array}{l}1 . \\
2 . \\
3 . \\
4 . \\
5 .\end{array}$ & $\begin{array}{l}\text { Contusión } \\
\text { Laceración } \\
\text { Desgarro menor } \\
\text { Resección segmentaria } \\
\text { Reconstrucción }\end{array}$ \\
\hline Vejiga & 1 & $\begin{array}{l}1 . \\
2 . \\
3 . \\
4 . \\
5 .\end{array}$ & $\begin{array}{l}\text { Lesión parietal simple } \\
\text { Perforación } \\
\text { Desgarro } \\
\text { Resección parcial } \\
\text { Reconstrucción }\end{array}$ \\
\hline Hueso & 1 & $\begin{array}{l}1 . \\
2 . \\
3 . \\
4 . \\
5 .\end{array}$ & $\begin{array}{l}\text { Lesión perióstica } \\
\text { Lesión cortical } \\
\text { Penetrante } \\
\text { Intra-articular } \\
\text { Pérdida ósea importante }\end{array}$ \\
\hline $\begin{array}{c}\text { Pequeños } \\
\text { vasos }\end{array}$ & 1 & $\begin{array}{l}1 . \\
2 . \\
3 . \\
4 . \\
5 .\end{array}$ & $\begin{array}{l}\text { Pequeño hematoma no sangrante } \\
\text { Gran hematoma no sangrante } \\
\text { Sutura } \\
\text { Ligadura de vasos aislados } \\
\text { Ligadura de vasos definidos }\end{array}$ \\
\hline
\end{tabular}




\section{Clasificación de Marshall de la lesión cerebral traumática por TAC}

\begin{tabular}{|l|l|}
\hline $\begin{array}{l}\text { Lesión axonal difusa } \\
\text { tipo I }\end{array}$ & No signos de lesión cerebral \\
\hline $\begin{array}{l}\text { Lesión axonal difusa } \\
\text { tipo II }\end{array}$ & $\begin{array}{l}\text { Presencia de cisternas basales, desviación de la línea } \\
\text { media }<5 \mathrm{~mm} \text { y } / \text { o ausencia de lesiones hiperdensas o } \\
\text { en mosaico }>25 \mathrm{ml} .\end{array}$ \\
\hline $\begin{array}{l}\text { Lesión axonal difusa } \\
\text { tipo III (swelling) }\end{array}$ & $\begin{array}{l}\text { Compresión o desaparición de cisternas de la base, } \\
\text { desviación de la línea media }>5 \mathrm{~mm} \text {, ausencia de } \\
\text { lesión hiperdensa o en mosaico }>25 \mathrm{ml} .\end{array}$ \\
\hline $\begin{array}{l}\text { Lesión axonal difusa } \\
\text { tipo IV (efecto masa } \\
\text { o shift) }\end{array}$ & $\begin{array}{l}\text { Desviación de la línea media }>5 \mathrm{~mm} \text {, no lesiones } \\
\text { hiperdensas o en mosaico }>25 \mathrm{ml} .\end{array}$ \\
\hline Lesión quirúrgica & Todas las lesiones quirúrgicas (hematoma). \\
\hline $\begin{array}{l}\text { Lesión no quirúrgica } \\
\text { (masa no evacuada) }\end{array}$ & $\begin{array}{l}\text { Lesiones hiperdensas o en mosaico }>25 \mathrm{ml} \text { (no } \\
\text { indicación quirúgica). }\end{array}$ \\
\hline
\end{tabular}




\section{NEUROLOGÍA}

\begin{tabular}{|c|c|}
\hline \multicolumn{2}{|c|}{ ESCALA DE COMA DE GLASGOW } \\
\hline Escala & Respue sta a la apertura de los ojos (O) \\
\hline 1 & No existe apertura de los ojos \\
\hline 2 & Abertura de los ojos al dolor \\
\hline 3 & $\begin{array}{l}\text { Abertura de los ojos como respuesta al } \\
\text { llamado }\end{array}$ \\
\hline 4 & Apertura de los ojos de manera espontánea \\
\hline Escala & Respuesta Verbal (V) \\
\hline 1 & No existe respuesta verbal \\
\hline 2 & Sonidos incompresibles \\
\hline 3 & Palabras que no tienen sentido \\
\hline 4 & Confundido \\
\hline 5 & Orientado \\
\hline Escala & Respuesta Motora (M) \\
\hline 1 & No existe respuesta motora \\
\hline 2 & Extensión al dolor \\
\hline 3 & Flexión al dolor \\
\hline 4 & Retracción al dolor \\
\hline 5 & Dolor localizado \\
\hline 6 & Obedece a las ordenes \\
\hline $\begin{array}{l}\text { Un puntaje de coma de } 13 \\
\text { lesión moderada y } 8 \text { o meno }\end{array}$ & $\begin{array}{l}\text { la con lesión cerebral leve, 9-12 es una } \\
\text { cerebro. }\end{array}$ \\
\hline
\end{tabular}

\section{ESCALA DE COMA DE GLASGOW PEDIÁTRICO}

\begin{tabular}{|c|c|c|c|}
\hline Escala & Apertura de los ojos & Respuesta verbal & Respuesta moto ra \\
\hline 6 & - & - & $\begin{array}{l}\text { Normal espontáneo, } \\
\text { obedece } \\
\text { comandos, } \\
\text { localizado }\end{array}$ \\
\hline 5 & - & $\begin{array}{l}\text { Palabras apropiadas } \\
\text { a la edad, sonrisa } \\
\text { social }\end{array}$ & Dolor localizado \\
\hline 4 & Espontánea & $\begin{array}{l}\text { Llora, } \\
\text { consolable }\end{array}$ & $\begin{array}{l}\text { Se retira del estímulo } \\
\text { del dolor }\end{array}$ \\
\hline 3 & A la voz & $\begin{array}{l}\text { Persistentemente } \\
\text { irritable }\end{array}$ & $\begin{array}{l}\text { Flexión de la postura } \\
\text { al dolor }\end{array}$ \\
\hline 2 & Al dolor & Inquieta agitada & $\begin{array}{l}\text { Extensión de la } \\
\text { postura al dolor }\end{array}$ \\
\hline 1 & Ninguna & Ninguna & Ninguna \\
\hline
\end{tabular}

\section{- Escala de Coma de Glasgow}

\begin{tabular}{|l|l|}
\hline \multicolumn{1}{|c|}{ Categoría } & \multicolumn{1}{c|}{ Definición } \\
\hline (1) Muerte & $\begin{array}{l}\text { Sin respuesta y sin habla durante semanas, meses } \\
\text { o hasta la muerte; puede tener ciclo vigilia-sueño } \\
\text { después de 2-3 semanas. }\end{array}$ \\
\hline $\begin{array}{l}\text { (2) Estado vegetativo } \\
\text { persistente }\end{array}$ & $\begin{array}{l}\text { Pacientes dependientes de apoyo diario, debido a } \\
\text { incapacidad mental o física o una combinación de } \\
\text { ambas. }\end{array}$ \\
\hline $\begin{array}{l}\text { (3) Incapacidad grave } \\
\text { (consciente pero } \\
\text { incapacitado) }\end{array}$ & \\
\hline
\end{tabular}


(4) Incapacidad moderada (incapacitado pero independiente)
Capaz de trabajar en un ambiente protegido y viajar en transporte público.

Las limitaciones incluyen diferentes grados de disfasia, hemiparesia o ataxia, así como déficit intelectual y de memoria, y cambios en la personalidad.

Incorporación a la vida normal. Puede haber déficits neurológicos o psicológicos menores.

La escala de coma de Glasgow es una escala clínica y sirve para cuantificar la profundidad y duración del deterioro de la conciencia después de un trauma craneoencefálico. Constituye un buen indicador de la severidad de las lesiones cerebrales y de la evolución de las mismas. La escala de Glasgow puede ser deprimida por alteraciones fisiológicas extracraneales, como el shock hipovolémico. Para su aplicación comprende medidas independientes de tres aspectos funcionales: la respuesta motora, la respuesta verbal y la apertura de los ojos.

Escala de Hunt y Hess de hemorragia subaracnoidea: es una manera de clasificar la severidad de una hemorragia subaracnoidea no traumática, puesto que cuando la condición clínica del paciente se evalúa basado con los criterios de Hunt y Hess para el momento de la cirugía se demuestra una correlación específica con el resultado del tratamiento quirúrgico. La escala se correlaciona con un índice de mortalidad asociada a los diferentes grados, numerados del 1 al 5 .

\begin{tabular}{|c|l|}
\hline Grado & \multicolumn{1}{|c|}{ Criterios } \\
\hline I & Asintomático o mínima cefalea y ligera rigidez de nuca. \\
\hline II & $\begin{array}{l}\text { Cefalea moderada o intensa, rigidez de nuca sin déficit neurológico (a } \\
\text { excepción de parálisis de pares craneales). }\end{array}$ \\
\hline III & Letargia, confusión o ligero déficit focal. \\
\hline IV & $\begin{array}{l}\text { Estupor, hemiparesia moderada o grave, probable rigidez de descerebración } \\
\text { y alteraciones vegetativas. }\end{array}$ \\
\hline V & Coma profundo, rigidez de descerebración. \\
\hline
\end{tabular}

- Grados de Fisher de hemorragia subaracnoidea: es un criterio utilizado en medicina para predecir el vasoespasmo posterior a una hemorragia subaracnoidea utilizando una tomografía axial computarizada. Predice el riesgo de vasoespasmo después de una hemorragia subaracnoidea. 


\begin{tabular}{|c|c|}
\hline Grado & Descripción \\
\hline Grado I & Ausencia de sangre en espacio subaracnoideo \\
\hline Grado II & HSA difusa \\
\hline Grado III & HSA mayor de 1 mm de espesor \\
\hline Grado IV & Hemorragia intracerebral o intraventricular con o sin sangre difusa \\
\hline
\end{tabular}

\section{- Delirio del enfermo crítico, Método de evaluación de la confusión} en paciente de UCI (CAM-UCI): es ampliamente usado a nivel internacional para el diagnóstico de delirium en pacientes críticos que reciben ventilación mecánica (Tobara, 2009).

\section{CAM-UCI}

1. Comienzo agudo o curso fluctuante

¿Hay evidencia de un cambio agudo en su estado mental con respecto a la situación basal? ó

¿Ha cambiado la conducta del enfermo en las últimas 24 horas? (cambio en la puntuación RASS o en la puntuación de Glasgow).

2. Disminución de la atención

¿Presenta el paciente dificultad para dirigir la atención?

¿Presenta el paciente dificultad para mantener y desviar la atención?

\section{Alteraciones cognitivas}

¿Es el pensamiento del paciente desorganizado e incoherente?

¿Contesta a preguntas y obedece órdenes durante la entrevista?

4. Alteración de conciencia

¿Está el paciente alerta e hipervigilante? (RASS > 0)

¿Está el paciente somnoliento o estuporoso? (RASS entre -1 y -3; grados menores de reactividad se consideran coma).

CAM-ICU: $1+2+(3$ o 4$)$ 


\section{RESPIRATORIO}

- TRALI (Transfusion-related acute lung injury): es una escala que permite diagnosticar lesión pulmonar aguda producida por transfusión (Transfusión-Related Acute Lung Injury - TRALI), es un síndrome clínico que se presenta como hipoxemia aguda y edema pulmonar no cardiogénico durante o tras una transfusión de productos hemáticos. Es un proceso relativamente infrecuente pero que puede constituir una amenaza vital.

\begin{tabular}{|l|}
\hline I. Criterios de TRALI \\
LPA \\
Hipoxemienzo agudo \\
$\mathrm{PaO} / \mathrm{FiO} 2<300$ \\
$\mathrm{SaO} 2<90 \%$ respirando aire ambiente \\
Infiltrados bilaterales en la radiografía de tórax \\
Sin evidencia de aumento de presión en aurícula izquierda \\
No LPA antes de la transfusión (sobrecarga de volumen) \\
Durante o en las 6 primeras horas de la transfusión \\
No relación temporal con otro factor de riesgo de LPA \\
\hline
\end{tabular}

\section{Posible TRALI}

LPA

No LPA previa a la transfusión

Durante $o$ en las 6 primeras horas de la transfusión

Clara relación en el tiempo con otro factor de riesgo de LPA

Clasificación del EPOC, según gravedad: en la clasificación del EPOC se evalúa: 
El Estadio 0 se caracteriza por tos crónica y producción de esputo. La función respiratoria medida por espirometría es aún normal.

- El Estadio I caracterizado por limitación leve al flujo aéreo, y usualmente, aunque no siempre, tos crónica y producción de esputo. En este estadio el enfermo puede no ser consciente de su situación clínica.

- El Estadio II producido por un empeoramiento de la limitación al flujo aéreo y usualmente un empeoramiento sintomático con disnea de esfuerzo. Es la fase en la que el enfermo típicamente consulta por disnea o por una exacerbación de su enfermedad. La división en estadios IIA o IIB se basa en el hecho que las exacerbaciones son vistas con mayor frecuencia en pacientes con FEV1 $<50 \%$ del predicho. La presencia de exacerbaciones repetidas tiene un impacto en la calidad de vida y requiere un manejo adecuado.

- El Estadio III presenta una limitación grave al flujo aéreo, la presencia de signos de fallo respiratorio o fracaso ventricular derecho. La calidad de vida en este estadio está limitada y las exacerbaciones pueden comprometer la vida.

\begin{tabular}{|c|c|}
\hline Estadio & Características \\
\hline 0 & $\begin{array}{l}\text { Espirometría normal } \\
\text { Síntomas crónicos: tos, expectoración. }\end{array}$ \\
\hline I: EPOC leve & $\begin{array}{l}\text { FEV1/FVC }<70 \% \\
\text { FEV1 }<80 \% \text { del predicho } \\
\text { Con o sin síntomas crónicos: tos, expectoración. }\end{array}$ \\
\hline $\begin{array}{l}\text { II: } \quad \text { EPOC } \\
\text { moderado }\end{array}$ & $\begin{array}{l}\text { FEV1 } / \mathrm{FVC}<70 \% \\
30 \%>\mathrm{FEV} 1<80 \% \text { del predicho } \\
\text { II A: } 50 \%>\mathrm{FEV} 1<80 \% \text { del predicho } \\
\text { II B: } 30 \%>\mathrm{FEV} 1<50 \% \text { del predicho con o sin síntomas } \\
\text { crónicos: tos, expectoración. }\end{array}$ \\
\hline $\begin{array}{l}\text { III: } \\
\text { grave }\end{array}$ & $\begin{array}{l}\text { FEV1 } / \mathrm{FVC}<70 \% \\
\text { FEV1 }<30 \% \text { del predicho } \\
\text { O presencia de fallo respiratorio }\left({ }^{*}\right) \text {, o signos clínicos de fallo } \\
\text { cardíaco derecho }\end{array}$ \\
\hline
\end{tabular}


- Criterios para el diagnóstico de embolia grasa: son un conjunto de signos y síntomas y se establecen de la siguiente manera, se requieren al menos un criterio mayor y cuatro menores para establecer el diagnóstico.

\begin{tabular}{|l|c|}
\hline \multicolumn{1}{|c|}{ SÍNTOMA/SIGNO } & PUNTUACIÓN \\
\hline Petequias & 5 \\
\hline Infiltrados alveolares difusos & 4 \\
\hline Hipoxemia & 3 \\
\hline Confusión & 1 \\
\hline Fiebre $>38^{\circ} \mathrm{C}$ & 1 \\
\hline Frec. cardíaca $>120 \mathrm{lpm}$ & 1 \\
\hline Frec. Respiratoria $>30 \mathrm{rpm}$ & 1 \\
\hline
\end{tabular}

\section{CARDIOLÓGICOS Y VASCULARES}

- Infarto agudo de miocardio: clasificación de Forrester: describen 4 subgupos, dependiendo de las variables índice cardíaco y presión de enclavamiento pulmonar, además correlacionan datos hemodinámicos y datos clínicos en los pacientes que sufren una insuficiencia cardíaca tras un infarto de miocardio.

\begin{tabular}{|l|l|l|}
\hline \multirow{2}{*}{ Índice cardíaco } & \multicolumn{2}{|l|}{ Presión de enclavamiento pulmonar } \\
\cline { 2 - 3 } & $<18$ & $>\mathbf{1 8}$ \\
\hline$>2,2$ & I & II \\
\hline$<2,2$ & III & IV \\
\hline
\end{tabular}

-Anginainestable:clasificación de Braunwald:Se trata de unaclasificación clínica que puede relacionarse con la enfermedad subyacente, es útil para para pronosticar supervivencia total. Para categorizar la población que presenta angina inestable Braunwald propone esta clasificación que enfoca tres aspectos:

1. La gravedad de las manifestaciones clínicas.

2. Las circunstancias clínicas en las que ocurre la angina inestable.

3. El hecho de que los episodios isquémicos sintomáticos estén o no acompañados de cambios electrocardiográficos transitorios. 


\begin{tabular}{|c|c|c|c|}
\hline GRAVEDAD & A & B & C \\
\hline $\begin{array}{c}\text { Desarrollada } \\
\text { con situación } \\
\text { extracardíaca } \\
\text { que intensifica la } \\
\text { isquemia } \\
\text { miocárdica (angina } \\
\text { secundaria). }\end{array}$ & $\begin{array}{c}\text { Desarrollada } \\
\text { en ausencia } \\
\text { de problemas } \\
\text { extracardíacos } \\
\text { (angina primaria). }\end{array}$ & $\begin{array}{c}\text { Desarrollada } \\
\text { en las dos } \\
\text { semanas post } \\
\text { infarto agudo } \\
\text { de miocardio. }\end{array}$ \\
\hline $\begin{array}{c}\text { I (comienzo o } \\
\text { progresiva). No } \\
\text { reposo. }\end{array}$ & IA & IB & IC \\
\hline $\begin{array}{c}\text { II (reposo } \\
\text { subaguda), } \\
\text { último mes en } \\
\text { reposo, no en } \\
\text { las últimas } 48 \\
\text { horas. }\end{array}$ & IIA & IIB & IIC \\
\hline $\begin{array}{c}\text { III (reposo } \\
\text { aguda), últimas } \\
48 \text { horas en } \\
\text { reposo }\end{array}$ & IIIA & IIIB & IIIC \\
\hline
\end{tabular}

Escala de trombolisis en infarto agudo de miocardio (TIMI) de perfusión coronaria: es una escala que se crea con el objetivo de poder predecir el riesgo de muerte en estos sujetos. El recuento de flujo TIMI es simple, reproducible, cuantificable y permite la estandarización entre los distintos laboratorios.

\begin{tabular}{|c|l|}
\hline GRADO & \multicolumn{1}{|c|}{ CRITERIOS } \\
\hline 0 & $\begin{array}{l}\text { Oclusión completa en la arteria responsable del infarto. No existe } \\
\text { flujo anterógrado. }\end{array}$ \\
\hline 1 & $\begin{array}{l}\text { Penetración de contraste por el lugar de la oclusión, pero sin } \\
\text { perfusión en distal en el lecho coronario. }\end{array}$ \\
\hline 2 & $\begin{array}{l}\text { Perfusión de toda la arteria coronaria, pero el flujo anterógrado } \\
\text { es lento. }\end{array}$ \\
\hline 3 & Flujo anterógrado despejado. \\
\hline
\end{tabular}


Clasificación funcional de la insuficiencia cardíaca de la New York Heart Association: La escala de la NYHA se usa de manera rutinaria en la gradación de la insuficiencia cardíaca. La clasificación es útil en la evaluación de la situación en fase inicial y después del tratamiento, en el seguimiento de los enfermos. Se ha relacionado de manera inversa con la supervivencia. Una limitación es la baja concordancia interobservador para la asignación de clases, por lo que se han propuesto otras clasificaciones funcionales con escalas de actividad que han tenido menor difusión en la práctica clínica.

\begin{tabular}{|l|l|}
\hline CLASE & \multicolumn{1}{|c|}{ DESCRIPCIÓN } \\
\hline I & $\begin{array}{l}\text { Sin limitación. Las actividades físicas habituales no causan disnea, } \\
\text { cansancio o palpitaciones. }\end{array}$ \\
\hline II & $\begin{array}{l}\text { Ligera limitación de la actividad física. El paciente está bien en } \\
\text { reposo. La actividad física habitual le causa disnea, cansancio, } \\
\text { palpitaciones o angina. }\end{array}$ \\
\hline III & $\begin{array}{l}\text { Limitación marcada de la actividad física. Si bien, el enfermo está } \\
\text { en reposo, las actividades menores le causan síntomas. }\end{array}$ \\
\hline IV & $\begin{array}{l}\text { Incapacidad de cualquier actividad física sin síntomas. Los } \\
\text { síntomas están presentes incluso en reposo. Con cualquier } \\
\text { actividad se incrementa la incomodidad. }\end{array}$ \\
\hline
\end{tabular}

Disección aórtica: clasificación de DeBakey: es una escala que permite evaluar el daño de la aorta, por disección aórtica. Previamente ha sido utilizada la clasificación de DeBakey, que anatómicamente es más descriptiva, pero orienta menos a la hora de dirigir el tratamiento.

\begin{tabular}{|c|l|}
\hline TIPO & LOCALIZACIÓN \\
\hline I & $\begin{array}{l}\text { Se inicia en la aorta ascendente y se extiende distalmente hacia el } \\
\text { arco y la aorta descendente }\end{array}$ \\
\hline II & Comienza y se limita a la aorta ascendente \\
\hline III & $\begin{array}{l}\text { Comienza y se limita a la aorta descendente: } \\
\text { III a: solamente afecta a la aorta torácica } \\
\text { III b: se extiende hasta aorta abdominal }\end{array}$ \\
\hline
\end{tabular}




\section{GASTROENTEROLOGÍA}

- Clasificación de gravedad de Child-Turcotte de la cirrosis hepática: con el desarrollo del trasplante hepático se hizo importante utilizar criterios que permitieran determinar el pronóstico en pacientes individuales con la mayor exactitud posible. Esta escala ayuda a seleccionar pacientes cirróticos para cirugía de shunt portocava. Sus criterios permitían la separación de los pacientes en tres categorías (A, B y C) estratificados de mejor $(\mathrm{A})$ a peor $(\mathrm{C})$ pronóstico.

\begin{tabular}{|l|c|c|c|}
\hline \multirow{2}{*}{ ÍNDICE } & & CLASE & \\
\cline { 2 - 4 } & A & B & C \\
\hline Bilirrubina (mg/dl) & $<2,3$ & $2,3-2,9$ & $>2,9$ \\
\hline Albúmina (g/dl) & $>3,5$ & $3-3,5$ & $<3$ \\
\hline Ascitis & No & $\begin{array}{c}\text { Bien } \\
\text { controlada }\end{array}$ & $\begin{array}{c}\text { Mal } \\
\text { controlada } \\
\text { Avanzada }\end{array}$ \\
\hline Encefalopatía & No & Moderada & Malo \\
\hline Estado nutricional & Excelente & Bueno & . \\
\hline
\end{tabular}

- Clasificación de Foster de hemorragia gastrointestinal: describe hallazgos endoscópicos en pacientes con sangrado digestivo relacionado con patología ulcerosa.

\begin{tabular}{|l|l|l|c|}
\hline \multicolumn{2}{|c|}{ GRADO } & HALLAZGOS ENDOSCÓPICOS & $\begin{array}{c}\text { PROBABILIDAD DE } \\
\text { RESANGRADO }\end{array}$ \\
\hline \multirow{2}{*}{ I } & Ia & Sangrado a arterial & $90 \%$ \\
\cline { 2 - 4 } & Ib & Sangrado en sábana & $45 \%$ \\
\hline \multirow{4}{*}{ II } & IIa & Vaso visible & $20 \%$ \\
\cline { 2 - 4 } & IIb & Coágulo adherido & $10 \%$ \\
\cline { 2 - 4 } & IIc & Mancha negruzca & \\
\hline \multirow{2}{*}{ III } & & Sin sangrado & \\
\hline
\end{tabular}

Índice de gravedad de la tomografía computarizada (Tomografía computarizada dinámica secuencial. Balthazar): el CTSI es un sistema de 10 puntos basado en la valoración del grado inflamación pancreática y peri-pancreática ( $0-2$ puntos), presencia y número de colecciones peri-pancreáticas (0-2 puntos) y la presencia y grado de necrosis del parénquima pancreático (0-6 puntos). 


\begin{tabular}{|l|c|}
\hline COLECCIONES & PUNTUACIÓN \\
\hline Páncreas normal (A) & 0 \\
\hline Aumento de tamaño de la glándula (B) & 1 \\
\hline Inflamación peripáncreática (C) & 2 \\
\hline Colección única (D) & 3 \\
\hline Múltiples colecciones (E) & 4 \\
\hline
\end{tabular}

\begin{tabular}{|l|c|}
\hline NECROSIS & PUNTUACIÓN \\
\hline$<30 \%$ & 2 \\
\hline $30-50 \%$ & 4 \\
\hline$>50 \%$ & 6 \\
\hline Total & 10 \\
\hline
\end{tabular}

- Valoración morfológica de la pancreatitis aguda. Clasificación de Balthazar

\begin{tabular}{|l|l|}
\hline Grado A & Páncreas normal \\
\hline Grado B & $\begin{array}{l}\text { Aumento focal o difuso del tamaño del páncreas incluyendo } \\
\text { contornos irregulares de la glándula, dilatación del conducto } \\
\text { pancreático y colecciones líquidas pequeñas sin evidencia de } \\
\text { enfermedad peri-pancreática. }\end{array}$ \\
\hline Grado C & $\begin{array}{l}\text { Alteraciones pancreáticas intrínsecas asociadas con cambios } \\
\text { inflamatorios en la grasa peri-cardíaca . }\end{array}$ \\
\hline Grado D & Colección líquida o flemón único bien definido \\
\hline Grado E & $\begin{array}{l}\text { Dos o más colecciones líquidas mal definidas o presencia de gas } \\
\text { en el páncreas o adyacente a él. }\end{array}$ \\
\hline
\end{tabular}




\section{CIRUGÍA}

- Criterios para la identificación de pacientes con alto riesgo de presentar una alta incidencia de morbimortalidad postoperatoria (LORENZO, 2006), (Amy Grace Rapsang, 2014), (Mark A Kelley, 2019), (Keegan MT, 2011), (Minne L, 2008), (Maccariello E, 2010), (Salciccioli JD, 2012), (ffrenchO'Carroll R, 2015), (P., 2000), (Marshall LF, 2013).

Enfermedad cardiorespiratoria grave actual o previa (infarto de miocardio, EPOC, ACVA, ICC).

Catástrofe abdominal aguda con inestabilidad hemodinámica: pancreatitis, gangrena o perforación intestinal, sangrado gastrointestinal, perforación víscera hueca.

Fracaso renal agudo: urea $>15 \mathrm{mmol} / \mathrm{L}$, creatinina $>265 \mathrm{mmol} / \mathrm{L}$.

Politrauma grave ( $>$ de 3 órganos o $>$ de 2 sistemas o apertura de dos cavidades corporales).

Edad > de 70 años con evidencia de reserva fisiológica limitada para uno o más órganos vitales.

Shock: $\mathrm{TAM}<60 \mathrm{mmHg}$, $\mathrm{PVC}<15 \mathrm{~cm} \mathrm{H} 2 \mathrm{O}$ y débito urinario $<0,5 \mathrm{ml} / \mathrm{kg} / \mathrm{h}$.

Fracaso respiratorio: $\mathrm{PaO}_{2}<60 \mathrm{~mm} \mathrm{Hg}$ con $\mathrm{FiO}_{2}>0,4$, shunt $>30 \%$, ventilación mecánica $>48 \mathrm{~h}$.

Septicemia, hemocultivo positivo o foco séptico, con inestabilidad hemodinámica asociada.

${ }^{*}$ Cuando se cumplen los criterios expuestos la mortalidad estimada es del 35 al $40 \%$.

\section{METABÓLICO/NUTRICIONAL}

- Estrés metabólico: esta escala es una gradación del estrés según parámetros metabólicos.

\begin{tabular}{|l|l|l|l|}
\hline GRADO & $\mathbf{1}$ & $\mathbf{2}$ & $\mathbf{3}$ \\
\hline Nitrógeno orina $(\mathrm{g} /$ día $)$ & $5-10$ & $10-15$ & $>15$ \\
\hline Glucemia $(\mathrm{mg} / \mathrm{dl})$ & $\begin{array}{l}125 \quad \pm \\
25\end{array}$ & $\begin{array}{l}150 \\
50\end{array}$ & $200 \pm 50$ \\
\hline \multirow{2}{*}{$\mathrm{IVO} 2\left(\mathrm{ml} / \mathrm{mn} / \mathrm{m}^{2}\right)$} & $\begin{array}{l}130 \pm \\
10\end{array}$ & $\begin{array}{l}140 \\
10\end{array}$ & $160 \pm 10$ \\
\hline Resistencia a insulina & No & No/Sí & Sí \\
\hline CR & 0,85 & 0,85 & $0,85-1$ \\
\hline
\end{tabular}

IVO2 = indice de consumo de oxigeno (VO2/superficie corporal). CR = cociente respiratorio. 
Criterio diagnóstico de diabetes insípida/Síndrome de secreción inadecuada de hormona antidiurética (SIADH):

\begin{tabular}{|c|c|c|}
\hline \multicolumn{2}{|l|}{ DIABETES INSÍPIDA } & SIADH \\
\hline \multirow{2}{*}{\multicolumn{2}{|c|}{$\begin{array}{l}\text { Hipernatremia (sodio plasmático > } 145 \\
\mathrm{mEq} / \mathrm{L} \text { ). Hipokaliemia. Hipercalcemia. } \\
\text { Hiperosmolaridad plasmática (> } 295 \mathrm{mOsm} / \\
\text { kg). }\end{array}$}} & $\begin{array}{l}\text { Hiponatremia } \quad \text { (sodio } \\
\text { plasmático }<135 \mathrm{mEq} / \mathrm{L} \text { ) }\end{array}$ \\
\hline & & $\begin{array}{l}\text { Hiposmolaridad plasmática } \\
(<280 \mathrm{mOsm} / \mathrm{kg}) \text {. }\end{array}$ \\
\hline \multicolumn{2}{|c|}{$\begin{array}{l}\text { Hiperuricemia }>5 \mathrm{mg} / \mathrm{dl} \text {, en presencia de } \\
\text { poliuria/polidipsia. }\end{array}$} & Sodio urinario > 30 mEq/L. \\
\hline \multicolumn{2}{|c|}{ Osmolaridad urinaria $<300 \mathrm{mOsm} / \mathrm{kg}$. } & $\begin{array}{l}\text { Osmolaridad urinaria }>100 \\
\mathrm{mOsm} / \mathrm{kg} \text { en presencia de } \\
\text { hiponatremia. }\end{array}$ \\
\hline \multicolumn{2}{|c|}{$\begin{array}{l}\text { Después de administración de } 5 \text { u S.C. de } \\
\text { ADH acuosa o 1-2 } \mu \text { g de dDAVP S.C.: }\end{array}$} & $\begin{array}{l}\text { Ausencia de hipovolemia, } \\
\text { hipotensión, fallo cardíaco, } \\
\text { nefrosis, cirrosis } \\
\text { insuficiencia adrenal, o }\end{array}$ \\
\hline D.I. NEUROGÉNICA & D.I. NEFROGÉNICA & \\
\hline \multirow{3}{*}{$\begin{array}{l}\text { La osmolaridad de } \\
\text { la orina aumenta en } \\
\text { más de un } 50 \% \text {. }\end{array}$} & $\begin{array}{l}\text { La osmolaridad de la } \\
\text { orina aumenta menos } \\
\text { de un } 9 \% .\end{array}$ & $\begin{array}{l}\text { Urea plasmática, ácido úrico, } \\
\text { creatinina y actividad de } \\
\text { renina normal o suprimido. }\end{array}$ \\
\hline & \multirow{2}{*}{$\begin{array}{l}\text { Persiste poliuria } \\
\text { hipotónica. }\end{array}$} & $\begin{array}{l}\text { Cortisol y tiroxina plasmática } \\
\text { normales. }\end{array}$ \\
\hline & & $\begin{array}{l}\text { Sodio urinario }>20 \mathrm{mEq} / \text { día } \\
\text { (no siempre). }\end{array}$ \\
\hline
\end{tabular}


RELACIÓN MÉDICO - PACIENTE CRÍTICAMENTE ENFERMOS GUÍAS DE COMUNICACIÓN CON EL PACIENTE

\section{GUÍAS DE COMUNICACIÓN CON EL PACIENTE}

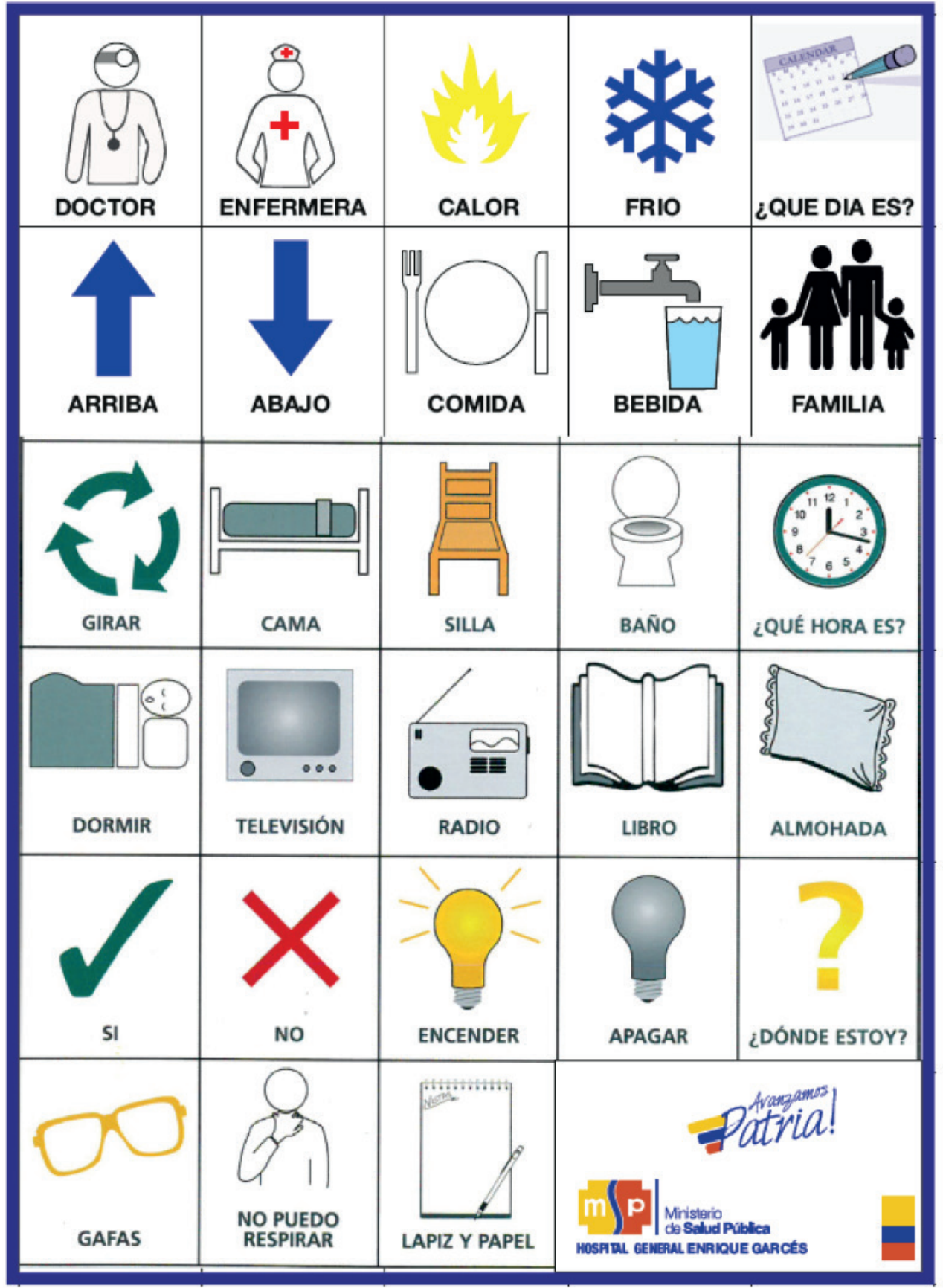




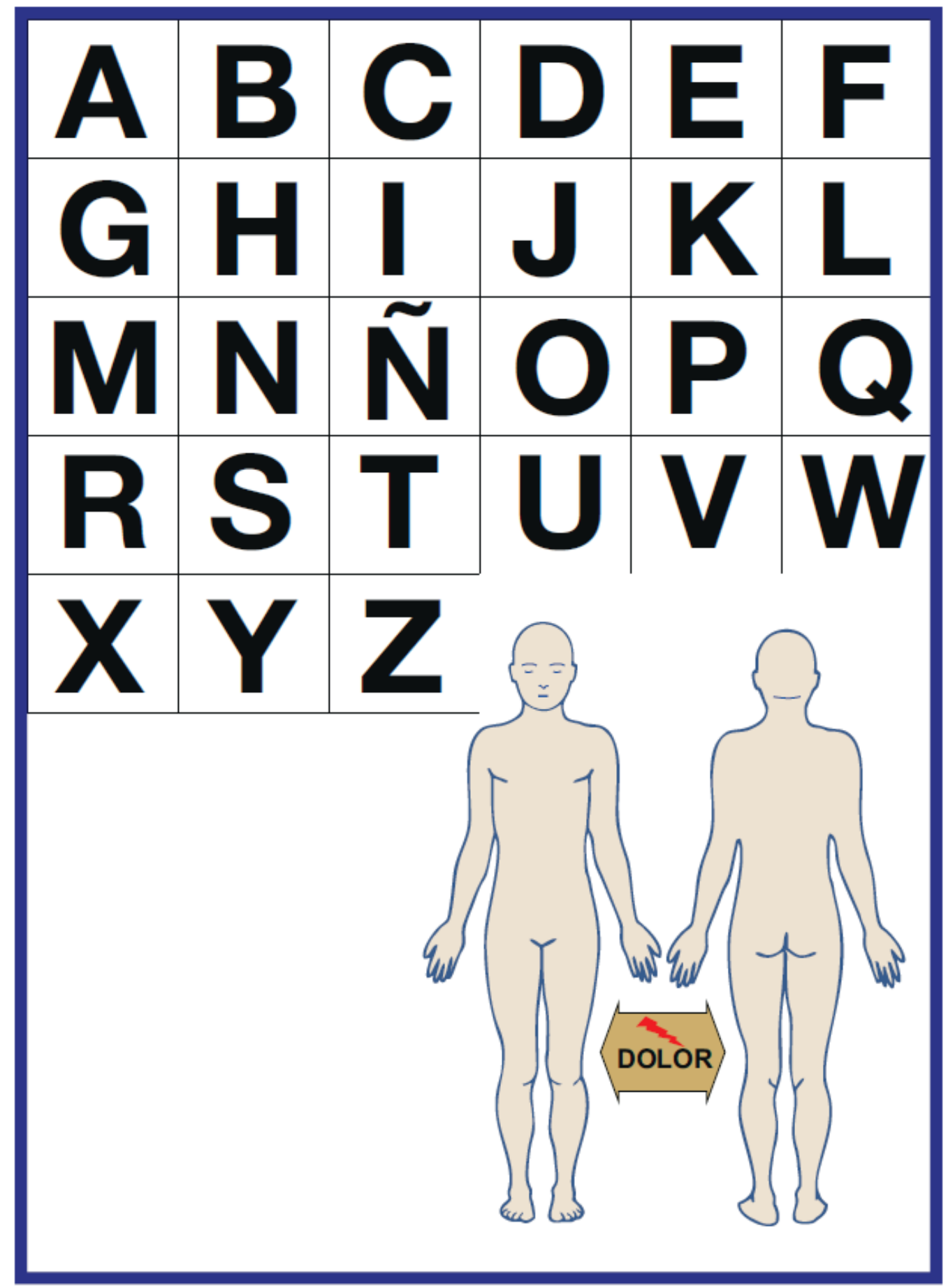




\section{Bibliografía}

Tobara, E. (2009). Metodo para la evaluaci 'on de la confusi'on en la unidad de cuidados intensivos para el diagn'ostico de del'ırium: adaptaci'on cultural y validaci'on de la versi'on en idioma español. Medicna intensiva , 04 (3).

LORENZO, A. G. (2006). Scores pronósticos y Criterios diagnósticosen el paciente crítico (2da edición ed.). Madrid: Ergon.

Amy Grace Rapsang, D. C. (2014). Scoring systems in the intensive care unit: A compendium. Indian Journal of Critical Care Medicine , 18.

Mark A Kelley, M. M. (junio de 2019). Predictive scoring systems in the intensive care unit. Wolters Kluwer.

Keegan MT, G. O. (2011). Severity of illness scoring systems in the intensive care unit. Critic Unit Care, 39 (163).

Minne L, A.-H. A. (2008). Evaluation of SOFA-based models for predicting mortality in the UCI. A systematic review. Crit Care , 12 (161).

Maccariello E, V. C. (2010). SAPS 3 scores at the start of renal replacement therapy predict mortality in critically ill patients with acute kidney injury. Kidney Int , 77 (51).

Salciccioli JD, C. C. (2012). Performance of SAPS II and SAPS III scores in postcardiac cardiac arrest. Minerva Anestesiol , 78 (1341).

ffrench-O'Carroll R, F. S. (2015). Predictors of outcome in decompensated liver disease: validation of the SOFA-L score. Ir Med J , 108 (114).

P., A. L. (2000). Diabetes insípida. En: Manual de Cuidados Intensivos. madrid: Harcourt Brace.

Marshall LF, G. T. (2013). A new classification of head injury based on computerized. J Neurosurg , 75, 514-20.

M. Urina, H. C. (2000). Validación de un modelo para predicción de mortalidad en la Unidad de Cuidado Intensivo . Acta Médica Colombiana , 14 (6).

Dr. Rómulo Soler Vaillant. (2010). EVALUACIÓN PRONOSTICA EN EL TRAUMATIZADO.

Vallejo-Villalobos, D. M. (2012). Embolia. Rev. Mexicana de anestesiología , 35, 15154.

Martos-Benítez, D. I.-P. (2016). Escala TIMI como predictor de muerte en pacientes con infarto miocárdico agudo sin intervención coronaria percutánea. revista de enfermedades cardiovasculares , 8 (2), 111-116.

Hüseyin Incea, C. A. (mayo de 2007). Tratamiento de los síndromes aórticos agudos. rev. española de cardiología , 526-541. 


\section{APÉNDICE III.}

ESCALA DE NEWS 2 MODIFICADA (PUNTUACIÓN NACIONAL DE ALERTA TEMPRANA)SERVICIO DE MEDICINA INTERNA HOSPITAL GENERAL IESS RIOBAMBA

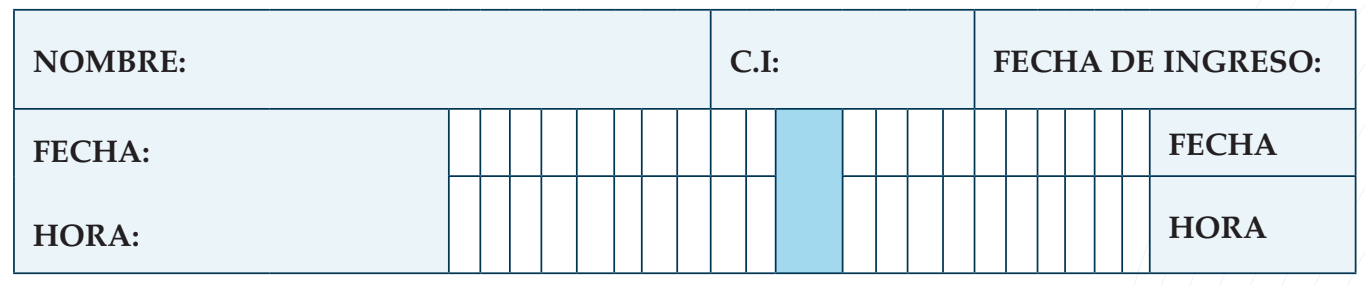

\begin{tabular}{|c|l|l|l|l|l|l|l|l|l|l|l|l|}
\hline \multirow{5}{*}{$\begin{array}{c}\text { Frecuencia } \\
\text { Respiratoria }\end{array}$} & $21-24$ & & & & & 2 & & & & & $21-24$ \\
\hline & $12-20$ & & & & & 0 & & & & & $12-20$ \\
\hline & $9-11$ & & & & & 1 & & & & $9-11$ \\
\hline & $\leq 8$ & & & & 3 & & & & $\leq 8$ \\
\hline
\end{tabular}

\begin{tabular}{|c|c|c|c|}
\hline \multirow{2}{*}{$\begin{array}{l}\text { Necesidad } \\
\text { de oxígeno* }\end{array}$} & $\mathrm{Si}$ & 2 & Sí \\
\hline & $\mathrm{No}$ & 0 & No \\
\hline
\end{tabular}

\begin{tabular}{|c|c|c|c|}
\hline \multirow{6}{*}{ Temperatura } & $\geq 39^{\circ}$ & 2 & $\geq 39^{\circ}$ \\
\hline & $38.1-39^{\circ}$ & 1 & $38.1-39^{\circ}$ \\
\hline & $37.1-38^{\circ}$ & \multirow{2}{*}{0} & $37.1-38^{\circ}$ \\
\hline & $36.1-37^{\circ}$ & & $36.1-37^{\circ}$ \\
\hline & $35.1-36^{\circ}$ & 1 & $35.1-36^{\circ}$ \\
\hline & $\leq 35^{\circ}$ & 3 & $\leq 35^{\circ}$ \\
\hline
\end{tabular}




\begin{tabular}{|c|c|c|c|}
\hline \multirow{14}{*}{$\begin{array}{l}\text { Presión } \\
\text { arterial } \\
\text { sistólica }\end{array}$} & $\geq 220$ & 3 & $\geq 220$ \\
\hline & $201-219$ & \multirow{3}{*}{2} & 201-219 \\
\hline & $181-200$ & & $181-200$ \\
\hline & $161-180$ & & $161-180$ \\
\hline & $141-160$ & 1 & $141-160$ \\
\hline & $121-140$ & 0 & $121-140$ \\
\hline & $111-120$ & & $111-120$ \\
\hline & $101-110$ & 1 & $101-110$ \\
\hline & $91-100$ & 2 & $91-100$ \\
\hline & $81-90$ & \multirow{5}{*}{3} & $81-90$ \\
\hline & $71-80$ & & $71-80$ \\
\hline & $61-70$ & & $61-70$ \\
\hline & $51-60$ & & $51-60$ \\
\hline & $\leq 50$ & & $\leq 50$ \\
\hline \multirow{12}{*}{$\begin{array}{c}\text { Frecuencia } \\
\text { cardiáca }\end{array}$} & $\geq 131$ & 3 & $\geq 131$ \\
\hline & $121-130$ & \multirow{2}{*}{2} & $121-130$ \\
\hline & $111-120$ & & $111-120$ \\
\hline & $101-110$ & 1 & $101-110$ \\
\hline & $91-100$ & & $91-100$ \\
\hline & $81-90$ & 0 & $81-90$ \\
\hline & $71-80$ & & $71-80$ \\
\hline & $61-70$ & & $61-70$ \\
\hline & $51-60$ & & $51-60$ \\
\hline & $41-50$ & 1 & $41-50$ \\
\hline & $31-40$ & \multirow{2}{*}{3} & $31-40$ \\
\hline & $\leq 30$ & & $\leq 30$ \\
\hline
\end{tabular}

\begin{tabular}{|c|c|c|c|}
\hline \multirow{2}{*}{$\begin{array}{c}\text { Nivel } \\
\text { conciencia }\end{array}$} & Alerta & 0 & Alerta \\
\hline & $\mathrm{O} / \mathrm{V} / \mathrm{M}$ & 3 & $\mathrm{O} / \mathrm{V} / \mathrm{M}$ \\
\hline
\end{tabular}




\begin{tabular}{|c|c|c|c|c|c|c|c|c|c|c|c|c|c|c|c|}
\hline $\begin{array}{l}\text { TOTAL } \\
\text { SIRS }\end{array}$ & & & & & & & & & & & & & $\begin{array}{c}\text { TOTAL } \\
\text { SIRS }\end{array}$ \\
\hline $\begin{array}{l}\text { TOTAL } \\
\text { q-SOFA }\end{array}$ & & & & & & & & & & & & & & & $\begin{array}{c}\text { TOTAL } \\
\text { q-SOFA }\end{array}$ \\
\hline
\end{tabular}

\begin{tabular}{|c|c|c|c|c|c|c|c|c|c|c|c|c|c|c|}
\hline $\begin{array}{c}\text { Gasto } \\
\text { Urinario }\end{array}$ & $\begin{array}{c}\mathrm{cc} / \\
\mathrm{kg} / \mathrm{h}\end{array}$ & & & & & & & & & & & & & $\begin{array}{c}\text { Gasto } \\
\text { Urinario }\end{array}$ \\
\hline
\end{tabular}

\begin{tabular}{|c|c|c|c|c|c|c|c|c|c|c|c|c|c|c|}
\hline $\begin{array}{c}\text { Niveles de } \\
\text { Glucemia }\end{array}$ & $\mathrm{mg} / \mathrm{dl}$ & & & & & & & & & & & & & $\begin{array}{c}\text { Niveles } \\
\text { de } \\
\text { Glucemia }\end{array}$ \\
\hline
\end{tabular}

* Paciente $\leq$ de 65 años, sin comorbilidades (insuficiencia cardiaca, fibrosis pulmonar, EPOC, hipertensión pulmonar) los valores normales de saturación de oxígeno serán $\geq 92 \%$. Si el paciente tiene $>$ de 65 años y presenta comorbilidades entonces sus niveles de saturación normal serán entre $88-92 \%$. Valores inferiores a los descritos, serán criterio de necesidad de oxígeno.

** En caso de obtener una puntuación mayor a 4, considere según el caso, también calcular SCORE SIRS y q-SOFA.

\begin{tabular}{|c|c|c|}
\hline $\begin{array}{c}\text { Puntaje news } 2 \\
\text { modificado }\end{array}$ & $\begin{array}{c}\text { Frecuencia de } \\
\text { monitoreo }\end{array}$ & Respuesta clínica \\
\hline 0 & Mínimo cada 8 horas & $\begin{array}{l}\text { Mantiene controles de enfermería } \\
\text { habituales }\end{array}$ \\
\hline $1-4$ & Mínimo cada 4-6 horas & $\begin{array}{l}\text { Score 1-2: cada } 6 \text { horas } \\
\text { Score } 3-4 \text { : cada } 4 \text { horas }\end{array}$ \\
\hline $\begin{array}{c}5 \text { o más } \\
\text { o } \\
3 \text { puntos } \\
\text { en algún } \\
\text { parámetro }\end{array}$ & Mínimo cada hora & $\begin{array}{l}\text { Evaluación por médico } \\
\text { residente de turno en } \\
\text { máximo } 10 \text { minutos. } \\
\text { - Definir la frecuencia de los } \\
\text { controles posterior a la } \\
\text { evaluación. } \\
\text { - Comunicar del caso a médico } \\
\text { tratante. } \\
\text { - Potencial traslado a UCI, de } \\
\text { persistir deterioro. }\end{array}$ \\
\hline
\end{tabular}




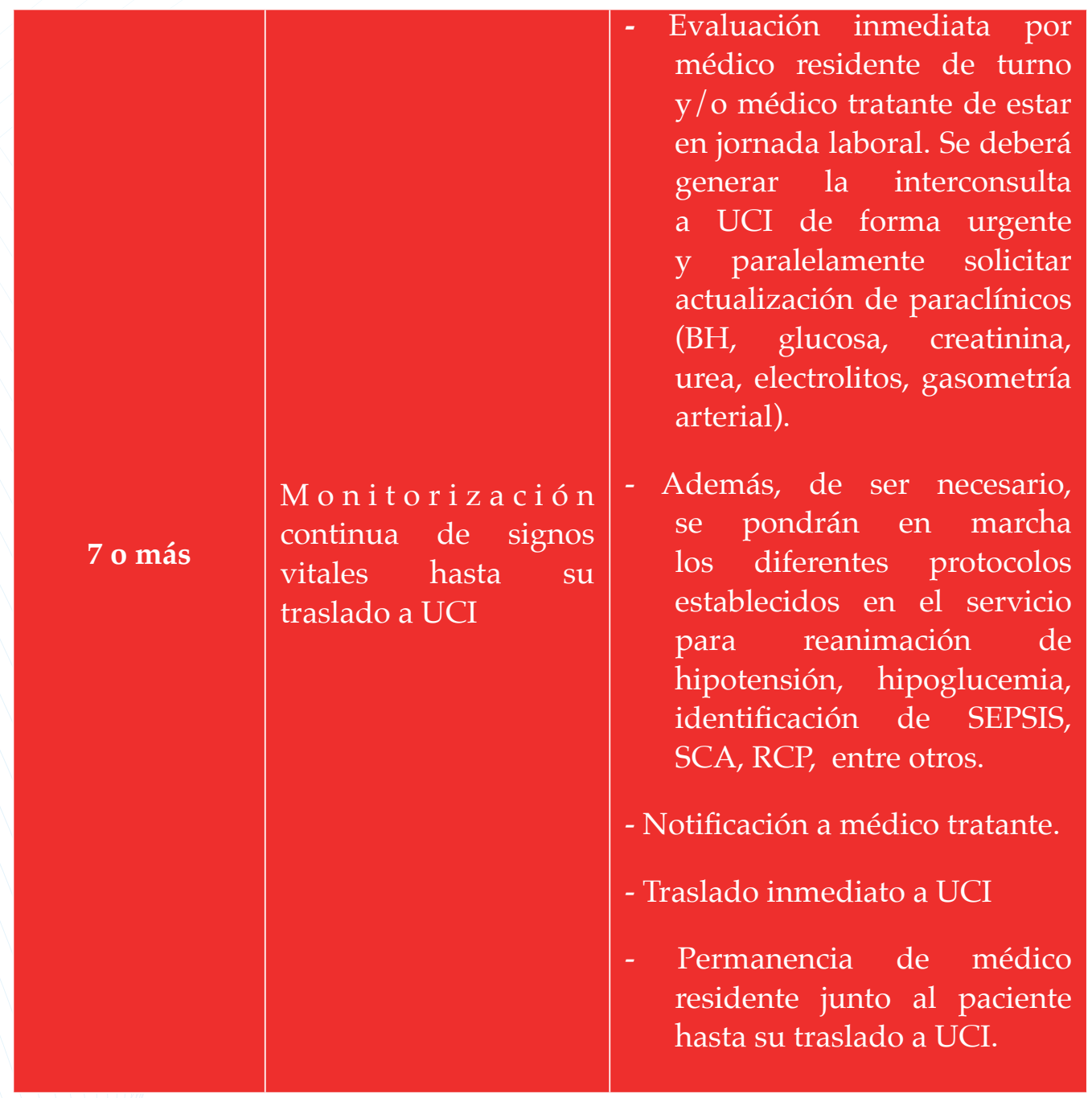

\begin{tabular}{|c|c|}
\hline \multicolumn{2}{|l|}{ ESCALA q-SOFA } \\
\hline Parámetro & Valor \\
\hline Frecuencia respiratoria & $\geq 22$ respiraciones por minuto \\
\hline Presión arterial sistólica & $\leq \mathrm{a} 100 \mathrm{mmHg}$ \\
\hline Escala coma de Glasgow & $\leq \mathrm{a} 13$ puntos \\
\hline \multicolumn{2}{|c|}{$\begin{array}{l}\text { ** Con un valor mayor o igual a dos parámetros, la escala es positiva. Alta } \\
\text { sospecha de SEPSIS, en el contexto clínico del paciente. Calcule la escala de } \\
\text { SOFA completa. }\end{array}$} \\
\hline \multicolumn{2}{|l|}{ ESCALA SIRS } \\
\hline Parámetro & Valor \\
\hline Leucocitos & $>12000 /$ cc o $<4000 /$ cc, o $>10 \%$ bandas \\
\hline
\end{tabular}




\begin{tabular}{|l|l|}
\hline Temperatura & $>38^{\circ} \mathrm{o}<36^{\circ}$ \\
\hline Frecuencia cardíaca & $>90$ latidos por minuto \\
\hline Frecuencia respiratoria & $\begin{array}{l}>20 \text { respiraciones por minuto o } \mathrm{PaCO} 2<32 \\
\mathrm{mmHg}\end{array}$ \\
\hline ** Con un valor mayor o igual a dos parámetros, la escala es positiva. Alta \\
sospecha de SEPSIS, en el contexto clínico del paciente.
\end{tabular}

\section{Versión adaptada para la Pandemia de Covid-19:}

\begin{tabular}{|c|c|c|}
\hline $\begin{array}{c}\text { PUNTAJE } \\
\text { NEWS } 2 \\
\text { MODIFICADO }\end{array}$ & Aislamiento & Intervención \\
\hline $0-4$ & Domicilio & $\begin{array}{l}\text { Se colocará al paciente y a su } \\
\text { acompañante (si es el caso) mascarilla } \\
\text { quirúrgica y gel antiséptico en manos. } \\
\text { El paciente será ubicado temporalmente } \\
\text { en una área aislada anexa mientras se } \\
\text { efectiviza el traslado a domicilio. } \\
\text { El paciente debe ser trasladado en } \\
\text { ambulancia hasta su domicilio en } \\
\text { compañía de personal de salud. Deberá } \\
\text { existir un registro o respaldo de ésta } \\
\text { intervención. } \\
\text { Consejería sobre las medidas de } \\
\text { aislamiento en el domicilio y manejo de } \\
\text { los potenciales contactos. } \\
\text { Cumplimiento de normas, protocolos } \\
\text { y lineamientos vigentes desde nivel } \\
\text { central, MSP, OMS, OPS. Notificación a } \\
\text { Epidemiología. }\end{array}$ \\
\hline $\begin{array}{c}5 \text { - } 7 \\
\text { ó } \\
3 \text { puntos } \\
\text { en algún } \\
\text { parámetro }\end{array}$ & Hospitalización & $\begin{array}{l}\text { Se colocará al paciente y a su } \\
\text { acompañante (si es el caso) mascarilla } \\
\text { quirúrgica y gel antiséptico en manos }\end{array}$ \\
\hline
\end{tabular}




\begin{tabular}{|c|c|c|}
\hline $\begin{array}{c}5 \text { - } 7 \\
\text { ó } \\
3 \text { puntos } \\
\text { en algún } \\
\text { parámetro }\end{array}$ & Hospitalización & $\begin{array}{l}\text { El paciente será ubicado temporalmente } \\
\text { en una área aislada anexa al triage o a } \\
\text { la emergencia mientras se efectiviza el } \\
\text { traslado a hospitalización. } \\
\text { Se colocará el soporte de oxígeno } \\
\text { necesario si no cumple las metas de } \\
\text { saturación establecidas según la edad y } \\
\text { comorbilidades crónicas. } \\
\text { En elárea deaislamiento anexa temporal, } \\
\text { el paciente será estabilizado, vestido y } \\
\text { colocado las medidas de bioseguridad, } \\
\text { previo a su paso al área de aislamiento } \\
\text { definitiva. } \\
\text { El médico de triage respiratorio o } \\
\text { emergencia, comunicará del caso a } \\
\text { jefe de guardia o médico tratante de } \\
\text { Clínica/UCI, quienes se prepararán } \\
\text { para la recepción del paciente, al igual } \\
\text { que todo el equipo de salud de turno. } \\
\text { Si el paciente fue captado en una Unidad } \\
\text { anexa, deberá ser trasladado a esta casa } \\
\text { de salud en ambulancia, en compañia } \\
\text { de paramédico entrenado para SVA o } \\
\text { médico de la unidad. Garantizando } \\
\text { en todo momento las medidas de } \\
\text { bioseguridad y el adecuado flujo de } \\
\text { comunicación con el jefe de guardia. } \\
\text { Se propenderá en todo momento el } \\
\text { traslado ágil y seguro del paciente a } \\
\text { la unidad de aislamiento definitiva } \\
\text { (Servicio de Clínica). La ruta de acceso } \\
\text { deberá ser corta y sin obstáculos, } \\
\text { previamente establecida. Posterior } \\
\text { al paso del paciente, esta vía será } \\
\text { desinfectada por el personal de limpieza } \\
\text { de forma inmediata. Evitar de forma } \\
\text { estricta el contacto con otras superficies } \\
\text { durante el traslado. }\end{array}$ \\
\hline
\end{tabular}




\begin{tabular}{|c|c|c|}
\hline $\begin{array}{c}5 \text { - } 7 \\
\text { ó } \\
3 \text { puntos } \\
\text { en algún } \\
\text { parámetro }\end{array}$ & Hospitalización & $\begin{array}{l}\text { Una vez ingresado el paciente a la } \\
\text { Unidad de Clínica, se aplicarán los } \\
\text { protocolos de diagnóstico y terapéuticos } \\
\text { correspondientes. Notificación } \\
\text { epidemiológica. La Escala de NEWS } \\
2 \text { modificada deberá ser evaluada y } \\
\text { registrada en la historia clínica cada } 4 \\
\text { horas hasta alcanzar un puntaje inferior } \\
\text { a } 4 \text {, donde luego los controles pasarán a } \\
\text { ser cada } 6 \text { a } 8 \text { horas. } \\
\text { Si en algún control, el puntaje NEWS } 2 \\
\text { modificado, aumenta a un valor mayor } \\
\text { de } 7 \text {, se deberá solicitar valoración breve } \\
\text { por UCI, a la vez que se actualizarán } \\
\text { paraclínicos de laboratorio. Prepararse } \\
\text { para un potencial traslado a UCI, de } \\
\text { persistir el deterioro clínico. }\end{array}$ \\
\hline$>7$ & $\begin{array}{l}\text { Unidad de } \\
\text { Críticos } \\
\text { o Terapia } \\
\text { Intensiva }\end{array}$ & $\begin{array}{l}\text { - Evaluación inmediata al arribo del } \\
\text { paciente por el médico de emergencia } \\
\text { (si es referido de unidad anexa o del } \\
\text { triage respiratorio), o médico residente } \\
\text { o tratante de turno en hospitalización (si } \\
\text { ya se encuentra ingresado en piso). } \\
\text { - Estabilizar al paciente de forma } \\
\text { inmediata, estricta vigilancia } \\
\text { respiratoria, hemodinámica } \\
\text { metabólica. } \\
\text { - Monitorización continua de signos } \\
\text { vitales y registro en historia clínica de } \\
\text { puntaje NEWS } 2 \text { modificado cada hora. } \\
\text { - Se deberá generar la interconsulta a } \\
\text { UCI de forma urgente y paralelamente } \\
\text { solicitar paraclínicos (BH, glucosa, } \\
\text { creatinina, urea, electrolitos, gasometría } \\
\text { arterial, enzimas hepáticas, tiempos de } \\
\text { coagulación, procalcitonina y otros a } \\
\text { criterio del médico que asiste el código } \\
\text { rojo). }\end{array}$ \\
\hline
\end{tabular}


- Además, de ser necesario, se pondrán en marcha los diferentes protocolos establecidos en el Servicio para reanimación de hipotensión, insuficiencia respiratoria aguda, hipoglucemia, identificación de SEPSIS, SCA, RCP, entre otros.

- Preparar al equipo para el traslado inmediato a UCI, previa valoración por el responsable de dicha unidad.

- Permanencia de médico residente o tratante junto al paciente hasta su traslado a UCI, con el EPP completo, con los insumos, equipos y medicamentos requeridos para enfrentar un potencial paro cardiorespiratorio durante la

Unidad de Críticos

o Terapia Intensiva movilización.

- Se propenderá en todo momento el traslado ágil y seguro del paciente a la unidad de cuidados intensivos. La ruta de acceso deberá ser corta y sin obstáculos, previamente establecida. Posterior al paso del paciente, esta vía será desinfectada por el personal de limpieza de forma inmediata. Evitar de forma estricta el contacto con otras superficies durante el traslado.

- Una vez que el paciente se encuentre en Cuidados Intensivos, se adoptarán los diferentes protocolos diagnósticos y terapéuticos propios de la Unidad. Notificación a Epidemiología. 
Nota: Evite en lo posible el traslado innecesario del paciente sospechoso, probable o confirmado de Covid-19 a otras dependencias $u$ otras Unidades de Salud, para la realización de procedimientos diagnósticos. Solo considerarlo en el caso de que el BENEFICIO supere al RIESGO de propagación del virus.

\section{Bibliografía}

1. Royal College of Physicians. National Early Warning Score (NEWS 2): Standardising the assessment of acute- illness severity in the NHS. Report of a working party. London: RCP, 2017. - Singer M, Deutschman CS, Seymour CW, et al.

2. The Third International Consensus Definitions for Sepsis and Septic Shock (Sepsis-3). JAMA 2016; 315:801.

3. Levy MM, Fink MP, Marshall JC, et al. 2001 SCCM /ESICM/ACCP/ ATS/SIS International Sepsis Definitions Conference. Crit Care Med 2003; 31:1250. 


\section{APÉNDICE IV.}

\section{PUNTUACIÓN DE ALERTA TEMPRANA PARA COVID-19}

\section{Utilidad}

La nueva puntuación de alerta temprana para COVID-19 fue desarrollada como una herramienta útil para la detección temprana, rápida y precisa de pacientes altamente sospechosos de SARS-CoV-2.

\section{Parámetros}

\begin{tabular}{|l|c|c|}
\hline \multicolumn{1}{|c|}{ CRITERIOS } & CARCATERÍSTICAS & PUNTAJE \\
\hline Signos de Neumonía en TAC & Sí & 5 \\
\hline $\begin{array}{l}\text { Antecedente de contacto cercano } \\
\text { con paciente confirmado con } \\
\text { COVID-19 }\end{array}$ & Sí & 5 \\
\hline Fiebre & Sí & 3 \\
\hline Edad & Maculino años & 1 \\
\hline Sexo respiratorios & $\geq 37.8^{\circ} \mathrm{C}$ & 1 \\
\hline Temperatura máxima & $\geq 1$ síntoma & 1 \\
\hline $\begin{array}{l}\text { Síntomas } \\
\text { significativos (incluido } \\
\text { expectoración y disnea) }\end{array}$ & $\geq 5.8$ & 1 \\
\hline \begin{tabular}{l} 
INL \\
\hline
\end{tabular} & & 1 \\
\hline
\end{tabular}

Temperatura máxima: la temperatura corporal más alta desde el inicio de la enfermedad hasta el primer ingreso hospitalario

INL: Índice Neutrófilo/Linfocito

TAC: Tomografía Axial Computarizada 


\section{Interpretación}

\begin{tabular}{|c|c|}
\hline PUNTUACIÓN & INTERPRETACIÓN \\
\hline $\mathbf{2 1 0}$ & Paciente altamente sospechoso de COVID-19 \\
\hline
\end{tabular}

\section{Bibliografía}

\section{Original/Primaria}

Cong-Ying Song, Jia Xu, Jian-Qin H, Yuan-Qiang Lu. 'COVID-19 puntuación de alerta temprana: una herramienta de detección de parámetros múltiples para identificar pacientes altamente sospechosos. MedRxiv [Internet]. 2020 [citado marzo 2020]. Disponible en: https: / / www.medrxiv. org/content/10.1101/2020.03.05.20031906v1.

\section{Validación}

Li Q, Guan X, Wu P, et al. Dinámica de transmisión temprana en Wuhan, China, de neumonía infectada por coronavirus. N Engl J Med [Internet]. 2020 [citado marzo 2020]. Disponible en: https: / /www.nejm.org/doi/ full/10.1056/ NEJMoa2001316.

Rothe C, Schunk M, Sothmann P, et al. Transmisión de la infección 2019nCoV desde un contacto asintomático en Alemania. N Engl J Med [Internet]. 2020 [citado marzo 2020]. Disponible en: https://www.nejm.org/ doi / full/10.1056/ NEJMc2001468

Phan LT, Nguyen TV, Luong QC, et al Importación y transmisión de humano a humano de un nuevo coronavirus en Vietnam. N Engl J Med [Internet]. 2020 [citado marzo 2020];382:872-4. Disponible en: https: / / www. ncbi.nlm.nih.gov/pubmed/31991079

Holshue ML, DeBolt C, Lindquist S, et al. Primer caso del nuevo coronavirus 2019 en los Estados Unidos. N Engl J Med [Internet]. 2020 [citado marzo 2020]; 382: 929-936. Disponible en: https: / /www.nejm.org/doi/ full/10.1056/NEJMoa2001191 


\section{APÉNDICE V.}

\section{FÓRMULAS MEDICINA BASADA EN EVIDENCIA}

\begin{tabular}{|c|c|c|}
\hline & $\begin{array}{c}\text { CARACTERÍSTICA } \\
\text { EVALUADA } \\
\text { PRESENTE } \\
\text { (prueba de referencia }+ \text { ) }\end{array}$ & $\begin{array}{c}\text { CARACTERÍSTICA } \\
\text { EVALUADA } \\
\text { AUSENTE } \\
\text { (prueba de referencia -) }\end{array}$ \\
\hline $\begin{array}{c}\text { PRUEBA } \\
\text { DIAGNÓSTICA (+) O } \\
\text { EXPOSICIÓN }\end{array}$ & Verdaderos positivos (VP) & Falsos positivos (FP) \\
\hline PRESENTE & $\mathrm{a}$ & $\mathrm{b}$ \\
\hline $\begin{array}{c}\text { PRUEBA } \\
\text { DIAGNÓSTICA (-) O } \\
\text { EXPOSICIÓN }\end{array}$ & Falsos negativos (FN) & $\begin{array}{l}\text { Verdaderos negativos } \\
\text { (VN) }\end{array}$ \\
\hline AUSENTE & c & d \\
\hline
\end{tabular}

\section{A) Pruebas diagnósticas}

Sensibilidad

$$
\frac{\mathrm{a}}{\mathrm{a}+\mathrm{c}}
$$

Especificidad

$$
\frac{d}{d+b}
$$


Valor predictivo positivo

$\frac{a}{a+b}$

Valor predictivo negativo

$\frac{d}{d+c}$

Prevalencia

$$
\frac{a+c}{a+b+c+d}
$$

Exactitud

$$
\frac{a+d}{a+b+c+d}
$$

Razón de verosimilitud positivo

$$
\begin{gathered}
\frac{\left(a^{*}(b+d)\right)}{\left(b^{*}(a+c)\right)} \\
\text { ó } \\
\frac{\text { Sensibilidad }}{\text { (1-Especificidad })}
\end{gathered}
$$

Razón de verosimilitud negativo

$$
\begin{gathered}
\frac{\left(\mathrm{a}^{*}(\mathrm{~b}+\mathrm{d})\right)}{\left(\mathrm{b}^{*}(\mathrm{a}+\mathrm{c})\right)} \\
\text { ó } \\
\text { 1-Sensibilidad } \\
\text { Especificidad }
\end{gathered}
$$

Probabilidad pre prueba = prevalencia de un evento reportado en la literatura u obtenida en estudio de investigación previo. 
Momio pre prueba

$$
\frac{\text { Prevalencia }}{\text { (1-prevalencia) }}
$$

Momio pos prueba

(Momio pre prueba) * $(\mathrm{RV}$ positivo)

Probabilidad pos prueba

$$
\frac{\text { Momio pos prueba }}{\text { (Momio pos prueba+1) }}
$$

\section{B) Estudios prospectivos o de cohortes}

Incidencia en expuestos $=(\mathrm{a} / \mathrm{a}+\mathrm{b})$

Incidencia en no expuestos $=(\mathrm{c} / \mathrm{c}+\mathrm{d})$

Riesgo relativo $(R R)=($ incidencia expuestos/incidencia no expuestos $)$

Riesgo absoluto (RA) = (incidencia expuestos / incidencia no expuestos)

\section{C) Estudios retrospectivos o casos y controles}

Momio de $\operatorname{casos}=(\mathrm{a} / \mathrm{c})$

Momio de no casos $=(\mathrm{b} / \mathrm{d})$

Razón de momios = momio de casos $/$ momio no casos

\section{D) Ensayos Clínicos Controlados}

Incidencia en experimental $=(a / a+b)$

Incidencia en No experimental $=(\mathrm{c} / \mathrm{c}+\mathrm{d})$

Riesgo relativo $(R R)=$ (Incidencia experimental/incidencia No experimental) 


\section{E) Estudios transversales}

Prevalencia en expuestos $=(a / a+b)$

Prevalencia en No expuestos $=(\mathrm{c} / \mathrm{c}+\mathrm{d})$

Razón de prevalencia $(\mathrm{RP})=($ Prevalencia en expuestos / prevalencia No expuestos)

\section{F) Chi cuadrado}

$\frac{\left(\left(a^{*} d\right)-\left(b^{*} c\right)^{2} N\right)}{\left(m 1^{*} m 0^{*} n 1^{*} n 0\right)}$

\section{G) Intervalo de confianza}

$\operatorname{RR}^{1 \pm(1.96+\sqrt{ } \mathrm{c} \text { hi2 })}$

H) Tamaño de muestra y Riesgo Relativo

$\mathrm{n}=\mathrm{Za}^{2}$ * $\left(((\mathrm{q} 1 / \mathrm{p} 1)+(\mathrm{q} 2 / \mathrm{p} 2)) /(\ln (1-\mathrm{E}))^{2}\right)$

$\mathrm{RR}=(\mathrm{p} 1 / \mathrm{p} 2)$

Ensayos Clínicos Controlados Aleatorizados

$\mathrm{NNT}=1 / \mathrm{RA}$

$\mathrm{RA}=($ incidencia experimental - incidencia No experimental)

J) NNT e Intervalo de Confianza

$\mathrm{a} \% \mathrm{IC}=(\mathrm{p} 1-\mathrm{p} 2) \pm \mathrm{Z} \alpha \sqrt{ }((\mathrm{p} 1 \mathrm{q} 1) / \mathrm{n} 1)+(\mathrm{p} 2 \mathrm{q} 2 / \mathrm{n} 2))$ 
"El conocimiento al servicio de la comunidad" 


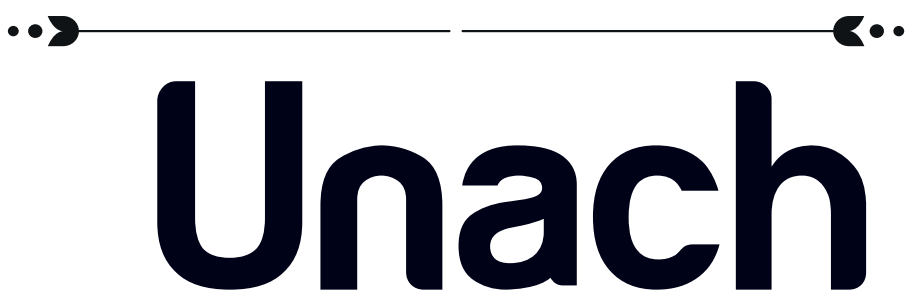

UNIVERSIDAD NACIONAL DE CHIMBORAZO

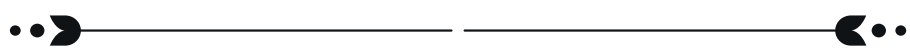

Gestión del Conocimiento y Propiedad Intelectual

Cálculos y Escalas pronósticas en Medicina Interna; se publicó en el mes de mayo de 2020 en la Universidad Nacional de Chimborazo. 


\section{CÁlCULOS $Y$ ESCALAS PRONÓSTICAS EN MEDICINA INTERNA}

Luego del lanzamiento de la obra titulada "Protocolos Terapéuticos en Medicina Interna", donde su contenido bibliográfico permitió un acercamiento a la información científica más relevante y actualizada en el campo clínico, surgió la necesidad de buscar un complemento que identifique los principales cálculos y escalas pronósticas, utilizadas frecuentemente en cada una de las subespecialidades de la medicina interna. Somos conscientes que el análisis clínico representa un proceso complejo, desde la recopilación de la información a través de la historia clínica, hasta el análisis exegético basado en problemas, con la finalidad de identificar la mejor conducta diagnóstica y terapéutica; sin embargo, en este camino es imprescindible hacer uso de escalas, fórmulas y demás cálculos validados por estudios científicos de alto impacto, para analizar probabilidades, predecir complicaciones, riesgos o simplemente evaluar la respuesta a un tratamiento determinado. Todas éstas intervenciones, nos permitirán tomar decisiones de forma oportuna, y por ende mejorar la sobrevida de nuestros pacientes. "Cálculos y escalas pronósticas en Medicina Interna", bajo ningún concepto es una herramienta aislada en la práctica clínica diaria, requiere del juicio y criterio de cada profesional o especialista en su área para la aplicación eficiente en cada paciente.

Héctor Ortega C. MD, MPH, PhD(c)
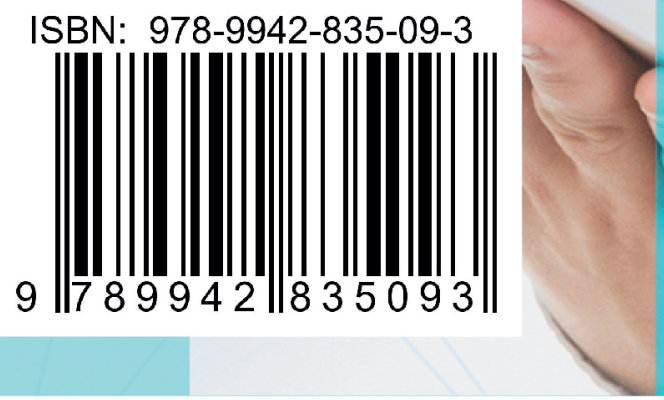

ISBN: 978-9942-835-10-9

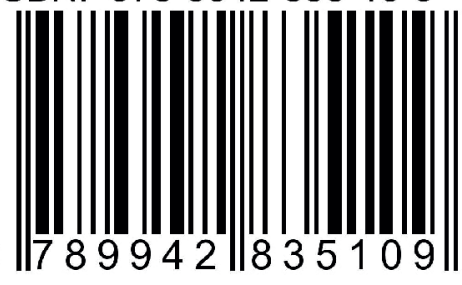

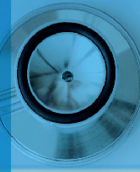
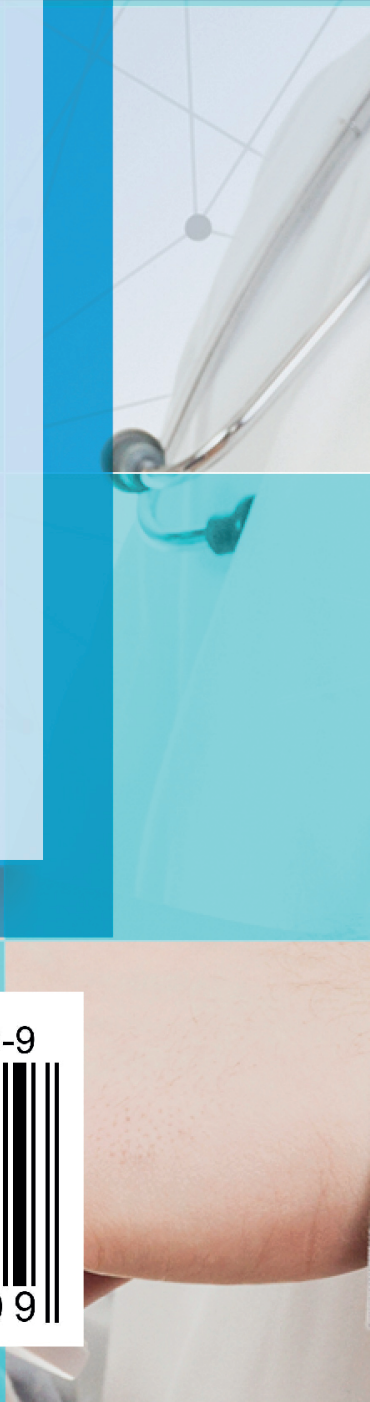

\section{Unach \\ VICERRECTORADO DE INVESTIGACIÓN, VINCULACIÓN Y POSGRADO \\ DIRECCIÓN DE INVESTIGACIÓN \\ GESTIÓN DEL CONOCIMIENTO
YPROPIEDAD INTELECTUAL}

